\title{
Wirkung im Coaching
}

Herausgegeben von Robert Wegener/Silvia Deplazes / Marianne Hänseler/Hansjörg Künzli/ Stefanie Neumann / Annamarie Ryter/ 


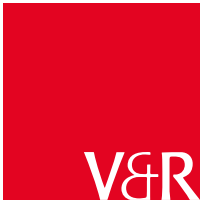

(c) 2018, Vandenhoeck \& Ruprecht $\mathrm{GmbH} \&$ Co. KG, Göttingen https://doi.org/10.13109/9783666402975 | CC BY-NC-ND 4.0 
(c) 2018, Vandenhoeck \& Ruprecht $\mathrm{GmbH} \&$ Co. KG, Göttingen https://doi.org/10.13109/9783666402975 | CC BY-NC-ND 4.0 
Robert Wegener/Silvia Deplazes/Marianne Hänseler/ Hansjörg Künzli/Stefanie Neumann/Annamarie Ryter/ Wolfgang Widulle (Hg.)

\section{Wirkung im Coaching}

Mit 31 Abbildungen und 10 Tabellen

Vandenhoeck \& Ruprecht 
Bibliografische Information der Deutschen Nationalbibliothek:

Die Deutsche Nationalbibliothek verzeichnet diese Publikation in der Deutschen Nationalbibliografie; detaillierte bibliografische Daten sind im Internet über https://dnb.de abrufbar.

(C) 2018, Vandenhoeck \& Ruprecht GmbH \& Co. KG, Theaterstraße 13, D-37073 Göttingen Das Werk und seine Teile sind urheberrechtlich geschützt.

Jede Verwertung in anderen als den gesetzlich zugelassenen Fällen bedarf der vorherigen schriftlichen Einwilligung des Verlages.

Umschlagabbildung: ILeysen/shutterstock.com

Satz: SchwabScantechnik, Göttingen

Vandenhoeck \& Ruprecht Verlage | www.vandenhoeck-ruprecht-verlage.com

ISBN (Print): 978-3-525-40297-9

ISBN (PDF): 978-3-666-40297-5

https://doi.org/10.13109/9783666402975

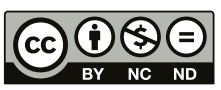

Dieses Material steht unter der Creative-Commons-Lizenz Namensnennung - Nicht kommerziell - Keine Bearbeitungen 4.0 International. Um eine Kopie dieser Lizenz zu sehen, besuchen Sie http://creativecommons.org/licenses/by-nc-nd/4.0/. 


\section{Inhalt}

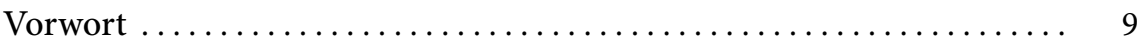

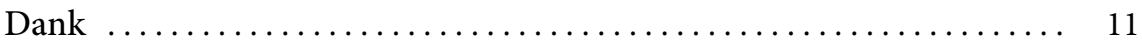

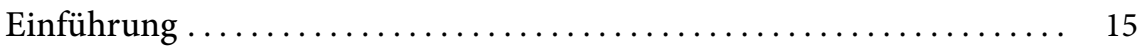

Über die Notwendigkeit institutionell verankerter

Coaching-Forschung

Robert Wegener, Silvia Deplazes, Marianne Hänseler, Hansjörg Künzli,

Stefanie Neumann, Annamarie Ryter und Wolfgang Widulle

\section{Teil 1 - Wissenschaftliche Fachbeiträge}

Peter oder Petra?

Die Rolle von Geschlecht und Führungsqualitäten in der Bewertung und Auswahl von Coaches

Maximilian D. Mühlberger und Eva Traut-Mattausch

Wo führt das hin?

Effekte des Coaching-Führungsstils auf das Erleben der Klientinnen und Klienten und den Coaching-Erfolg Sabine Losch und Eva Traut-Mattausch

Die Bedeutung von Medien für die Qualität von Coaching .......... 46 Harald Geißler

Coaching und die Rolle des Unbewussten

Neurowissenschaftliche Erkenntnisse für eine wirksame Coaching-Praxis Alica Ryba

Das Selbst im Bild

Mit Bildern Selbstreflexion und Veränderung im Coaching wirksam unterstützen Jasmin Messerschmidt 
Prozessbezogene Determinanten der Wirkung von Einzelcoaching .....

Ein systematischer Überblick über den internationalen Forschungsstand Cornelia Tonhäuser

»Turning Duty into Joy!

Optimierung der Selbstregulation im Coaching durch Motto-Ziele Julia Weber

Mein Coaching!

Die Erfüllung des Bedürfnisses nach Autonomie

Sandra J. Schiemann, Christina Mühlberger, Maximilian D. Mühlberger,

Isabell Braumandl und Eva Jonas

Transferstärke-Coaching . .

Selbstlernkompetenz fördern und Lerntransfer sichern

Axel Koch

Ambivalenzen des Coachings

Über nicht intendierte Wirkungen in Zeiten der Selbstoptimierung

Laura Glauser

Das Impostor-Phänomen - ein Thema im Coaching?

Eva Traut-Mattausch und Mirjam Zanchetta

Effectiveness of Leadership Coaching 148

Willem Jan Hofmans

\section{Teil 2 - Forschungsrelevante Beiträge aus der Praxis}

Formatkompetenz von Coaches

Design von maßgeschneiderten Vorgehensweisen im Coaching

Elke Berninger-Schäfer und Efriom Kineselassie

»Blended Business Coaching « mit dem Laufbahnkoffer HR

Neue Formen des Coachings in der beruflichen Weiterbildung

Wolfgang Eberling, Gery Bruederlin und Marion Alt

Kein Coaching ohne Evaluation der Ergebnisse

Niki Harramach und Nina Veličković 
Führung entwickeln und Wirkung entfalten

Zum Zusammenhang von Coaching und Change-Management Mathias Hofmann

Coaching-Kultur in deutschsprachigen Organisationen

Das Konzept der resilienten und agilen Organisation

Axel Klimek und Werner Stork

Die Autorinnen und Autoren, Herausgeberinnen und Herausgeber . .

221 
(c) 2018, Vandenhoeck \& Ruprecht $\mathrm{GmbH} \&$ Co. KG, Göttingen https://doi.org/10.13109/9783666402975 | CC BY-NC-ND 4.0 


\section{Vorwort}

Globalisierung und Digitalisierung fordern Anpassungs- und Veränderungsprozesse in vielen Lebens-, Produktions- und Dienstleistungsbereichen. Im Spannungsfeld von Informationsflut und Spezialisierungsdruck wird eine effiziente Wissensaneignung und Entscheidungsfindung für den Einzelnen immer schwieriger. Erhöhte und teils widersprüchliche Anforderungen der Arbeitswelt sowie familiäre Ansprüche erfordern flexible Bewältigungsstrategien. In Folge dieser Entwicklung steigt die Nachfrage an professionellen Problemlösungskompetenzen und -ressourcen, was wiederum in einem rasanten Wachstum einer disziplinenübergreifenden Beratungsbranche zum Ausdruck kommt. Ursprünglich stigmatisiert als »Hilfsmaßnahme für schlechte Managementleistung « und unterschätzt als kurzfristiger Modetrend, ist Coaching als Beratungsformat inzwischen für verschiedene Themen, Settings und Zielgruppen zum Mittel der Wahl avanciert. Statt Standardlösungen sind individualisierte Entwicklungsfortschritte, an den Bedürfnissen des Klienten oder der Klientin orientiert, gefragt. Coaches unterstützen ihre Klientinnen und Klienten (Coachees) in einem kollaborativen Prozess darin, Ziele zu erarbeiten, Lösungen zu entwickeln und umzusetzen, sich beruflich und persönlich zu entwickeln und ein erhöhtes Maß an Selbstreflexion, Zielerreichung und Wohlbefinden zu erlangen. Die Vielzahl an Coaching-Angeboten, die wachsende Zahl von Coaches und die ebenfalls wachsende Zahl an Aus-, Fort- und Weiterbildungsangeboten in diesem Bereich zeugen von der Popularität des aus der Praxis formierten Formates. Der Coaching-Boom ist ungebrochen und entspricht unserem individualisierten, enttraditionalisierten Menschenbild in der Multioptionsgesellschaft. Nichtsdestotrotz wird der Ruf nach evidenzbasiertem Coaching lauter, um die Ausgaben für Coaching zu legitimieren, Coaching zu verbessern und die Professionalisierung voranzutreiben. Einschlägige empirische Studien sind seit den 1990er Jahren zu finden. Sie beschränken sich jedoch meist auf ergebnisorientierte, quantitativ-methodische Wirksamkeitsevaluationen, häufig ohne Vergleichs- oder Kontrollgruppe durchgeführt. Zudem mangelt 
es den bestehenden Untersuchungen häufig an einer Standardisierung oder einer angemessenen Stichprobengröße. Systematische Betrachtungen des Coaching-Prozesses sind zudem kaum zu finden. Es gibt zwar erste Hinweise, dass Coaching wirkt, aber die Frage, was wie wirkt, ist erst in Ansätzen beantwortet. Praktikerinnen und Praktiker, Berufsverbände, Forscher und Forscherinnen stimmen darin überein, dass die größte Herausforderung des Coachings in seiner Professionalisierung liegt. Professionalisierung kommt ohne Evidenzbasierung nicht aus und kann nur durch einen konstruktiven Dialog zwischen Praktikern und Praktikerinnen, Lehrinstituten, Forscherinnen und Forschern erfolgen.

Die Fachhochschule Nordwestschweiz leistet mit ihren internationalen Coaching-Kongressen einen sehr wertvollen Beitrag, Praxis und Forschung zusammenzubringen, den Dialog zu initiieren und aufrechtzuerhalten - einen Beitrag, der nicht hoch genug eingeschätzt werden kann. Der vorliegende Band unter dem Titel »Wirkung im Coaching«, der aus Beiträgen zum 4. Internationalen Coaching-Kongress zum Thema "Wirkung, Qualität und Evaluation im Coaching « beruht, ist sichtbares Zeugnis dieses Austausches.

Braunschweig, im Herbst 2017

Simone Kauffeld 


\section{Dank}

Der vorliegende Band »Wirkung im Coaching « besteht aus Beiträgen zum 4. Internationalen Coaching-Kongress "Wirkung, Qualität und Evaluation im Coaching «, der am 14. und 15. Juni 2016 in Olten/Schweiz stattfand, organisiert durch die Hochschule für Soziale Arbeit der Fachhochschule Nordwestschweiz FHNW. Für die Bereitschaft, sich an den Anforderungen der Herausgebenden zu orientieren, sei den Autorinnen und Autoren herzlich gedankt. Wir danken ferner den folgenden Partnern für ihre Unterstützung, die den Kongress und diese Publikation erst ermöglicht hat:

\section{Förderorganisationen}

- SGSA Schweizerische Gesellschaft für Soziale Arbeit

- SNF Schweizerischer Nationalfonds zur Förderung der wissenschaftlichen Forschung

\section{Hauptsponsoren}

- BSO Berufsverband für Coaching, Supervision und Organisationsberatung

- DGSv Deutsche Gesellschaft fur Supervision und Coaching e. V.*

- EMCC European Mentoring and Coaching Council Schweiz

- FSP Föderation der Schweizer Psychologinnen und Psychologen

- KICK Institut für Coaching und Kommunikation

- OEVS Österreichische Vereinigung für Supervision und Coaching

- SGfB Schweizerische Gesellschaft für Beratung 


\section{Hochschulpartner}

- Hochschule für Wirtschaft der Fachhochschule Nordwestschweiz FHNW

- Institute of Coaching, Harvard Medical School

- Pädagogische Hochschule der Fachhochschule Nordwestschweiz FHNW

- Universität Salzburg, Universitätslehrgang für Supervision, Coaching und Mediation

- Departement Angewandte Psychologie, Zürcher Hochschule für Angewandte Wissenschaften ZHAW

\section{Unternehmenspartner}

- Coaching Company Pro GmbH

- CZO Coachingzentrum Olten

- CoBeCe Coaching- \& Beratungs-Centrum

- C for C Coaching for Core

- Change Coaching GmbH

- Curaviva Weiterbildung

- isi institut für systemische impulse

- SHS CONSULT

- Tobler Management

- Trigon Entwicklungsberatung

- Wilob Weiterbildungsinstitut für lösungsorientierte Therapie und Beratung

\section{Verbandspartner}

- ACC Austrian Coaching Council

- ANSE Association of National Organisations for Supervision in Europe

- DBVC Deutscher Bundesverband Coaching e. V.*

- DCV Deutscher Coaching Verband e. V.*

- DGfC Deutsche Gesellschaft für Coaching e. V.*

- DGSF Deutsche Gesellschaft für Systemische Therapie, Beratung und Familientherapie e. V.*

- EASC European Association for Supervision and Coaching e. V. ${ }^{*}$

- ECA European Coaching Association

- EMCC European Mentoring \& Coaching Council Deutschland e. V.*

- ICF International Coach Federation Switzerland 
- Roundtable der Coachingverbände

- SCA Swiss Coaching Association

- SSCP Swiss Society for Coaching Psychology

- UBIT Fachgruppe Wien Unternehmensberatung, Buchhaltung und Informationstechnologie

\section{Verlags- und Medienpartner}

- BILDUNGaktuell

- Coaching Magazin

- HR Today

- Lehmanns Media

- Organisationsberatung, Supervision, Coaching (OSC)

- Persorama/HR Swiss

- Springer VS

Ein großer Dank geht an Cheryl Meyer, die als unterstützende Fachkraft zur Entstehung dieses Buches maßgeblich beigetragen hat. Ein herzlicher Dank geht auch an Christoph Gassmann, der das Manuskript mit kritischem Blick, inhaltlichem Flair und großer Sorgfalt lektoriert und korrigiert hat. Nicht zuletzt danken wir dem Vandenhoeck \& Ruprecht Verlag, und insbesondere Imke Heuer, für die kompetente Begleitung. 
(c) 2018, Vandenhoeck \& Ruprecht $\mathrm{GmbH} \&$ Co. KG, Göttingen https://doi.org/10.13109/9783666402975 | CC BY-NC-ND 4.0 


\section{Einführung}

\section{Über die Notwendigkeit institutionell verankerter Coaching-Forschung}

Robert Wegener, Silvia Deplazes, Marianne Hänseler, Hansjörg Künzli,
Stefanie Neumann, Annamarie Ryter und Wolfgang Widulle

Coaching ist eine personenorientierte Form der Beratung, die zunehmend Anerkennung findet. Das übergeordnete Ziel von Coaching besteht darin, private und berufliche Leistungs- und Handlungsziele zu identifizieren und $\mathrm{zu}$ erreichen (Loebbert, 2017; Wegener, 2017). Auf der Basis von (Selbst-)Reflexion und orientiert an den Ressourcen und Stärken der Coachees werden zielführende Entwicklungs- und Veränderungsprozesse initiiert und professionell begleitet. Als übergeordnete Problemstellung, die mit Coaching adressiert wird (Fietze, 2011), geht es immer auch darum, individuelle Selbststeuerung im Kontext von rapide sich verändernden und komplexer werdenden Gesellschaften zu verbessern (Fietze, 2016; Greif, 2014; Stelter, 2014). Vor diesem Hintergrund hält Coaching heute Einzug in unterschiedliche gesellschaftliche Handlungsfelder (Wegener et al., 2015; Wegener, Loebbert \& Fritze, 2015; Wegener, Loebbert \& Fritze, 2016a; Wegener, Loebbert \& Fritze, 2016b). Die Etablierung sogenannter »Coaching-Praxisfelder « (z. B. Business-Coaching, Coaching in der Arbeitsintegration oder Coaching zur Förderung von Gesundheit) ist im Sinne von Selbstorganisation auch als individuelle Antwort auf gesellschaftlich bedingte Herausforderungen zu verstehen. Dabei besteht, dies sei nicht verschwiegen, immer Gefahr, dass der Begriff »Coaching « als Etikett allem Möglichen angeheftet wird, dass also der Begriff zunehmend verwässert wird (vgl. z. B. Lippmann, 2007).

Mit der rasanten Verbreitung von Coaching im Zusammenhang steht, dass es sich in einem intensiven Professionalisierungsprozess befindet (Fietze, 2015; Schreyögg, 2013, S. 420). Nach einer zunächst »stürmischen Marktetablierung « in den 1980er Jahren, einer bis heute anhaltenden Angebotsdifferenzierung und einer ständigen Zunahme an Verbänden, ist seit der Jahrtausendwende auch eine signifikante Zunahme an Forschung zu verzeichnen (Birgmeier, 2006; Böning \& Fritschle, 2005; Fietze, 2011). Aus Sicht der Professionssoziologie wird insbesondere Letzteres als starkes Zeichen von Professionalisierung gedeutet (Fietze, 2011). Entwicklung und Ausgang des laufenden Professionalisierungs- 
prozesses können zum jetzigen Zeitpunkt nur schwer eingeschätzt werden. Einige Zeichen deuten darauf hin, dass in der teils unüberschaubaren Angebotsvielfalt die Qualitätsansprüche an Coaching generell steigen. Dies ist aus Sicht der Autorenschaft zum Beispiel an der Zunahme von Hochschulen zu erkennen, die akademisch fundierte Coaching-Weiterbildungen anbieten, nicht selten kombiniert mit weiteren personenorientierten und organisationsbezogenen Beratungsformaten (Mediation, Supervision, Organisationsberatung). Ähnlich kann auch die Bewegung von Verbänden hin zu Hochschulen gedeutet werden, ob zu Forschungszwecken oder zur Anerkennung akademisch fundierter Weiterbildungen. Ebenso scheint sich die Wissenschaft mehr und mehr für Coaching zu interessieren. An diversen Hochschulen und Lehrstühlen im deutschsprachigen Raum ist Coaching bereits fester Forschungsgegenstand (vgl. z. B. TU Braunschweig, Prof. Dr. Simone Kauffeld; Universität Kassel, Prof. Dr. Heidi Möller; Universität Salzburg, Prof. Dr. Eva Traut-Mattausch und Prof. Dr. Eva Jonas; Zürcher Hochschule für Angewandte Wissenschaften, Prof. Hansjörg Künzli und Prof. Dr. Silvia Deplazes; Hochschule Berlin, Prof. Dr. Carsten Schermuly). Aber Akademisierung alleine reicht nicht. Die Anforderungen an eine Qualifizierung zum Coach werden immer noch kontrovers diskutiert (Lippmann, 2015).

Ein präziser Blick auf den Stand der Forschung (Geißler, 2016; Greif, 2017; Möller \& Kotte, 2011; Myers, 2014; Wegener, 2017) zeigt, das der Akzent bisher vorwiegend bei der ergebnisorientierten Coaching-Forschung lag. Damit wird eine Form der Forschung bezeichnet, die sich mit der Frage beschäftigt, inwiefern Coaching (überhaupt) Wirkung zeigt. Professionssoziologisch erscheint diese Fokussierung im Sinne vor allem der »Legitimationsfunktion« von Forschung bedeutsam; es gilt, die Glaubwürdigkeit von Coaching (auch) wissenschaftlich herzustellen (Fietze, 2011) und so weitere Forschungsabsichten auf ein solides Fundament zu heben. Als Zwischenbilanz zur Frage nach der Wirksamkeit von Coaching sei auf Künzli verwiesen, der in einer Übersicht feststellt, dass Coaching wirkt (Künzli, 2009) - ein Befund, der auch in Metaanalysen neueren Datums (De Meuse, Dai \& Lee, 2009; Jones, Woods \& Guillaume, 2015; Sonesh et al., 2015; Theeboom, Beersmaa \& van Vianena, 2013) beschrieben wird. De Haan und Duckworth (2012) stellen mit Bezug auf »harte« RCT-Studien fest, dass Coaching zwar wirkt, im Vergleich zu (wirksamen) Psychotherapien jedoch weniger stark. Greif (2016), der sich auf diese Erkenntnis bezieht, zeigt sich überzeugt, dass die Wirkung von Coaching durch die Entwicklung entsprechender Coaching-Methoden noch gesteigert werden kann.

Ein zweiter, derzeit noch weniger etablierter Forschungsstrang beschäftigt sich mit der Frage, was in Coaching-Prozessen passiert und welche Faktoren und Bedingungen für den Erfolg von Coaching verantwortlich sind. Diese prozess- 
orientierte Coaching-Forschung ist im Vergleich noch nicht so weit fortgeschritten, aber nicht weniger bedeutend (Deplazes, 2015; Möller \& Kotte, 2011; Wegener, 2017). Ein Vergleich mit der heute gut etablierten Psychotherapie-Forschung (vgl. z. B. Duncan, Miller, Wampold \& Hubble, 2010; Elliott, 2010; Timulak, 2008) macht deutlich, dass die Coaching-Forschung zwar insgesamt ein gutes Stück vorangekommen ist, jedoch nach wie vor als bescheiden bewertet werden muss (Wegener, 2017).

Dass der Stand der Coaching-Forschung noch nicht sonderlich fortgeschritten ist, hat verschiedene Gründe. Prinzipiell ist davon auszugehen, dass es generell Zeit braucht, bis ein Forschungsgegenstand sich zum anerkannten Forschungsfeld entwickelt und etabliert. Bei einem Gegenstand wie Coaching, der nicht eindeutig einer wissenschaftlichen Disziplin zugeordnet werden kann, scheint dieser Befund besonders zuzutreffen. Bei Coaching handelt es sich ja, ähnlich wie bei Gender-Studies oder der Sozialen Arbeit, um einen geradezu prototypisch transdisziplinären Forschungsgegenstand, der Bezüge zu unterschiedlichen akademischen Disziplinen aufweist, etwa zur Psychologie, zu den Erziehungswissenschaften oder der Betriebswirtschaftslehre. Aber auch die Philosophie, die Soziologie, die Linguistik oder die Soziale Arbeit, um nur einige weitere Disziplinen zu erwähnen, stehen in unmittelbarem Zusammenhang mit Coaching als Forschungsgegenstand. Dass es bis heute kaum Coaching-Lehrstühle gibt, macht die Situation nicht einfacher, auch nicht in Bezug auf die Finanzierung entsprechender Forschungsprojekte. Das Fehlen einer programmartigen Coaching-Forschung - wie gefordert (Möller \& Kotte, 2011) hängt ebenfalls mit dem geschilderten Befund zusammen. Entsprechend basiert die heutige Coaching-Forschung mehrheitlich auf punktuellen Einzelstudien und Qualifikationsarbeiten (Dissertationen, M.A./B.A.), die an verschiedenen Fakultäten verfasst werden. Eine stärkere Institutionalisierung und Sichtbarkeit der Coaching-Forschung ist daher neben einer spürbaren Verstärkung an Universitäten und Hochschulen auch auf die Einbindung in eine übergreifende beratungswissenschaftliche Perspektive angewiesen (Schiersmann \& Thiel, 2012). Die noch junge Professionsgeschichte von Coaching braucht die Unterstützung eines internationalen, beratungswissenschaftlich relevanten Netzwerkes (Seel, 2014), aus dem heraus die Coaching-Forschung aktiv vorangetrieben wird, ohne den Blick für die Coaching-Praxis zu verlieren (Fietze, 2017).

Das vorliegende Buch ist im beschriebenen Professionalisierungsprozess von Coaching zu verorten. Aktuelle und relevante Forschungsbeiträge werden vorgestellt und der Forschungs- und Praxis-Community zugänglich gemacht, womit ein Beitrag zum wissenschaftlichen Diskurs von Coaching geleistet wird. Konkret werden Forschungsprojekte präsentiert, die eine inhaltliche Verbindung 
zu dem für Coaching zentralen Begriff der Wirkung aufweisen, ein Thema, das auch am 4. Internationalen Coaching-Kongress "Coaching meets Research" 2016 im Fokus stand. Damit steht die vorliegende Publikation auch im Bezug zu den seit 2010 alle zwei Jahre an der Fachhochschule Nordwestschweiz FHNW ${ }^{1}$ durchgeführten Internationalen Coaching-Kongressen, deren Ziel es ist, die Brücke zwischen Coaching-Praxis und Coaching-Forschung zu stärken.

Wirkung im Coaching ist also der gemeinsame Nenner des Buchs. Der Begriff der Wirkung kann, wie in den einzelnen Beiträgen sichtbar wird, unterschiedliche Vorstellungen transportieren. So kann er sich auf die Wirkweise von Coaching-Prozessen beziehen, also die Art und Weise, wie Wirkung im Verlauf eines Coachings zustande kommt. Thematisiert werden auch Wirkfaktoren - Faktoren, die auf das finale Ergebnis eines Coachings einen Einfluss haben. Weiter geht es in diesem Buch um die Idee von Prozesswirksamkeit und darüber hinaus um die finale Wirksamkeit von Coaching. Es werden ferner Möglichkeiten thematisiert, wie die Wirksamkeit von Coaching durch die Kombination mit anderen Weiterbildungsformaten verbessert werden kann. Last but not least sind Nebenwirkungen im Coaching Thema. Ein Beispiel dafür wäre, wenn die Ursachen für Misserfolge in einem Coaching (z. B. erfolglose Stellensuche) einseitig den beteiligten Individuen und nicht den vorhandenen strukturellen Rahmenbedingungen (z. B. Knappheit von Arbeitsstellen in der Wissenschaft) zugeordnet werden, obwohl diese einen entscheidenden Einfluss haben.

Der Inhalt des vorliegenden Buchs basiert auf Beiträgen, die am 4. Internationalen Coaching-Kongress vorgestellt und zum Zweck dieser Publikation weiterentwickelt und verschriftlicht wurden. Ausgewählt wurden die einzelnen Beiträge entlang sorgfältig definierter Kriterien. Die Struktur der Publikation orientiert sich an der Logik des Coaching-Kongresses, aktuelle Forschungsprojekte und richtungsweisende Entwicklungen der Praxis sichtbar zu machen. So enthält der erste und umfassendere Teil des Buchs Beiträge zu aktuellen Forschungsprojekten. Der zweite, deutlich kürzere Teil der Publikation besteht aus einer Auswahl an praxisorientierten Coaching-Beiträgen.

1 Organisiert werden die Internationalen Coaching-Kongresse vom Institut für Beratung, Coaching und Sozialmanagement der Hochschule für Soziale Arbeit FHNW. Dieses Institut bietet seit rund zwanzig Jahren Coaching-Weiterbildung auf akademischem Niveau an, publiziert und forscht zu Coaching und versteht sich als Plattform für die Entwicklung und Professionalisierung von Coaching (www.coaching-studies.ch). 


\section{Zum Inhalt der Publikation}

\section{Teil 1 - Wissenschaftliche Fachartikel}

Maximilian D. Mühlberger und Eva Traut-Mattausch verfolgen in ihrem Beitrag die Frage, was für Bilder potenzielle Kundinnen und Kunden von Coaches in sich tragen. In einer empirischen Studie - mit der »impliziten Führungstheorie", auf den Kontext von Coaching übertragen, als theoretischem Fundament wurde untersucht, welche geschlechter- und führungsspezifischen Eigenschaften mit erfolgreichen Coaches assoziiert werden und inwiefern diese Eigenschaften die Auswahl eines Coaches beeinflussen.

Sabine Losch und Eva Traut-Mattausch untersuchen auf der Basis von zwei kontrolliert-randomisierten Studien den Einfluss eines transaktional-transformationalen Coaching-Führungsstils auf das Coaching von Studierenden zu Karriereplanung und bezogen auf Aufschiebeverhalten (Prokrastination). Die Autorinnen gelangen zum Schluss, dass die Coaching-Führungsstile Arbeitsbeziehung und Erleben von Autonomie und Motivation stärken und die Coaching-Ergebnisse insgesamt verbessern. Aufgrund der Analyse komplexer Wirkprozesse und -faktoren leistet die Studie einen Beitrag zu einem vertieften Verständnis erfolgreicher Coaching-Prozesse.

Harald Geißler thematisiert die Bedeutung von Medien für die Qualität von Coaching-Prozessen. Er nähert sich dem bis anhin wenig diskutierten Thema anhand einer inhaltlich-konzeptionellen Klärung, was unter Medien verstanden werden kann. Im Anschluss werden mögliche Formen von Coaching-Medien vorgestellt. Eine Reflexion des Zusammenhangs zwischen Qualität und Coaching-Medien erfolgt auf der Grundlage von Plausibilitätseinschätzungen zur Prozesswirksamkeit. Dabei fokussiert Geißler auf das »Virtuelle Coaching «(VC), einen telefon- und internetprogrammbasierten Coaching-Ansatz.

Alica Ryba stellt sich in ihrem theoretischen Beitrag die Frage, welche Rolle unbewusste Prozesse im Coaching haben. Dazu bezieht sie sich zunächst auf eine neurowissenschaftlich gestützte Unterscheidung von Unbewusstem, Vorbewusstem und Bewusstem und auf ein Vier-Ebenen-Modell von Psyche und Persönlichkeit. In einer Übertragung auf Coaching zieht die Autorin, im Kontrast zum weit verbreiteten, kognitiv-reflexiven Verständnis von Coaching, zentrale Schlüsse zur Bedeutung von emotionalen Prozessen im Coaching. Weiter zeigt sie, warum vor allem bei tief greifenden Persönlichkeitsentwicklungen und zur Erhöhung der Wirksamkeit von Coaching vermehrt psychodynamische und hypnotherapeutische Methoden eingesetzt werden sollten. 
Bei der Begleitung von Veränderungsprozessen sind Coaches immer wieder mit den Beschränkungen eines rein sprachlich-kognitiven Austauschs konfrontiert. Unbewusste Anteile, die für die Veränderung von Klientinnen und Klienten von zentraler Bedeutung wären, werden oft gar nicht erschlossen. Inwiefern über den Einsatz von Bildmaterialien unbewusste Prozesse als Ressourcen zugänglich gemacht werden können, fragt sich in ihrem Beitrag Jasmin Messerschmidt. Auf der Grundlage einer Reihe von qualitativ ausgewerteten Interviews mit Coaches und Klientinnen oder Klienten zeigt sie, inwiefern Coaching-Prozesse mithilfe von Bildern wirksam unterstützt werden können.

Welche »Wirkfaktoren" einen empirisch nachweisbaren Einfluss auf den Erfolg von Einzel-Coachings haben, ist das Thema von Cornelia Tonhäuser. Sie hat dazu 24 empirische Studien ausgewertet und kommt zum Schluss, dass acht empirisch bestätigte Wirkfaktoren vorliegen, die sie drei Dimensionen zuordnet (Coach, Coaching-Prozess und Arbeitsbeziehung) und ausführlich vorstellt.

Julia Weber geht in ihrem Beitrag der Frage nach, wie Coaching-Ziele formuliert werden müssen, um besonders handlungswirksam zu sein. Unter Berücksichtigung der Unterscheidung von Verstand und Unbewusstem, verschiedenen Zieltypen und der Multiple-Code-Theorie von Bucci stellt Weber eine besondere Art von Zielen vor, die »Motto-Ziele«. Sie beschreibt die Wirkweise dieser bildhaft angereicherten Haltungsziele und zeigt, wie »Motto-Ziele« formuliert werden müssen und welche empirischen Belege - im Anschluss an eigene Untersuchungen - für die positive Wirkung dieses Zieltypus bereits vorliegen.

Sandra J. Schiemann, Christina Mühlberger, Maximilian D. Mühlberger, Isabell Braumandl und Eva Jonas prüfen in ihrem Beitrag, inwiefern in einem Coaching das von Deci und Ryan beschriebene Grundbedürfnis nach Autonomie und Selbstregulation - im Unterschied zu den Grundbedürfnissen nach Kompetenz und sozialer Eingebundenheit - in besonderer Weise erfüllt wird. Die Autorinnen und der Autor folgen dabei dem Verständnis von Coaching als Beratungsformat zur Erreichung selbstkongruenter Ziele. Sie stützen sich in ihrem Beitrag auf drei empirische Studien. Aufgrund ihrer Ergebnisse formulieren sie abschließend theoretische und praktische Implikationen.

Wieso schaffen es manche Menschen besser als andere, Lern- und Veränderungsimpulse aus Seminaren und Trainings umzusetzen? Ausgehend von dieser Frage, entwickelt Axel Koch faktorenanalytisch das "Transferstärke-Modell«. Es besteht aus vier psychologischen Faktoren, die - bei hohen Werten - im Anschluss an Seminare und Trainings zu einem hohen Lerntransfer führen. Der Beitrag stellt auch ein darauf aufbauendes Coaching-Programm vor. Dessen Idee besteht darin, je nach Faktoren-Ausprägung für die betroffenen Personen - insbesondere für diejenigen, die bei einzelnen Faktoren weniger hohe 
Werte ausweisen - maßgeschneiderte Maßnahmen abzuleiten, um zu generell höheren Lerntransferraten zu gelangen.

Laura Glauser stellt eine eigene ethnografische Studie vor, die nicht intendierte negative Wirkungen von Coaching untersucht. Eingebettet im vorherrschenden gesellschaftlichen Diskurs, den sie mit dem Begriff des neoliberalen »unternehmerischen Selbst" in Verbindung setzt, beschreibt sie am Beispiel des Career Coaching an Hochschulen, dass lösungsorientiertes Coaching nicht zwingend nur zu positiven Effekten bei den Coachees führt. Gerade wenn sich bei den Klienten und Klientinnen kein Erfolg einstellt, kann dies zu Selbstzweifeln führen. Als Ursache für solche Wirkungen sieht die Autorin vor allem die ungenügende Thematisierung struktureller und damit individuell kaum beeinflussbarer Faktoren im Coaching: dass es für junge Akademikerinnen und Akademiker zum Beispiel nicht so einfach ist, eine Stelle zu finden.

Eva Traut-Mattausch und Mirjam Zanchetta beschreiben in ihrem Beitrag, wie das »Impostor-Phänomen« mit Coaching gezielt bearbeitet werden kann. Mit diesem Begriff wird die Tatsache bezeichnet, dass jemand sich innerlich, obgleich objektive Beweise des Gegenteils vorliegen, als professionellen oder intellektuellen Hochstapler erlebt. Im Beitrag geben die beiden Autorinnen einen Überblick über die einschlägige Literatur zu diesem besonders bei promovierten Akademikerinnen verbreiteten Thema. Im zweiten Teil des Beitrags wird der konzeptionell-theoretische Rahmen vorgestellt, mit dem das Phänomen im Coaching bearbeitet werden kann.

Willem Jan Hofmans entwickelt ein Evaluationsmodell für FührungskräfteCoaching und überprüft es anhand eines quasiexperimentellen Designs mit dreißig Coachees. Er kommt zum Schluss, dass Coaching einen positiven Einfluss auf die Mindfulness der Coachees hat. Die Einschätzung der Leadership Effectiveness durch die Coachees selbst und ihre Vorgesetzten fiel nach dem Coaching ebenfalls signifikant höher aus. Dies ist als Beleg dafür zu werten, dass die Wirkungen eines Coachings nicht im eigentlichen "Empfänger « von Coaching eingekapselt bleiben, sondern auch im relevanten sozialen Umfeld wahrgenommen werden.

\section{Teil 2 - Forschungsrelevante Beiträge aus der Praxis}

Der Beitrag von Elke Berninger-Schäfer und Efriom Kineselassie greift die zunehmende Differenzierung von Themen, Settings und Zielgruppen im Coaching und die damit verbundene Segmentierung des Coaching-Markts auf. Die ständig wachsende Formatvielfalt wird anhand von zwei Beispielen, »Teamcoaching online « und »Walk and Talk« skizziert; im Anschluss werden relevante 
Format- und Setting-Faktoren präsentiert, auf deren Grundlage eine weiterführende Diskussion über »Formatkompetenz« geführt werden soll.

Wolfgang Eberling, Gery Bruederlin und Marion Alt geben in ihrem Beitrag Einblick in den "Laufbahnkoffer HR» (LKHR), ein Coaching-gestütztes Angebot für Weiterbildungsteilnehmende im Human-Resources-Bereich. Ziel des LKHR ist eine verbesserte Selbststeuerung von Teilnehmern und Teilnehmerinnen an Weiterbildungen und damit eine effektivere Nutzung der besuchten Angebote für die eigene berufliche Entwicklung. Neben inhaltlichen Komponenten des LKHR werden im Beitrag auch Bewertungen dieses Pilotprojekts durch Studierende diskutiert.

Niki Harramach und Nina Veličković berufen sich auf ihre langjährigen Erfahrungen in Evaluation seit den Coaching-Pionierzeiten in den 1980er Jahren. Sie kritisieren - aus Sicht der Praxis - die aktuellen wissenschaftlichen Evaluationsmodelle als zu komplex und plädieren dafür, auf Begriffe wie »Prozess- und Strukturqualität « zu verzichten. Stattdessen seien vermehrt die Ergebnisse von Coaching zu betrachten, aus der Sicht von Kundinnen und Kunden die einzige Qualität, die zähle. Auch aus juristischer Sicht sei es geboten, die Zielerreichung in den Mittelpunkt zu stellen.

Im Beitrag von Mathias Hofmann wird anhand eines Praxisfalls das aufschlussreiche Zusammenspiel von Coaching und Change-Management in einer größeren deutschen Stadtverwaltung dargestellt. Hauptziel des Projekts war die qualifizierende Entwicklung der Führungskräfte und die gleichzeitige Bewältigung strategischer Herausforderungen (Haushaltskonsolidierung, Umsetzung von großen Infrastrukturprojekten und Bildungsplanung). Im Sinne einer Fallstudie wird das Projekt im konkreten Ablauf, in den Zielen und Architekturen (Beratungsdesign) beschrieben, parallel werden die konkreten Beiträge der verschiedenen Akteure (Bürgermeister, Personalentwicklerin, externe Beratungspersonen und Führungspersonen) erläutert.

Vor dem Hintergrund der globalisierten und digitalisierten Arbeitswelt präsentieren Axel Klimek und Werner Stork aktuelle Herausforderungen, mit denen moderne Organisationen produktiv umgehen müssen. Konkret werden die "resiliente« und »agile« Organisation als Antwort auf diese Herausforderungen beschrieben. Dieses Organisationsmodell wurde von Wissenschaftlern der Hochschule Darmstadt zusammen mit Experten ausgewählter Unternehmen entwickelt. Ein besonderer Fokus liegt auf der Frage, welchen Beitrag eine Coaching-Kultur im Rahmen der beschriebenen Organisationsform leisten kann, worin diese besteht und wie sie im Kontext kultureller Veränderungsprozesse gefördert werden kann. 


\section{Literatur}

Birgmeier, B. (2006). Coaching und Soziale Arbeit: Grundlagen einer Theorie sozialpädagogischen Coachings. Weinheim: Juventa.

Böning, U., Fritschle, B. (2005). Coaching fürs Business. Was Coaches, Personaler und Manager über Coaching wissen müssen. Bonn: ManagerSeminare.

De Meuse, K. P., Dai, G., Lee, R. J. (2009). Evaluating the effectiveness of executive coaching: Beyond ROI? Coaching: An International Journal of Theory, Research and Practice, 2 (2), 117-134.

Deplazes, S. (2015). KaSyCo - Kategoriensystem zur Analyse von Coachingprozessen. Kassel: Kassel University Press.

Duncan, B. L., Miller, S. D., Wampold, B. E., Hubble, M. A. (Hrsg.) (2010). The Heart \& Soul of Change. Delivering What Works in Therapy ( $2^{\text {nd }}$ ed.). Washington D.C.: American Psychological Association.

Elliott, R. (2010). Psychotherapy change process research: Realizing the promise. Psychotherapy Research, 20 (2), 123-135.

Fietze, B. (2011). Chancen und Risiken der Coachingforschung - eine professionssoziologische Perspektive. In R. Wegener, A. Fritze, M. Loebbert (Hrsg.), Coaching entwickeln. Forschung und Praxis im Dialog (S. 24-33). Wiesbaden: VS Verlag für Sozialwissenschaften.

Fietze, B. (2015). Coaching auf dem Weg zur Profession? Eine professionssoziologische Einordnung. In A. Schreyögg, C. Schmidt-Lellek (Hrsg.), Die Professionalisierung von Coaching. Ein Lesebuch für den professionellen Coach (S. 3-21). Wiesbaden: Springer VS.

Fietze, B. (2016). Coaching in der reflexiven Moderne. Zwischen Komplexität und Handlungsfähigkeit. In R. Wegener, S. Deplazes, M. Hasenbein, H. Künzli, B. Uebelhart, A. Ryter (Hrsg.), Coaching als individuelle Antwort aufgesellschaftliche Entwicklungen. Kongressband (S. 36-44). Wiesbaden: VS Verlag für Sozialwissenschaften.

Fietze, B. (2017). Auf zwei Beinen läuft man besser. Professionelle Beratung braucht Forschung. News ÖVS, 1, o. S.

Geißler, H. (2016). Die Grammatik des Coachens - eine empirische Rekonstruktion. Wiesbaden: Springer.

Greif, S. (2014). Coaching und Wissenschaft - Geschichte einer schwierigen Beziehung. Organisationsberatung, Supervision, Coaching, 21 (3), 295-311.

Greif, S. (2016). Wie wirksam ist Coaching? Ein umfassendes Evaluationsmodell für Praxis und Forschung. In R. Wegener, M. Loebbert, A. Fritze (Hrsg.), Coaching-Praxisfelder. Forschung und Praxis im Dialog (2. Aufl., S. 161-182). Wiesbaden: Springer.

Greif, S. (2017). Researching Outcomes of Coaching. In T. Bachkirova, G. Spence, D. Drake (Eds.), The SAGE Handbook of Coaching (pp. 569-588). London: Sage.

Haan, E. de, Duckworth, A. (2012). Signalling a new trend in executive coaching outcome research. International Coaching Psychology Review, 8 (1), 6-19.

Jones, R. J., Woods, S. A., Guillaume, Y. R. F. (2015). The effectiveness of workplace coaching: A meta-analysis of learning and performance outcomes from coaching. Journal of Occupational and Organizational Psychology, 89 (2), 249-277.

Künzli, H. (2009). Wirksamkeitsforschung im Führungskräfte-Coaching. Organisationsberatung, Supervision, Coaching, 16 (1), 4-16.

Lippmann, E. (2007). Alles Coaching ... oder was? Forum Supervision, 29, 26-39.

Lippmann, E. (2015). Was macht einen Coach zum Coach? Weiterbildung und Qualifizierung. Coaching. Theorie \& Praxis, 1 (1), 51-60.

Loebbert, M. (2017). Coaching Theorie. Eine Einführung (2., aktual. Aufl.). Wiesbaden: Springer.

Möller, H., Kotte, S. (2011). Die Zukunft der Coachingforschung. Organisationsberatung, Supervision, Coaching, 18 (4), 445-456. 
Myers, A. C. (2014). A Multiple Perspective Analysis of a Coaching Session. PhD thesis, Oxford Brookes University, Oxford.

Schiersmann, C., Thiel, H.-U. (Hrsg.) (2012). Beratung als Förderung von Selbstorganisationsprozessen. Empirische Studien zur Beratung von Personen und Organisationen auf der Basis der Synergetik. Göttingen: Vandenhoeck \& Ruprecht.

Schreyögg, A. (2013). Die Zukunft des Coachings. In E. Lippmann (Hrsg.), Coaching. Angewandte Psychologie für die Beratungspraxis (3. Aufl., S. 406-422). Berlin: Springer.

Seel, H.-J. (2014). Beratung: Reflexivität als Profession. Göttingen: Vandenhoeck \& Ruprecht.

Sonesh, S. C., Coultas, C. W., Lacerenza, C. N., Marlow, S. L., Benishek, L. E., Sala, E. (2015). The power of coaching: a meta-analytic investigation. Coaching: An International Journal of Theory, Research and Practice, 8 (2), 73-95.

Stelter, R. (2014). A Guide to Third Generation Coaching. Narrative-Collaborative Theory and Practice. Dordrecht: Springer.

Theeboom, T., Beersmaa, B., Vianena, A. E. M. van (2013). Does coaching work? A meta-analysis on the effects of coaching on individual level outcomes in an organizational context. The Journal of Positive Psychology: Dedicated to furthering research and promoting good practice, 9 (1), 1-18.

Timulak, L. (2008). Research in Psychotherapy and Counselling. Los Angeles: Sage.

Wegener, R. (2017). Bedeutsame Momente im Coaching - Plädoyer für eine prozessorientierte Forschungslandkarte sowie Entwicklung und Erprobung eines aufgaben- und kommunikationstheoretischen Verfahrens zur Identifikation und Analyse »bedeutsamer Momente im Coaching«. Diss., Universität der Bundeswehr, Hamburg:

Wegener, R., Deplazes, S., Hasenbein, M., Künzli, H., Ryter, A., Uebelhardt, B. (Hrsg.) (2015). Coaching als individuelle Antwort auf gesellschaftliche Herausforderungen. Wiesbaden: Springer VS.

Wegener, R., Loebbert, M., Fritze, A. (Hrsg.) (2015). Zur Differenzierung von Handlungsfeldern im Coaching. Wiesbaden: Springer VS.

Wegener, R., Loebbert, M., Fritze, A. (Hrsg.) (2016a). Coaching-Praxisfelder. Forschung und Praxis im Dialog (2. Aufl.). Wiesbaden: Springer VS.

Wegener, R., Loebbert, M., Fritze, A. (Hrsg.) (2016b). Coaching und Gesellschaft. Forschung und Praxis im Dialog. Wiesbaden: Springer. 


\section{Teil 1}

Wissenschaftliche Fachbeiträge 
(c) 2018, Vandenhoeck \& Ruprecht $\mathrm{GmbH} \&$ Co. KG, Göttingen https://doi.org/10.13109/9783666402975 | CC BY-NC-ND 4.0 


\title{
Peter oder Petra?
}

\section{Die Rolle von Geschlecht und Führungsqualitäten in der Bewertung und Auswahl von Coaches}

\author{
Maximilian D. Mühlberger und Eva Traut-Mattausch
}

Viele sprechen von Coaching, als wäre immer klar, was damit genau gemeint ist. Dabei hat sich durch das große Interesse an Coaching, sowohl in der freien Wirtschaft als auch in der Forschung, eine Vielzahl an unterschiedlichen Coaching-Ansätzen und -Zielgruppen entwickelt. Nun stellt sich die Frage, ob sich Menschen von Coaches ein bestimmtes Bild machen und ob dieses die Bewertung und schließlich die Auswahl eines Coaches beeinflusst. Einen interessanten Erklärungsansatz bietet die Implizite Führungstheorie (IFT), die besagt, dass Personen einen bestimmten Prototyp einer Führungskraft im Kopf haben und dass dieser Prototyp wiederum die Bewertung und Zusammenarbeit mit der Führungskraft beeinflussen kann. In einer Studienreihe an der Universität Salzburg haben die Autorin und der Autor diese Theorie auf den Coaching-Kontext übertragen. Dabei konnten sie zeigen, dass Studienteilnehmerinnen und -teilnehmer erstens Coaches mit Eigenschaften, die eher Frauen zugeschrieben werden, assoziieren, zweitens transformationale und weibliche Coaches besser bewerten und eher engagieren würden und drittens diese Bevorzugung durch die Zuschreibung hoher Sensitivität, sowohl weiblicher als auch transformationaler Coaches, entsteht.

Die Nachfrage nach Coaching und das Interesse an Coaching-Forschung ist in den letzten Jahren kontinuierlich angestiegen; Coaching hat sich zum Milliardengeschäft entwickelt (ICF, 2012; Passmore \& Fillery-Travis, 2011; Stober \& Grant, 2006; Theeboom, Beersma \& van Vianen, 2014). Im Zuge dieses Booms hat sich eine Vielzahl an unterschiedlichen Ansätzen entwickelt, zum Beispiel psychodynamisches, lösungsorientiertes oder personenzentriertes Coaching; es sind Coachings für eine Vielzahl spezieller Anlässe und Zielgruppen entstanden, etwa Performance Coaching, Entwicklungs- oder Executive Coaching, genauso wie Lebens-, Gesundheits- oder Karriere-Coaching (Cox, Bachkirova \& Clutter- 
buck, 2014; Della Picca et al., 2013; Fischer et al., 2013). In der Folge haben sich mittlerweile viele Definitionen von Coaching entwickelt, die durchaus unterschiedliche Aspekte der Coaching-Tätigkeit hervorheben. Viele Definitionen beschreiben Coaching aber als lösungs- und ressourcenorientierten Prozess, der darauf abzielt, die Selbstreflexion anzuregen und die Erreichung von Zielen und persönliches Wachstum zu unterstützen (z. B. Grant \& Stober, 2006; Greif, 2008). Ergebnisse von de Haan, Culpin und Curd (2011) deuten darauf hin, dass die Nützlichkeit von Coaching nicht so sehr von einem bestimmten Ansatz oder spezifischen Techniken bestimmt wird, sondern eher durch allgemeinere Faktoren wie die Beziehung zwischen Coach und Coachee oder davon, wie gut Coaches sich in die Klientinnen und Klienten hineinversetzen können. Wenn es solche allgemeingültigen Faktoren für das Coaching gibt, gibt es dann auch so etwas wie einen Coach-Prototyp, der allgemein wichtige Eigenschaften von Coaches widerspiegelt?

\section{Implizite Führungstheorie}

Einen Ansatzpunkt, um dieser Frage nachzugehen, bietet die Implizite Führungstheorie (IFT), die zu erklären versucht, wie Mitarbeiter und Mitarbeiterinnen mit ihren Führungskräften interagieren (Epitropaki \& Martin, 2004; Wenqua, Chia \& Liluo, 2000). Die IFT besagt, dass Menschen eine bestimmte kognitive Kategorie oder einen Prototyp von »Führungskraft« besitzen, um andere als Führungskraft oder Nicht-Führungskraft zu identifizieren (Lord, de Vader \& Alliger, 1986). Dieser Prototyp besteht aus den impliziten Annahmen über Führungskräfte und deren allgemeine Eigenschaften (Phillips, 1984). Ausgehend von dieser impliziten Führungstheorie, die aus der Erfahrung mit Führungskräften entstanden ist, bilden sich Menschen ein Bild von ihrer Führungskraft (Epitropaki \& Martin, 2005). Dabei können die eigenen IFTs die Wahrnehmung von Führungskräften beeinflussen und zu selektiver Informationssuche führen (Kenney, Schwartz-Kenney \& Blascovich, 1996; Lord \& Maher, 1990). Diskrepanzen zwischen der eigenen IFT und einer aktuellen Führungskraft können zu negativen Konsequenzen, wie zum Beispiel schlechterem leader-member exchange führen (Epitropaki \& Martin, 2005). Zu den prototypischen Eigenschaften von Führungskräften zählen Sensitivität, Intelligenz, Einsatz und Dynamik. Zu den antiprototypischen Eigenschaften zählen Tyrannei und Maskulinität (Epitropaki \& Martin, 2004). Wir haben dieses Konzept auf das Coaching übertragen, da Coaches auch eine Art von Führungskräften sind - sie führen durch den Coaching-Prozess (Mühlberger \& Traut-Mattausch, 2015). Das bedeutet, 
dass Personen eine implizite Coaching-Theorie haben können, die dann wiederum die Bewertung und Auswahl von Coaches beeinflusst. Die Verbindung von Führungskräften und Coaches wird im nächsten Absatz deutlich, wenn wir die Eigenschaften betrachten, die für beide wichtig sind.

\section{Coach-Prototyp}

Einen ersten Ausgangspunkt, der ebenfalls aus der Führungsforschung kommt, liefern Mühlberger und Traut-Mattausch (2015), die den Coach als Führungskraft im Coaching-Prozess beschreiben und Überschneidungen zwischen Coaching und transformationaler Führung darlegen. Transformationale Führung durch den Coaching-Prozess kann dabei direkte positive Effekte sowohl auf die Coaching-Ergebnisse haben, wie zum Beispiel Selbstreflexion oder Ziel-Commitment (Mühlberger \& Traut-Mattausch, 2015), als auch auf wichtige vorausgehende Bedingungen, wie zum Beispiel Autonomieunterstützung oder Arbeitsbeziehung (Losch, Traut-Mattausch, Mühlberger \& Jonas, 2016). Auch ein Vergleich der Eigenschaften, die für Coaches und transformationale Führungskräfte wichtig sind, legt nahe, dass es Überschneidungen gibt. In welchen Eigenschaften sich Coaches und transformationale Führung überschneiden,

\begin{tabular}{|l|l|}
\hline Coaches & Transformationale Führungskräfte \\
\hline $\begin{array}{l}\text { haben ein hohes Einfühlungsvermögen } \\
\text { (Passmore, 2010; Steinke, 2015) }\end{array}$ & $\begin{array}{l}\text { sind einfühlsam } \\
\text { (Hetland \& Sandal, 2003) }\end{array}$ \\
\hline $\begin{array}{l}\text { Sind mitfühlend } \\
\text { (Kombarakaran, Yang, Baker \& Fernandes, } \\
\text { 2008) }\end{array}$ & $\begin{array}{l}\text { zeigen Mitgefühl } \\
\text { (Bass \& Steidlmeier, 1999) }\end{array}$ \\
\hline $\begin{array}{l}\text { können gut zuhören und eine vertrauens- } \\
\text { volle Beziehung herstellen } \\
\text { (Wasylyshyn, 2003) }\end{array}$ & $\begin{array}{l}\text { können akzeptierende und unter- } \\
\text { stützende Beziehungen herstellen } \\
\text { (van Rensburg \& Crous, 2000) }\end{array}$ \\
\hline $\begin{array}{l}\text { zeigen Empathie und Respekt } \\
\text { (Dagley, 2010; Steinke, 2015) }\end{array}$ & $\begin{array}{l}\text { haben eine warmherzige Persönlichkeit } \\
\text { (de Vries, 2008) }\end{array}$ \\
\hline \multicolumn{2}{|c|}{$\begin{array}{c}\text { Implicit-Leadership-Theory-Dimension } \\
\text { Sensitivität }\end{array}$} \\
(sympathisch, sensibel, einfühlsam, verstehend, \\
aufrichtig, herzlich, nachsichtig und hilfreich) \\
(Offermann, Kennedy \& Wirtz, 1994)
\end{tabular}

Abbildung 1: Überschneidung zwischen Coaches, transformationalen Führungskräften und der IFT Dimension Sensitivität 
wird in Abbildung 1 deutlich. Wie dabei zu erkennen ist, sind wichtige Eigenschaften für Coaches und transformationale Führung Sensibilität, Empathie und Verständnis. Diese Eigenschaften können in einer der Dimensionen der IFT, Sensitivität, zusammengefasst werden, die wiederum ein zentraler Baustein eines Coach-Prototyps sein könnte.

In der Führungsforschung konnte ein positiver Effekt von transformationaler Führung in unterschiedlichen Settings, Hierarchieebenen und unabhängig vom Geschlecht der Führungskraft gefunden werden (Judge \& Piccolo, 2004; Wang, Oh, Courtright \& Colbert, 2011).

Im Gegensatz dazu ist die Evaluation von männlichen und weiblichen Führungskräften von geschlechtsspezifischen Stereotypen abhängig (Eagly \& Karau, 2002). So konnte über viele Studien hinweg gezeigt werden, dass Führung eher mit männlichen Eigenschaften assoziiert wird und daher männliche Führungskräfte auch als effektiver wahrgenommen werden (Koenig, Eagly, Mitchell \& Ristikari, 2011). Ergebnisse aus qualitativen Studien von Gray und Goregaokar (2010) deuten allerdings darauf hin, dass es im Coaching eher andersherum ist, dass also weibliche Coaches eher bevorzugt werden. Klientinnen neigen dazu, weibliche Coaches auszuwählen, da diese als Rollenmodell für Erfolg angesehen werden. Auf der anderen Seite wählen Klienten eher weibliche Coaches, weil sie als geeigneter gesehen werden, um sensible und persönliche Themen zu besprechen. Auch ein Vergleich weiblicher Attribute wie sympathisch, verstehend oder herzlich (Bem, 1981; Holt \& Ellis, 1998) mit den Eigenschaften aus Abbildung 1 deutet darauf hin, dass Coaching eher mit weiblichen Eigenschaften zusammenhängt.

\section{Aktuelle Forschung}

In einer Forschungsreihe an der Universität Salzburg wurde untersucht, wie der Führungsstil von Coaches und ihr Geschlecht die Bewertung und Auswahl von Coaches beeinflusst (Mühlberger \& Traut-Mattausch, 2016). So wurden in unterschiedlichen Fallvignetten transformational, transaktional, nach dem Laissezfaire-Stil, demokratisch und autoritär führende Coaches und weibliche bzw. männliche Coaches beschrieben. Die Ergebnisse zeigen, dass Studienteilnehmer und -teilnehmerinnen transformational führende und weibliche Coaches als vertrauenswürdiger und effektiver bewerten. Zudem würden sie transformationale und weibliche Coaches eher engagieren und ihnen ein höheres Honorar zahlen. Diese positiven Effekte des Führungsstils und des Geschlechts von Coaches können dadurch erklärt werden, dass Personen den Coaches generell eher weibliche als männliche Attribute zuschreiben, wie zum Beispiel Feinfühlig- 
keit oder Empfindsamkeit. Diese können unter der IFT-Eigenschaft Sensitivität zusammengefasst werden. Sowohl weibliche als auch transformationale Coach-Profile wurden als sensitiver wahrgenommen, was dann wiederum zu einer besseren Bewertung führte. Die Ergebnisse deuten aber auch darauf hin, dass weibliche Coaches nicht immer besser bewertet werden. Es wurde kein Geschlechtereffekt entdeckt, wenn Klienten und Klientinnen an einem kurzen Video-Coaching teilnahmen. Hier zeigte sich nur der Effekt des transformationalen Führungsstils. Dies könnte daran liegen, dass Personen in relativ abstrakten Situationen - etwa bei einer Fallvignette - eher auf Stereotype/Prototype zurückgreifen und in relativ konkreten Situationen - in einem konkreten Coaching - eher auf Informationen aus der tatsächlich stattfindenden Interaktion achten (McCrea, Wieber \& Myers, 2012).

\section{Implikationen für die Praxis}

Die theoretischen Überlegungen und empirischen Ergebnisse können zu einem besseren Verständnis von Coaching und relevanten Eigenschaften und Verhaltensweisen für ein erfolgreiches Coaching beitragen. Für die Praxis lassen sich daraus vielfältige Schlussfolgerungen ziehen. Sie betreffen die Außendarstellung von Coaches, die Gestaltung von Auswahlprozessen und die persönliche Entwicklung von Coaches.

\section{Außendarstellung von Coaches}

Die Ergebnisse deuten darauf hin, dass Coaches transformationale Elemente nutzen sollten, um sich selbst und ihren Coaching-Prozess zu beschreiben. Dadurch kann eine Zuschreibung positiver Attribute erfolgen, die zu mehr Aufträgen und höherem Einkommen führen kann. Da aber viele Klienten oder Klientinnen und Firmen auf Weiterempfehlungen und einen guten Ruf vertrauen (Dagley, 2009; Wasylyshyn, 2003), ist es wichtig, auch von anderen als transformational wahrgenommen zu werden, sodass dies auch weitergetragen wird.

\section{Gestaltung von Auswahlprozessen}

Obwohl es Studien gib, die zeigen, dass eine Ähnlichkeit zwischen Coach und Coachee nicht zu besseren Coaching-Ergebnissen führt (Boyce, Jackson \& Neal, 2004; de Haan, Grant, Burger \& Eriksson, 2016), so könnte eine Übereinstimmung zwischen den Vorstellungen der Klientinnen und Klienten und dem 
Coaching-Ansatz des Coaches zu einer besseren Passung führen. Eine derartige Passung könnte den Auswahlprozess und das Zusammenführen von CoachKlient/-in-Beziehungen erleichtern und darüber hinaus vor allem zu Beginn eines Coaching-Prozesses bei der Klärung des Auftrags und Ablaufs helfen.

\section{Persönliche Entwicklung als Coach}

Daran anknüpfend, kann es auch für die persönliche und berufliche Entwicklung von Coaches wichtig sein, was sich Klienten und Klientinnen von ihnen erwarten. Wie sich in den Ergebnissen der Studienreihe widerspiegelt, scheint Sensitivität ein Faktor zu sein, der von potenziellen Klientinnen und Klienten geschätzt wird und der zu einer besseren Wahrnehmung und Bewertung von Coaches führt. Dem Bild oder Prototyp der Klienten oder Klientinnen zu entsprechen oder Diskrepanzen frühzeitig anzusprechen, könnte zu einer Klärung der Erwartungen beitragen und damit den Beziehungsaufbau und den Verlauf des Coachings positiv beeinflussen.

\section{Fazit}

Wie in einem unlängst erschienenen Beitrag (Traut-Mattausch, Mühlberger, Braumandl \& Jonas, 2016) wollen wir hier erneut darauf aufmerksam machen, dass die Führungsforschung interessante Ansatzpunkte für die Entwicklung und Weiterentwicklung von Coaching und Coaches liefern kann. Anhand der vorgestellten Ergebnisse ist zu erkennen, dass Personen einen transformationalen Coach besser bewerten und bevorzugen. Der gleiche Effekt zeigte sich aber nicht immer für weibliche Coaches. Es scheint also, als hätten Peter und Petra gute Chancen, solange sie transformational sind. In weiterer Forschung wäre es spannend zu untersuchen, wie eine implizite Coaching-Theorie aussehen könnte und welche Auswirkungen diese auf Coach, Klient/-in und den Coaching-Prozess hat.

\section{Literatur}

Bass, B. M., Steidlmeier, P. (1999). Ethics, character, and authentic transformational leadership behavior. The Leadership Quarterly, 10 (2), 181-217.

Bem, S. L. (1981). Gender schema theory: A cognitive account of sex typing. Psychological Review, 88, 354-364. 
Boyce, L. A., Jackson, R. J., Neal, L. J., (2004). Building successful leadership coaching relationships: Examining impact of matching criteria in a leadership coaching program. Unpublished manut script, U.S. Air Force Academy. www.dtic.mil/dtic/tr/fulltext/u2/a524818.pdf [10.12.2017].

Cox, E., Bachkirova, T., Clutterbuck, D. (2014). The complete handbook of coaching. London: Sage.

Dagley, G. R. (2010). Exceptional executive coaches: Practices and attributes. International Coaching Psychology Review, 5 (1), 63-80.

Della Picca, M., Böning, U., Baitsch, C., Lippmann, R., Werkmann-Karcher, B., Passavant, C. von, Guyer, J., Negri, C. (2013). Zielgruppen für Einzelcoaching. In E. Lippmann (Hrsg.), Coaching. Angewandte Psychologie für die Beratungspraxis (S. 107-220). Berlin: Springer.

Eagly, A. H., Karau, S. J. (2002).Role congruity theory of prejudice toward female leaders. Psychological Review, 109, 573-598.

Epitropaki, O., Martin, R. (2004). Implicit leadership theories in applied settings: Factor structure, generalizability and stability over time. Journal of Applied Psychology, 89 (2), 293-310.

Epitropaki, O., Martin, R. (2005). From ideal to real: A longitudinal study of the role of Implicit Leadership Theories on Leader-Member Exchanges and employee outcomes. Journal of Applied Psychology, 90 (4), 659-676.

Grant, A. M., Stober, D. (2006). Introduction. In D. Stober, A. M. Grant (Eds.), Evidence based coaching: Putting best practices to work for your clients (pp. 1-14). Hoboken, NJ: Wiley.

Greif, S. (2008). Coaching und ergebnisorientierte Selbstreflexion. Theorie, Forschung und Praxis des Einzel- und Gruppencoachings. Göttingen: Hogrefe.

Gray, D. E., Goregaokar, H. (2010). Choosing an executive coach: The influence of gender on the coach-coachee matching process. Management Learning, 41 (5), 525-544.

Haan, E. de, Culpin, V., Curd, J. (2011). Executive coaching in practice: What determines helpfulness for clients of coaching? Personnel Review, 40 (1), 24-44.

Haan, E. de, Grant, A. M., Burger, Y., Eriksson, P.-O. (2016). A large-scale study of executive and workplace coaching: The relative contributions of relationship, personality match, and selfefficacy. Consulting Psychology Journal: Practice and Research, 68 (3), 189-207.

Hausherr Fischer, A., Enderlit, L., Lippmann, E., Ullmann-Jungfer, G., Negri, C., Perrin, D., Dubois, M., Nussbaumer, D., Vogelauer, W., Kalt, M., Blume, L. Eck, C. D. (2013). Spezielle Anwendungsfelder und Fragestellungen. In E. Lippmann (Hrsg.), Coaching. Angewandte Psychologie für die Beratungspraxis (S. 221-368). Berlin: Springer.

Hetland, H., Sandal, G. M. (2003). Transformational leadership in Norway: Outcomes and personality correlates. European Journal of Work and Organizational Psychology, 12 (2), 147-170.

Holt, C. L., Ellis, J. B. (1998). Assessing the current validity of the Bem Sex-Role Inventory. Sex Roles, 39 (11), 929-941.

ICF (International Coach Federation) (2012). ICF Global Coaching Study. www.coachfederation. org/coachingstudy2012 [10.12.2017].

Judge, T. A., Piccolo, R. F. (2004). Transformational and transactional leadership: A meta-analytic test of their relative validity. Journal of Applied Psychology, 89 (5), 755-768.

Kenney, R. A., Schwartz-Kenney, B. M., Blascovich, J. (1996). Implicit leadership theories: Defining leaders described as worthy of influence. Personality and Social Psychology Bulletin, 22 (11), 1128-1143.

Koenig, A. M., Eagly, A. H., Mitchell, A. A., and Ristikari, T. (2011). Are leader stereotypes masculine? A meta-analysis of three research paradigms. Psychological Bulletin, 137 (4), 616-642.

Kombarakaran, F. A., Yang, J. A., Baker, M. N., Fernandes, P. B. (2008). Executive Coaching: It works! Consulting Psychology Journal: Practice and Research, 60 (1), 78-90.

Lord, R. G., Maher, K. J. (1990). Alternative information-processing models and their implications for theory, research, and practice. Academy of Management Review, 15 (1), 9-28. 
Lord, R. G., Vader, C. L. de, Alliger, G. M. (1986). A meta-analysis of the relation between personality traits and leadership perceptions: An application of validity generalization procedures. Journal of Applied Psychology, 71 (3), 402-410.

Losch, S., Traut-Mattausch, E., Mühlberger, M. D., Jonas, E. (2016). Comparing the effectiveness of individual coaching, self-coaching, and group training: How leadership makes the difference. Frontiers in Psychology, 7 (629).

McCrea, S. M., Wieber, F., Myers, A. L. (2012). Construal Level mindsets moderate self and social stereotyping. Journal of Personality and Social Psychology, 102 (1), 51-68.

Morgan, H., Harkins, P., Goldsmith, M. (2006). The Right Coach. In J. V. Gallos (Ed.), Organization development (pp. 433-444). San Francisco: Jossey-Bass.

Mühlberger, M. D., Traut-Mattausch, E. (2015). Leading to effectiveness: Comparing dyadic coaching and group coaching. The Journal of Applied Behavioral Science, 51 (2), 198-230.

Mühlberger, M. D., Traut-Mattausch, E. (2016). Evaluating coach profiles - The influence of transformational leadership, sensitivity, and gender. Unpublished manuscript, Department of Psychology, University of Salzburg.

Offermann, L., Kennedy, J. K., Wirtz, P. W. (1994). Implicit leadership theories: Content, structure, and generalizability. The Leadership Quarterly, 5 (1), 43-58.

Passmore, J. (2010). A grounded theory study of the coachee experience: The implications for training and practice in coaching psychology. International Coaching Psychology Review, 5 (1), 48-62.

Passmore, J., Fillery-Travis, A. (2011). A critical review of executive coaching research: A decade of progress and what's to come. Coaching: An International Journal of Theory, Practice and Research, 4 (2), 70-88.

Phillips, J. S. (1984). The accuracy of leadership ratings: A cognitive categorization perspective. Organizational Behavior and Human Performance, 33 (1), 125-138.

Rensburg, C. van, Crous, F. (2000). Die verband tussen sekere persoonlikheidseienskappe e transformasionele leierskap. Journal of Industrial Psychology, 26 (3), 39-46.

Steinke, I. (2015). Kompetenzanforderungen an Coaches. In A. Schreyögg, C. Schmidt-Lellek (Hrsg.), Die Professionalisierung von Coaching (S. 257-282). Wiesbaden: Springer.

Stober, D. R., Grant, A. M. (2006). Toward a contextual approach to coaching models. In D. R. Stober, A. M. Grant (Eds.), Evidence based coaching handbook: Putting best practices to work for your clients (pp. 355-366). New York, NY: Wiley.

Theeboom, T., Beersma, B., Vianen, A. v. (2014). Does coaching work? A meta-analysis on the effects of coaching on individual level outcomes in an organizational context. Journal of Positive Psychology, 9 (1), 1-18.

Traut-Mattausch, E., Mühlberger, M. D., Braumandl, I., Jonas, E. (2016). Wie führen Coaches? In R. Wegener, S. Deplazes, M. Hasenbein, H. Künkli, A. Ryter, B. Uebelhart (Hrsg.), Coaching als individuelle Antwort auf gesellschaftliche Entwicklungen (S. 447-454). Wiesbaden: Springer VS.

Vries, R. E. de (2008). What are we measuring? Convergence of leadership with interpersonal and non-interpersonal personality. Leadership, 4 (4), 403-417.

Wang, G., Oh, I. S., Courtright, S. H., Colbert, A. E. (2011). Transformational leadership and performance across criteria and levels: A meta-analytic review of 25 years of research. Group and Organization Management, 36 (2), 223-270.

Wasylyshyn, K. (2003). Executive coaching: An outcome study. Journal of Consulting Psychology, 55 (2), 94-106.

Wenquan, L., Chia, R. C., Liluo, F. (2000). Chinese implicit leadership theory. Journal of Social Psychology, 140 (6), 729-739. 


\title{
Wo führt das hin?
}

\section{Effekte des Coaching-Führungsstils auf das Erleben der Klientinnen und Klienten und den Coaching-Erfolg}

\author{
Sabine Losch und Eva Traut-Mattausch
}

\begin{abstract}
Aktuelle Forschungsbefunde zeigen auf, dass das transaktionale und transformale Führungsverhalten des Coaches zur Effektivität von Coaching beiträgt. In diesem Beitrag wird zunächst ein Überblick über das Führungsverhalten im Coaching-Prozess gegeben. Anschließend werden die Ergebnisse zweier Feldstudien dargestellt, in denen die Auswirkungen dieses Führungsstils auf die Klienten und Klientinnen und den Coaching-Erfolg untersucht wurden. Daraus werden Implikationen abgeleitet, wie durch den Führungsstil von Coaches der Coaching-Prozess und die Interaktion mit den Klientinnen und Klienten effektiv gestaltet werden kann.
\end{abstract}

Mittlerweile gibt es immer mehr Studien, die auf die Wirksamkeit von Coaching hindeuten. So konnte gezeigt werden, dass Coaching den Return on Investment erhöht (De Meuse, Dai \& Lee, 2009), die Leistung und Zielerreichung steigert, die Einstellung bezüglich Karriere und Arbeit positiv beeinflusst sowie Bewältigungsstrategien zum effektiven Umgang mit Stressoren fördert (Theeboom, Beersma \& van Vianen, 2014). Doch was ist in einem Coaching-Prozess passiert, durch den Klienten und Klientinnen gute Ergebnisse erzielt haben? Nachdem Befunde vermehrt darauf hindeuten, dass Coaching wirkt, liegt der Fokus der aktuellen Forschung nun auf den Faktoren, die Coaching erfolgreich machen (de Haan, Duckworth, Birch \& Jones, 2013). Bisher gibt es aber nur wenige Studien, die bei der Untersuchung von Wirkfaktoren theoriegeleitet vorgehen (z. B. de Haan et al., 2013; Gessnitzer \& Kauffeld, 2015; Ianiro, Schermuly \& Kauffeld, 2013; Losch, Traut-Mattausch, Mühlberger \& Jonas, 2016; Mühlberger \& Traut-Mattausch, 2015b; Sue-Chan, Wood \& Latham, 2012). Um unsere Frage zu beantworten, ist es nötig, einen Blick auf verwandte, bereits etablierte Forschungsdisziplinen zu werfen und deren Erkenntnisse auf den Bereich Coaching zu übertragen. Dies führt uns zu relevanten Theorien und Konstruk- 
ten und somit zu wissenschaftlich fundierten Erklärungen zur Wirkungsweise von Coaching (Cavanagh, Grant \& Kemp, 2005).

\section{Auf der Suche nach den Wirkfaktoren}

In der arbeits- und organisationspsychologischen Forschung wurde untersucht, welche Faktoren Leistung, Anstrengungsbereitschaft und Zufriedenheit von Menschen positiv beeinflussen. Dabei hat sich vor allem eine hohe Übereinstimmung zwischen den Merkmalen von Personen (z. B. psychologische Bedürfnisse, Ziele) und deren Umfeld (z. B. intrinsische Anreize einer Tätigkeit) als ausschlaggebend erwiesen (Edwards, 2008; Edwards, Caplan \& Harrison, 1998; Gagné \& Deci, 2005). Es ist lohnenswert, den Coaching-Prozess ebenfalls aus der Perspektive der Person-Umfeld-Passung zu betrachten, da sich dadurch zentrale Überlegungen eröffnen: Was brauchen Personen, die sich unmittelbar in einem Entwicklungs- und Veränderungsprozess befinden, um diesen Prozess erfolgreich zu bewältigen? Und wie können Coaches diesen Prozess konkret gestalten, damit Klientinnen und Klienten durch das Coaching positive Effekte erzielen?

\section{Transaktionales und transformationales Führungsverhalten des Coach}

Coaching ist ein systematischer Prozess, in dem Klientinnen und Klienten lernen, eigenverantwortlich über sich selbst zu reflektieren, ihre Ziele zu verwirklichen und ihre Leistung zu verbessern (Grant \& Stober, 2006). Die Rolle des Coaches als Prozess- und Methodenexperte (Schein, 2003) ist es dabei, die Klienten und Klientinnen in diesem Prozess zu unterstützen, ohne Lösungen vorzugeben (Mühlberger \& Traut-Mattausch, 2015b). Theoretische Überlegungen weisen darauf hin, dass die Interaktion zwischen Coach und Klient/-in Parallelen dazu aufweist, wie Führungskräfte die Entwicklung und Leistung ihrer Mitarbeitenden fördern (Kemp, 2009). Als Führung wird grundsätzlich ein zielgerichteter Interaktionsprozess verstanden, in dem ein gegenwärtiger Zustand strukturiert oder restrukturiert wird und die Motivation und Kompetenzen der Geführten verändert werden (Bass \& Bass, 2008). Obwohl sich Coaching und Führung in einigen Aspekten unterscheiden (Coaching: symmetrische Beziehung, Erreichen persönlich relevanter Ziele vs. Führungskontext: asymmetrische Beziehung, Erreichen von Organisationszielen), weisen die Definitionen auf bedeutsame Gemeinsamkeiten hin: Ziel ist die Veränderung eines gegenwärtigen Zustands hin zu einem gewünschten Zielzustand. Indem Coaches bzw. Führungskräfte die Interaktion und den Prozess systematisch gestalten, 
befähigen sie ihre Klientinnen und Klienten bzw. ihre Mitarbeitenden, die Veränderungsziele zu erreichen (Mühlberger \& Traut-Mattausch, 2015b).

In einer Studienreihe der Universität Salzburg konnte gezeigt werden, dass sich wesentliche Merkmale des transaktionalen und transformationalen Führungsstils auf die Verhaltensweisen adaptieren lassen, mit denen Coaches den Prozess der Zielerreichung strukturieren (Mühlberger, Losch, Traut-Mattausch \& Jonas, 2015; Mühlberger \& Traut-Mattausch, 2015a; Traut-Mattausch, Mühlberger \& Jonas, 2015). Dies beinhaltet zum einen, vor dem Coaching gegenseitige Erwartungen zu klären und Aufgaben klar zu verteilen (transaktionale Führung), und zum anderen, die Entwicklung und Leistung der Klienten und Klientinnen durch das Coaching zu fördern und sie zu motivieren, über sich hinauszuwachsen (transformationale Führung) (für eine ausführliche Diskussion zur Anwendung von Führungstheorien im Coaching vgl. Mühlberger \& Traut-Mattausch, 2015b; Traut-Mattausch, Mühlberger, Braumandl \& Jonas, 2016).

Eine für Coaching relevante Facette der transaktionalen Führung ist die leistungsorientierte Belohnung. Sie ist dadurch charakterisiert, dass gegenseitige Erwartungen, Rahmenbedingungen und Rollen geklärt werden. Beispielsweise macht ein Coach deutlich, dass es in der Verantwortung der Klientinnen und Klienten selbst liegt, Lösungen zu erarbeiten und ihre Ziele zu erreichen, dass sie dabei aber Unterstützung durch den Coach erfahren. ${ }^{1}$ Darüber hinaus weisen folgende Komponenten des transformationalen Führungsstils - individuelle Wertschätzung, intellektuelle Stimulierung und inspirierende Motivierung Gemeinsamkeiten mit den Techniken auf, die Coaches verwenden, um die persönliche Entwicklung der Klienten und Klientinnen zu fördern und diese zu unterstützen: Der Coach geht auf die individuellen Ziele, Bedürfnisse und Stärken der Klientinnen und Klienten ein und unterstützt sie dabei, ihre individuellen Potenziale und Entwicklungsfelder zu erkennen (individuelle Wertschätzung). Er regt Klienten und Klientinnen an, bisherige Prozesse aus einer neuen Perspektive zu beleuchten, eingefahrene Denkmuster zu hinterfragen und für Probleme innovative Lösungsansätze zu finden (intellektuelle Stimulierung). Zudem signalisiert der Coach Optimismus hinsichtlich der Zielerreichung und hilft den Klientinnen und Klienten dabei, eine optimistische Vision ihrer Zukunft zu formulieren (inspirierende Motivierung) (Losch et al., 2016; Mühlberger \& Traut-Mattausch, 2015b). Die empirischen Befunde der Studien zei-

1 Ein weiterer Aspekt dieser Facette - Belohnungen einsetzen, um das Einhalten von Verpflichtungen und Erwartungen zu fördern - wird im Führungskontext angewendet, ist im Coaching aber nicht gegeben. 
gen, dass das transaktionale und transformationale Führungsverhalten des Coaches die Wirksamkeit des Coachings positiv beeinflusst. Bisher ist aber noch unbekannt, warum das Führungsverhalten des Coaches für einen erfolgreichen Entwicklungs- und Veränderungsprozess der Klienten und Klientinnen förderlich ist.

\section{Effekte der transaktionalen und transformationalen Führung auf die Klientinnen und Klienten und das Coaching-Ergebnis}

In der Führungsliteratur gilt der positive Zusammenhang zwischen transaktionaler Führung und dem organisationalen Commitment, der Zufriedenheit und Leistung der Mitarbeitenden als gut belegt. Ebenso konnten positive Effekte der transformationalen Führung auf subjektive (z.B. Zufriedenheit, Arbeitsengagement) wie auch objektive (z. B. Leistung der Mitarbeitenden, Führungsoder Unternehmenserfolg) Erfolgskriterien gezeigt werden (DeGroot, Kiker \& Cross, 2000; Felfe, 2006; Judge \& Piccolo, 2004; Lowe, Kroeck \& Sivasubramaniam, 1996; Wang, Oh, Courtright \& Colbert, 2011). Darüber hinaus wurden die Prozesse untersucht, durch die effektive Führungskräfte Einstellung, Verhalten und Leistung ihrer Mitarbeitenden beeinflussen. Befunde weisen darauf hin, dass transformationales Führungsverhalten die Art und Weise, wie Mitarbeitende ihre Führungskraft, das Arbeitsumfeld und ihre Tätigkeit erleben, verändert (Shamir, House \& Arthur, 1993). Beispielsweise schaffen transformationale Führungskräfte ein Arbeitsumfeld, das die Autonomie der Mitarbeitenden fördert und ihre intrinsische Motivation steigert (Baard, Deci \& Ryan, 2004; Charbonneau, Barling \& Kelloway, 2001; Conchie, 2013; Gagné \& Deci, 2005; Hetland, Skogstad, Hetland \& Mikkelsen, 2011). Zudem bewirkt dieses Führungsverhalten, dass Mitarbeitende sich gerecht behandelt fühlen (Bacha \& Walker, 2013; Krafft, Engelbrecht \& Theron, 2004; Pillai, Schriesheim \& Williams, 1999). Der Zusammenhang zwischen den Führungsverhaltensweisen und den Erfolgskriterien konnte dabei durch die bewirkten Veränderungen im Erleben der Mitarbeitenden erklärt werden. Es darf angenommen werden, dass die dargelegten Prozesse im Coaching ebenfalls wirksam werden, wenn Coaches transaktionales und transformationales Führungsverhalten zeigen. Ausgehend von diesen Befunden, wurde daher empirisch untersucht, inwiefern das Führungsverhalten des Coaches das Erleben und Verhalten der Klienten oder Klientinnen im Coaching-Prozess beeinflusst. 


\section{Forschungsdesign und -ergebnisse}

Zwei Feldexperimente wurden durchgeführt, um die Effekte des Coach-Verhaltens auf das Erleben der Klienten und Klientinnen und den Coaching-Erfolg zu überprüfen. Zu diesem Zweck wurden jeweils verschiedene Interventionen konzipiert und in ihrer Wirksamkeit mit einer Kontrollgruppe verglichen. Die Interventionen wurden in Inhalt und Ablauf parallel gehalten, unterschieden sich jedoch in ihrem spezifischen Setting voneinander. Die Kontrollgruppe erhielt keine Intervention, sondern setzte sich nur Ziele und beantwortete Fragebögen.

In einer ersten Studie (Losch et al., 2016) wurden 84 Studierende randomisiert einem Einzelcoaching, Selbstcoaching, Gruppentraining oder der Kontrollgruppe zugeteilt. Inhalt der Interventionen war, das Aufschiebeverhalten von Studierenden zu reduzieren. Coaching hatte positive Effekte auf die Zufriedenheit, die Zielerreichung und das Aufschiebeverhalten. Das transaktionale und transformationale Führungsverhalten führte dazu, dass sich Klientinnen und Klienten durch den Coach in ihrer Autonomie, also in ihren eigenen Handlungen und Wahlmöglichkeiten, mehr unterstützt fühlten und dadurch im Zielerreichungsprozess intrinsisch motivierter waren. Die Wirkfaktoren waren dabei bedeutsam für den Coaching-Erfolg (vgl. Abbildung 1).

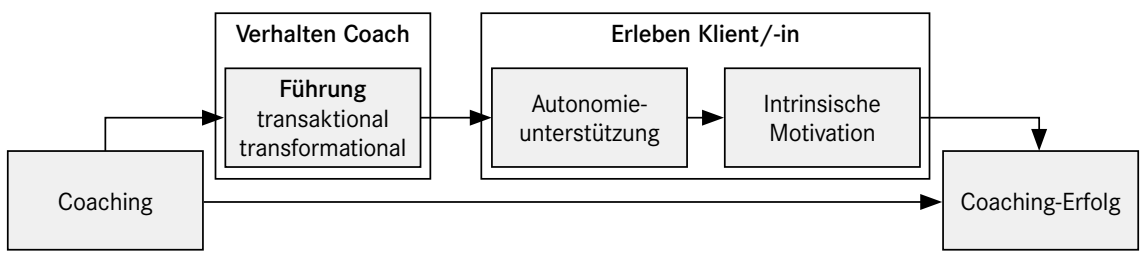

Abbildung 1: Der Zusammenhang zwischen Coaching und dem Coaching-Erfolg wird über das Coach-Verhalten (Führungsstil) und das Erleben der Klienten und Klientinnen (wahrgenommene Autonomieunterstützung und intrinsische Motivation) erklärt.

In einer weiteren Studie (Losch \& Traut-Mattausch, in prep.) wurden 63 Studierende randomisiert einem Einzelcoaching, einem Gruppentraining oder der Kontrollgruppe zugeteilt. Inhalt der Interventionen war, Studierende bei ihrer Karriereplanung zu unterstützen. Dabei konnte gezeigt werden, dass durch das Coaching und Training im Vergleich zur Kontrollgruppe die Vorstellungen und Ziele in Bezug auf die Karriere konkreter waren und die Zielerreichung höher war. Zudem erhöhte das transformationale Führungsverhalten des Coaches die wahrgenommene Gerechtigkeit, das heißt, die Klientinnen und Klienten erlebten in hohem Maße Wertschätzung und die Möglichkeit, Einfluss auf den Coa- 
ching-Prozess und dessen Ergebnis zu nehmen. Dies wirkte sich in der Folge darauf aus, wie Klienten und Klientinnen die Interaktion mit dem Coach erlebten: War die durch die Klientinnen und Klienten wahrgenommene Gerechtigkeit im Coaching-Prozess hoch, bewerteten sie die Qualität der gemeinsamen Arbeitsbeziehung positiver. Der positive Effekt von Coaching auf die Zielerreichung wurde durch das transformationale Führungsverhalten, die wahrgenommene Gerechtigkeit und Arbeitsbeziehung vermittelt (vgl. Abbildung 2).

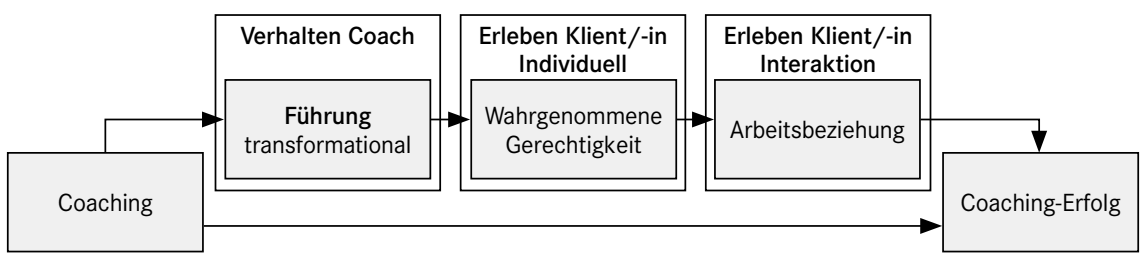

Abbildung 2: Der Zusammenhang zwischen Coaching und Coaching-Erfolg wird über das Coach-Verhalten (Führungsstil) und das Erleben der Klienten und Klientinnen (individuell: wahrgenommene Gerechtigkeit, Interaktion: Arbeitsbeziehung) erklärt.

\section{Implikationen}

Wie können Coaches den Coaching-Prozess gestalten, um Klienten und Klientinnen in ihrem Entwicklungs- und Veränderungsprozess effektiv und effizient zu unterstützen? Ausgehend von dieser Frage, wurde der Fokus in den vorliegenden Studien zunächst auf die Führungsrolle des Coaches gerichtet. Die Ergebnisse unserer beiden Feldexperimente bestätigen Forschungsbefunde, die zeigen, dass der transaktionale und transformationale Führungsstil einen wesentlichen Erfolgsfaktor für das Coaching darstellt (Mühlberger \& Traut-Mattausch, 2015b). Haltung und Vorgehensweise von Coaches - beispielsweise das Anerkennen von und Eingehen auf die Perspektiven, Bedürfnisse und Empfindungen (transformational) sowie das Äußern klarer Erwartungen (transaktional) - scheinen daher die Wirksamkeit des Coachings positiv zu beeinflussen. Zudem liefern die Ergebnisse ein tieferes Verständnis darüber, warum die Art und Weise, in der Coaches den Prozess der Zielerreichung strukturieren, das Coaching erfolgreich macht. Dabei hat sich unsere Annahme bestätigt, dass Führungsverhalten des Coaches und Coaching-Erfolg damit zusammenhängen, wie Klienten und Klientinnen den Coaching-Prozess und die Interaktion mit ihrem Coach erleben. 


\section{Effektive Gestaltung eines entwicklungsfördernden Settings im Coaching}

Wie die Ergebnisse der ersten Studie zeigen, scheinen für einen erfolgreichen Coaching-Prozess das Erleben von Autonomie und intrinsische Motivation wichtige Wirkfaktoren darzustellen. Die psychologische Forschung liefert empirisch gestützte Prinzipien zur Gestaltung des Umfelds, in dem Lern- und Veränderungsprozesse stattfinden. Zahlreiche Befunde aus den Bereichen Erziehung, Bildung, Beruf, Gesundheit und Sport belegen, dass Schüler und Schülerinnen, Mitarbeitende, Patienten und Patientinnen, Sportlerinnen und Sportler nachhaltig zur Verhaltensänderung motiviert werden, sofern ein hohes Maß an Autonomie gewährt wird (Gagné \& Deci, 2005; Ryan \& Deci, 2000, 2008; Ryan, Lynch, Vansteenkiste \& Deci, 2011; Vansteenkiste, Simons, Lens, Sheldon \& Deci, 2004). Daraus schließen wir, dass die Prinzipien effektiver Lernumgebungen auch auf den Coaching-Prozess übertragbar sind und daher in diesem Kontext weiter beforscht werden sollten. Eine weitere Bedingung, damit Menschen Veränderungen aktiv initiieren und umsetzen, ist, dass der Veränderungsprozess strukturiert ist. Struktur bezieht sich dabei auf eine klare, verständliche und konstruktive Kommunikation von Erwartungen und Feedback (Jang, Reeve \& Deci, 2010; Vansteenkiste et al., 2012). Die Forschung geht davon aus, dass Menschen dabei durch unterschiedliche Prozesse motiviert werden. Während die Autonomieunterstützung die wahrgenommene Autonomie und das Gefühl von Selbstbestimmung fördert, bewirkt Struktur primär die Wahrnehmung von Kompetenz und das Gefühl von Kontrolle über die eigenen Erfolge (Jang et al., 2010). Inwiefern es für einen wirksamen Coaching-Prozess wesentlich ist, das Bedürfnis der Klientinnen und Klienten nach Autonomie und Kompetenz zu erfüllen, und wie diese Faktoren mit den Führungsverhaltensweisen zusammenhängen, muss in zukünftigen Studien differenzierter untersucht werden.

\section{Effektive Gestaltung der Arbeitsbeziehung im Coaching}

Die Ergebnisse der zweiten Studie stützen neuere Forschungsbefunde, die besagen, dass eine tragfähige Arbeitsbeziehung auch für die Wirksamkeit von Coaching bedeutsam ist (Boyce, Jackson \& Neal, 2010; Dingman, 2004; Gessnitzer \& Kauffeld, 2015; McGovern et al., 2001). Zudem liefern unsere Befunde erstmals konkrete Hinweise darauf, wie Coaches die Entwicklung einer effektiven Arbeitsbeziehung beeinflussen. Führen Coaches transformational durch den Prozess, wird dieser von den Klientinnen oder Klienten als gerecht erlebt. Dies war maßgeblich dafür verantwortlich, dass Klienten und Klientinnen die 
Interaktion mit ihrem Coach als hilfreich und positiv erlebten. In der Psychotherapieforschung gilt die Qualität der Arbeitsbeziehung, wie sie letztlich von den Patientinnen und Patienten bewertet wird, als starker Prädiktor für den Therapieerfolg (Horvath \& Symonds, 1991). Um das Zusammenspiel zwischen dem Führungsverhalten des Coaches und dem Erleben der Klienten und Klientinnen für die Entstehung einer positiven Arbeitsbeziehung zu verstehen, ist weiterhin theoriegeleitete Forschung nötig. Beispielsweise zeigen Befunde, dass die Entwicklung einer sicheren Bindung zwischen Patient/-in und Therapeut/-in für den Therapieerfolg von großer Bedeutung ist (de Haan, 2012; Wiseman \& Tishby, 2014). Gleichermaßen wird in der Führungsliteratur die Rolle der transformationalen Führungskraft als »Bindungsperson« diskutiert (Davidovitz, Mikulincer, Shaver, Izsak \& Popper, 2007). Möglicherweise aktivieren transformationale Führungsverhaltensweisen des Coaches positive Bindungsschemata der Klienten und Klientinnen - also einen erhöhten Selbstwert und die Wahrnehmung, dass der Coach verfügbar ist und Unterstützung anbietet (Ainsworth, Blehar, Waters \& Wall, 1978; Hazan \& Shaver, 1987).

Zusammenfassend lassen sich aus den Befunden beider Studien die Führungsverhaltensweisen als theoretisch begründete Schlüsselkompetenzen von Coaches ableiten, durch die der Coaching-Prozess effektiv gestaltet werden kann. In diesem Zusammenhang sollten Führungskompetenzen in die Ausund Weiterbildung von Coaches integriert werden, um so Qualitätsstandards für die weitere Professionalisierung von Coaching zu setzen (Traut-Mattausch et al. 2016). Um ein umfassendes Verständnis der Wirkungsweise von Coaching zu erlangen, gilt es, in zukünftigen Studien weitere Effekte des transaktionalen und transformationalen Führungsverhaltens zu untersuchen.

\section{Fazit}

Unsere Forschungsergebnisse liefern ein tieferes Verständnis dafür, was in einem erfolgreichen Coaching-Prozess passiert. Wahrgenommene Autonomie, intrinsische Motivation, Gerechtigkeit und eine tragfähige Arbeitsbeziehung sind nicht nur als weitere isolierte Wirkfaktoren zu betrachten. Wir konnten aufzeigen, dass der Erfolg des Coachings vielmehr auf einem komplexen Wirkprozess beruht: Zum einen haben die von Coaches eingesetzten Methoden einen Einfluss darauf, wie Klienten oder Klientinnen das Coaching und die Interaktion mit ihrem Coach erleben, und zum Zweiten ist von diesem Erleben abhängig, ob das Coaching erfolgreich verläuft. Diese Befunde geben Coaches Wissen an die Hand, wie ein motivierendes Setting und eine tragfähige Arbeitsbeziehung 
mit ihren Klienten und Klientinnen effektiv gestaltet werden kann: indem sie transaktional und transformational den Prozess der Zielerreichung strukturieren.

\section{Literatur}

Ainsworth, M. S., Blehar, M. C., Waters, E., Wall, S. (1978). Patterns of attachment: A psychological study of the Strange Situation. Hillsdale, NJ: Erlbaum.

Baard, P. P., Deci, E. L., Ryan, R. M. (2004). Intrinsic need satisfaction: A motivational basis of performance and wellbeing in two work settings. Journal of Applied Social Psychology, 34 (10), 2045-2068.

Bacha, E., Walker, S. (2013). The relationship between transformational leadership and followers' perceptions of fairness. Journal of Business Ethics, 116 (3), 667-680. doi:10.1007/s10551-0121507-z.

Bass, B. M., Bass, R. (2008). The Bass handbook ofleadership: Theory, research, \& managerial applications ( $4^{\text {th }}$ ed.). New York, NY: Free Press.

Boyce, L. A., Jackson, R. J., Neal, L. J. (2010). Building successful leadership coaching relationships: Examining impact of matching criteria in a leadership coaching program. Journal of Management Development, 29 (10), 914-931. doi:10.1108/02621711011084231.

Cavanagh, M., Grant, A. M., Kemp, T. (2005). Evidence-based coaching: Theory, research and practice from the behavioural science. Vol. 1. Bowen Hills, QLD: Australian Academic Press.

Charbonneau, D., Barling, J., Kelloway, K. E. (2001). Transformational leadership and sports performance: The mediating role of intrinsic motivation. Journal of Applied Social Psychology, 31 (7), 1521-1534. doi:10.1111/j.1559-1816.2001.tb02686.x.

Conchie, S. M. (2013). Transformational leadership, intrinsic motivation, and trust: a moderated-mediated model of workplace safety. Journal of Occupational Health Psychology, 18 (2), 198-210. doi:10.1037/a0031805

Davidovitz, R., Mikulincer, M., Shaver, P. R., Izsak, R., Popper, M. (2007). Leaders as attachment figures: leaders' attachment orientations predict leadership-related mental representations and followers' performance and mental health. Journal of Personality and Social Psychology, 93 (4), 632-650. doi:10.1037/0022-3514.93.4.632.

De Meuse, K. P., Dai, G., Lee, R. J. (2009). Evaluating the effectiveness of executive coaching: Beyond ROI? Coaching: An International Journal of Theory, Research and Practice, 2 (2), 117 134. doi:10.1080/17521880902882413.

DeGroot, T., Kiker, D. S., Cross, T. C. (2000). A meta-analysis to review organizational outcomes related to charismatic leadership. Canadian Journal of Administrative Sciences, 17 (4), 356-371. doi:10.1111/j.1936-4490.2000.tb00234.x.

Dingman, M. E. (2004). The effects of executive coaching on job-related attitudes. Unpublizierte Dissertation. University, Virginia Beach, VA.

Edwards, J. R. (2008). Person-Environment Fit in Organizations: An Assessment of Theoretical Progress. The Academy of Management Annals, 2 (1), 167-230. doi:10.1080/19416520802211503.

Edwards, J. R., Caplan, R. D., Harrison, R. V. (1998). Person-environment fit theory: Conceptual foundations, empirical evidence, and directions for future research. In C. L. Cooper (Eds.), Theories of organizational stress (pp. 28-67). Oxford: Oxford University Press.

Felfe, J. (2006). Transformationale und charismatische Führung - Stand der Forschung und aktuelle Entwicklungen. Zeitschrift für Personalpsychologie, 5 (4), 163-176. doi:10.1026/16176391.5.4.163.

Gagné, M., Deci, E. L. (2005). Self-determination theory and work motivation. Journal of Organizational Behavior, 26 (4), 331-362. 
Gessnitzer, S., Kauffeld, S. (2015). The working alliance in coaching: why behavior is the key to success. The Journal of Applied Behavioral Science, 51 (2), 177-197. doi:10.1177/0021886315576407.

Grant, A. M., Stober, D. (2006). Introduction. In A. M. Grant, D. Stober (Eds.), Evidence based coaching: Putting best practices to work for your clients (pp. 1-14). Hoboken, NJ: Wiley.

Haan, E. de (2012). Back to basics II: How the research on attachment and reflective-self function is relevant for coaches and consultants today. International Coaching Psychology Review, 7 (2), 194-209.

Haan, E. de, Duckworth, A., Birch, D., Jones, C. (2013). Executive coaching outcome research: The contribution of common factors such as relationship, personality match, and self-efficacy. Consulting Psychology Journal: Practice and Research, 65 (1), 40-57.

Hazan, C., Shaver, P. R. (1987). Romantic love conceptualized as an attachment process. Journal of Personality and Social Psychology, 52 (3), 511-524.

Hetland, H., Skogstad, A., Hetland, J., Mikkelsen, A. (2011). Leadership and learning climate in a worksetting. European Psychologist, 16 (3), 163-173. doi:10.1027/1016-9040/a000037.

Horvath, A. O., Symonds, B. D. (1991). Relation between working alliance and outcome in psychotherapy: A meta-analysis. Journal of Counseling Psychology, 38 (2), 139-149. doi: org/10.1037/0022-0167.38.2.139.

Ianiro, P. M., Schermuly, C. C., Kauffeld, S. (2013). Why interpersonal dominance and affiliation matter: an interaction analysis of the coach-client relationship. Coaching: An International Journal of Theory, Research and Practice, 6 (1), 25-46. doi:10.1080/17521882.2012.740489.

Jang, H., Reeve, J., Deci, E. L. (2010). Engaging students in learning activities: It is not autonomy support or structure but autonomy support and structure. Journal of Educational Psychology, 102 (3), 588-600. doi:10.1037/a0019682.

Judge, T. A., Piccolo, R. F. (2004). Transformational and transactional leadership: a meta-analytic test of their relative validity. Journal of Applied Psychology, 89 (5), 755-768. doi:10.1037/00219010.89.5.755.

Kemp, T. J. (2009). Is coaching an evolved form of leadership? Building a transdisciplinary framework for exploring the coaching alliance. International Coaching Psychology Review, 4 (1), 105-110.

Krafft, P., Engelbrecht, A. S., Theron, C. C. (2004). The influence of transformational and transactional leadership on dyadic trust relationships through perceptions of fairnes. South African Journal of Industrial Psychology, 30 (1), 10-18. doi:10.4102/sajip.v30i1.142.

Losch, S., Traut-Mattausch, E. (in prep.). Establishing a powerful working alliance: Coach leadership behaviors as preconditions for effective coaching relationships.

Losch, S., Traut-Mattausch, E., Mühlberger, M. D., Jonas, E. (2016). Comparing the effectiveness of individual coaching, self-coaching, and group training: How leadership makes the difference. Frontiers in Psychology, 7, 629. doi:10.3389/fpsyg.2016.00629.

Lowe, K. B., Kroeck, K. G., Sivasubramaniam, N. (1996). Effectiveness correlates of transformational and transactional leadership: A meta-analytic review of the mlq literature. The Leadership Quarterly, 7 (3), 385-425. doi:10.1016/s1048-9843(96)90027-2.

McGovern, J., Lindemann, M., Vergara, M., Murphy, S., Barker, L., Warrenfeltz, R. (2001). Maximizing the impact of executive coaching: Behavioral change, organizational outcomes, and return on investment. Manchester Review, 6 (1), 1-9.

Mühlberger, M. D., Losch, S., Traut-Mattausch, E., Jonas, E. (2015). The role of leadership in different interventions against procrastination. Unpubliziertes Manuskript. Department of Psychology, University of Salzburg, Austria.

Mühlberger, M. D., Traut-Mattausch, E. (2015a). The evaluation of different leadership behavior of coaches. Department of Psychology, University of Salzburg, Austria.

Mühlberger, M. D., Traut-Mattausch, E. (2015b). Leading to effectiveness: Comparing dyadic coaching and group coaching. Journal of Applied Behavioral Science, 51 (2), 198-230. doi:10.1177/0021886315574331. 
Pillai, R., Schriesheim, C. A., Williams, E. A. (1999). Fairness perceptions and trust as mediators for transformational and transactional leadership: A two-sample study. Journal of Management, 25 (6), 897-933. doi:10.1177/014920639902500606.

Ryan, R. M., Deci, E. L. (2000). Self-determination theory and the facilitation of intrinsic motivation, social development, and well-being. American Psychologist, 55 (1), 68-78.

Ryan, R. M., Deci, E. L. (2008). A self-determination approach to psychotherapy: The motivational basis for effective change. Canadian Psychology, 49 (3), 186-193.

Ryan, R. M., Lynch, M., Vansteenkiste, M., Deci, E. L. (2011). Motivation and autonomy in counseling. psychotherapy, and behavior change: A look at theory and practice. The Counseling Psychologist, 39 (2), 193-260. doi:10.1177/0011000009359313.

Schein, E. H. (2003). Prozessberatung für die Organisation der Zukunft: Der Aufbau einer helfenden Beziehung (2. Aufl.). Bergisch-Gladbach: EHP.

Shamir, B., House, R. J., Arthur, M. B. (1993). The motivational effects of charismatic leadership: A self-concept based theory. Organization Science, 4 (4), 577-594. doi:10.1287/orsc.4.4.577.

Sue-Chan, C., Wood, R. E., Latham, G. P. (2012). Effect of a Coach's Regulatory Focus and an Individual's Implicit Person Theory on Individual Performance. Journal of Management, 38 (3), 809-835. doi:10.1177/0149206310375465.

Theeboom, T., Beersma, B., Vianen, A. van (2014). Does coaching work? A meta-analysis on the effects of coaching on individual level outcomes in an organizational context. The Journal of Positive Psychology, 9 (1), 1-18. doi:10.1080/17439760.2013.837499.

Traut-Mattausch, E., Mühlberger, M. D., Braumandl, I., Jonas, E. (2016). Wie führen Coaches? In R. Wegener, S. Deplazes, M. Hasenbein, H. Künzli, A. Ryter, B. Uebelhart (Hrsg.), Coaching als individuelle Antwort auf gesellschaftliche Entwicklungen (S. 447-454). Wiesbaden: Springer VS.

Traut-Mattausch, E., Mühlberger, M., Jonas, E. (2015). The difference between Coaching, Supervision, and Training. Unpubliziertes Manuskript. Department of Psychology, University of Salzburg, Austria.

Vansteenkiste, M., Sierens, E., Goossens, L., Soenens, B., Dochy, F., Mouratidis, A., Aelterman, N., Haerensa, L., Beyers, W. (2012). Identifying configurations of perceived teacher autonomy support and structure: Associations with self-regulated learning, motivation and problem behavior. Learning and Instruction, 22 (6), 431-439. doi:10.1016/j.learninstruc.2012.04.002.

Vansteenkiste, M., Simons, J., Lens, W., Sheldon, K. M., Deci, E. L. (2004). Motivating learning, performance, and persistence: the synergistic effects of intrinsic goal contents and autonomy-supportive contexts. Journal of Personality and Social Psychology, 87 (2), 246-260. doi:10.1037/0022-3514.87.2.246.

Wang, G., Oh, I. S., Courtright, S. H., Colbert, A. E. (2011). Transformational leadership and performance across criteria and levels: A meta-analytic review of 25 years of research. Group \& Organization Management, 36 (2), 223-270. doi:10.1177/1059601111401017.

Wiseman, H., Tishby, O. (2014). Client attachment, attachment to the therapist and client-therapist attachment match: How do they relate to change in psychodynamic psychotherapy? Psychotherapy Research, 24 (3), 392-406. doi:10.1080/10503307.2014.892646. 


\section{Die Bedeutung von Medien für die Qualität von Coaching}

Harald Geißler

Bereits ein flüchtiger Blick auf den Coaching-Diskurs zeigt, dass es bisher kaum Literatur zu Coaching-Medien gibt und dass völlig unklar ist, wie sie zu definieren sind. Um die Bedeutung von Medien für die Qualität von Coaching einzuschätzen, sind einige Vorarbeiten notwendig. Es wird hier deshalb zunächst versucht, Coaching-Medien konzeptionell zu umreißen und zu definieren, um auf dieser Grundlage zu klären, welche Erscheinungsformen es gibt und aus welchen Komponenten Coaching-Medien zusammengesetzt sind. So vorbereitet, wird dann am Beispiel eines ausgewählten Coaching-Mediums mit Bezug auf das Kriterium der Prozesswirksamkeit diskutiert, welche Bedeutung seine verschiedenen Komponenten für die Qualität von Coaching-Prozessen haben.

Unser Leben wird in den letzten Jahren zunehmend von den modernen Medien bestimmt. Es stellt sich deshalb die Frage, welche Bedeutung diese modernen und auch die traditionellen - Medien für Coaching und seine Qualität haben. Die Beantwortung der Frage setzt allerdings eine klare Vorstellung darüber voraus, was Coaching-Medien sind. Im Folgenden soll deshalb zunächst eine konzeptionell reflektierte Definition entwickelt und im zweiten Abschnitt ein Kategoriensystem für die Erfassung der verschiedenen Medien vorgestellt werden, die im Coaching genutzt werden können. Auf dieser Grundlage werden im dritten Abschnitt Plausibilitätseinschätzungen und Hypothesen zur Wirksamkeit der verschiedenen Medien diskutiert und am Beispiel des »Virtuellen Coachings (VC) illustriert.

\section{Was sind Coaching-Medien? - Definition und konzeptionelle Klärung}

Obwohl in den letzten zehn Jahren die Anzahl praxisnaher wie auch wissenschaftlicher Publikationen über Coaching gestiegen ist, sodass sich die Literatur heute kaum mehr überblicken lässt (Grant, 2006), gibt es nur sehr wenig 
Literatur über Coaching-Medien. In Standardwerken wie in dem von Rauen (2005) herausgegebenen "Handbuch Coaching «, im von Cox, Bachkirova und Clutterbuck (2010) publizierten »Complete Handbook of Coaching « und in der von Passmore, Peterson und Freire (2013) veröffentlichten »Psychology of Coaching and Mentoring « findet sich kein einziger Beitrag über Coaching-Medien, und - was noch schwerer wiegt - auch in den Sachregistern dieser Werke sucht man vergeblich nach dem Stichwort »Medien«.

Um zu klären und zu definieren, was Coaching-Medien sind, erweist sich ein Klassiker der erziehungswissenschaftlichen Literatur als hilfreich, nämlich der Aufsatz: »Unterricht - Analyse und Planung« von Wolfgang Schulz (1965). Sein Grundgedanke ist, dass unterrichtliches Handeln durch Entscheidungen bestimmt wird, die sich auf die vorliegenden Bedingungen und dabei auf vier grundlegende Entscheidungsfelder beziehen müssen: auf Intentionen, Inhalte, Methoden und Medien. Diese Entscheidungen wirken ursächlich aufeinander ein und implizieren Vorentscheidungen, die die anschließende Entscheidungsfreiheit einschränken (Blankertz, 1969).

Wenn man davon ausgeht, dass Entscheidungen nicht nur darauf gerichtet sind, was man tut oder unterlässt, sondern auch darauf, wie man die vorliegende Wirklichkeit versteht, wird zweierlei erkennbar: erstens, dass die pädagogische Wirklichkeit vier grundlegende Dimensionen aufweist, nämlich eine inhaltlich-gegenstandsbezogene, eine prozessual-methodische, eine normativ-intentionale und eine mediale Dimension, und zweitens dass diese Dimensionen aufs Engste miteinander verbunden sind.

Daraus folgt, dass die pädagogischen Akteure bestimmte Realitätsphänomene einerseits als »Medien « kategorisieren, dass aber die Phänomene darüber hinaus auch eine inhaltlich-gegenstandsbezogene, eine prozessual-methodische und eine normativ-intentionale Dimension haben.

Diese Überlegungen lassen sich auf Coaching übertragen. Denn Inhalte im Coaching sind die vorliegende Klientenproblematik und ihre Lösungsmöglichkeiten, die jenseits der Sphäre des Coachings liegen und deshalb im Coaching mithilfe von Coaching-Medien repräsentiert werden müssen. Für diesen Zweck bieten sich verschiedene Basismedien an: die mündliche Kommunikation im Hier und Jetzt, synchrone Video-Kommunikation und synchroner Text-Chat oder aber die asynchrone Kommunikation mithilfe von Voice-Mails, Videonachrichten oder E-Mails. Die Wahl des jeweiligen Mediums impliziert prozessual-methodische und normativ-intentionale Vorentscheidungen. Die Absicht, den Klienten oder die Klientin spontan-spielerisch zu alternativen Denkmöglichkeiten anzuregen, lässt sich zum Beispiel mit Video-Coaching leichter erreichen als mit E-Mail-Coaching; und auch methodisch zeichnet 
sich E-Mail-Coaching durch ganz andere Chancen und Risiken aus als VideoCoaching.

Entsprechendes gilt auch für die Coaching-Problemlösungsmedien, mit denen die Basismedien angereichert werden können. Zum Beispiel bietet sich an, die vorliegende Coaching-Problematik und ihre Lösungsmöglichkeiten zusätzlich auf einem Flipchart, einem Whiteboard oder auf Karteikarten mithilfe von Stichworten, Skizzen oder Bildern oder von Materialien wie etwa Steinen, Holzfiguren, formbarem Knetgummi u. Ä. (Schreyögg, 2012) darzustellen.

\section{Welche Formen von Coaching-Medien gibt es?}

Wenn also Coaching-Medien sich auf die sinnlich wahrnehmbaren Phänomene beziehen, die als Träger nicht nur medialer, sondern auch inhaltsbezogener, methodischer und normativ-intentionaler Bedeutungen fungieren, stellt sich die Frage, um welche Phänomene es sich im Einzelnen handelt.

Mit Bezug auf die Coaching-Basismedien lassen sich diesbezüglich sechs Kommunikationskomponenten unterscheiden, nämlich

- linguistisches Verhalten (Wortwahl und Satzkomposition),

- paralinguistisches Verhalten (Sprechgeschwindigkeit, Lautstärke, Klangbildung, Artikulation, Betonungen, Sprechdynamik, Pausen und Verzögerungen, Laute wie zum Beispiel »AAhh" und »Oohh«, aber auch Lachen, Stöhnen u. Ä.),

- wechselseitiger Blickkontakt,

- nonverbale Körperexpressionen (Gesichtsausdruck, Körperkontakt, Körperhaltung, Körperbewegungen und die Positionierung des Körpers im Raum),

- die äußere Erscheinung der Interaktionspartner (Geschlecht, Alter, Gesichtszüge, ethnische Merkmale, Körpergröße und -umfang, Outfit wie z. B. Kleidung, Schmuck, Parfüm und Haarschnitt bzw. Frisur)

- und schließlich der Coaching-Kontext (also entweder die Räumlichkeiten des Klienten oder des Coaches, der Arbeitsplatz der Klientin, Outdoor Sites wie z. B. der Garten des Coaches oder ein Spaziergang in der Natur).

Für die Komposition der Coaching-Problemlösungsmedien bieten sich hingegen zwölf verschiedene Komponenten an, nämlich die Stimme, vorgegebene und gestaltbare Texte, Zeichen/Symbole und Problemlösungskontexte sowie vorgegebene, statisch oder dynamisch gestaltbare Menschen bzw. Tiere.

Die Medienkomponenten der Kommunikations- und Problemlösungsmedien sind auf unterschiedliche Weise durch die Sinnesaktivitäten des Sehens, 
Hörens, Tastens bzw. Fühlens und Riechens wahrnehmbar, wobei anzumerken ist, dass das Sehen sich zum einen auf 3-D-Visualität und zum anderen auf 2-D-Visualität bezieht. Die Sinnesdaten können auf natürliche Weise, das heißt nichtelektronisch (also z. B. mithilfe der menschlichen Stimme), oder elektronisch, also etwa als E-Mail-Text oder als Word-Dokument, erzeugt und vermittelt werden. In Tabelle 1 werden diese beiden Alternativen durch die Buchstabenkombinationen NEL (= nichtelektronisch) und EL (= elektronisch) abgekürzt.

Tabelle 1: Erscheinungsmerkmale der Coaching-Basismedien und der Coaching-Problemlösungsmedien. Die weißen Felder markieren bereits vorliegende Erscheinungsmerkmale, die dunkelgrauen Felder nicht denkmögliche Erscheinungsmerkmale, und die hellgrauen Felder markieren technisch noch nicht entwickelte Erscheinungsmerkmale. EL = elektronisch, NEL = nichtelektronisch, SYN = synchron, ASY = asynchron

\begin{tabular}{|c|c|c|c|c|c|c|c|c|c|c|c|c|c|c|c|c|c|c|c|c|c|}
\hline \multirow[b]{3}{*}{ 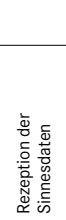 } & \multirow[b]{3}{*}{ 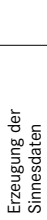 } & & & \multicolumn{6}{|c|}{ Basismedien } & \multicolumn{12}{|c|}{ Problemlösungsmedien } \\
\hline & & \multirow{2}{*}{\multicolumn{2}{|c|}{ 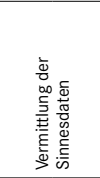 }} & \multirow[b]{2}{*}{ 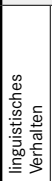 } & \multirow{2}{*}{ 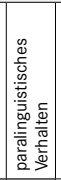 } & \multirow[b]{2}{*}{ 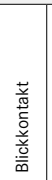 } & \multirow{2}{*}{ 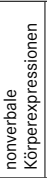 } & \multirow{2}{*}{ 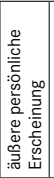 } & \multirow{2}{*}{ 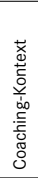 } & \multicolumn{3}{|c|}{ Texte } & \multicolumn{2}{|c|}{$\begin{array}{l}\text { Zeichen } \\
\text { Symbole }\end{array}$} & \multicolumn{2}{|c|}{$\begin{array}{l}\text { Gegen- } \\
\text { stände und } \\
\text { Pflanzen }\end{array}$} & \multicolumn{3}{|c|}{$\begin{array}{l}\text { Menschen und } \\
\text { Tiere }\end{array}$} & \multicolumn{2}{|c|}{$\begin{array}{c}\text { Problem- } \\
\text { lösungs- } \\
\text { kontext }\end{array}$} \\
\hline & & & & & & & & & & 密 & 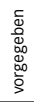 & 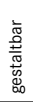 & 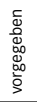 & 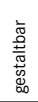 & 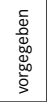 & 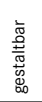 & 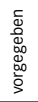 & 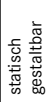 & 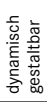 & 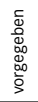 & 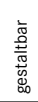 \\
\hline \multirow{6}{*}{ 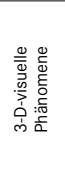 } & NEL & NEL & SYN & & & & & & & & & & & & & & & & & & \\
\hline & NEL & NEL & ASY & & & & & & & & & & & & & & & & & & \\
\hline & NEL & $\mathrm{EL}$ & SYN & & & & & & & & & & & & & & & & & & \\
\hline & NEL & EL & ASY & & & & & & & & & & & & & & & & & & \\
\hline & EL & EL & SYN & & & & & & & & & & & & & & & & & & \\
\hline & EL & EL & ASY & & & & & & & & & & & & & & & & & & \\
\hline \multirow{6}{*}{ 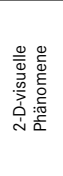 } & NEL & NEL & SYN & & & & & & & & & & & & & & & & & & \\
\hline & NEL & NEL & ASY & & & & & & & & & & & & & & & & & & \\
\hline & NEL & EL & SYN & & & & & & & & & & & & & & & & & & \\
\hline & NEL & EL & ASY & & & & & & & & & & & & & & & & & & \\
\hline & EL & EL & SYN & & & & & & & & & & & & & & & & & & \\
\hline & EL & EL & ASY & & & & & & & & & & & & & & & & & & \\
\hline \multirow{6}{*}{ 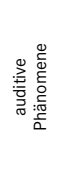 } & NEL & NEL & SYN & & & & & & & & & & & & & & & & & & \\
\hline & NEL & NEL & ASY & & & & & & & & & & & & & & & & & & \\
\hline & NEL & EL & SYN & & & & & & & & & & & & & & & & & & \\
\hline & NEL & EL & ASY & & & & & & & & & & & & & & & & & & \\
\hline & EL & EL & SYN & & & & & & & & & & & & & & & & & & \\
\hline & EL & EL & ASY & & & & & & & & & & & & & & & & & & \\
\hline \multirow{2}{*}{$\begin{array}{l}\text { taktile } \\
\text { Phän. }\end{array}$} & NEL & NEL & SYN & & & & & & & & & & & & & & & & & & \\
\hline & NEL & NEL & ASY & & & & & & & & & & & & & & & & & & \\
\hline \multirow{2}{*}{$\begin{array}{l}\text { olfaktor. } \\
\text { Phän. }\end{array}$} & NEL & NEL & SYN & & & & & & & & & & & & & & & & & & \\
\hline & NEL & NEL & ASY & & & & & & & & & & & & & & & & & & \\
\hline
\end{tabular}

$\mathrm{Zu}$ diesen beiden Merkmalen von Medienkomponenten kommt der Aspekt der Zeitlichkeit hinzu. Dieser gestaltet sich aber bei den Kommunikationsmedien anders als bei den Problemlösungsmedien. Bei den Coaching-Basismedien bedeutet Synchronizität (SYN), dass die Rezeption einer Botschaft durch den Empfänger zeitgleich mit der von einem Sender vorgenommenen Erstellung erfolgt, und Asynchronizität (ASY), dass diesbezüglich eine Zeitdifferenz vor- 
liegt. Bei den Coaching-Tools bedeutet Synchronizität (SYN) hingegen, dass die Auseinandersetzung der Klientin mit einem Coaching-Tool innerhalb des Dialogs erfolgt, den sie mit dem Coach - synchron oder asynchron - führt. Eine derartig dialogintegriert-synchrone Tool-Nutzung liegt nicht nur dann vor, wenn die Klientin während eines Face-to-Face- oder auch eines TelefonCoachings aus Knetgummi ihre Lebensvision formt, sondern auch dann, wenn sie diese Arbeit zu Hause durchführt, sich dabei filmt und das Video ihrem Coach zuschickt oder wenn sie während einer solchen »Hausaufgabe« die durch sie ausgelösten Erfahrungen und Erkenntnisse ihrem Coach per Voice-Mail oder E-Mail mitteilt.

Liegt hingegen eine deutliche Zeitdifferenz vor zwischen der - als SelbstCoaching vollzogenen - Auseinandersetzung des Klienten mit dem CoachingTool und der sich darauf beziehenden Kommunikation mit dem Coach, wird das Tool sequenziell-asynchron (ASY) genutzt.

\section{Prozesswirksamkeit von Coaching-Medien}

\subsection{Von der Ergebnis- zur Prozessforschung}

Um die Qualität von Coaching einzuschätzen, bemüht sich die neuere Coaching-Forschung um empirische Rekonstruktion sogenannter Wirkfaktoren bzw. Wirksamkeitsfaktoren (Greif, Schmidt \& Thamm, 2012; Greif, 2015; Wastian, 2015). Damit sind im Coaching-Prozess reliabel beobachtbare Phänomene vor allem des Coach-Verhaltens gemeint, deren Erscheinungshäufigkeit mit den Beurteilungen korreliert, welche die Klientinnen und Klienten nach Abschluss des Coachings bezüglich ihrer Zufriedenheit und/oder der Zielerreichung des Coachings formulieren. Ganz unabhängig davon, wie weitgehend man sich auf derartige subjektive Beurteilungen verlässt oder ob man zusätzlich auch die Beurteilungen von Personen aus dem Umfeld des Klienten oder der Klientin, also zum Beispiel ihrer Vorgesetzten, hinzuzieht: Das wesentliche Merkmal dieser Forschung ist, dass die Qualität von Coaching an Beurteilungen des Coaching-Outcomes gebunden wird, die nach Abschluss des Coachings erhoben werden.

Das ist nicht unproblematisch. Denn das Coaching ist ja nicht die einzige Ursache für den Coaching-Outcome. Es ist deshalb recht spekulativ, von Erfolgswirkfaktoren (Greif et al., 2012; Greif, 2015) zu sprechen. Etwas bescheidener sollte man sie vielleicht als Erfolgswirksamkeitsfaktoren oder als potenzielle Erfolgswirkfaktoren (Wastian, 2015, S. 301) bezeichnen. 
Auf der Grundlage dieses Forschungsdesigns kommt Siegfried Greif (Greif et al., 2012; Greif, 2015) zu dem Ergebnis, dass für einen positiven CoachingOutcome folgende - wie er sie nennt - Wirkfaktoren von zentraler Bedeutung sind: (1) Wertschätzung und emotionale Unterstützung, (2) Affektaktivierung und -kalibrierung, (3) ergebnisorientierte Problemreflexion, (4) ergebnisorientierte Selbstreflexion, (5) Zielklärung, (6) Ressourcenaktivierung und (7) Umsetzungsunterstützung. Bezogen auf die Frage nach der Bedeutung der Coaching-Medien für die Qualität von Coaching, ist dieses Ergebnis insofern interessant, als die von Greif ermittelten Wirkfaktoren sich ausschließlich auf die Inhalts-, Methoden- und Intentionskomponente von Coaching-Entscheidungen und nicht auf Coaching-Medien beziehen.

Vor diesem Hintergrund stellt sich die Frage, zu welchen Einschätzungen eine mehr als Prozessforschung angelegte Coaching-Forschung kommen würde (Wastian, 2015; Wegener, 2017).

\subsection{Plausibilitätseinschätzungen und Hypothesen zur Prozess- wirksamkeit eines ausgewählten Coaching-Mediums}

Diese Frage soll im Folgenden exemplarisch für Telefoncoachings diskutiert werden, die mit Unterstützung durch das Coaching-Problemlösungsmedium »Virtuelles Coaching" (VC) (Geißler, 2012) durchgeführt werden. Ziel der Diskussion ist die Entwicklung von Plausibilitätseinschätzungen und Hypothesen, das heißt von empirisch noch zu überprüfenden Vermutungen zur Prozesswirksamkeit derartiger Coachings.

Anhand der in Tabelle 1 dargestellten Komponenten für die Komposition von Coaching-Medien wird erkennbar, dass Telefoncoaching nur zwei der insgesamt sechs Komponenten beinhaltet, nämlich das linguistische und das paralinguistische Kommunikationsverhalten, und dass die Sinnesdaten dieses Verhaltens nichtelektronisch generiert und raumzeitlich elektronisch-synchron vermittelt werden. Gestützt auf empirische Arbeiten zum Telefoncoaching zum einen ohne zusätzliche Coaching-Tools (Berry, 2005; Charbonneau, 2002; Frazee, 2008; Ghods, 2009; McLaughlin, 2013) und zum anderen mit zusätzlicher Unterstützung durch das Problemlösungs-Tool »Virtuelles Coaching « (VC) (Geißler, Hasenbein \& Wegener, 2013; Geißler, Hasenbein, Kanatouri \& Wegener, 2014) sowie auch unter Berücksichtigung empirischer Arbeiten zur telefonischen Psychotherapie (Reese, Conoley \& Brossart, 2002; Simon, Ludman, Tutty, Operkalski \& Von Korff, 2004; Lovell et al., 2006; Ludman, Simon, Tutty \& Von Korff, 2007; Mohr et al., 2012), lassen sich folgende Einschätzungen und Hypothesen zur Wirksamkeit von Telefoncoaching formulieren, das mit 
Unterstützung des Coaching-Problemlösungs-Tools »Virtuelles Coaching « (VC) durchgeführt wird:

- Telefoncoaching macht Coaching ortsunabhängig und bewirkt damit, - dass Reisekosten entfallen und das Coaching damit preiswerter wird,

- dass Klienten sich den Coach ihrer Wahl weltweit suchen können

- und dass Coaches ihr Business weltweit betreiben können.

- Das völlige Fehlen von visuellen Sinnesdaten, die aus dem wechselseitigen Blickkontakt, nonverbalen Körperexpressionen, aus der äußeren Erscheinung der Kommunikationspartner und dem Coaching-Kontext Informationen generieren, bewirkt allem Anschein nach zweierlei:

- zum einen, dass die Bedeutungs- und Wahrnehmungsintensität der uneingeschränkt verfügbaren Sinnesdaten, die sich auf das linguistische und paralinguistische Kommunikationsverhalten beziehen, deutlich gesteigert wird,

- und zum anderen, dass die Gefahr visueller Ablenkung deutlich reduziert wird.

- Die kommunikationstechnisch bedingte Besonderheit, dass in der Telefonkommunikation der räumliche Abstand zwischen der akustischen Erzeugung einer Botschaft durch den Sprecher und seiner Rezeption im Ohr des Hörers sehr viel kleiner ist als in der Face-to-Face-Kommunikation, bewirkt, dass umfangreichere bzw. reichhaltigere Sinnesdaten des paralinguistischen Kommunikationsverhaltens vorliegen. Der Hörer kann zum Beispiel die Atmung und die Klangbildung der von der Sprecherin erzeugten Laute intensiver wahrnehmen und bekommt damit reichhaltigeres Material für die Wahrnehmung von deren Gefühlen und Befindlichkeiten.

- Mit der kommunikationstechnischen Blockierung wechselseitigen Blickkontakts fällt ein zentrales Medium für die wechselseitige Feinsteuerung und Kontrolle des Interaktionsprozesses aus. Das bewirkt

- erstens, dass die Beziehungsgestaltung und Interaktion in der Telefonkommunikation insgesamt fragiler und störungsanfälliger ist (Borlinghaus, o. J.),

- und zweitens, dass diese wichtige Funktion mithilfe der verfügbaren Sinnesdaten des linguistischen und paralinguistischen Kommunikationsverhaltens kompensiert werden muss und entsprechende Kommunikationsfähigkeiten verlangt.

- Auf der anderen Seite beansprucht der wechselseitige Blickkontakt in Verbindung mit nonverbalen Körperexpressionen, vor allem der Mimik in der Face-to-Face-Kommunikation, außerordentliche Aufmerksamkeit, weil die Kommunikationspartner in der Regel ein großes Interesse daran haben, 
beim anderen in einem positiven Licht zu erscheinen. Für Telefoncoaching bedeutet das zweierlei:

- erstens, dass der Druck dieser Aufmerksamkeitsinvestition wegfällt und sehr viel mehr Aufmerksamkeit für die Bearbeitung der vorliegenden Coaching-Thematik zur Verfügung steht (McLaughlin, 2013),

- und zweitens, dass die Klientin einen persönlichen Schutzraum hat, weil der Coach ihr Gesicht und die sich darin spiegelnden Gefühle und Betroffenheiten nicht sehen kann (Geißler et al., 2013; Geißler et al., 2014).

- Weiterhin zeichnet sich Telefoncoaching dadurch aus, dass die Kommunikationspartner ihre äußere Erscheinung, also zum Beispiel die Hautfarbe, ethnische Merkmale der Gesichtszüge, Körperumfang, Frisur und Kleidung sowie den Gesamtzustand hoher oder niedriger körperlicher Gepflegtheit, nicht wahrnehmen können. Das bewirkt zweierlei:

- zum einen, dass eine - allerdings nicht sehr wichtige - diagnostische Erkenntnisquelle wegfällt,

- und zum anderen, dass das Risiko sozialer Vorurteile und persönlicher Übertragungen und Projektionen stark reduziert wird (Clutterbuck, 2010).

- Als Letztes ist zu erwähnen, dass auch das Fehlen aller Sinnesdaten, die Auskunft über den Coaching-Kontext geben, zweierlei bewirkt:

- zum einen, dass die Möglichkeit wegfällt, den Coaching-Kontext so zu gestalten, dass er sich auf die Arbeitsbeziehung positiv auswirkt,

- zum anderen, dass der Klient die Vertrautheit und den Schutz seiner Privatsphäre nicht aufgeben muss, denn der Coach kann zum Beispiel die Unaufgeräumtheit seines Büros oder die vielleicht ärmlich wirkende Ausstattung nicht sehen.

Wird Telefoncoaching durch das E-Coaching-Tool des »Virtuellen Coachings" (VC) angereichert, so wird die demnach zu erwartende Prozesswirksamkeit von Telefoncoaching durch die Merkmale des Tools teilweise bestärkt, teilweise aber auch deutlich modifiziert. Schauen wir zunächst mithilfe des Kategoriensystems von Tabelle 1 auf die Merkmale des Tools, können wir feststellen, dass vorgegebene Texte, und zwar Coaching-Fragen, die teilweise in einer vorgegebenen Sequenz zu bearbeiten sind, und Textfelder für ihre offene Beantwortung, den konzeptionellen Mittelpunkt des Tools bilden und dass die jeweiligen Antworten durch Fotos angereichert werden können, auf denen unterschiedliche Gegenstände und Lebewesen, Menschen und Tiere, 2-D-visuell abgebildet sind. Die Sinnesdaten, die sich auf die vorgegebenen und frei gestaltbaren Texte und die Fotos beziehen, sind alle elektronisch erzeugt und werden im Telefoncoaching 
elektronisch-synchron vermittelt. Damit ergeben sich folgende zu erwartende Prozesswirksamkeiten:

- Die ansonsten im Telefoncoaching bestehende Gefahr visueller Ablenkung besteht nicht, weil die Aufmerksamkeit der Klientin auf die Visualität der Coaching-Fragen und -Antworten und die zusätzlich eingefügten Fotos gerichtet wird.

- Die vorgegebenen Coaching-Fragen und die teilweise vorgegebene Reihenfolge ihrer Bearbeitung gibt dem Coaching-Prozess eine klare, verbindliche Rahmenstruktur und Prozesstransparenz, die die Beziehungsfragilität und -störungsanfälligkeit, unter der Telefoncoaching ansonsten leiden kann, kompensiert.

- Das in das E-Coaching-Tool eingebaute Strukturmerkmal, dass der Klient die Coaching-Fragen, die den Coaching-Prozess anleiten, von vornherein sieht, bewirkt, dass sich seine Aufmerksamkeit nicht nur auf die Bearbeitung der einzelnen Coaching-Fragen richtet, sondern parallel immer auch auf die Gesamtstruktur und die ihr zugrunde liegende Problemlösungsmethode, die damit informell verinnerlicht, das heißt gelernt wird.

- Darüber hinaus ist in das Tool der »sanfte Zwang « eingebaut, dass die Klientin die für sie wichtigen Zwischenergebnisse ihres Dialogs mit dem Coach als Antworten schriftlich fixiert, was bewirkt, dass ihre Eigenständigkeit und Eigenverantwortung gestärkt und die Nachhaltigkeit des Coachings erhöht wird (Held, Korsten \& Reckert, 2012).

- Die Wahl vom Klienten spontan auszuwählender Fotos, mit denen er seine schriftlichen Notizen anreichert, kann dem Coach Einblicke in das Unbewusste des Klienten geben und diesem einen wichtigen zusätzlichen Zugang zur Bearbeitung seiner Problematik eröffnen (Messerschmidt, 2015).

- Diese Aktivitäten der Klientin verlangen viel Aufmerksamkeit und Energie, sodass zu erwarten ist, dass sie nicht durch die visuellen Sinnesdaten wechselseitigen Blickkontakts, nonverbaler Körperexpression und durch die persönliche Erscheinung des Coaches abgelenkt wird.

\section{Ausblick}

Wenn man die eben zusammengestellten Plausibilitätseinschätzungen und Hypothesen betrachtet, lässt sich abschließend feststellen, dass von einem Telefoncoaching, das durch »Virtuelles Coaching " (VC) angereichert wird, insgesamt eine Wirksamkeit zu erwarten ist, welche die Qualität des Coaching-Prozesses mit seinen Sub-Outcomes wie auch den Coaching-Outcome positiv beeinflusst. 
Diese Erwartung ist empirisch durch die Beurteilung von Klienten und Klientinnen bestätigt worden, die dieser Form des Coachings eine hohe Qualität zusprechen, und zwar nicht nur hinsichtlich des Aufbaus einer vertrauensvollen Beziehung zum Coach, sondern auch mit Blick auf das Gefühl emotionaler Sicherheit und Berührtheit, Anregungen zu einem konstruktiven Umgang mit Gefühlen, Selbstreflexion und Möglichkeiten der Thematisierung von psychologisch tief greifenden Aspekten (Geißler et al., 2014).

Auf der anderen Seite darf indes nicht unterschlagen werden, dass viele der oben vorgetragenen Einschätzungen, und zwar vor allem diejenigen zur Wirksamkeit fehlenden Blickkontakts, bisher nur spekulativ sind und dringend empirisch überprüft werden müssen.

\section{Literatur}

Berry, R. M. (2005). A comparison of face-to-face and distance coaching practices: The role of the working alliance in problem resolution. Unpublished doctoral dissertation, Georgia State University, Atlanta, GA.

Blankertz, H. (1969). Theorien und Modelle der Didaktik. München: Juventa.

Borlinghaus, R. (Hrsg.) (o. J.). Coaching 2.0. Kreuzlingen: Bora Text.

Charbonneau, M. A (2002). Participant self-perception about the cause of behavior change from a program of executive coaching. Unpublished doctoral dissertation, Alliant International University, Los Angeles, CA.

Clutterbuck, D. (2010). Welcome to the World of Virtual Coaching and Mentoring. In D. Clutterbuck, Z. Hussain (Eds.), Virtual Coach, Virtual Mentor (pp. 31-52). Charlotte: Information Age Publishing.

Cox, E., Bachkirova, T., Clutterbuck, D. (Eds.) (2010). The Complete Handbook of Coaching. London: Sage.

Frazee, R. V. (2008). E-coaching in organizations. A study of features, practices, and determinants of use. Unpublished doctoral dissertation, San Diego University, CA.

Geißler, H. (2012). Virtuelles Coaching - programmgeleitetes Telefoncoaching mit Internet-Support. In H. Geißler, M. Metz (Hrsg.), E-Coaching und Online-Beratung (S. 137-164). Wiesbaden: Springer VS.

Geißler, H., Hasenbein, M., Kanatouri, S., Wegener, R. (2014). E-coaching: Conceptual and empirical findings of a virtual coaching programme. International Journal of Evidence Based Coaching and Mentoring, 12 (2), 165-187.

Geißler, H., Hasenbein, M., Wegener, R. (2013). E-Coaching. Prozess- und Ergebnisevaluation des »Virtuellen Zielerreichungscoachings«. Organisationsberatung, Supervision, Coaching, 20 (2), 125-142.

Ghods, N. (2009). Distance coaching: The relationship between coach-client relationship, client satisfaction, and coaching outcomes. Unpublished doctoral dissertation, San Diego University, CA.

Grant, A. M. (2006). Workplace and Executive Coaching: A Bibliography from the Scholarly Business Literature. In D. R. Stober, A. M. Grant (Eds.), Evidence Based Coaching Handbook (pp.367-388). Hoboken: John Wiley \& Sons. 
Greif, S. (2015). Allgemeine Wirkfaktoren im Coachingprozesss - Verhaltensbeobachtungen mit einem Ratingverfahren. In H. Geißler, R. Wegener (Hrsg.), Bewertung von Coachingprozessen (S. 51-80). Wiesbaden: Springer.

Greif, S., Schmidt, F., Thamm, A. (2012). Warum und wodurch Coaching wirkt. Ein Überblick zum Stand der Theorieentwicklung und Forschung über Wirkfaktoren. Organisationsberatung, Supervision, Coaching, 19 (4), 375-390.

Held, M., Korsten, H., Reckert, H. (2012). Der Einsatz Virtuellen Transfercoachings in einem Baumarktunternehmen und in einer Bank. In H. Geißler, M. Metz (Hrsg.), E-Coaching und Online-Beratung (S. 209-223). Wiesbaden: Springer VS.

Lovell, K., Cox, D., Haddock, G., Jones, C., Raines, D., Garvey, R., Roberts, C., Hadley, S. (2007). Telephone administered cognitive behaviour therapy for treatment of obsessive compulsive disorder: Randomised controlled non-inferiority trial. The British Medical Journal, 333 (7574), 883.

Ludman, E. J., Simon, G. E., Tutty, S., Von Korff, M. (2007). A randomized trial of telephone psychotherapy and pharmacotherapy for depression: Continuation and durability of effects. Journal of Consulting and Clinical Psychology, 75 (2), 257-266.

McLaughlin, M. (2013). Less is more: The executive coach's experience of working on the telephone. International Journal of Evidence Based Coaching and Mentoring, Special Issue No. 7 (June), 1-13.

Messerschmidt, J. (2015). Das Selbst im Bild. Eine empirische Studie zum Einsatz von Bildmaterialien zur Förderung von Selbstreflexions- und Selbstveränderungsprozessen im Einzelcoaching. Frankfurt a. M.: Lang.

Mohr, D. C., Ho, J., Juffecy, J., Reifler, D., Sokol, L., Burns, M. N., Jin, L., Siddique, J. (2012). Effect of telephone-administered versus face-to-face cognitive behavioural therapy on adherence to therapy and depression outcomes among primary care patients: A randomized trial. The Journal of the American Therapy Association, 307 (21), 2278-2285.

Passmore, J., Peterson, D. B., Freire, T. (Eds.) (2013). The Psychology of Coaching and Mentoring. Oxford: Wiley-Blackwell.

Rauen, C. (2005). Varianten des Coachings im Personalentwicklungsbereich. In C. Rauen (Hrsg.), Handbuch Coaching (3. Aufl., S. 111-136), Göttingen: Hogrefe.

Reese, R. J., Conoley, C. W., Brossart, D. F. (2002). Effectiveness of telephone counselling: A fieldbased investigation. Journal of counselling Psychology, 49 (2), 233-242.

Schreyögg, A. (2012). Coaching (7. Aufl.). Frankfurt a. M.: Campus.

Schulz, W. (1965). Unterricht - Analyse und Planung. In P. Heimann, G. Otto, W. Schulz (Hrsg.), Unterricht - Analyse und Planung (S. 13-47). Hannover: Schroedel.

Simon, G. E., Ludman, E. J., Tutty, S., Operskalski, B., Von Korff, M. (2004). Telephone Psychotherapy and Telephone Care Management for Primary Care Patients Starting Antidepressant Treatment: A Randomized Controlled Trial. The Journal of the American Therapy Association, 292 (8), 935-942.

Wastian, M. (2015). Wirkfaktoren auch im E-Coaching nutzen: Was passiert in der »Black Box» des virtuellen Coaching-Prozesses? In H. Geißler, R. Wegener (Hrsg.), Bewertung von Coachingprozessen (S. 299-321). Wiesbaden: Springer.

Wegener, R. (2017). Bedeutsame Momente im Coaching. Unveröff. Diss., Universität Hamburg. 


\title{
Coaching und die Rolle des Unbewussten
}

\author{
Neurowissenschaftliche Erkenntnisse für \\ eine wirksame Coaching-Praxis
}

Alica Ryba

Der Beitrag zeigt, dass Persönlichkeit und ihre Veränderbarkeit im Coaching eine wichtige Rolle spielen und beides entscheidend durch das Unbewusste beeinflusst wird. Heute wird bereits zwischen Skills, Performance und Development Coaching unterschieden - verschiedene Coaching-Agenden, die den Schwerpunkt entweder auf die Entwicklung von Fähigkeiten, Leistungen oder der Person als Ganzes legen. Es wird die Position vertreten, dass Coaching und Psychotherapie zwei Pole von Beratung mit einem großen Überschneidungsbereich sind. Coaches greifen aktuell vornehmlich auf psychotherapeutische Methoden der zweiten Generation zurück, die nach der Psychoanalyse entwickelt wurden. Damit wird im Coaching einem der wichtigsten Ansätze zur Persönlichkeitsentwicklung, der die Bedeutung des Unbewussten betont, aktuell nur wenig Aufmerksamkeit gewidmet. Der Beitrag beleuchtet die Auffassungen Freuds, des Begründers der Psychoanalyse, und Ericksons, des Begründers moderner Hypnotherapie, zum Unbewussten. Daran anschließend, wird das neurowissenschaftliche Verständnis von Unbewusstem, Vorbewusstem und Bewusstem dargestellt. Dieses fließt ein in das Vier-Ebenen-Modell von Persönlichkeit, das aufzeigt, dass das limbische System als "Sitz« von Psyche und Persönlichkeit einen viel größeren Einfluss auf das Erleben und Verhalten eines Menschen hat als die Großhirnrinde (der Sitz des Bewusstseins). Der Beitrag regt somit an, zwischen zwei unbewusst-limbischen, einer bewusst-limbischen und einer bewussten, kognitiv-sprachlichen Ebene im Gehirn zu unterscheiden, wo sich Veränderungen manifestieren können. Die Autorin spricht sich dafür aus, verschiedene Problemarten und ihre Tiefe differenziert voneinander zu unterscheiden, sodass im Coaching gezielter interveniert werden kann. Eine integrative Vorgehensweise wird empfohlen. 
Ein für Coaching besonders wichtiges, aber noch wenig beachtetes Forschungsergebnis der Neurowissenschaft ist die Erkenntnis, dass menschliches Erleben und Verhalten in erheblichem Umfang durch das Unbewusste vorbereitet und festgelegt wird (vgl. Roth, 2004, S. 59). Lange Zeit galt das bewusste Ich als oberste Kontrollinstanz von Denken, Planen und Handeln. Zunehmend zeigt sich nun, dass das Ich weit weniger Einfluss auf das tatsächliche Verhalten einer Person hat, als diese subjektiv empfindet. Da Coaching auf eine »selbstgesteuerte Erweiterung bzw. Verbesserung der Möglichkeiten des Klienten bzgl. Wahrnehmung, Erleben und Verhalten « abzielt (DBVC, 2016) und diese Aspekte offenbar maßgeblich durch das Unbewusste bestimmt werden, stellt sich die Frage, welche Rolle unbewusste Prozesse im Coaching spielen. Damit beschäftigt sich der folgende Beitrag. Zunächst wird der Stellenwert von Psyche und Persönlichkeit im Coaching beleuchtet, weil dort das Unbewusste letztlich verortet ist. Darauf aufbauend, wird die aktuelle Rolle des Unbewussten in Coaching und Psychotherapie betrachtet. Schließlich werden die neuesten neurowissenschaftlichen Erkenntnisse und ihre Implikationen für das Coaching dargelegt.

\section{Persönlichkeit und Psyche im Coaching}

Coaching hat sich in den vergangenen zwei Jahrzehnten als ein beliebtes Instrument der Personalentwicklung etabliert, das sich weiterhin auf Wachstumskurs befindet und sich immer stärker auf folgenden Ebenen ausdifferenziert (vgl. Roth \& Ryba, 2016, S. 24 ff.):

- Zielgruppen und Praxisfelder (in welchem Kontext findet Coaching statt?);

- bearbeitete Ziele, Anlässe und Themen (was steht auf der Coaching-Agenda?);

- eingesetzte Methoden (welche Coaching-Ansätze werden genutzt?);

- Settings und Art der Coaches (welche Coaching-Varianten werden eingesetzt?).

Seit 2002 sieht sich die Branche mit bedeutenden Fragen der Professionalisierung konfrontiert, die sich durch die zunehmende Diversifizierung nicht leichter beantworten lassen. Sie betreffen die Identität, den Zuständigkeitsbereich, die Qualität und die Wirksamkeit von Coaching. Damit ist auch die Frage berührt, inwiefern die Persönlichkeit des Klienten oder der Klientin und damit das Unbewusste im Coaching-Verständnis abgebildet werden. Die beiden genannten Ebenen »Agenda « und »Ansätze« und die Abgrenzung von Coaching und Psychotherapie sind in diesem Zusammenhang besonders relevant und werden daher im Folgenden näher beleuchtet. 


\subsection{Die Coaching-Agenda}

Auf internationaler Ebene setzt sich langsam die Unterscheidung von mindestens drei verschiedenen Coaching-Agenden durch: Skills, Performance und Development Coaching (vgl. Segers, Vloeberghs \& Hendrickx, 2011; Cox, Bachkirova \& Clutterbuck, 2014). Die drei Agenden umfassen verschiedene inhaltliche Schwerpunkte und bedürfen eines jeweils anderen Zeitaufwands und Engagements. Die Grenzen sind jedoch fließend. Im Skills Coaching steht die Entwicklung von spezifischen, klar abgegrenzten Fähigkeiten im Vordergrund, weshalb ein solches Coaching meist nur wenige Tage oder Wochen beansprucht. Beim Performance Coaching geht es um die Optimierung der beruflichen Leistung und damit häufig um die Verbesserung von Kommunikations-, Interaktions- und Entscheidungsprozessen in komplexen Situationen zum Zweck einer erhöhten Wertschöpfung. Hier ist der Aufwand aufgrund der höheren Komplexität meist umfangreicher, weshalb mehrere Monate für den Coaching-Prozess veranschlagt werden. Beim Development oder auch Life Coaching steht die Person als Ganzes im Fokus; diese Agenda erfordert deshalb das höchste Engagement, es werden mehrere Monate bis Jahre für die Entwicklung eingeplant. Der Coach ist mit intimeren persönlichen und professionellen Fragen konfrontiert. Der Schwerpunkt liegt auf den persönlichen Zielen, Gedanken, Gefühlen und Verhaltensweisen des Individuums und auf der Frage, wie eine Person ihr Leben und ihre Arbeit zu höherer Zufriedenheit und Effektivität verändern kann. In ihrem Buch »Life Coaching in der Praxis« stellen Schmidt-Lellek und Buer (2011) die Hypothese auf, dass hinter beruflichen Themen von Klientinnen oder Klienten häufig Lebensthemen verborgen sind, die im Coaching bearbeitet werden sollten, wenn dieses wirksam sein soll. Spätestens an dieser Stelle wird die heikle Frage der Abgrenzung zwischen Coaching und Psychotherapie offensichtlich.

\subsection{Coaching und Psychotherapie: zwei Pole mit Überschneidungsbereich}

Ob nun im Coaching Fähigkeiten, Leistungen oder die Person als Ganzes entwickelt werden soll - immer geht es auch um Fragen der Persönlichkeit und der Möglichkeiten ihrer Veränderung. Viele Coaches, Personalverantwortliche und Klienten oder Klientinnen befürworten eine klare Trennung zwischen Coaching und Psychotherapie nach dem Motto: Psychotherapie ist ein Heilverfahren für psychisch Kranke, Coaching umfasst hingegen eine Beratung psychisch Gesunder bei beruflichen Fragestellungen (vgl. Price, 2009; Grimmer \& Neukom, 2009); es behandelt also die Wechselwirkungen zwischen Personen und ihrer 
Professions- oder Organisationswelt. In einem Coaching werden weder rein geschäftliche Themen noch schwere psychische Störungen bearbeitet. Die dichotome Gegenüberstellung von Coaching und Therapie stellt sich allerdings bei genauerer Betrachtung als unangemessen heraus; verschiedene Studien (Grimmer \& Neukom, 2009; Maxwell, 2009) legen nahe, dass die Grenze zwischen den beiden Beratungsverfahren fließend ist (für eine ausführliche Darstellung vgl. Roth \& Ryba, 2016). Nicht selten werden im Coaching also Themen wie Burnout, Leistungsabfall aufgrund von Scheidung, hartnäckige Verhaltensmuster, die zum Beispiel Aufstiegsmöglichkeiten verhindern, usw. behandelt. Die Faktoren »Persönlichkeit« und Psyche spielen eine große Rolle, wenn es um das Fühlen, Denken und Handeln von Menschen im beruflichen und privaten Leben geht. Daher liegt der Schluss nahe, dass Coaching und Psychotherapie zwei Pole von Beratung mit einem enormen Überschneidungsbereich sind. An den Coach werden Ansprüche gestellt, die denen einer Psychotherapeutin durchaus entsprechen, was für eine umfassendere Qualifizierung von Coaches spricht.

\subsection{Coaching-Ansätze: theoretische Grundorientierungen von Interventionen}

Methodisch greifen Coaches ohnehin auf eine Fülle von Methoden zurück, die aus verschiedenen therapeutischen Ansätzen stammen, zum Beispiel aus der Verhaltenstherapie oder der Transaktionsanalyse. Jede dieser Denkschulen hat unterschiedliche Konzepte für Persönlichkeitsentwicklung und misst dem Unbewussten eine andere Bedeutung bei. Das Wissen vieler Praktikerinnen und Praktiker über die verschiedenen Ansätze und ihre historischen Wurzeln ist jedoch häufig begrenzt (Roberts \& Jarrett, 2007; Wildflower, 2013).

Vereinfachend, können nach Gilligan (2014) drei Generationen von Ansätzen und Denkschulen unterschieden werden, die sich im Laufe der Zeit entwickelt haben:

- 1. Generation: Traditionelle Psychotherapie

Die traditionelle Psychotherapie wurde erheblich von Sigmund Freud (18561939), dem Begründer der Psychoanalyse, beeinflusst. Ihr Fokus liegt auf Problemen, die als psychische Krankheit aufgefasst werden, und auf der Vergangenheit. Nach dieser Theorie verursachen negative Erfahrungen in der Kindheit aktuelle Probleme. Ziel ist es, durch Einsicht eine Aufarbeitung zu bewirken. Die Bedeutung dieser Denkschule wird darin deutlich, dass die Begriffe »Psychotherapie« und »Psychoanalyse« in der ersten Hälfte des 20. Jahrhunderts meist synonym verwendet wurden (vgl. Kriz, 2007, S. $1 \mathrm{f}$. und 11). 


\section{- 2. Generation: Kurzzeittherapie}

In Abgrenzung zur Pathologisierung und angesichts des immensen Zeitaufwandes einer Psychoanalyse entwickelte sich die zweite Generation von Ansätzen, die Ressourcen, positive Ziele und eine lösungsbezogene Zukunftsfokussierung ins Zentrum stellte. Vor allem diese therapeutischen Ansätze wurden später im Coaching genutzt, das eine Gegenposition zur traditionellen Psychotherapie einnahm. Hier stand nicht Krankheit oder Defizit im Vordergrund, sondern die Unterstützung von gesunden Personen, die ihr berufliches Leben verbessern wollten.

- 3. Generation: Generatives Coaching und andere

$\mathrm{Zu}$ den Ansätzen der dritten Generation zählen solche, die verschiedene Dualitäten in einer Sowohl-als-auch-Haltung integrieren: Probleme/Ressourcen, Vergangenheit/Zukunft, Kognition/Somatik usw. Die meisten Coaches gehen zwar eklektizistisch vor, jedoch fehlt ihnen häufig ein kohärentes, integratives Rahmenmodell.

Die Allgemeine Psychotherapie Klaus Grawes hat auch für das Coaching Vorbildcharakter. Da viele Vertreter von Psychotherapie- und Coaching-Schulen an ihren Denkkonzepten festhalten und sich teilweise vehement bekämpfen, scheint ein sinnvolles Sowohl-als-auch jedoch noch in weiter Ferne.

Nach den Studien von Stahl und Marlinghaus (2000), Whybrow und Palmer (2006) sowie Greif (2014) sind folgende Ansätze heute im Coaching am weitesten verbreitet: systemisch (hauptsächlich in Deutschland), NLP (sowohl in Deutschland als auch in den englischsprachigen Ländern), Transaktionsanalyse (vor allem in Deutschland, aber auch in Großbritannien), lösungsfokussiert (in Deutschland und Großbritannien), kognitiv/verhaltenstherapeutisch (vor allem in Großbritannien, aber auch in Deutschland und den USA). Es wird deutlich, dass psychoanalytische/psychodynamische Ansätze, welche die Bedeutung des Unbewussten stark betonen, im Coaching aktuell eine geringe Rolle spielen. Dabei wird übersehen, dass sich die Ansätze der zweiten Generation nicht nur als Alternative, sondern als Ergänzung zur damals hauptsächlich biografisch verstandenen Beratungsarbeit etabliert haben (Schreyögg, 2013). Im NLP, das sich unter anderem aus der Erickson'schen Hypnose ableitet, wird das Unbewusste berücksichtigt.

Psyche und Persönlichkeit sowie ihre Veränderbarkeit haben also im Coaching durchaus einen wichtigen Stellenwert. Vor diesem Hintergrund beschäftigen wir uns nun konkreter mit dem Unbewussten in Coaching und Psychotherapie. 


\section{Das Unbewusste in Coaching und Psychotherapie}

Der Begriff des »Unbewussten« wurde Anfang des 19. Jahrhunderts im Zusammenhang mit dem animalischen Magnetismus, der Hypnose und der Philosophie der Romantik eingeführt (vgl. Peter, 2009, S. 52). Populär wurde er 1869 durch das Buch »Philosophie des Unbewussten « von Eduard von Hartmann. Dieser systematisierte die romantischen Vorstellungen des Unbewussten und versuchte eine Art Synthese der Ideen Schellings, Spinozas, Hegels, Schopenhauers und anderer. Die psychiatrische Erschließung des Begriffs geht zu einem großen Teil auf Sigmund Freud zurück, der fälschlicherweise auch als »Entdecker des Unbewussten « gilt (Roth \& Strüber, 2014). Im Laufe der Geschichte der Psychotherapie erfuhr der Begriff verschiedene Bedeutungswandlungen, die maßgeblich im Zusammenhang mit Hypnose und Psychoanalyse geprägt wurden. Wir betrachten deshalb nun also kurz die Auffassungen Freuds (Psychoanalyse) und Ericksons (Hypnotherapie), auch wenn es noch weitere Ansätze gibt, in denen das Unbewusste eine Rolle spielt. Daran anschließend, setzen wir uns mit der Relevanz des Unbewussten im Coaching auseinander.

\subsection{Freud und die Lehre vom Unbewussten}

Die Psychoanalyse ist nach Freud die »Lehre vom Unbewussten«. In seinem ersten topischen Modell (griech. topos - der Ort) unterscheidet er im "psychischen Apparat « drei verschiedene seelische Orte bzw. Systeme voneinander: das Unbewusste, das Vorbewusste und das Bewusste (vgl. Freud, 1991, S. 528; Ermann, 2008, S. 35). Freud ging zum einen davon aus, dass die in der Tiefe des Unbewussten lokalisierten Triebwünsche zum Zwecke der Entladung von Triebspannung ihren Weg an die Oberfläche suchen. Zum anderen nahm er an, dass zwischen den drei Systemen Grenzen bestehen, welche die Funktion einer Zensurschranke für die Triebabkömmlinge haben. Aus diesem Grund entstehe ein beträchtliches Maß an Psychodynamik. So würden zum Beispiel Triebabkömmlinge, die eine Bedrohung für das Bewusstsein darstellen könnten, ins Unbewusste verdrängt.

Psychische Störungen werden in diesem Denkmodell als unbewusste Konflikte zwischen den Triebwünschen und ihrer Abwehr verstanden. Diese Kernaussage, die impliziert, dass unbewältigte Erfahrungen der Vergangenheit heutige Konflikte und Störungen verursachen, ist in aktuellen psychodynamischen Modellen erhalten geblieben. Aus neurobiologischer Sicht kann die Aussage im Übrigen bestätigt werden, auch wenn die Trieblehre selbst kritisch zu beurteilen ist (vgl. dazu Roth \& Ryba, 2016, S. 263 und 267). 
In der Konzeption Freuds ist das Unbewusste also ein düsterer Ort, der gefährliche Triebe und das Verdrängte beinhaltet. Daran ist die Vorstellung von primitiven emotionalen und instinktgesteuerten Prozessen gebunden, die eher als bedrohlich betrachtet werden (vgl. Schüßler, 2002, S. 195 f.). Ziel der Psychoanalyse ist es, das Unbewusste durch freie Assoziation und eine sogenannte Übertragungsneurose aufzudecken und damit dessen Macht - in Grenzen - zu brechen. Die auf diese Weise aktualisierten unbewussten Konflikte sollen bewusst und verstehbar werden. Nach Auffassung der Psychoanalyse hat dies eine therapeutische Wirkung, während jedoch aus neurobiologischer Perspektive Einsicht nur einen geringen veränderungsförderlichen Effekt besitzt (vgl. dazu Kapitel 3.2).

\subsection{Das Unbewusste in der Erickson'schen Hypnotherapie}

Der Hypnotherapeut Milton H. Erickson (1901-1980) hat in den 1970er Jahren eine Bedeutungsverschiebung beim Begriff des Unbewussten herbeigeführt; es wurde nun als positive Ressource verstanden (vgl. Peter, 2009, S. 67 f.). Für ihn lag im Unbewussten ein großes Potenzial, und er betonte immer wieder: "your unconscious contains a vast storehouse of learnings, memories and resources" (Erickson, zit. nach Lankton \& Lankton, 2010, S. 18). Ziel der Hypnotherapie ist es, das durch starre Schemata und Denkmuster begrenzte Bewusstsein zu erweitern, indem gezielt unbewusste und unwillkürliche Prozesse angesprochen werden (vgl. Schmidt, 2011, S. 11).

In einer Zeit, in der die Psychoanalyse vorherrschend war, stellte Erickson unter anderem die Glaubenssätze infrage, dass es notwendig sei, Einsicht in unbewusstes Material zu erhalten und vergangene Kindheitserfahrungen zeitintensiv durchzuarbeiten. Stattdessen benutzte er Hypnose, um die bewussten Prozesse der Klientin oder des Klienten außer Kraft zu setzen, sodass dessen Unbewusstes bedeutsame Veränderungen bewirken konnte. Trance wurde von ihm betrachtet als

"eine Periode, in der es dem Patienten gelingt, aus seinen engen Denkrahmen und Glaubenssystemen auszubrechen, so dass er innerlich andere Funktionsmuster erleben kann. Diese anderen Muster sind gewöhnlich Reaktionspotentiale, die durch frühere Lebenserfahrungen gelernt wurden, aber dem Patienten aus dem einen oder anderen Grund unverfügbar bleiben« (Erickson \& Rossi, 2015, S. 14; im Original teilweise kursiv).

Nach diesem Verständnis ermöglicht Trance die Ablösung von rigiden Haltungen und schafft einen bezugsrahmenfreien Zustand der Empfänglichkeit, 
in dem eine Neustrukturierung und Reorganisation möglich wird. Aus neurobiologischer Sicht kann dies bestätigt werden (vgl. Roth \& Ryba, 2016, S. 305).

\subsection{Die Relevanz des Unbewussten im Coaching}

Sowohl psychodynamische als auch hypnotherapeutische Ansätze spielen im heutigen Coaching eine Rolle, wenngleich das Gewicht auf den lösungsorientierten Ansätzen der zweiten Generation liegt. Zu diesen kann das durchaus verbreitete NLP gezählt werden, das stark durch die Hypnotherapie beeinflusst wurde. Insgesamt gibt es nur wenige wissenschaftliche Arbeiten, die sich mit der Rolle des Unbewussten im Coaching auseinandersetzen. Erste integrative Wirkmodelle, die unbewusste Prozesse thematisieren, wurden 2007 von Passmore und 2011 von Rauen, Strehlau und Ubben entwickelt.

Eve Turner veröffentlichte 2010 eine Studie mit dem Titel »Coaches’ views on the relevance of unconscious dynamics to executive coaching «. Mittels eines Online-Fragebogens ( $\mathrm{n}=376)$ und 15 qualitativer Interviews mit Coaches aus Großbritannien ging sie der Frage nach, ob Coaches unbewusste Prozesse im Coaching als relevant einschätzen und ob ihre Ausbildung sie darauf vorbereitet hat, mit solchen Prozessen umzugehen. Darüber hinaus beschäftigt sie sich mit der Frage, ob in der Supervision von Coaches unbewusste Prozesse diskutiert werden und welche Unterschiede Coaches zwischen Coaching und Therapie sehen. 89 Prozent der Coaches gaben im Online-Fragebogen an, dass sie unbewusste Prozesse als relevant im Coaching einschätzen, und 68 Prozent waren der Auffassung, dass solche Prozesse in der Mehrzahl der Dialoge auch vorkommen. Nur drei der befragten Coaches waren der Meinung, dass unbewusste Prozesse im Coaching nicht relevant seien. Eine sehr kleine Anzahl von Coaches sah eine Gefahr in der Auseinandersetzung mit unbewussten Prozessen. Turner (2010, S. 19) schreibt dazu:

"However, some respondents believed coaching should focus on conscious processes as one commented, rather than getting caught up in psychoanalytic depths of the unconscious Coaching deals with the here-and-now and in developing greater conscious awareness to enable increased awareness and capability in relationships and performancer. But this was not typical. The majority believed awareness in this area would support 'people in recognizing hidden or unconscious belief systems . It did not mean unconscious processes always needed to be interpreted.« 
Nur knapp die Hälfte der befragten Coaches (47 \%) gab an, dass ihre Ausbildung sie ausreichend auf den Umgang mit unbewussten Prozessen vorbereitet habe. Am besten vorbereitet fühlten sich Coaches mit langjähriger Erfahrung und einem therapeutischen Hintergrund. 69,9 Prozent gaben im Online-Fragebogen an, dass sie in ihren Supervisionssitzungen unbewusste Prozesse thematisierten. Die interviewten Coaches erklärten, bewusste und unbewusste Prozesse würden sowohl im Coaching als auch in der Therapie beachtet, aber Letztere lege eher den Fokus auf unbewusste Prozesse. Hier wurde allerdings die Einschränkung vorgenommen, dies sei abhängig vom jeweiligen Beratungsansatz. Sowohl im von Turner konzipierten Online-Fragebogen, der nach den von Freud entwickelten Konzepten der Übertragung, Gegenübertragung und Projektion fragt, als auch in den Aussagen der Coaches wird deutlich, dass unbewusste Prozesse aktuell offenbar stark mit einem psychoanalytischen bzw. -psychodynamischen Verständnis in Beziehung gesetzt werden. Dies ist bedauerlich, da es noch viele andere Möglichkeiten gibt, mit dem Unbewussten bzw. Vorbewussten zu arbeiten.

\section{Persönlichkeit und das Unbewusste in den Neurowissenschaften}

In den letzten beiden Jahrzehnten haben die Neurowissenschaften und die Psychologie viele neue Erkenntnisse über die Grundlagen des menschlichen Fühlens, Denkens und Handelns gewonnen. Dazu gehört auch der Befund, dass das Unbewusste das Bewusstsein stärker bestimmt als umgekehrt und dass Letzteres nur in geringem Maße Einsicht in die unbewussten Determinanten des Erlebens und Verhaltens hat, wie ja schon Freud festgestellt hat. Betrachten wir nun das, was aus neurowissenschaftlicher Sicht unter unbewusstem, vorbewusstem und bewusstem Erleben zu verstehen ist, bevor wir uns mit dem Vier-Ebenen-Modell von Psyche und Persönlichkeit auseinandersetzen.

\subsection{Das Unbewusste, das Vorbewusste und das Bewusste}

Tabelle 1 stellt im Überblick das neurowissenschaftliche Verständnis von Unbewusstem, Vorbewusstem und Bewusstem dar (vgl. dazu Roth \& Ryba, 2016). 
Tabelle 1: Das Unbewusste, das Vorbewusste und das Bewusstsein

\begin{tabular}{|c|c|c|}
\hline Das Unbewusste & $\begin{array}{l}\text { Primär unbewusst } \\
\text { Zustände, die grundsätzlich nicht } \\
\text { bewusst gemacht werden kön- } \\
\text { nen, weil sie nicht in einem »be- } \\
\text { wusstseinsfähigen Format» vor- } \\
\text { liegen (z. B. Wahrnehmungen der } \\
\text { sensorischen, corticalen Areale } \\
\text { wie unter anderem die Prozesse } \\
\text { der Netzhaut usw.). } \\
\text { Wahrnehmungsprozesse, welche } \\
\text { die Bewusstseinsschwelle nicht } \\
\text { überschreiten. } \\
\text { Sekundär unbewusst } \\
\text { Wahrnehmungen, die nicht in das } \\
\text { Langzeitgedächtnis gelangen. } \\
\text { Prozesse vor Ausreifung des } \\
\text { Langzeitgedächtnisses (infantile } \\
\text { Amnesie). }\end{array}$ & $\begin{array}{l}\text { Hirnvorgänge, die außer- } \\
\text { halb der Großhirnrinde, also } \\
\text { subcortical ablaufen. Viele } \\
\text { innerhalb der Großhirnrinde } \\
\text { stattfindende Prozesse laufen } \\
\text { auch unbewusst ab. }\end{array}$ \\
\hline $\begin{array}{l}\text { Das Vorbewusste } \\
\text { (Inhalte müssen } \\
\text { vorher immer } \\
\text { bewusst gewesen } \\
\text { sein) }\end{array}$ & $\begin{array}{l}\text { Inhalte des deklarativen Lang- } \\
\text { zeitgedächtnisses (episodisches } \\
\text { Gedächtnis, Wissensgedächtnis } \\
\text { und Bekanntheits- sowie Vertraut- } \\
\text { heitsgedächtnis), die aktuell nicht } \\
\text { bewusst sind, aber bewusst ge- } \\
\text { macht werden können. } \\
\text { Die Inhalte sind je nach Veranke- } \\
\text { rung unterschiedlich leicht bzw. } \\
\text { schwer abrufbar - unter Umstän- } \\
\text { den werden sie ॥sekundär unbe- } \\
\text { wusst», d. h. nicht erinnerbar. } \\
\text { Abläufe, die automatisiert und da- } \\
\text { mit in das prozedurale Langzeit- } \\
\text { gedächtnis abgesunken sind. }\end{array}$ & $\begin{array}{l}\text { Hippocampus = Orga- } \\
\text { nisator des deklarativen } \\
\text { Gedächtnisses. } \\
\text { Langfristiger Speicherort ist } \\
\text { die Großhirnrinde. } \\
\text { Prozedurales } \\
\text { Langzeitgedächtnis: } \\
\text { Basalganglien, Kleinhirn. }\end{array}$ \\
\hline Das Bewusstsein & $\begin{array}{l}\text { Aktualbewusstsein } \\
\text { (Sinneswahrnehmungen; mentale } \\
\text { Zustände; Emotionen, Affekte, } \\
\text { Bedürfniszustände). } \\
\text { Hintergrundbewusstsein } \\
\text { (Identität und Kontinuität; Meinig- } \\
\text { keit des Körpers, Autorschaft und } \\
\text { Kontrollempfinden, Verortung in } \\
\text { Zeit und Raum, Unterscheidung } \\
\text { von Realität und Vorstellung). }\end{array}$ & $\begin{array}{l}\text { Großhirnrinde, insbeson- } \\
\text { dere vorderes und hinteres } \\
\text { Arbeitsgedächtnis. }\end{array}$ \\
\hline
\end{tabular}


Deutlich wird, dass der Begriff des Unbewussten in den Neurowissenschaften anders definiert wird als in psychoanalytischen und hypnotherapeutischen Konzepten. Das, was dort mit dem Unbewussten gemeint ist, entspricht aus neurowissenschaftlicher Perspektive dem tiefen Vorbewussten.

\subsection{Persönlichkeit: rationaler Ratgeber und eigentlicher Akteur}

Hirnphysiologisch betrachtet, wird die Persönlichkeit eines Menschen durch drei Ebenen des limbischen Systems und eine vierte Ebene der Großhirnrinde bestimmt (vgl. zum Folgenden Roth \& Ryba, 2016). Diese Ebenen und die mit ihnen verbundenen Prozesse unterscheiden sich funktional voneinander und sind dem Individuum entweder unbewusst oder vorbewusst/bewusst. Ihre Entwicklung wird durch folgende fünf Faktoren determiniert: Gene im engeren Sinne, die epigenetischen Gen-Regulationsmechanismen, vorgeburtliche Einflüsse, die Bindungserfahrung in den ersten drei Jahren nach der Geburt und den weiteren Sozialisationsprozess.

Die untere limbische Ebene wird vor allem durch genetisch-epigenetische und vorgeburtliche Einflüsse geprägt und ist später nur noch wenig beeinflussbar. Sie sichert über die Kontrolle der vegetativen Funktionen die biologische Existenz und steuert grundlegende affektive Verhaltensweisen wie Angriff, Verteidigung, Flucht, Erstarren, Aggressivität und Sexualverhalten. Außerdem bestimmt sie das Temperament, mit dem ein Mensch geboren wird. Die Prozesse auf dieser Ebene laufen völlig unbewusst ab und können nur indirekt über Erregungen bewusst werden, die in die bewusstseinsfähige Großhirnrinde dringen.

Die mittlere limbische Ebene entwickelt sich vornehmlich in den ersten drei Jahren nach der Geburt und wird wesentlich durch die frühkindliche Bindungserfahrung beeinflusst. Auch wenn diese Zeit teilweise bewusst erlebt wird, sind ihre Inhalte später nicht erinnerungsfähig, weil sich das Langzeitgedächtnis in dieser Phase noch nicht ausgebildet hat. Dies wird als infantile Amnesie bezeichnet. Diese Ebene bildet jedoch die unbewusste Grundlage unserer Kernpersönlichkeit. Sie legt fest, was wir als belohnend empfinden und was uns motiviert. Außerdem bestimmt sie das Verhältnis zu uns selbst und zu anderen und ist die Grundlage unserer Empathiefähigkeit.

Die obere limbische Ebene entwickelt sich etwa ab dem vierten Lebensjahr und ist erst mit 18 bis 20 Jahren einigermaßen »ausgereift«. Sie bettet sozusagen die Kernpersönlichkeit, die durch die beiden unteren Ebenen determiniert ist, in die soziale Welt ein. Auf der Grundlage sozialer Erfahrung bilden sich unser bewusstes Selbst und unser Verständnis von Moral und Ethik heraus. Die Kernpersönlichkeit wird also konkretisiert und passt sich an die Erforder- 
nisse des gesellschaftlichen Lebens an. Dieser Prozess findet bewusst statt. Die obere und die mittlere limbische Ebene können nur durch starke Emotionen verändert werden.

Den drei limbischen Ebenen steht die kognitiv-sprachliche Ebene gegenüber, die vornehmlich in der Großhirnrinde angesiedelt ist. Ihre Entwicklung beginnt ab dem dritten Lebensjahr und verändert sich zeitlebens. Die kognitiv-sprachliche Ebene bildet die Grundlage von Intelligenz, Verstand und Einsicht sowie von planvollem Handeln. Sie ist sozusagen der rationale Ratgeber. Auf dieser Ebene findet auch die rationale oder pseudorationale Darstellung und Rechtfertigung des bewussten Ichs vor sich selbst und anderen statt.

Wichtig ist nun, sich vor Augen zu führen, dass die Großhirnrinde als Sitz von Intelligenz und Verstand keinen direkten Einfluss auf Veränderungen in den limbischen Ebenen hat, die umgekehrt stark auf die kognitiv-sprachliche Ebene einwirken. Die drei limbischen Ebenen sind nämlich der Entstehungsort von Affekten, Gefühlen, Empathie, Motiven, Handlungszielen, Gewissen, Moral und Ethik. Sie prägen somit weitgehend unsere Persönlichkeit und unser egoistisches und soziales Handeln. Dies erklärt, warum kluge Ratschläge und Einsichten allein Menschen meist nicht nachhaltig beeinflussen. Wird nur die kognitive Ebene erreicht, dann wird sozusagen der "rationale Ratgeber «, nicht aber der »eigentliche Akteur« gecoacht. Damit relativiert sich die Wirksamkeit der als zentral eingeschätzten ergebnisorientierten Selbst- und Problemreflexion (Greif, 2008) im Coaching. Dies bedeutet keineswegs, dass Reflexion keine Rolle mehr spielen sollte, sondern dass sie allein nicht alle Problemstellungen lösen kann.

Tabelle 2: Vier-Ebenen-Modell von Psyche und Persönlichkeit

\begin{tabular}{|c|c|c|}
\hline Ebene & Funktionen & Entwicklung \\
\hline $\begin{array}{l}\text { Untere limbische Ebene } \\
\text { Primär unbewusst. } \\
\text { Limbisch-vegetative Grundachse: } \\
\text { Hypothalamus. } \\
\text { Zentrale Amygdala, zentrales Höhlengrau, } \\
\text { vegetative Zentren des Hirnstamms. }\end{array}$ & $\begin{array}{l}\text { Biologische } \\
\text { Funktion } \\
\text { Temperament }\end{array}$ & $\begin{array}{l}\text { Genetische oder } \\
\text { epigenetisch- } \\
\text { vorgeburtliche Ein- } \\
\text { flüsse. Nur wenig } \\
\text { beeinflussbar. }\end{array}$ \\
\hline $\begin{array}{l}\text { Mittlere limbische Ebene } \\
\text { Sekundär unbewusst, d. h. nicht erinne- } \\
\text { rungsfähig aufgrund der infantilen Amnesie. } \\
\text { Kann auch »tief vorbewusste« Anteile } \\
\text { besitzen. } \\
\text { Amygdala. } \\
\text { Mesolimbisches System. }\end{array}$ & $\begin{array}{l}\text { Unbewusste } \\
\text { Grundlage der } \\
\text { Persönlichkeit }\end{array}$ & $\begin{array}{l}\text { Erste drei Lebens- } \\
\text { jahre; frühkindliche } \\
\text { (Bindungs-)Erfahrun- } \\
\text { gen. Nur über starke } \\
\text { emotionale Einwir- } \\
\text { kungen veränderbar. }\end{array}$ \\
\hline
\end{tabular}




\begin{tabular}{|c|c|c|}
\hline Ebene & Funktionen & Entwicklung \\
\hline $\begin{array}{l}\text { Obere limbische Ebene } \\
\text { Bewusst. } \\
\text { Kann ins Vorbewusste absinken. } \\
\text { Limbische Areale der Großhirnrinde: } \\
\text { Prä- und orbitofrontaler, cingulärer und } \\
\text { insulärer Cortex. }\end{array}$ & $\begin{array}{l}\text { Einbettung der } \\
\text { Kernpersönlichkeit } \\
\text { in die soziale Welt }\end{array}$ & $\begin{array}{l}\text { Die Entwicklung } \\
\text { beginnt mit etwa vier } \\
\text { Jahren und ist erst } \\
\text { im Alter von } 18 \text { bis } \\
20 \text { Jahren ausgereift. } \\
\text { Ist nur sozial-emotio- } \\
\text { nal veränderbar. }\end{array}$ \\
\hline $\begin{array}{l}\text { Kognitiv-sprachliche Ebene } \\
\text { Bewusst. } \\
\text { Kann ins Vorbewusste absinken. } \\
\text { Linke Großhirnrinde, besonders die } \\
\text { Sprachzentren und der präfrontale Cortex, } \\
\text { cortico-hippocampales System. }\end{array}$ & $\begin{array}{l}\text { Rationaler Rat- } \\
\text { geber (Ratgeber } \\
\text { ist nicht Akteur; } \\
\text { hat nur geringen } \\
\text { Einfluss!) }\end{array}$ & $\begin{array}{l}\text { Die Entwicklung } \\
\text { beginnt mit etwa drei } \\
\text { Jahren und verändert } \\
\text { sich ein Leben lang. }\end{array}$ \\
\hline
\end{tabular}

\section{Was bedeutet dies nun für wirksames Coaching?}

Die bisherigen Ausführungen haben zwei Punkte deutlich gemacht. Erstens spielen Psyche und Persönlichkeit sowie ihre Veränderbarkeit auch im Coaching eine große Rolle. Zweitens wird die Persönlichkeit eines Menschen entscheidend durch unbewusste und vorbewusste Inhalte und Prozesse auf den limbischen Ebenen bestimmt. Es ist deutlich geworden, dass der Einfluss der kognitiven Ebene auf das Erleben und Verhalten recht gering ausfällt, sodass tief greifende Veränderung nur durch starke emotionale Einwirkung auf den drei limbischen Ebenen erreicht werden kann. Hier spielen das Unbewusste und das Vorbewusste eine bedeutende Rolle. Das heute häufig stark kognitiv-reflexionsorientierte Coaching sollte daher hinterfragt und basierend auf diesen Erkenntnissen weiterentwickelt werden.

Im Coaching geht es nicht immer um tief greifende Persönlichkeitsentwicklung. Manchmal kann für die Klientin oder den Klienten ein Fortschritt bereits darin bestehen, durch Reflexion eine neue Perspektive auf ein Thema zu gewinnen oder eigene Fähigkeiten weiterzuentwickeln. Aus diesem Grund ist es, vor allem im Coaching, notwendig, Problemarten, -ursachen und -tiefen präzise voneinander zu unterscheiden. Roth und Ryba (2016) bieten dafür die in Tabelle 3 dargestellten, unspezifischen - im Sinne von grundlegenden - und spezifischen Ansatzpunkte für Coaching und Therapie an und ordnen diese dem Vier-Ebenen-Modell der Persönlichkeit zu. 
Tabelle 3: Unspezifische und spezifische Ansatzpunkte für Coaching und Therapie (in Anlehnung an Roth \& Ryba, 2016, S. 353 f.)

\begin{tabular}{|c|c|c|}
\hline Ansatzpunkt & Erläuterung & $\begin{array}{l}\text { Neurowissen- } \\
\text { schaftliche } \\
\text { Ebene }\end{array}$ \\
\hline \multicolumn{3}{|c|}{ Unspezifische Ansatzpunkte } \\
\hline Zielklärung & $\begin{array}{l}\text { Konkrete und genaue Beschreibung von } \\
\text { Zielen und möglichen Problemlösungen. }\end{array}$ & KE, OLE \\
\hline Ressourcenaktivierung & $\begin{array}{l}\text { Hinweis auf Stärken des Klienten/der Patien- } \\
\text { tin und die Möglichkeit, sich in dieser Weise } \\
\text { zu erleben und zu verhalten. }\end{array}$ & KE, OLE \\
\hline $\begin{array}{l}\text { Problemaktualisierung } \\
\text { und Erlebnisaktivierung }\end{array}$ & $\begin{array}{l}\text { Aktivierung der neuronalen Netzwerke, die } \\
\text { am Problem beteiligt sind. }\end{array}$ & KE, OLE, MLE \\
\hline \multicolumn{3}{|l|}{ Spezifische Ansatzpunkte } \\
\hline $\begin{array}{l}\text { Umgehung des } \\
\text { Problems }\end{array}$ & $\begin{array}{l}\text { Vermeidung des Problems oder Veränderung } \\
\text { von Rahmenbedingungen dergestalt, dass } \\
\text { das Problem nicht mehr auftritt. }\end{array}$ & OLE, MLE \\
\hline $\begin{array}{l}\text { Symptom- oder } \\
\text { Problembeseitigung }\end{array}$ & $\begin{array}{l}\text { Beseitigung eines Symptoms oder Problems, } \\
\text { das im Laufe der Zeit seine ursprüngliche } \\
\text { Funktion verloren hat. }\end{array}$ & OLE, MLE \\
\hline $\begin{array}{l}\text { Kognitiv-motivationaler } \\
\text { Perspektivwechsel }\end{array}$ & $\begin{array}{l}\text { Vermittlung der Einsicht in die Hintergründe } \\
\text { des Erlebens und Verhaltens durch ergebnis- } \\
\text { orientierte Selbst- und Problemreflexion. }\end{array}$ & KE, OLE \\
\hline $\begin{array}{l}\text { Emotionen und } \\
\text { Körperempfindungen }\end{array}$ & $\begin{array}{l}\text { Vergegenwärtigung und Nacherleben von } \\
\text { Emotionen sowie Abschwächung und Selbst- } \\
\text { beruhigung von überschießenden Affekten. } \\
\text { Überwindung einer »neuromuskulären } \\
\text { Blockade». }\end{array}$ & $\begin{array}{l}\text { OLE, MLE, } \\
\text { ggf. ULE }\end{array}$ \\
\hline $\begin{array}{l}\text { Prozedurales Einüben } \\
\text { besser angepasster } \\
\text { Verhaltensweisen }\end{array}$ & $\begin{array}{l}\text { Vollzieht sich bei leichteren Belastungen } \\
\text { in corticalen limbischen, bei schwereren, } \\
\text { "strukturellen« Defiziten in subcorticalen } \\
\text { limbischen Strukturen. Neu eingeübte Verhal- } \\
\text { tensweisen löschen nicht die vorhandenen, } \\
\text { sondern überdecken sie. }\end{array}$ & OLE, MLE \\
\hline $\begin{array}{l}\text { Aufbau von neuen Fä- } \\
\text { higkeiten und wichtige } \\
\text { Erfahrungen }\end{array}$ & $\begin{array}{l}\text { Systematische Weiterentwicklung »un- } \\
\text { reifer» Fähigkeiten, Nachholen wichtiger } \\
\text { Erfahrungen. }\end{array}$ & OLE, MLE \\
\hline $\begin{array}{l}\text { Umstrukturierung der } \\
\text { inneren Landkarte }\end{array}$ & $\begin{array}{l}\text { Verlassen des bisherigen, zu engen Bezugs- } \\
\text { rahmens und Neuorganisation der inneren } \\
\text { Landkarte. Eine Reihe von Untersuchungen } \\
\text { zeigt, dass das Umlernen und Neu-Erleben } \\
\text { unter Hypnose erleichtert zu sein scheint. }\end{array}$ & KE, OLE, MLE \\
\hline $\begin{array}{l}\text { Entscheidungs- und } \\
\text { Loslassprozesse }\end{array}$ & $\begin{array}{l}\text { Trennung von dysfunktional gewordenen } \\
\text { Vorstellungen und Fantasien. }\end{array}$ & KE, OLE \\
\hline
\end{tabular}




\begin{tabular}{|l|l|l|}
\hline Ansatzpunkt & Erläuterung & $\begin{array}{l}\text { Neurowissen- } \\
\text { schaftliche } \\
\text { Ebene }\end{array}$ \\
\hline $\begin{array}{l}\text { Umsetzungsunterstüt- } \\
\text { zung und Evaluation }\end{array}$ & $\begin{array}{l}\text { Förderung der Umsetzung der Ziele im All- } \\
\text { tag, Aktions- oder Zeitpläne, Durchdenken } \\
\text { von Realisierungsmöglichkeiten, Antizipation } \\
\text { möglicher Hindernisse, Einplanen von Beloh- } \\
\text { nungen usw. Kontrolle des Erfolgs der Inter- } \\
\text { ventionen und gegebenenfalls Nachbessern. }\end{array}$ & KE, OLE, MLE \\
\hline
\end{tabular}

Abkürzungen: $\mathrm{KE}$ = bewusste, kognitiv-sprachliche Ebene; OLE = bewusste obere limbische Ebene; MLE = nicht erinnerungsfähige bzw. tief vorbewusste, mittlere limbische Ebene; $\mathrm{ULE}=$ unbewusste, untere limbische Ebene

Grob kann unterschieden werden zwischen eher »oberflächlichen «, das heißt weniger tief liegenden Problemen, und hartnäckigen Erlebens- und Verhaltensmustern, denen meist emotionale Konflikte im Sinne des psychodynamischen Ansatzes zugrunde liegen. Um bei Letzteren professionell wirksam sein zu können, braucht es auch im ergebnisorientierten Coaching Kenntnisse in Psychodynamik. Eine unkritische und unreflektierte Übernahme der psychodynamischen Therapiekonzepte ist jedoch nicht zu empfehlen. Aus heutiger Sicht sind einige von Freuds Annahmen nicht haltbar (vgl. für eine ausführliche Darstellung Roth \& Ryba, 2016). Zum anderen ist eine Methodik, die auf Einsicht setzt, aus neurowissenschaftlicher Sicht wenig geeignet, um das limbische System als Sitz von Psyche und Persönlichkeit nachhaltig zu verändern. Hier bietet es sich eher an, mit hypnotherapeutischen Methoden zu arbeiten, da im Zustand der Trance ein besserer Zugriff auf vorbewusste Inhalte und eine Neustrukturierung von internalen Netzwerken möglich ist.

Insgesamt wäre es wünschenswert, im Sinne von Gilligan und Dilts eine Sowohl-als-auch-Haltung im Coaching zu etablieren. Auf diese Weise könnten die Stärken der jeweiligen Coaching-Ansätze sinnvoll in einem kohärenten Coaching-Modell integriert werden, das idealerweise neurowissenschaftlich fundiert ist. 


\section{Literatur}

Cox, E., Bachkirova, T., Clutterbuck, D. (2014). The Complete Handbook of Coaching ( $2^{\text {nd }}$ ed.). Los Angeles: Sage.

DBVC (2016). Definition Coaching. www.dbvc.de/der-verband/ueber-uns/definition-coaching. html [15.2.2017].

Erickson, M. H., Rossi, E. L. (2015). Hypnotherapie. Aufbau - Beispiele - Forschungen. Stuttgart: Klett-Cotta.

Ermann, M. (2008). Freud und die Psychoanalyse: Entdeckungen, Entwicklungen, Perspektiven. Stuttgart: Kohlhammer.

Freud, S. (1991). Die Traumdeutung. Frankfurt a. M.: Fischer.

Gilligan, S. (2014). Generative Coaching - kreatives Bewusstsein nutzen. In A. Ryba, D. Pauw, D. Ginati, S. Rietmann (Hrsg.), Professionell coachen. Das Methodenbuch: Erfahrungswissen und Interventionstechniken von 50 Coachingexperten (S. 390-400). Weinheim: Beltz.

Greif, S. (2008). Coaching und ergebnisorientierte Selbstreflexion. Göttingen: Hogrefe.

Greif, S. (2014). Coaching und Wissenschaft - Geschichte einer schwierigen Beziehung. Organisationsberatung, Supervision, Coaching, 21 (3), 295-311.

Grimmer, B. Neukom, M. (2009). Coaching und Psychotherapie. Gemeinsamkeiten und Unterschiede - Abgrenzung oder Integration? Wiesbaden: VS Verlag für Sozialwissenschaften.

Kriz, J. (2007). Grundkonzepte der Psychotherapie (6., vollst. überarb. Aufl.). Weinheim: Beltz.

Lankton, S. R., Lankton, C. H. (2010). The Answer Within. A Clinical Framework of Ericksonian Hypnotherapy. Bethel: Crown House.

Maxwell, A. (2009). How do business coaches experience the boundary between coaching and therapy/counselling? Coaching: An International Journal of Theory, Research and Practice, 2 (2), 149-162.

Passmore, J. (2007). An Integrative Model for Executive Coaching. Consulting Psychology Journal: Practice and Research, 59 (1), 68-78.

Peter, B. (2009). Die Ideengeschichte des Unbewussten in Hypnose und Psychoanalyse. HypnoseZHH. 4 (1+2), 49-78.

Price, J. (2009). The coaching/therapy boundary in organizational coaching. Coaching: An International Journal of Theory, Research and Practice, 2 (2), 135-148.

Rauen, C., Strehlau, A., Ubben, M. (2011). Eine integrative Theorie über die grundlegenden Wirkzusammenhänge im Coaching. In B. Birgmeier (Hrsg.), Coachingwissen (S. 147-160). Wiesbaden: VS Verlag für Sozialwissenschaften.

Roberts, V. Z., Jarrett, M. (2007). What is the difference and what makes the difference? A comparative study of psychodynamic approaches to executive coaching. In H. Brunning (Ed.), Executive Coaching: Systems-Psychodynamic Perspective (pp. 3-39). London: Karnac Books.

Roth, G. (2004). Das Verhältnis von bewusster und unbewusster Verhaltenssteuerung. Psychotherapie Forum, 12 (2), 59-70.

Roth, G., Ryba, A. (2016). Coaching, Beratung und Gehirn. Neurobiologische Grundlagen wirksamer Veränderungskonzepte. Stuttgart: Klett-Cotta.

Roth, G., Strüber, N. (2014). Wie das Gehirn die Seele macht. Stuttgart: Klett-Cotta.

Schmidt, G. (2011). Einführung in die hypnosystemische Therapie und Beratung (4. Aufl.). Heidelberg: Carl-Auer.

Schmidt-Lellek, C. J., Buer, F. (2011). Life-Coaching in der Praxis - Wie Coaches umfassend beraten. Göttingen: Vandenhoeck \& Ruprecht.

Schreyögg, A. (2013). Psychodynamische Perspektiven im Coaching und in der Organisationsberatung. Organisationsberatung, Supervision, Coaching, 20 (4), 375-377. 
Schüßler, G. (2002). Aktuelle Konzeption des Unbewussten - Empirische Ergebnisse der Neurobiologie, Kognitionswissenschaften, Sozialpsychologie und Emotionsforschung. Zeitschrift für Psychosomatische Medizin und Psychotherapie, 48 (2), 192-214.

Segers, J., Vloeberghs, D., Hendrickx, E. (2011). Structuring and Understanding the Coaching Industry: The Coaching Cube. Academy of Management Learning \& Education, 10 (2), 204-221.

Stahl, G. K., Marlinghaus, R. (2000). Coaching von Führungskräften: Anlässe, Methoden, Erfolg Ergebnisse einer Befragung von Coaches und Personalverantwortlichen, Zeitschrift für Führung und Organisation, 69 (4), 199-207.

Turner, E. (2010). Coaches' views on the relevance of unconscious dynamics to executive coaching. Coaching: An International Journal of Theory, Research and Practice, 3 (1), 12-29.

Whybrow, A., Palmer, S. (2006). Taking stock: A survey of Coaching Psychologists' practices and perspectives. International Coaching Psychology Review, 1 (1), 56-70.

Wildflower, L. (2013). The Hidden History of Coaching. UK: McGraw-Hill Education. 


\title{
Das Selbst im Bild
}

\section{Mit Bildern Selbstreflexion und Veränderung im Coaching wirksam unterstützen}

\author{
Jasmin Messerschmidt
}

\begin{abstract}
Die Promotionsstudie, die hier vorgestellt wird, untersuchte die Wirkungen, die mit der Betrachtung von Bildmaterialien wie Fotos oder Postkarten im Einzelcoaching erzielt werden. Im Mittelpunkt des Forschungsinteresses standen Prozesse des Zugangs zu unbewussten Anteilen der Persönlichkeit, der ergebnisorientierten Selbstreflexion und der Einfluss dieser Methode auf die Coach-Klienten-Beziehung. In dialogorientierten, leitfadengestützten Experteninterviews berichteten acht Coaches und vier ihrer Klientinnen und Klienten über ihre Erfahrungen mit dieser Interventionsform. Dieser Beitrag stellt die Promotionsstudie in den aktuellen theoretischen Kontext, beschreibt das methodische Vorgehen und beleuchtet zentrale Ergebnisse der Studie und deren Bedeutung für die Coaching-Forschung und -Praxis.
\end{abstract}

In meiner empirischen Forschungsarbeit ging ich der Fragestellung nach, inwieweit über den Einsatz und die Betrachtung von konkreten Bildmaterialien wie Fotos, Magazinbilder oder Postkarten im Einzelcoaching wirksame Veränderungsprozesse unterstützt werden können. Mein Forschungsinteresse gründete auf drei Aspekten. Zum einen hatte ich als langjährig praktizierende Coach häufig beeindruckende Erfahrungen mit dieser Interventionsform gemacht und interessierte mich persönlich für deren Wirkmechanismen. Zum anderen folgte ich den Anregungen von Fachexperten, Prozesse der Aktivierung unbewusster Persönlichkeitsanteile, der ergebnisorientierten Selbstreflexion und die Coach-Klienten-7Beziehung wissenschaftlich zu untersuchen und relevante Wirkfaktoren herauszuarbeiten (vgl. Rauen, Strehlau \& Ubben, 2011, S. 159). Drittens lagen zu Beginn der Studie zum Einsatz von Bildmaterialien erst wenige Fachartikel oder empirische Untersuchungen vor, mit Ausnahme des $\mathrm{ZRM}^{\circledR}$-Ansatzes von Storch und Krause (2005). Absicht war es, mit einer empirischen Promotionsstudie diese Forschungslücke zu schließen und zum Erkenntnisgewinn und zur Theoriebildung beizutragen. 
Der Blick auf die relevante aktuelle Theorie zeigt, dass ein professionelles Coaching Entwicklungs- und Veränderungsprozesse über die systematische Förderung von Selbstreflexion und Selbstwahrnehmung unterstützt (vgl. Dietz, Holetz \& Schreyögg, 2010, S. 18). Nach Greif (vgl. 2008, S. 22) setzt erfolgreiche Selbstreflexion eine gelungene Selbstexploration voraus, bei der unbewusste Anteile des Selbstbildes der bewussten Reflexion zugänglich gemacht und verbalisiert werden können. Dieser Prozess gelingt über die Aktivierung des unbewussten Selbstsystems (vgl. Rauen et al., 2011, S. 159), über das der Klient einen Eindruck von seinen unbewussten Bedürfnissen, Motiven und Interessen erhält (vgl. Rauen et al., 2011, S. 155 f.). Befunde der Neurowissenschaften belegen die Annahme von zwei grundsätzlich unterschiedlich funktionierenden Gedächtnissystemen, dem »expliziten « (bewusst) und dem »impliziten« (unbewusst) Gedächtnis (vgl. Grawe, 2004, S. 74). Diese Unterscheidung gilt nicht nur für Gedächtnisprozesse, »sondern auch für Wahrnehmungen, Lernen, Emotionen, Handlungssteuerung, Motivation, Emotionsregulation und das Beziehungsverhalten « (ebd., S. 124). Gemeinsam steuern und erzeugen beide Gedächtnissysteme menschliches Verhalten und Erleben, wobei nach aktuellen neurowissenschaftlichen Erkenntnissen davon auszugehen ist, dass die meisten Prozesse unbewusst verlaufen (vgl. Grawe, 2000, S. 235).

Um im Coaching wirksame Entwicklungs- und Selbstreflexionsprozesse anzuregen, sollten sowohl bewusste als auch unbewusste Bereiche der Persönlichkeit einbezogen werden (vgl. Grawe, 2004, S. 435). Experten (vgl. Hüther, Roth, Storch \& Krause, ref. nach Messerschmidt, 2015, S. 126) vertreten die Meinung, dass eine nachhaltige Verhaltensänderung mehr verlangt als einen rein bewussten, verbalen Austausch. Da sprachliche Methoden überwiegend mit dem expliziten Gedächtnissystem verknüpft sind, sprechen sie implizite Prozesse nur wenig an. Grawe (2000, S. 240) konstatiert: »Darüber reden gibt keinen Zugriff auf diese (unbewussten) Prozesse. « Auch nach Ansicht von Storch und Krause (2005, S. 45) bewirkt ein rein bewusster, verbaler Austausch lediglich ein »intellektuelles Geplänkel im Kopf, das typischerweise nicht zum Vollzug von Handlungen führt «. Die Schwierigkeit besteht jedoch darin, dass implizite Vorgänge weder willentlich aktiviert, abgerufen, beeinflusst noch sprachlich ausgedrückt werden können (vgl. Grawe, 2000, S. 240). Hierzu merkt auch Roth an: »Wir sind uns selber undurchdringlich. Das (bewusste) Ich kann sich nicht oder nicht gründlich durchschauen!« (G. Roth, 2008, S. 277).

Für ein wirkungsvolles Coaching stellt sich damit die Frage, wie unbewusste Aspekte in den Coaching-Prozess einbezogen werden können, um effektive und kongruente, das heißt sowohl für bewusste als auch für unbewusste Persönlichkeitsanteile stimmige Selbstveränderungsprozesse zu initiieren. Experten 
wie Kuhl und Strehlau (2011, S. 179) sowie Rauen et al. (2011, S. 158) empfehlen den Einsatz von nonverbalen, bildhaften und kreativen Methoden, da diese das Anregen, Bewusstwerden und Bearbeiten von impliziten Aspekten ermöglichen. Insbesondere Bilder können explizite und implizite Gedächtnisprozesse miteinander verbinden und die Kommunikation mit dem eigenen Unbewussten unterstützen (Bucci, ref. nach Storch, 2011, S. 131).

Der Zugang zu unbewussten Prozessen erfolgt bei bildhaften Methoden über einen Projektionsvorgang. Bilder können Resonanzen auslösen, indem sie unbewusste Erfahrungen im Selbstsystem von Menschen ansprechen (vgl. Greif, 2008, S. 116). Über diese Projektion werden Bilder zu Symbolen, Trägern von impliziten Botschaften (W. Roth, 2011, S. 160). Es öffnet sich der Zugang zu unbewussten Anteilen, wodurch der Dialog zwischen bewussten und unbewussten Ebenen gefördert wird (vgl. Greif, 2008, S. 116). In der Arbeit mit Bildmaterialien stellt sich für den Klienten oder die Klientin im Coaching daher die Frage: »Was hat die Resonanz, die das Bild in mir ausgelöst, mit mir zu tun?«

\section{Methodisches Vorgehen}

Im Fokus der Studie standen die Erfahrungen, die Coaches und deren Klientinnen oder Klienten mit dem Einsatz von Bildmaterialien wie Fotos oder Postkarten im Coaching gesammelt hatten. Entlang der aktuellen Theorie wurden vier zentrale Forschungsfragen aufgeworfen (vgl. Messerschmidt, 2015, S. 203 ff.).

Die erste Fragestellung untersuchte aus der Klienten- und Coach-Perspektive, welche Wirkungen mit der Betrachtung von Bildmaterialien im Coaching einhergehen. Dabei lag der Fokus auf Aspekten, die mit der Aktivierung von unbewussten und Selbstreflexions-Prozessen im Zusammenhang stehen. Zweitens wurde der Frage nachgegangen, wie der Einsatz von Bildmaterialien im Coaching methodisch professionell gestaltet werden kann, um Veränderungsabsichten von Klientinnen und Klienten bestmöglich zu unterstützen. Hier interessierte, inwieweit sich aus den Daten Gesetzmäßigkeiten für den Einsatz von Bildmaterialien im Einzelcoaching ableiten ließen und damit ein Beitrag zur Praxeologie von Coaching geleistet werden konnte. Drittens wurde erforscht, welche formalen und inhaltlichen Anforderungen Bildmaterialien erfüllen sollten. Angesichts der Relevanz einer vertrauensvollen Zusammenarbeit wurde in einer vierten Fragestellung der Einfluss dieser Interventionsform auf die CoachKlienten-Beziehung untersucht.

Um das subjektive Erleben von Personen, die mit dieser Methode Erfahrung gesammelt hatten, angemessen nachvollziehen und verstehen zu können, wurde 
ein qualitativer, verstehender Ansatz gewählt (vgl. ebd., S. 206). Es wurden Experteninterviews mit Coaches und Klientinnen oder Klienten durchgeführt, bei der die Befragten über die subjektiven Erfahrungen, die sie mit dem Einsatz von Bildmaterialien im Einzelcoaching gesammelt hatten, Auskunft gaben. Die Interviews mit den Coaches fokussierten auf deren professionelles Selbstverständnis, ihr methodisches Vorgehen, ihr eigenes subjektives Erleben sowie auf Hypothesen und Beobachtungen, wie ihre Klientinnen und Klienten den Einsatz von Bildmaterialien im Einzelcoaching erleben und was er bei ihnen bewirkt. Aus der Klientenperspektive interessierten vor allem die individuellen Erfahrungen und Wirkungen dieser Interventionsform im Hinblick auf die individuelle Zielsetzung. Da die Befragten auch über implizite Prozesse berichteten, wurde ein offener, beschreibender und interpretierender Ansatz gewählt.

Die individuellen Erfahrungen und Einschätzungen der befragten Expertinnen und Experten wurden mit leitfadengestützten Experteninterviews erhoben. Es wurden je ein Leitfaden für Coaches und ein zweiter für Klientinnen und Klienten entwickelt. Die Konzeption der beiden Leitfäden erfolgte theoriegeleitet. Die Fragen des Leitfadens waren offen formuliert, da unbewusste Erfahrungen nicht direkt abgefragt werden können, sondern erschlossen werden müssen (vgl. Meuser \& Nagel, 1994, ref. nach Bogner \& Menz, 2005, S. 42). Über dieses Instrument war es zudem möglich, sich flexibel an den jeweiligen Interviewpartner anzupassen und auch spontan neue, in der Konzeption der Leitfäden bislang unberücksichtigte Aspekte zu vertiefen und darüber zu einer Theorieerweiterung beizutragen.

Insgesamt wurden zwölf Experteninterviews mit acht in dieser Interventionsform erfahrenen Coaches und vier ihrer Klienten und Klientinnen durchgeführt. Die Auswertung der Daten erfolgte mit der qualitativen Inhaltsanalyse nach Mayring. Zunächst wurde auf der Basis des Datenmaterials und des theoretisch konzipierten Leitfadens ein Kategoriensystem entwickelt, anhand dessen die transkribierten Interviews entlang der Forschungsfragen ausgewertet und interpretiert wurden. Überwiegend wurden die Kategorien deduktiv, das heißt theoriegeleitet, an das Textmaterial herangetragen. Einige Kategorien konnten induktiv aus dem Textmaterial gewonnen werden. Insgesamt wurden 55 Kategorien gebildet, die zu 10 Hauptkategorien ${ }^{1}$ zusammengefasst wurden (vgl. Messerschmidt, 2015, S. 227).

1 Die Hauptkategorien lauteten: »Einsatzbereiche von Bildmaterialien im Coaching «, »Qualitäten von Bildmaterialien im Vergleich zu Sprache«, »Bilder als Medium«, »Einsatz von Bildmaterialien zur Unterstützung von Selbstreflexionsprozessen im Coaching«, »Bilder als Brücke zwischen Coach und Klient«, »Qualitäten von Bildern «, »Situative Passung zum Einsatz der Methode«, »Methodisches Vorgehen«, »Professionelles Selbstverständnis und persönlicher Bezug zur Methode«, »Bewertung der Methode«. 


\section{Forschungsergebnisse}

Im Folgenden werden die wichtigsten Ergebnisse entlang der vier zentralen Forschungsfragen aus beiden Perspektiven dargelegt. ${ }^{2}$ Zum besseren Verständnis skizziere ich zunächst das Vorgehen der befragten Coaches in der Arbeit mit Bildmaterialien, bevor ich auf die Wirkungen dieser Interventionsform eingehe. Anschließend werden die Anforderungen an Bildmaterialien und die Auswirkungen dieser Interventionsform auf die Coach-Klienten-Beziehung dargestellt.

\section{Erste Forschungsfrage: Wie gehen Coaches methodisch vor?}

Die acht befragten Coaches arbeiten entweder mit selbst zusammengestellten Postkartensammlungen, mit Fotos oder mit der "ZRM ${ }^{\circledR}$-Bildkartei« von Krause und Storch (2010). Der Prozessablauf besteht aus den Prozessschritten »Ankündigung der Methode«, »Auslage der Bildmaterialien« durch den Coach, »Formulieren einer Fragestellung» (z. B. »Welches Bild würde Sie bei Ihrem Anliegen unterstützen?«), der »Bildauswahl durch den Klienten«, dem »Prozess der Bedeutungsgebung « und dem »Abschluss«. In der Arbeit mit Bildmaterialien nehmen die Coaches die Rolle eines Prozessgestalters ein und erachten den Klienten als kompetenten Experten seines Lebens und seiner Entwicklung. Im Prozess passen die Coaches die Methode situativ an ihre Klientinnen und Klienten und deren Anliegen an und wechseln zwischen drei verschiedenen Ebenen: einer emotional-empathischen Ebene, einer analytischen Beobachterebene und einer metakognitiven Prozesssteuerungsebene. In der zentralen Phase der Bedeutungsgebung gestalten die Coaches einen »entspannten « Dialog, der die Kommunikation zwischen unbewussten und bewussten Anteilen anregen soll. Klient und Coach stehen miteinander in einer intensiven Verbindung und arbeiten die tiefere Bedeutung des Bildes für den Klienten oder die Klientin heraus mit dem Ziel, über das Bild Klarheit und Erkenntnisse über sich selbst als Person zu gewinnen. Dabei halten sich die Coaches mit eigenen Deutungen zurück (vgl. Messerschmidt, 2015, S. 369 ff.). Als wichtig wird erachtet, dem Klienten oder der Klientin das Bild im Anschluss an das Coaching mitzugeben, da es (zu) ihm gehöre (vgl. ebd., S. 298).

2 Originalzitate der interviewten Coaches und Klienten sind in Anführungszeichen gesetzt. 


\section{Zweite Forschungsfrage: Welche Wirkungen wurden durch die Betrachtung von Bildmaterialien erzielt?}

Sowohl Coaches als auch Klientinnen oder Klienten brachten zum Ausdruck, dass über Bilder im Vergleich zu sprachlichen Methoden neue Qualitäten in Coaching-Prozesse eingebracht würden. Nach Aussage der Coaches bewirken Bilder bei Klienten und Klientinnen »ein anderes Denken«, sie »erweitern Perspektiven und lassen Veränderbarkeit erkennen «, ermöglichen »Konkretisierung und Klarheit«. Klientenaussagen bestätigten diese Feststellungen und stellten vor allem den Gewinn von »Klarheit« heraus (vgl. ebd., S. 361).

Bestätigt wurde die emotionsfördernde Wirkung von Bildmaterialien. Bilder »laden« Klientinnen und Klienten in ihre Gefühlswelt »ein«, womit emotionale Aspekte in den Coaching-Prozess integriert werden können, was nach Ansicht von Hirnforschern auch notwendig ist, um bedeutsame Veränderungen zu erreichen (Hüther, ref. nach Hüther, Tatschl \& Walther, 2010, S. 68). Zudem erleichtern Bilder das Sprechen über Gefühle und tragen zu einer positiven Stimmung im Coaching-Prozess bei. Die beiden letztgenannten Aspekte erweitern die theoretischen Vorannahmen zur Wirkung von Bildmaterialien und können daher als Erkenntnisgewinn der Studie gewertet werden (vgl. Messerschmidt, 2015, S. 362). Gleichzeitig erlauben Bilder Klientinnen und Klienten auch, in Distanz zu ihren Gefühlen zu gehen und diese aus einer Metaperspektive zu betrachten. Dies lässt den Schluss zu, dass der Einsatz von Bildmaterialien generell bei emotionalen Anliegen - und insbesondere bei Themen, bei denen negative Emotionen verarbeitet werden müssen - Entwicklungsprozesse wirksam unterstützt (vgl. ebd.).

Im Hinblick auf den Zugang zu unbewussten Prozessen beschrieben die Befragten, dass Klienten und Klientinnen Bilder nach dem Kriterium aussuchten, inwieweit sie sich in einem Bild wiederfänden, sich damit identifizierten oder in Resonanz gehen würden. Beide Befragtengruppen berichteten von einer starken emotionalen Verbindung des Klienten oder der Klientin mit seinem/ihrem Bild. Es zeigte sich, dass Bilder wie eine »Brücke« oder ein »Medium« wirken können, über die der Klient, die Klientin in einen intensiven Kontakt mit sich selbst tritt und Zugang zu unbewussten Anteilen erhält. Bei hoher Identifikation lösen Bilder intensive Denk- und Fühlprozesse aus. Klientinnen und Klienten denken intensiv über sich nach und erforschen sich im Dialog mit dem Bild selbst. Das Bild wird für sie zum »Spiegel« ihres Selbst und berührt sie tief (vgl. ebd., S. 363). Bislang unbewusste Ziele, Bedürfnisse, zentrale Lebensthemen und verschüttete Ressourcen können erkannt, in Worte gefasst und in den Coaching-Prozess integriert werden. Bei diffusen Anliegen kann frühzeitig der tat- 
sächliche Beratungsbedarf erkannt werden, was die Effizienz und Effektivität von Coaching-Prozessen steigert. Insgesamt stärken die Ergebnisse die Annahme von Forschern, nach der über kreative Verfahren der Zugang zu unbewussten Prozessen unterstützt wird (vgl. ebd., S. 364).

Ein wesentliches Interesse der Studie galt der Erforschung der selbstreflexionsfördernden Wirkung von Bildmaterialien. Der durch die Bilder angeregte Dialog zwischen bewussten und unbewussten Aspekten bildet hierfür die Basis, da nach Ansicht von Experten die Komplexität von Selbstreflexionsprozessen nur durch die Integration von abwechselnd stattfindenden emotionalen und rationalen bzw. bewussten und impliziten Prozessen bewältigt werden kann (Kuhl, 2001, ref. nach Offermanns, 2004, S. 105). Wie beschrieben, lösen Bilder bei Klientinnen und Klienten Denkprozesse über sich selbst als Person und über das eigene Denken, Fühlen und Handeln aus. Dieses intensive Erforschen von unbewussten Aspekten des Selbst kann als Selbstexploration im Rahmen von Selbstreflexionsprozessen bezeichnet werden. Bei den Befragten führte es zu neuen bewussten Erkenntnissen und Einsichten über sich selbst. Darüber hinaus vergleichen Klienten und Klientinnen die reale Ist-Situation, in der sie sich befinden, mit der auf dem Bild repräsentierten idealen Soll-Situation. Über diesen Vergleich werden weitere Selbstreflexionsprozesse angeregt, was bei negativer Diskrepanz zu konkreten Veränderungsabsichten führt. Nach Auswertung des Datenmaterials konnte die theoretisch angenommene selbstreflexionsfördernde Wirkung von Bildmaterialien eindeutig bestätigt werden (vgl. Messerschmidt, 2015, S. 365).

In ihrer abschließenden Bewertung äußerten sich die befragten Klientinnen und Klienten wertschätzend, anerkennend und zufrieden mit dem Einsatz von Bildmaterialien im Coaching und beschrieben sie als eine angenehme Methode, mit der »effektive« Ergebnisse mit »Leichtigkeit« erzielt würden und selbst tief gehende Themen in einer "entspannten Atmosphäre " mit "Spaß« mühelos bearbeitet werden könnten. Bilder weckten positive Emotionen und ermöglichten, schnell und einfach »Klarheit« zu gewinnen. Aus Klientensicht hebt sich diese Interventionsform positiv von sprachlichen Methoden ab und stellt einen deutlichen Mehrwert dar. Auch die befragten Coaches erachteten Bilder insgesamt als eine »starke « und "gut funktionierende « Intervention und als »Bereicherung «. Bilder bewirkten »neue Erkenntnisse« und »Klarheit« und könnten tiefer liegende Prozesse »gut in Gang « bringen. Es handle sich um ein vielseitig einsetzbares Medium, das im Coaching von einer sanften Erinnerungshilfe bis hin zu emotionalem Erleben eingesetzt werden könne. Coaches schätzen, dass der Einsatz von Bildern von positiven Emotionen begleitet werde und die Intervention mit Bildern »Freude« bzw. »Spaß« mache. Es gebe keine Hin- 
weise für negative Erfahrungen. Mit Blick auf die nachhaltige Wirksamkeit der Methode äußerten sich die Coaches spekulativ und schätzten diese heterogen und teilweise widersprüchlich ein (vgl. ebd., S. 375 f.).

Nach Ansicht der Coaches passt die Arbeit mit Bildern gut zu Klienten und Klientinnen, die Zugang zu ihren Gefühlen haben und eine Offenheit für ungewöhnliche Methoden zeigen. Auf die Ankündigung der Methode reagierten Klientinnen und Klienten meist »überrascht « oder »irritiert«, wobei sich diese Erstreaktion sehr schnell in »Zuspruch« bzw. »Begeisterung« umwandle. Für einen erfolgreichen Methodeneinsatz solle der Coach die Relevanz unbewusster Prozesse für das menschliche Erleben und Handeln anerkennen, er solle von der Wirkung dieser Methode überzeugt sein und sie situativ passend zu Klientin und Anliegen einsetzen können (vgl. ebd., S. 369). Zudem sollte er Kunden und Kundinnen ein authentisches und professionelles Erklärungsmodell anbieten können, wie beispielsweise das Konzept der somatischen Marker von Damasio, die Multiple Code Theory von Bucci oder die auf C. G. Jung zurückgehende Annahme der Resonanzbildung, um Akzeptanz für eine »ungewöhnliche Methode« erzielen zu können (vgl. ebd., S. 373).

Als mögliche Gefahr wurde von beiden Befragtengruppen thematisiert, dass aufgrund der emotionsfördernden, stark öffnenden Wirkung in der Arbeit mit Bildmaterialien der Coaching-Rahmen leicht verlassen werden könne. Ein Coach müsse bei der Anwendung dieser »mächtigen« Methode reflektiert und professionell vorgehen. Als wichtige Voraussetzungen für einen erfolgreichen Methodeneinsatz wurden eine fundierte Beraterausbildung, eigene Erfahrungen und die Auseinandersetzung mit der Methode sowie ein reflektiertes Bewusstsein über die potenziell starken Wirkungen genannt (vgl. ebd., S. 376f.).

\section{Dritte Forschungsfrage: Welche Bildmaterialien eignen sich?}

Als wesentliche inhaltliche Anforderungen an Bildmaterialien wurden die Aspekte »Assoziationsfreiraum « und »Ausdrucksstärke« herausgearbeitet. Bilder mit einem großen Assoziationsfreiraum lösten bei unterschiedlichen Menschen verschiedenste Assoziationen und Gefühle aus und seien "mehrdeutig « interpretierbar. Ausdrucksstarke Bilder sprächen Emotionen stärker an. Die Ausdrucksstärke eines Bildes sei von der farblichen Gestaltung, dem Motiv und der Bildgröße abhängig. Coaches und Klienten betonten, Bildmotive dürften keine Gefühle von Angst oder Kontrollverlust auslösen. Zudem sollten eigene Sammlungen von Bildmaterialien vielseitig sein, das heißt sowohl abstrakte, konkrete, schwarz-weiße als auch farbige Bilder enthalten und eine professionelle, seriöse Wirkung bei Klientinnen und Klienten erzielen (vgl. ebd., S. 367 f.). 


\section{Vierte Forschungsfrage: Welchen Einfluss haben Bilder auf die Coach-Klienten-Beziehung?}

Nach Aussagen der Coaches wirken sich Bilder positiv auf die Beziehungsqualität aus. Sie förderten als »Türöffner« oder »Brücke« den Kontakt und die »Verknüpfung « zwischen Coach und Klient auf eine angenehme, unkomplizierte Art und würden sie einladen, sich auf einer gemeinsamen emotionalen Ebene zu begegnen. Dies mache sich insbesondere in schwierigen Konstellationen oder in Anfangssituationen positiv bemerkbar. Allerdings besitzt die Wahl der eingesetzten Interventionsform für beide Expertengruppen, Coaches wie Klienten oder Klientinnen, eine eher nachrangige Bedeutung. Insbesondere Klientinnen und Klienten betonten, dass die Qualität der gemeinsamen Arbeit vom Vertrauen in den Coach und in seine Kompetenzen bestimmt werde (vgl. ebd., S. 366).

\section{Fazit und Ausblick}

Die Studie bestätigt, dass Bilder bei Klienten und Klientinnen wirksam den Zugang zu sich, zu Emotionen und unbewussten Prozessen unterstützen und als »Medium « die intrapersonale Kommunikation zwischen bewussten und unbewussten Bereichen der Persönlichkeit fördern. Es werden intensive Selbstreflexionsprozesse angestoßen, in denen sich Klientinnen und Klienten bislang unbewusster Bedürfnisse, Gefühle und Lebensthemen gewahr werden und über die Auseinandersetzung mit dem Bild neue bewusste Erkenntnisse und Einsichten über sich als Person gewinnen. Dieser erweiterte Blick eröffnet den Zugang zu verschütteten Ressourcen und ermöglicht es, kreative Lösungen und alternative Handlungsoptionen zu entwickeln.

Angesichts der Bedeutung impliziter Prozesse für das menschliche Erleben und Handeln zeigt sich die Relevanz der Methode im Führungskräfte-Coaching: Der Einsatz von Bildmaterialien unterstützt, dass bislang unbewusste Bedürfnisse, Gefühle, Motive zu einem bewussten Teil der Person werden, mit der sich die Führungskraft auseinandersetzen und die sie in ihr bewusstes Ich integrieren kann. Stimmige Entwicklungsziele können identifiziert und bearbeitet werden, wodurch ein ganzheitlicher Veränderungsprozess unterstützt wird, der sich im Sinne von Jung auch als »Bewusstwerdungsprozess« bezeichnen lässt. Dies zeigt, dass der Einsatz von Bildern im Coaching die Entwicklung einer stimmigen (Führungs-)Persönlichkeit unterstützt, die mit den Herausforderungen der Umwelt wirksam umgehen kann (vgl. ebd., S. 379). 
Gleichzeitig stellt sich aufgrund der tiefen Wirkung dieser Intervention die Frage nach der therapeutischen Grenze im Business-Kontext. Die positive Wirkung von Bildern hängt eindeutig davon ab, dass der Coach verantwortungsbewusst, reflektiert und professionell im Rahmen seines beraterischen Könnens handelt. Zudem sollten die eingesetzten Bilder ausschließlich positive Assoziationen hervorrufen sowie ausdrucksstark und vielfältig interpretierbar sein. Die Studie verweist darauf, dass die Methode zum Coach passen muss, er muss ferner dem Kunden oder der Kundin ein plausibles Erklärungsmodell für deren Einsatz anbieten können. Deutlich wird auch der Bedarf nach Anschlussstudien, um valide und eindeutige Aussagen über die nachhaltige Wirksamkeit dieser Interventionsform treffen zu können.

Ein Blick in die Zukunft einer zunehmend globalisierten Arbeitswelt macht deutlich, dass Bildverfahren sehr gut in virtuellen Coachings eingesetzt werden könnten.

Die herausragende Bedeutung einer vertrauensvollen Coach-Klienten-Beziehung für ein erfolgreiches Coaching wird durch die Studie erneut bestätigt. Es hat sich einmal mehr gezeigt, dass das Vertrauen in den Coach als wichtiger erachtet wird als der Einsatz einer spezifischen Methode.

Als Fazit kann festgehalten werden, dass der Einsatz von Bildern eine vielseitig verwendbare Interventionsform ist, die menschliche Entwicklungsprozesse wirkungsvoll unterstützt, eine neue Qualität in den Prozess einbringt und dass sie von Klientinnen oder Klienten und Coaches aufgrund ihrer Wirkungen gleichermaßen geschätzt wird.

\section{Literatur}

Bogner, A., Menz, W. (2005). Das theoriegenerierende Experteninterview - Erkenntnisinteresse, Wissensformen, Interaktion. In A. Bogner, B. Littig, W. Menz (Hrsg.), Das Experteninterview - Theorie, Methode, Anwendung (2. Aufl., S. 33-70). Wiesbaden: VS Verlag für Sozialwissenschaften.

Dietz, T., Holetz, K., Schreyögg, A. (2010). Begriffsbestimmung. In Deutscher Bundesverband Coaching e. V. (Hrsg.), Leitlinien und Empfehlungen für die Entwicklung von Coaching als Profession (3., erw. Aufl., S. 18-19). Wetzlar: wd print + medien.

Grawe, K. (2000). Psychologische Therapie (2., korr. Aufl.). Göttingen: Hogrefe.

Grawe. K. (2004). Neuropsychotherapie. Göttingen: Hogrefe.

Greif, S. (2008). Coaching und ergebnisorientierte Selbstreflexion. Göttingen: Hogrefe.

Hüther, G., Tatschl, S., Walther I. (2010). Das Gehirn wird so, wie und wofür man es mit Begeisterung nutzt. In W. Knopf, I. Walther (Hrsg.), Beratung mit Hirn - Neurowissenschaftliche Erkenntnisse für die Praxis von Supervision und Coaching (S. 62-71). Wien: Facultas.

Krause, F., Storch, M. (2010). Ressourcen aktivieren mit dem Unbewussten. Bern: Huber. 
Kuhl, J., Strehlau, A. (2011) Handlungspsychologische Grundlagen des Coaching. In B. Birgmeier (Hrsg.), Coachingwissen - Denn sie wissen nicht, was sie tun? (2., akt. u. erw. Aufl., S. 173-184). Wiesbaden: VS Verlag für Sozialwissenschaften.

Mayring, P. (2003). Qualitative Inhaltsanalyse - Grundlage und Techniken (8. Aufl.). Weinheim: Beltz.

Messerschmidt, J. (2015). Das Selbst im Bild - Eine empirische Studie zum Einsatz von Bildmaterialien zur Förderung von Selbstreflexions- und Selbstveränderungsprozessen im Einzelcoaching. Frankfurt a. M.: Lang.

Offermanns, M. (2004). Braucht Coaching einen Coach? Eine evaluative Pilotstudie. Stuttgart: ibidem.

Rauen, C., Strehlau, A., Ubben, M. (2011). Eine integrative Theorie über die grundlegenden Wirkzusammenhänge im Coaching. In B. Birgmeier (Hrsg.), Coachingwissen - Denn sie wissen nicht, was sie tun? (2., akt. u. erw. Aufl., S. 147-160). Wiesbaden: VS Verlag für Sozialwissenschaften.

Roth, G. (2008). Persönlichkeit, Entscheidung und Verhalten - warum es so schwierig ist, sich und andere $z u$ ändern (4. Aufl.). Stuttgart: Klett-Cotta.

Roth, W. (2011). C. G. Jung verstehen (2. Aufl. der Neuausg. v. 2009). Ostfildern: Patmos.

Storch, M. (2011). Embodiment im Zürcher Ressoucenmodell (ZRM). In M. Storch, B. Cantieni, G. Hüther, W. Tschacher, Embodiment. Die Wechselwirkung von Körper und Psyche verstehen und nutzen (2., erw. Aufl., S. 127-142). Bern: Huber.

Storch, M., Krause, F. (2005). Selbstmanagement - ressourcenorientiert. Grundlagen und Trainingsmanual für die Arbeit mit dem Zürcher Ressourcen Modell ZRM (3., korr. Aufl.). Bern: Huber. 


\title{
Prozessbezogene Determinanten der Wirkung von Einzelcoaching
}

\author{
Ein systematischer Überblick über den \\ internationalen Forschungsstand
}

Cornelia Tonhäuser

Vor dem Hintergrund des zuweilen als defizitär gekennzeichneten Forschungsstands bezüglich Wirkungen und Wirkfaktoren von Coaching widmet sich der Beitrag der Frage, welche Faktoren auf der Ebene des Coaching-Prozesses die Wirkung von Einzelcoaching fördern oder hemmen. Anhand einer umfassenden Analyse der empirischen Forschungsliteratur wird ein Überblick über die internationale Forschung zu den prozessbezogenen Determinanten der Wirkung von Einzelcoaching gegeben. Die Ergebnisse werden differenziert nach Determinanten der Person des Coaches, der Arbeitsbeziehung sowie des Coaching-Prozesses. Insgesamt konnten acht empirisch bestätigte prozessbezogene Wirkfaktoren identifiziert und in ihren Facetten näher spezifiziert werden. Die Analyse verdeutlicht, dass die bisherige quantitativ-empirische Forschung auf klare Tendenzen im Hinblick auf Faktoren mit positivem Einfluss auf die Wirkung bzw. den Erfolg von Coaching auf der Ebene der Coaching-Maßnahmen verweist. In der Tat zeigen sich bei den Befunden zu den erfolgsrelevanten Determinanten auf der Ebene des Coaching-Prozesses insgesamt nur wenige Inhomogenitäten. Abschließend werden Empfehlungen für die zukünftige Forschung gegeben.

\section{Ausgangslage}

Unstrittig ist, dass Coaching ein wirksames Angebot zur Beratung und Unterstützung von Personen und Personalentwicklungsprozessen in Unternehmen ist. Künzli (2005, S. 240) bringt es prägnant auf den Punkt: »Coaching wirkt, und die Wirkungen sind teilweise beträchtlich!« Aber die Wirksamkeitsforschung im Kontext von Coaching befindet sich noch in den Anfängen, sodass sowohl 
aus wissenschaftlicher als auch aus praktischer Perspektive noch erhebliche Unsicherheiten über die zentralen Wirkfaktoren im Coaching bestehen. Dieser Beitrag widmet sich den prozessbezogenen Wirkfaktoren, das heißt den Einflussfaktoren auf der Ebene des Coaching-Prozesses. Wirkfaktoren werden hier verstanden als empirisch bestätigte Determinanten, welche die Wirkung bzw. den Erfolg eines Coaching-Prozesses positiv oder negativ beeinflussen (Wechsler, 2012). Um Aufschluss über die prozessbezogenen Wirkfaktoren im Einzelcoaching zu erhalten, werden aufgrund einer Analyse des internationalen Forschungsstands die empirisch bestätigten erfolgsrelevanten Determinanten auf der Ebene des Coaching-Prozesses systematisiert.

\section{Methodisches Vorgehen}

Die Identifizierung und Prüfung empirischer Studien im Hinblick auf zentrale prozessbezogene Determinanten der Coaching-Wirkung basiert methodisch auf einer Analyse von relevanten Literaturdatenbanken. Die Literaturrecherche erfolgte anhand von zentralen deutsch- und englischsprachigen Schlüsselwörtern in folgenden Online-Datenbanken: PSYNDEX, PsycINFO, FIS Bildung und Business Source Complete. Die Analyse umfasst die Zeitspanne von 2004 bis 2015. Frühere Publikationen sind bereits von Künzli (2005) dargestellt worden. Mithilfe der ersten drei Datenbanken konnten vor allem empirische Arbeiten im Kontext der pädagogisch-psychologischen Wirksamkeitsforschung identifiziert werden. Die letztgenannte Datenbank hat es ermöglicht, vorrangig empirische Studien aus dem Bereich der betriebswissenschaftlich orientierten Wirksamkeitsforschung im Kontext von Coaching zu eruieren.

In einem zweiten Schritt wurden Kriterien für den Einbezug von Studien aus der Datenbankrecherche definiert. Einbezogen wurden nur quantitative Studien zum Einzelcoaching, die Wirk- bzw. Erfolgsfaktoren auf der Ebene des Coaching-Prozesses untersuchen. Um eine möglichst hohe Qualität der in die Analyse einbezogenen empirischen Befunde zu gewährleisten, wurden ausschließlich quantitativ-empirische Untersuchungen berücksichtigt, die statistisch signifikante Zusammenhänge sowie Effektstärken meist auf der Grundlage von Regressions-, Strukturgleichungs- oder Varianzanalysen berichten. Studien, die nicht signifikante Ergebnisse berichten, wurden nicht berücksichtigt, da ihre Befunde statistisch nicht gegen Zufall abgesichert sind. Nicht berücksichtigt wurden des Weiteren Studien, die primär die Ergebnis- bzw. Produktebene betrachten, Arbeiten, die auf Wirkungen von anderen Coaching-Formen als Einzelcoaching fokussieren, sowie qualitative Studien. Der gewählte 
Forschungsrahmen ermöglicht somit einen umfassenden Blick auf den aktuellen internationalen Forschungsstand zu den empirisch bestätigten erfolgsrelevanten Determinanten auf der Ebene des Coaching-Prozesses.

\section{3 Überblick über die prozessbezogenen Determinanten}

Insgesamt sind 24 Studien durch die Datenbankrecherche identifiziert worden, die den zuvor festgelegten Kriterien entsprechen. Darunter befindet sich eine Metaanalyse von Sonesh et al. (2015). Differenziert nach den Forschungsdesigns, weisen lediglich zwei der einbezogenen Studien ein experimentelles Design mit randomisierter Versuchs- und Kontrollgruppe auf. Dieses Vorgehen besitzt die höchste interne Validität und entspricht hohen wissenschaftlichen Qualitätsstandards, da kausale Wirkbeziehungen erschlossen werden (Künzli \& Stulz, 2009). Sieben Studien haben ein quasiexperimentelles Design mit einer Versuchs- und Kontrollgruppe, aber ohne randomisierte Zuteilung der Probanden. Bei diesem Design ist die interne Validität gefährdet, da alternative Ergebniserklärungen und Störvariablen nicht ausgeschlossen werden. Die Mehrzahl von 14 Studien weist ein nichtexperimentelles Design auf. Es handelt sich um Vorher-Nachher-Studien ohne Kontrollgruppen oder um Querschnittsstudien. Da bei nichtexperimentellen Designs keine Vergleichsmöglichkeiten zu Ausgangswerten oder Kontrollgruppen gegeben sind und Störvariablen nicht ausgeschlossen werden, ist die wissenschaftliche Aussagekraft der Befunde aufgrund der nicht gegebenen internen Validität eingeschränkt. Obwohl das Gros der einbezogenen Studien methodische Limitationen aufweist, verweisen sie dennoch auf deutliche Tendenzen und leisten einen wesentlichen Betrag zur notwendigen empirischen Fundierung von Coaching, wie nachfolgend dargelegt wird. Insgesamt konnten im Rahmen der Analyse des Forschungsstandes acht statistisch signifikante Determinanten der Wirkung von Einzelcoaching auf der Ebene des Coaching-Prozesses identifiziert werden. Des Weiteren sind zahlreiche Facetten im Sinne von Teilaspekten dieser Determinanten systematisiert worden. Die in der Analyse der Forschungsliteratur identifizierten erfolgsrelevanten Determinanten auf der Ebene des Coaching-Prozesses werden nachfolgend differenziert dargestellt nach:

- Merkmalen des Coaches,

- Merkmalen der Arbeitsbeziehung zwischen Coach und Klient/-in sowie

- Merkmalen des Coaching-Prozesses. 


\subsection{Merkmale des Coaches}

$\mathrm{Zu}$ den in der bisherigen empirischen Forschung identifizierten statistisch signifikanten Determinanten des Coaches gehören dessen Involvement und Commitment, die beruflichen Kompetenzen und Qualifikationen sowie das Kommunikations- und Führungsverhalten. Zum Einfluss soziodemografischer Variablen des Coaches, wie beispielsweise Alter, Geschlecht, Umfang der Erfahrungen als Coach in Berufsjahren oder Ausbildungshintergrund, liegen bislang keinerlei (auch keine nicht signifikanten) empirischen Ergebnisse vor, sodass hier weiter erheblicher Forschungsbedarf besteht.

Bezüglich der Merkmale des Coaches konnten bislang fünf statistisch signifikante Faktoren mit positivem Einfluss auf die Coaching-Wirkung empirisch bestätigt werden. Die vorliegenden Befunde sind diesbezüglich homogen und zeigen somit einheitliche Tendenzen auf. So bestätigt die Studie von Schmidt und Keil (2004) den positiven Einfluss der Determinante "Involvement des Coaches«. Involvement meint hier das Engagement des Coaches, dessen Motivation und Fähigkeit, Vertrauen und Akzeptanz herzustellen, sowie dessen Interesse am Klienten, an der Klientin. Des Weiteren bestätigen zwei Studien (Boyce, Jackson \& Neal, 2010; Gan \& Chong, 2015) den signifikant positiven Einfluss der Determinante »Commitment des Coaches« gegenüber den Klientinnen und Klienten sowie dem Unternehmen. Zwei Studien (Jansen, Mäthner \& Bachmann, 2004; Offermanns, 2004) verweisen zudem auf die positiven Effekte der vorhandenen Kompetenzen und Qualifikationen des Coaches auf die Coaching-Wirkung. Als Facetten dieser Determinante bestätigen zwei Studien (Schmidt \& Keil, 2004; Bozer, Sarros \& Santora, 2014) die fachliche Kompetenz und Glaubwürdigkeit des Coaches. Bozer et al. (2014) konnten ferner den positiven Einfluss der psychologischen Kenntnisse des Coaches als Facette dieser Determinante bestätigen. Als Einflussfaktor erweist sich auch die Kommunikationskompetenz des Coaches, die in mehreren Studien in ihren verschiedenen Facetten empirisch gut untersucht wurde. Die vorliegenden Studien indizieren unisono einen signifikant positiven Einfluss der Facetten »Beherrschen von Fragetechniken « (Jansen et al., 2004), »aktives Zuhören « (Jansen et al., 2004; de Haan, Culpin \& Curd, 2011) sowie »Feedback geben « (Jansen et al., 2004; Offermanns, 2004; Kappenberg, 2008; Bozer et al., 2014; Ladegard \& Gjerde, 2014; MacKie, 2014). Das Führungsverhalten des Coaches im Coaching-Prozess erweist sich ebenfalls als erfolgsrelevantes Merkmal. Als Facette des Führungsverhaltens konnte sowohl in der Studie von Gatling (2014) als auch in der Metaanalyse von Sonesh et al. (2015) der positive Einfluss eines authentischen Verhaltens des Coaches bestätigt werden, das näher charakterisiert wird 
durch Selbstbewusstsein, ausgewogene Verarbeitung, relationale Transparenz und internalisierte moralische Perspektive. Sonesh et al. (2015) zeigen auf, dass ein authentischer Coach einen positiven Einfluss auf den Informationsaustausch zwischen Coach und Coachee hat. Die Studie von Mühlberger und Traut-Mattausch (2015) verweist auf den signifikant positiven Einfluss der Aspekte »transaktionales" und "transformationales Führungsverhalten « seitens des Coaches. Ladegard und Gjerde (2014) bestätigen den positiven Effekt von facilitativem Verhalten des Coaches als Form transformationaler Führung. Interessant sind auch zwei Studien (Ianiro \& Kauffeld, 2012; Ianiro, Lehmann-Willenbrock \& Kauffeld, 2015), die den positiven Einfluss eines dominant-freundlichen Verhaltens des Coaches bestätigen.

\subsection{Merkmale der Arbeitsbeziehung}

Auf der Ebene der Arbeitsbeziehung zwischen Coach und Klient/-in lassen sich in den Studien zwei Wirkfaktoren identifizieren: die Qualität der Beziehung zwischen Coach und Klient/-in und die Stärke des Arbeitsbündnisses. Für die Determinante der Beziehungsqualität besteht recht gute empirische Evidenz. Mehrere Studien konnten übereinstimmend einen signifikant positiven Einfluss auf die Coaching-Wirkung bestätigen (z. B. Offermanns, 2004; Jansen et al., 2004; de Haan \& Mannhardt, 2013). Empathie, Wertschätzung und emotionale Unterstützung seitens des Coaches gegenüber dem Klienten oder der Klientin sind zentrale empirisch bestätigte Facetten der Beziehungsqualität (Schmidt \& Keil, 2004; Jansen et al., 2004; Runde, Bastians \& Weiss, 2005; Behrendt, 2006; de Haan et al., 2011). Eng damit assoziiert ist als weitere Facette das gegenseitige Vertrauen in der Arbeitsbeziehung (Schmidt \& Keil, 2004; Jansen et al., 2004; Kappenberg, 2008; Boyce et al., 2010). Uneinheitlich sind hingegen die Befunde zum Persönlichkeitsmatching. Dies meint gute Passung und hohe Ähnlichkeit zwischen Coach und Klient/-in in Bezug auf Einstellungen, Werte, Überzeugungen, Arbeitsstile sowie Geschlecht (Bozer, Joo \& Santora, 2015). Während die Studie von Bozer et al. (2015) bei diesem Aspekt einen signifikant positiven Effekt auf die Coaching-Wirkung aufzeigt, konnten Boyce et al. (2010) keinen Einfluss bestätigen.

Auf der Ebene der Arbeitsbeziehung erweist sich ein tragfähiges Arbeitsbündnis als weitere prozessbezogene Determinante der Coaching-Wirkung. Als Facetten, die ein tragfähiges Arbeitsbündnis kennzeichnen, wurden bislang vier Aspekte empirisch bestätigt. So konnten eine Metaanalyse (Sonesh et al., 2015) und drei weitere Studien (Baron \& Morin, 2009; de Haan et al., 2011; de Haan, Duckworth, Birch \& Jones, 2013) den signifikant positiven Ein- 
fluss der »Working Alliance« (Bordin, 1979) nachweisen. Dieses ursprünglich aus der Therapieforschung stammende Konzept meint den Aufbau eines starken Arbeitsbündnisses, das auf Kollaboration, Commitment und Übereinstimmung zwischen Coach und Klient/-in über die Art der Zusammenarbeit beruht. Des Weiteren lässt sich Working Alliance spezifizieren durch die Verbindung (bond), die sich zwischen Coach und Coachee entwickelt, die Ziele (goals), die mit der Beziehung verbunden sind, und die Aufgaben (tasks), die notwendig sind, um die gesetzten Ziele zu erreichen (Sonesh et al., 2015). Uneinheitlich sind die Befunde zum Einfluss der relevanten Faktoren der Working Alliance auf den Coaching-Erfolg hingegen in der Studie von Gessnitzer und Kauffeld (2015). So zeigt sich hier bei den erhobenen Fragebogendaten kein Zusammenhang zwischen der Wahrnehmung der Working Alliance seitens der Coachees und der Zielerreichung. Bei der Einschätzung der Working Alliance durch den Coach bestätigt sich hingegen ein signifikant positiver Zusammenhang mit der Zielerreichung. Ein anderes Bild vermittelt hingegen die Auswertung der Beobachtungsdaten in dieser Studie. Während der Aspekt des bond, verstanden als Verbindung, die sich zwischen Coach und Coachee entwickelt, hier keinen Zusammenhang mit den Ergebnissen aufweist, zeigt sich bei durch Coachees initiierten Zielen und Aufgaben ein signifikant positiver Zusammenhang mit der Coaching-Wirkung. Hingegen hat es einen signifikant negativen Effekt auf den Coaching-Erfolg, wenn die Initiative der Ziel- bzw. Aufgabenklärung direkt vom Coach ausgeht. Als weitere Facette eines starken Arbeitsbündnisses erweist sich eine enge Arbeitsbeziehung, die auf Verständnis und Übereinstimmung gründet. Hier konnten zwei Studien (Boyce et al., 2010; Gan \& Chong, 2015) sowie eine Metaanalyse (Sonesh et al., 2015) einen signifikant positiven Effekt aufzeigen. Sonesh et al. (2015) verdeutlichen in ihrer Metaanalyse, dass der Aufbau eines guten Verhältnisses, das sich durch gegenseitiges Verständnis, Zustimmung und durch eine Verbindung der Parteien ausdrückt, sich positiv auf den Informationsaustausch auswirkt, was relevant ist, um die gesetzten Ziele zu erreichen. Teilt der Coach Informationen mit dem Coachee, wird dieser dies aus seiner eigenen Perspektive erwidern. Zudem vermittelt die Qualität der Arbeitsbeziehung zwischen dem Verhalten des Coachee und dem Verständnis des Coaches und nimmt darüber hinaus Einfluss auf die Motivation des Coachee und das Verständnis des Coaches. Darüber hinaus konnten die Intensität der Auseinandersetzung mit der Thematik (Offermanns, 2004) und der Herausforderungscharakter der Arbeitsbeziehung (Ladegard \& Gjerde, 2014) als weitere Facetten des Arbeitsbündnisses bestätigt werden. 


\subsection{Merkmale des Coaching-Prozesses}

Was die Merkmale des Coaching-Prozesses angeht, liegt empirische Evidenz bezüglich der beiden Wirkfaktoren Arbeitstechniken und methodisches Vorgehen sowie Zielklärung und Zielorientierung im Coaching-Prozess vor. Bei der Determinante Arbeitstechniken und methodisches Vorgehen im Prozessverlauf konnten anhand einiger weniger dazu vorliegender Studien insgesamt sechs Facetten identifiziert werden, die einen signifikanten Einfluss auf die Coaching-Wirkung haben. Runde et al. (2005) konnten den Einfluss der individuellen Analyse und Anpassung bestätigen, das heißt die Ausrichtung des inhaltlichen und methodischen Vorgehens im Coaching-Prozess an der individuellen Persönlichkeit der Klienten und Klientinnen. Als weitere Facette erweist sich in zwei Studien (Behrendt, 2006; MacKie, 2014) der Einfluss der Ressourcenorientierung und -aktivierung. Hierbei geht es um die Aktivierung von Stärken und Fähigkeiten sowie um die Erhöhung der Kompetenzerwartung bei den Klientinnen und Klienten. Überraschend ist zudem der Befund der Untersuchung von Behrendt (2006), die auf einen signifikant negativen Einfluss der Problemaktualisierung auf die Coaching-Wirkung verweist. Demnach beeinflusst die Aktivierung der relevanten problematischen Muster bei den Klienten und Klientinnen, um diese so der Veränderung zugänglich zu machen, den Coaching-Erfolg negativ. Darüber hinaus konnte in jeweils einer Studie der signifikant positive Einfluss der Facetten bedarfs- und fachgerechter Einsatz von Methoden und Interventionstechniken (Kappenberg, 2008), Methodenvielfalt und -matching (de Haan et al., 2013) sowie Maßnahmen zur Umsetzungs- und Transferunterstützung seitens des Coaches (Kappenberg, 2008) nachgewiesen werden.

Als zweiter Wirkfaktor auf der Ebene der Merkmale des Coaching-Prozesses zeigt sich die Zielklärung und -orientierung. In den einbezogenen Studien konnten fünf Facetten dieser Determinante systematisiert werden. Den signifikant positiven Effekt der Facette »Herstellung von Orientierung im Coaching-Prozess« konnte Offermanns (2004) bestätigen. Näher spezifiziert wird diese anhand der Fähigkeit des Coaches, Ziele und Erwartungen der Klientinnen und Klienten zu Prozessbeginn zu klären. Mehrere Studien belegen zudem übereinstimmend die Erfolgsrelevanz einer elaborierten Gestaltung der Zielklärung durch den Coach (Offermanns, 2004; Jansen et al., 2004; Runde et al., 2005; Brauer, 2006; Kappenberg, 2008; de Haan \& Mannhardt, 2013; MacKie, 2014). Die Studie von Scoular und Linley (2006) konnte hingegen keinen Einfluss dieser Facette nachweisen. Als weitere erfolgsrelevante Facetten des Wirkfaktors der Zielklärung konnte je eine Studie den positiven Einfluss der regelmäßigen Zielerreichungskontrolle im Prozessverlauf (Brauer, 2006) sowie die 
Unterstützung der Klientinnen und Klienten bei der Zielerreichung (Ladegard \& Gjerde, 2014) aufzeigen. Mit Blick auf den Einfluss der Vereinbarung von Zielen und Aufgaben verweist die Studie von Gessnitzer und Kauffeld (2015) auf eine bedeutsame Differenzierung, denn es hat sich ein signifikant negativer Effekt auf den Coaching-Erfolg gezeigt, wenn die Initiative der Ziel- bzw. Aufgabenklärung direkt vom Coach ausgeht. Hingegen konnte ein signifikant positiver Einfluss bestätigt werden, wenn die zu bearbeitenden Ziele von der gecoachten Person selbst angeregt werden.

\section{Fazit und Ausblick}

Die hier vorgestellten Ergebnisse der Analyse bisheriger quantitativer Forschungsbefunde im Hinblick auf prozessbezogene Determinanten der Wirkung von Einzelcoaching verweisen auf deutliche Tendenzen, aber auch auf bestehende Forschungsdefizite. So konnte aufgezeigt werden, dass sich insbesondere die Arbeitsbeziehung als zentraler Faktor der Coaching-Wirkung erweist, was mehrfach bestätigt wurde. Aber auch hierzu besteht noch Forschungsbedarf, da beispielsweise der Einfluss von Faktoren wie Ehrlichkeit, Akzeptanz und Angstfreiheit der Arbeitsbeziehung bislang nicht empirisch untersucht wurde. Zudem konnte aufgezeigt werden, dass insbesondere die Erfolgsrelevanz der Zielklärung und -orientierung im Coaching-Prozess recht gut untersucht ist. Überraschend ist, dass in Bezug auf das im Coaching eingesetzte methodische Vorgehen und die verwendeten Arbeitstechniken aus empirischer Perspektive noch viele Fragen unbeantwortet sind und nur wenige Studien dazu vorliegen. So sind die in Wirkmodellen (z. B. Greif, 2008) modellierten Einflussfaktoren, wie Affektreflexion und -kalibrierung, ergebnisorientierte Problemreflexion, Praxisbezug oder Transparenz bezüglich Interventionstechniken und Methoden, bislang kaum empirisch untersucht worden. Wiederum besteht eine vergleichsweise gute empirische Evidenz beim Einfluss der kommunikativen Fähigkeiten des Coaches auf den Coaching-Erfolg. Aber für weitere in der einschlägigen Literatur häufig genannte erfolgswirksame Kompetenzen des Coaches, wie psychologische oder betriebswirtschaftliche Kenntnisse, motivationale Fähigkeiten, Berufs- und Felderfahrung oder personale Faktoren wie Empathie, Authentizität, Vertrauenswürdigkeit, mangelt es bislang an empirischer Evidenz.

Die Analyse systematisiert somit nicht nur die empirisch bestätigten prozessbezogenen Determinanten der Wirkung von Einzelcoaching, sondern verdeutlicht auch, dass aus empirischer Perspektive noch viele Fragen zu relevanten Wirkfaktoren und ihren Facetten unbeantwortet sind. 


\section{Literatur}

Baron, L., Morin, L. (2009). The coach-coachee relationship in executive coaching: A field study. Human Resource Development Quarterly, 20 (1), 85-106.

Behrendt, P. (2006). Wirkung und Wirkfaktoren von psychodramatischem Coaching - eine experimentelle Evaluationsstudie. Zeitschrift für Psychodrama und Soziometrie, 5 (1), 59-87.

Bordin, E. S. (1979). The generalizability of the psychoanalytic concept of the working alliance. Psychotherapy: Theory, Research \& Practice, 16 (3), 252-260.

Boyce, L. A., Jackson, R. J., Neal, L. J. (2010). Building successful leadership coaching relationships. Examining impact of matching criteria in a leadership coaching program. Journal of Mangement Development, 29 (10), 914-931.

Bozer, G., Joo, B.-K., Santora, J. C. (2015). Executive coaching: Does coach-coachee matching based on similarity really matter? Consulting Psychology Journal: Practice and Research, 67 (3), 218-233.

Bozer, G., Sarros, J. C., Santora, J. C. (2014). Academic background and credibility in executive coaching effectiveness. Personnel Review, 43 (6), 881-897.

Brauer, Y. (2006). Zielvereinbarungen beim Coaching. Eine empirische Untersuchung aus Kundensicht. Saarbrücken: VDM.

Gan, G. C., Chong, C. W. (2015). Coaching relationship in executive coaching: A malaysian study. Journal of Management Development, 34 (4), 476-493.

Gatling, A. (2014). The authentic leadership qualities of business coaches and its impact on coaching performance. International Journal of Evidence Bases Coaching and Mentoring, 12 (1), 27-46.

Gessnitzer, S., Kauffeld, S. (2015). The working alliance in coaching: Why behavior is the key to success. The Journal of Applied Behavioral Science, 51 (2), 177-197.

Greif, S. (2008). Coaching und ergebnisorientierte Selbstreflexion: Theorie, Forschung und Praxis des Einzel- und Gruppencoachings. Göttingen: Hogrefe.

Haan, E. de, Culpin, V., Curd, J. (2011). Executive coaching in practice: What determines helpfulness for clients of coaching? Personnel Review, 40 (1), 24-44.

Haan, E. de, Duckworth, A., Birch, D., Jones, C. (2013). Executive coaching outcome research: The contribution of common factors such as relationship, personality match, and self-efficacy. Consulting Psychology Journal: Practice and Research, 65 (1), 40-57.

Haan, E. de, Mannhardt, S. (2013). Sie wollten schon immer wissen, was Führungskräfte-Coaching erfolgreich macht? Management Summary. www.sonja-mannhardt.de/wp-content/ uploads/2013/06/De-Haan_Management-Summary_HR1.pdf [10.6.2016].

Ianiro, P., Kauffeld, S. (2012). Wann stimmt die "Chemie« im Coaching? Untersuchungen zur gemeinsamen »Augenhöhe« von Coach und Klient. Coaching-Magazin, (1), 44-48.

Ianiro, P., Lehmann-Willenbrock, N., Kauffeld, S. (2015). Coaches and clients in action: A sequential analysis of interpersonal coach and client behavior. Journal of Business and Psychology, 30 (3), 435-456.

Jansen, A., Mäthner, E., Bachmann, T. (2004). Erfolgreiches Coaching. Wirkfaktoren im Einzel-Coaching. Kröning: Asanger.

Kappenberg, E. S. (2008). A model of executive coaching: Key factors in coaching success. Dissertation. Claremont Graduate University, Claremont, California.

Künzli, H. (2005). Wirksamkeitsforschung im Führungskräfte-Coaching. Organisationsberatung, Supervision, Coaching, 12 (3), 231-243.

Künzli, H. (2009). Wirksamkeitsforschung im Führungskräfte-Coaching. Organisationsberatung, Supervision, Coaching, 16 (1), 4-18.

Künzli, H., Stulz, N. (2009). Individuumsorientierte Coaching-Forschung. In B. Birgmeier (Hrsg.), Coachingwissen. Denn sie wissen nicht, was sie tun? (S. 159-170). Wiesbaden: VS Verlag für Sozialwissenschaften. 
Ladegard, G., Gjerde, S. (2014). Leadership coaching, leader role-efficacy, and trust in subordinates. A mixed methods study assessing leadership coaching as a leadership development tool. The Leadership Quarterly, 25 (4), 631-646.

MacKie, D. (2014). The effectiveness of strength-based executive coaching in enhancing full range leadership development: A controlled study. Consulting Psychology Journal: Practice and Research, 66 (2), 118-137.

Mühlberger, M. D., Traut-Mattausch, E. (2015). Leading to effectiveness: Comparing dyadic coaching and group coaching. The Journal of Applied Behavioral Science, 51 (2), 199-230.

Offermanns, M. (2004). Braucht Coaching einen Coach? Eine evaluative Pilotstudie. Stuttgart: ibidem.

Runde, B., Bastians, F., Weiss, U. (2005). Coaching und Supervisionsmaßnahmen des sozialwissenschaftlichen Dienstes der Polizei NRW. Polizei \& Wissenschaft, 6 (3), 40-51.

Schmidt, T., Keil, J.-G. (2004). Erfolgsfaktoren beim Einzel-Coaching. Ein Screening der Coachinglandschaft aus Sicht von Coachingnehmern. Organisationsberatung, Supervision, Coaching, 11 (3), 239-252.

Schreyögg, A. (2014). Empirische Erkundungen zum Coaching. Organisationsberatung, Supervision, Coaching, 21 (1), 1-2.

Scoular, A., Linley, A. (2006). Coaching, goal-setting and personality type: What matters? The Coaching Psychologist, 2 (1), 9-11.

Sonesh, S. C., Coultas, C. W., Marlow, S. L., Lacerenza, C. N., Reyes, D., Salas, E. (2015). Coaching in the wild: Identifying factors that lead to success. Consulting Psychology Journal: Practice and Research, 67 (3), 189-217.

Wechsler, T. (2012). Das coachingtool-spezifische Wirkfaktorenmodell. Organisationsberatung, Supervision, Coaching, 19 (4), 405-423 


\section{"ITurning Duty into Joy!॥}

Optimierung der Selbstregulation im Coaching durch Motto-Ziele

Julia Weber

Der Beitrag beschreibt theoretische Grundlagen und wissenschaftliche Untersuchungen zu den Motto-Zielen, einem neuen Zieltyp, der im Rahmen der theoretischen Überlegungen und praktischen Erfahrungen mit dem Selbstmanagement-Training nach dem Zürcher Ressourcen Modell ZRM entwickelt wurde. Durch die Verankerung im Unbewussten ermöglichen es Motto-Ziele, dass Menschen Handlungen in Selbstregulation ausführen.

Seit Jahren ist die Frage, wie Ziele formuliert sein müssen, um handlungswirksam zu werden, Gegenstand psychologischer Forschung (Fujita \& MacGregor, 2012). In vielen Therapieschulen, Beratungs- und Coaching-Methoden gehört das Formulieren von Zielen zum festen Handwerkszeug. Eine gängige Meinung zur Zielformulierung ist, dass Ziele so konkret wie möglich formuliert werden sollten (Locke \& Latham, 2013).

In diesem Beitrag widmen wir uns einem neuen Typus von Ziel, mit dem intrinsische Motivation sichergestellt, Selbstregulation erzeugt und Einstellungsänderungen angeregt werden können (Storch, 2009). Dieser Zieltyp wurde im Rahmen der theoretischen Überlegungen und praktischen Erfahrungen mit dem Selbstmanagement-Training nach dem Zürcher Ressourcen Modell ZRM entwickelt (Storch \& Krause, 2014). Unter Coaching werden im ZRM professionelle Beratungs-, Unterstützungs- und Begleitmaßnahmen verstanden, die Individuen befähigen, selbstbestimmte Wünsche und Ziele in Situationen des beruflichen und privaten Alltags nachhaltig umzusetzen (Meier \& Storch, 2013). ZRM-Coaching basiert auf der Idee, dass sich jeder Mensch grundsätzlich aus sich selbst heraus entwickeln kann, wenn er dort abgeholt wird, wo er im Moment ist, und zu den »richtigen « Schritten auf seiner Reise von Veränderungsabsichten und Wünschen zum Handeln begleitet wird (Krause \& Storch, 2006). ZRM-Coaching zeichnet sich durch fünf Kernelemente aus: Bedürfnisorientierung, Zielorientierung, Ressourcenorientierung, Handlungsorientierung und Nachhaltig- 
keit. Der Schwerpunkt dieses Beitrags liegt bei der Zielorientierung - beim Einsatz und bei der Formulierung von Zielen im Coaching. Dabei geht es um einen spezifischen Zieltypus: um die Motto-Ziele. Bei der Formulierung eines Motto-Ziels findet die Zerlegung in konkrete Verhaltensweisen nicht statt. Durch Motto-Ziele wird kein genauer, konkreter Plan vorgegeben, es geht vielmehr um die innere Haltung des Handelnden, aus der heraus situationsangepasstes Verhalten generiert wird. Im Folgenden werden zunächst die wissenschaftlich fundierten Theorien zu diesem neuen Zieltyp vorgestellt.

\section{Zwei Systeme: der Verstand und das Unbewusste}

Aus einer langen geistes- und psychologiegeschichtlichen Tradition heraus hat sich die Unterscheidung zwischen analytischem Denken und ganzheitlicher Intuition ergeben. Die Differenzierung zweier Systeme zur Erklärung von Phänomenen wie beispielsweise der Selbststeuerung und der Selbstwirksamkeit ist in mehreren psychologischen Modellen wiederzufinden (vgl. Überblick bei Sowden, Pringle \& Gabora, 2015; Carver \& Scheier, 2012); in der Psychologie werden sie unter dem Stichwort der »Zwei-Prozess-Theorien« untersucht. Dabei werden die beiden Systeme in der Literatur unterschiedlich bezeichnet: "hot « und »cool« (Metcalfe \& Mischel, 1999), »impulsiv« und »reflexiv« (Strack, Deutsch \& Krieglmeyer, 2009), »System 1« und »System 2 (Kahneman, 2012). Im Folgenden werden die Begriffe »Verstand « und »Unbewusstes« benutzt. Tabelle 1 gibt einen Überblick über einige wesentliche Unterschiede in der Arbeitsweise von Unbewusstem und bewusstem Verstand (Storch \& Krause, 2014).

Tabelle 1: Vergleich von Verstand und Unbewusstem

\begin{tabular}{|l|l|l|}
\hline & Verstand & Unbewusstes \\
\hline Verarbeitungsmodus & bewusst & unbewusst \\
\hline Geschwindigkeit & langsam & schnell \\
\hline Kommunikationsmittel & Sprache & somatische Marker (Gefühl) \\
\hline Informationsverarbeitung & seriell & parallel \\
\hline Bewertung & richtig/falsch & mag ich/mag ich nicht \\
\hline
\end{tabular}

Der Verstand arbeitet langsam und genau. Hat er eine Information verarbeitet, so ist der Mensch in der Lage, über Sprache seine Bewertung zu kommunizieren. Im besten Fall werden hierfür 900 Tausendstelsekunden benötigt, es kann aber manchmal auch Stunden, Tage oder Wochen gehen, bis dem Ver- 
stand etwas klar geworden ist. Durch serielle Informationsverarbeitung kann er immer nur eine Information auf einmal verarbeiten. Dieses System ist in der Lage, sehr genau und präzise zu arbeiten. Die Bewertung erfolgt aufgrund der Frage »was ist richtig, und was ist falsch«. Dabei spielen oftmals Erziehung und soziale Normen eine entscheidende Rolle.

Das Unbewusste arbeitet hingegen schnell. Innerhalb von 200 Tausendstelsekunden ist eine Bewertung oder ein Handlungsvorschlag vorhanden (Ferguson \& Porter, 2009). Allerdings wird, anders als beim Verstand, nicht über die Sprache kommuniziert, sondern über diffuse Gefühle, die von Damasio (2003) als somatische Marker bezeichnet werden. Die Bewertung erfolgt im Hinblick auf das Wohlbefinden des Gesamtorganismus, entweder als positives Gefühl (steuert das Appetenzverhalten) oder als negatives Gefühl (steuert das Aversionsverhalten). Die Informationsverarbeitung des Unbewussten verläuft parallel und ist so in der Lage, gleichzeitig sehr viele Informationen aus der In- und Umwelt zu verarbeiten und daraus eine Bewertung zu bilden. Die Bewertung erfolgt nach »mag ich und mag ich nicht«, nach »was ist zuträglich für das individuelle Wohlbefinden, und was ist störend«.

Verstand und Unbewusstes arbeiten also deutlich unterschiedlich, vor allem bewerten sie nach anderen Kriterien. In der Beratung, im Coaching oder in der Psychotherapie hat man es oft mit Klienten und Klientinnen zu tun, die darunter leiden, dass die Bewertung des Verstandes nicht mit der Bewertung des Unbewussten übereinstimmt. Grund ist die mangelnde Synchronisierung von Verstandesbewertung und der Bewertung des Unbewussten. Egal, welche Absicht ein Mensch verfolgt, die Tatsache, dass zwei Systeme an der Bewertung von Absichten und der Handlungssteuerung beteiligt sind, muss immer berücksichtigt werden.

Nun stellt sich die Frage, wie dies möglich ist. Wie kann die Synchronisation der beiden Systeme eingeleitet werden, und weshalb ist dies überhaupt wichtig mit Blick auf die Arbeit mit Zielen, auf die innere Haltung, die bei Motto-Zielen äußerst relevant ist? Dazu wird im Folgenden die Thematik der Zieltypen vertieft, werden verschiedene Zieltypen zugeordnet und zu den beiden Systemen in Bezug gebracht.

\section{Zieltypen}

Ziel ist nicht gleich Ziel. Je nachdem, wie Ziele sprachlich formuliert sind, lassen sie sich nach Typen unterscheiden (Deci \& Ryan, 2000). Storch (2008) hat als Visualisierungs- und Zuordnungshilfe verschiedener Zieltypen die Zielpyramide 
(vgl. Abbildung 1) entwickelt. Auf der Haltungsebene befinden sich die hohen, allgemeinen und globalen Zieltypen, die eher abstrakt formuliert sind. Auf der Verhaltensebene sind niedrige, spezifische und lokale Zielformulierungen anzusiedeln, die konkret formuliert sind. Die Ergebnisebene betrifft den Wunsch einer Person, der bewusst darüber Auskunft gibt, was sie als Ergebnis erreichen will. Storch betrachtet die Zielpyramide mit den drei Ebenen nicht als feste Struktur, sondern als Ordnungsversuch, »um eine Orientierung in die Vielfalt möglicher Sprachformen zu bringen, in die Menschen ihre Absichten packen können« (ebd., S. 71).

Die drei Ebenen der Zielpyramide beantworten unterschiedliche Fragen. Meist betreten Menschen die Zielpyramide in der Mitte, auf der Ergebnisebene. Hier wird die Frage beantwortet, was die Person tun will. Die Frage, warum sie dieses Ergebnis anstrebt, wird auf der Haltungsebene beantwortet. Hier werden die innere Haltung und die persönliche Einstellung geklärt, die hinter einer Absicht stehen. Auf der Verhaltensebene formulierte Ziele geben Auskunft über das Wie, das Wann und Wo einer beabsichtigten Handlung oder eines geplanten Verhaltens.

Haltung:

Warum?

Ergebnis:

Was?

Verhalten:

Wie? Wann? Wo?

Abbildung 1: Zielpyramide (vgl. Storch, 2008, S. 70) 
Die Verhaltensebene beinhaltet den konkreten, ergebnisbezogenen Zieltyp des Verstandes, der beispielsweise von Locke und Latham (2013) untersucht wurde. Als Ergebnis ihrer jahrzehntelangen Forschung kamen sie zu der Empfehlung, dass Ziele möglichst hoch, im Sinne von anforderungsreich, und möglichst spezifisch formuliert sein müssen, damit die Erfolgsaussicht möglichst hoch ist. In der Praxis hat sich diese Zielsetzungstheorie fest durch die Arbeit mit den sogenannten S.M.A.R.T.-Zielen etabliert. Auch im Therapie- und Coaching-Bereich wird häufig auf die Empfehlungen von Locke und Latham zurückgegriffen (Storch, 2009).

Neben den Zieltypen auf der Verhaltensebene gibt es solche auf der Haltungsebene, die in der wissenschaftlichen Zielpsychologie intensiv untersucht wurde. Diesem Zieltypus liegt eine Systematik zugrunde, die für die Entwicklung von Zielen nützlich ist und sowohl vom Selbst als auch vom Verstand unterstützt und getragen wird. Dieser Zieltyp hat jedoch in der Praxis noch keine weite Verbreitung gefunden. Es handelt sich um Zieltypen, die sich auf die innere Verfassung des Zielsetzenden beziehen. Beispielsweise untersucht Higgins (Schooler \& Higgins, 2012) in seiner »Regulatory Focus«-Theorie zwei Typen von Einstellungen, einen fördernden ("promotion «) und einen verhindernden ("prevention«) Fokus der Person. Kruglanski und Kopetz (2009) betonen, dass der Zieltyp auf der Haltungsebene im Unterschied zu spezifischen Zielen die Eigenschaft der Äquifinalität besitzt, dass also viele Handlungen zielführend sein können. Gollwitzer und Oettingen (2012) weisen darauf hin, wie wichtig es sei zu erkennen, dass Ziele mit demselben Inhalt durch unterschiedliche Haltungen in verschiedenen Weisen wirken.

Der Vergleich von Zielen auf der Haltungs- und auf der Verhaltensebene und die damit zusammenhängenden Auswirkungen auf die Zielerreichung und das Wohlbefinden der Person waren Gegenstand unterschiedlicher Studien (vgl. Gollwitzer \& Oettingen, 2012; Schutte, Searle, Meade \& Dark, 2012). Ferguson (2007) konnte in vier Studien nachweisen, dass die Haltung zu einem Ziel signifikant die Zielverfolgung vorhersagt, wobei sie auch betont (Ferguson \& Porter, 2009), dass Haltungen veränderbar sind.

Die Motto-Ziele aus dem Zürcher Ressourcen Modell (Storch \& Krause, 2014) werden in der Zielpyramide der Haltungsebene zugeordnet. Motto-Ziele sind eine spezifische Art von Haltungszielen. Sie ermöglichen es, Verstand und Selbst systematisch aufeinander abzustimmen und die unterschiedlichen Zieltypen in eine Form zu bringen, die die Einstellung zu einem Thema betrifft (Storch, 2009). Durch ein Motto-Ziel wird die Einstellung zu einem Thema geändert, und zwar so, dass sowohl das Unbewusste als auch der Verstand das Vorhaben unterstützen. 
Die Frage ist nun, welche sprachliche Form am besten geeignet ist, um das Unbewusste zu aktivieren und dadurch Selbstwirksamkeit und Selbstregulation zu erzeugen.

\section{Multiple Code Theory nach Bucci}

In der Multiple-Code-Theorie vereint Bucci (2002) psychoanalytisches Gedankengut mit den Überlegungen des Hirnforschers Damasio (2003). Die Theorie geht davon aus, dass Informationen aus der Umwelt vom menschlichen System grundsätzlich in zwei Arten von »Codes « wahrgenommen und verarbeitet werden: subsymbolisch (körperlich, emotional) und symbolisch. Informationen können über Körpergefühle, Bilder und/oder Worte codiert werden. Worte, die Informationen symbolisch verbal codieren, sind bewusstseinspflichtig. Körpergefühle, die subsymbolisch-emotional Informationen codieren, laufen unterhalb der Bewusstseinsschwelle ab. Bilder, die Informationen symbolisch-nonverbal codieren, können sowohl auf der bewussten als auch auf der unbewussten Ebene hervorgebracht werden (vgl. Abbildung 2).

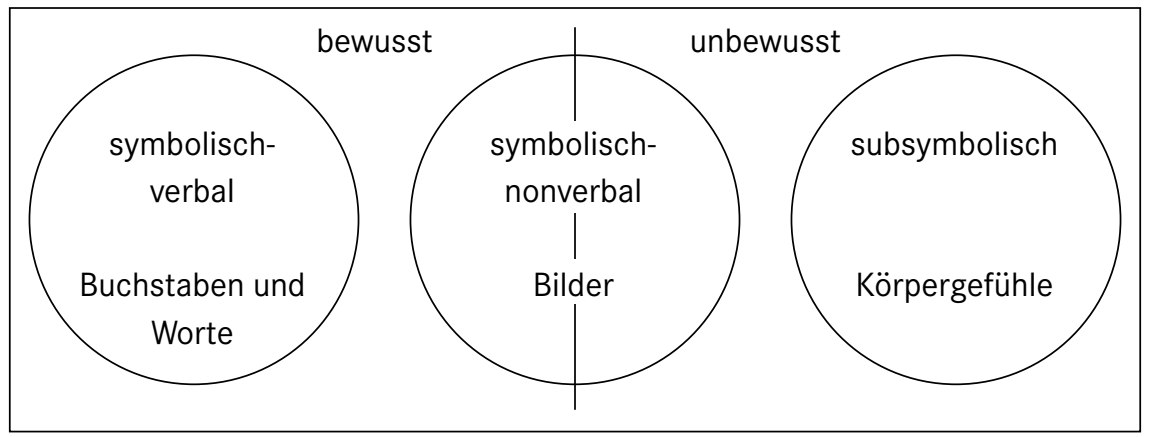

Abbildung 2: Informations-Codes nach Bucci (in Anlehnung an Storch, 2009)

Die drei Varianten der Verarbeitung sind miteinander über einen Vorgang verbunden, den Bucci (2002) den referenziellen Prozess nennt. Die Qualität der Verbindung dieser drei Systeme ist die Basis für gesundes psychisches Funktionieren (Bucci, 2005). Bilder sind der entscheidende Punkt in der Verbindung des subsymbolischen mit dem symbolisch-verbalen System, sie »sind der Dreh- und Angelpunkt im Informationsfluss zwischen vorsymbolischen Codes und symbolisch verbalen Codes« (Storch, 2009, S. 15). Die Übersetzung der Buchstaben in Körperempfindungen (Affekte) geschieht über Bilder. »An jedem Wort hängt ein Bild, und an jedem Bild hängt ein Gefühl« (ebd.). 


\section{Motto-Ziele in der Praxis}

Wenn diese Überlegungen auf das Bilden von Zielen angewendet werden, ergibt sich folgender Ablauf: Ein bewusst gefasstes Ziel muss mit dem Unbewussten abgestimmt werden, um Selbstregulation und intrinsische Motivation zu erzeugen. Um eine optimale Abstimmung mit dem Unbewussten zu sichern, müssen die bewusst gefassten Ziele in Worten ausgedrückt werden, die starke und eindeutige Bilder erzeugen, weil daran wiederum starke und eindeutige somato-affektive Signale gekoppelt sind, die man benötigt, um den Abstimmungsvorgang vornehmen zu können. Die sicherste Reihenfolge für den Bau von motivierenden Zielen lautet: zu dem bewussten Ziel ein Bild suchen, das starke positive Gefühle auslöst, dann zu dem Bild die passenden Worte erarbeiten, aus diesen Worten ein Haltungsziel bauen und dieses Sprachgebilde, das eng mit der Bilderwelt verbunden ist, mit den daran gekoppelten somato-affektiven Signalen aus der Körperwelt auf Maß schneidern. Das praktische Vorgehen mit dieser Methode ist bei Storch und Krause (2014) ausführlich erläutert. Ein Online-Tool unter www.ismz.ch erlaubt einen ersten Einblick in den Ablauf der Motto-Ziel-Bildung.

Motto-Ziele, die mit der Methode des Zürcher Ressourcen Modells individuell gebildet wurden, lauten zum Beispiel:

Ich genieße mein dickes Bärenfell und strecke alle viere von mir.

(Stressregulation)

In diesem Saustall hab ich das Sagen!

(Kritikgespräche mit Mitarbeitenden)

Ich bin der Kapitän, halte das Steuer fest in der Hand und segle zum Ziel.

(Masterarbeit schreiben)

Tigerlich tanze ich in die Männerwelt.

(Flirten und Partnersuche)

Ich bin der Gärtner - meine Pflanzen wachsen auch von allein.

(Delegieren von Arbeiten)

Aufgrund der oben besprochenen Äquifinalität von Zielen auf der Haltungsebene (Kruglanski \& Kopetz, 2009) erlauben Motto-Ziele, sofort nachdem sie gebildet wurden, spontan und situativ adäquates zielrealisierendes Handeln. Seine Wirkung entfaltet dieser Zieltypus durch die Veränderung der inneren Einstellung gegenüber einer Problemstellung (Storch \& Kuhl, 2012). In Fällen, in denen nicht nur ein neues Handlungsmuster gelernt, sondern auch noch 
ein altes Muster verlernt werden muss, müssen zusätzlich zum Motto-Ziel aber noch andere Maßnahmen getroffen werden. Dies betrifft auch Fälle, in denen die Umgebung des Klienten oder der Klientin situative Hinweisreize setzt, die alte, unerwünschte Verhaltensroutinen triggern (Wood \& Neal, 2007).

Motto-Ziele stellen intrinsische Motivation sicher, erzeugen Sinnerleben und Selbstbestimmung und regen Einstellungsänderungen an (Storch, 2009; Weber, 2013). Im Rahmen des Zürcher Ressourcen Modells werden sie sowohl im Coaching als auch im Training eingesetzt, so zum Beispiel beim Flirten (Weber \& Storch, 2012), in der psychosomatischen Rehabilitation (Storch \& Olbrich, 2011) oder auch im Umgang mit unangenehmen Pflichten und Prokrastination (Storch \& Weber, 2013). Zur Wirksamkeit der ZRM-Methode und der Motto-Ziele gibt es zahlreiche Studien (www.zrm.ch). Weber (2013) hat in ihrer Studie hochspezifische Ziele (Locke \& Latham, 2013) und Motto-Ziele bei unangenehmen Pflichten untersucht. Dabei stellte sich heraus, dass die konkreten Verhaltensziele nur den Verstand aktivieren, Motto-Ziele hingegen auch das Unbewusste ansprechen. Durch die Aktivierung des Unbewussten wird bei den Motto-Zielen die unangenehme Pflicht in Selbstregulation ausgeführt, die intrinsische Motivation steigt signifikant, und die erlebte Selbstbestimmung und der Optimismus der Probanden erhöhen sich deutlich. Auch verbessern Motto-Ziele die selbstgesteuerte Affektregulationskompetenz, was dazu führt, dass sich der negative Affekt reduziert und der positive Affekt erhöht. Dieses Ergebnis repliziert Ergebnisse anderer Studien, bei denen die selbstgesteuerte Affektregulationskompetenz untersucht wurde (vgl. Weber, 2013; Fröhlich, Kittel, Kruse, Greitemann \& Karoff, 2012). Weiter nimmt die wahrgenommene Belastung durch die unangenehme Pflicht durch Motto-Ziele signifikant ab. Ist das Unbewusste mit einem Vorhaben einverstanden, so erhöht dies die Zielbindung. Dies konnte im Vergleich mit konkreten Verhaltenszielen mehrfach nachgewiesen werden (vgl. Weber, 2013). In einer anderen Studie konnte mittels Speichelcortisolwert-Messung gezeigt werden, dass der subjektive Stress in einer Stresssituation (Trierer Stresstest) verringert wird (vgl. Storch, Gaab, Küttel, Stüssi \& Fend, 2007).

Für künftige Forschung wäre ein Desideratum die Durchführung von Langzeitstudien über einen Zeitraum von mehreren Jahren, die überprüfen, wie nachhaltig Motto-Ziele im Gegensatz zu konkreten spezifischen Zielen wirken. Für Coaching ist die Arbeit mit den Motto-Zielen vor allem bei folgenden Themenbereichen angebracht: Dem Klienten fehlt bei einem Thema die intrinsische Motivation, oder der Klient muss an von außen vorgegebenen Zielen arbeiten und diese erreichen. Die Stärke der ZRM-Methode liegt vor allem in der strukturierten Arbeit mit dem eigenen Unbewussten und deren theoretischen und wissenschaftlichen Fundierung. 


\section{Literatur}

Bucci, W. (2002). The referential process, consciousness, and the sense of self. Psychoanalytic Inquiry, 22 (5), 766-793.

Bucci, W. (2005). The interplay of subsymbolic and symbolic processes in psychoanalytic treatment. Commentary on paper by Steven H. Knoblauch. Psychoanalytic Dialogues, 15 (6), 855-873.

Carver, C. S., Scheier, M. (2012). Cybernetic control processes and the self-regulation of behavior. In R. M. Ryan, (Eds.), Oxford Handbook of Human Motivation (pp. 28-42). New York: Oxford University Press.

Damasio, A. R. (2003). Ich fühle, also bin ich. Die Entschlüsselung des Bewusstseins. München: List.

Deci, E. L., Ryan, R. M. (2000). The »What« and »Why« of goal pursuits: Human needs and the self-determination of behavior. Psychological Inquiry, 11 (4), 227-268.

Ferguson, M. J. (2007). On the automatic evaluation of end-states. Journal of Personality and Social Psychology, 92 (4), 596-611.

Ferguson, M. J., Porter, S. C. (2009). Goals and (implicit) attitudes. A social-cognitive perspective. In G. B. Moskowitz, H. Grant (Eds.), The psychology of goals (pp. 447-479). London: The Guilford Press.

Fröhlich, S. M., Kittel, J., Kruse, N., Greitemann, B., Karoff, M. (2012). Betsi - Beschäftigungsfähigkeit teilhabeorientiert sichern. Machbarkeitsstudie und Evaluation. Unveröffentlichter Abschlussbericht.

Fujita, K., MacGregor, K (2012). Basic goal distinctions. In H. Aarts, A. Elliot (Eds.), Goal-directed Behavior (pp. 85-114). New York: Psychology Press.

Gollwitzer, P. M., Oettingen, G. (2012). Goal pursuit. In R. M. Ryan (Ed.), The Oxford Handbook of Human Motivation (pp. 208-231). New York: Oxford University Press.

Kahneman, D. (2012). Schnelles Denken, langsames Denken. München: Siedler.

Krause, F., Storch, M. (2006). Ressourcenorientiert coachen mit dem Zürcher Ressourcen Modell $» \mathrm{ZRM}^{\circledR}{ }$. Psychologie in Österreich, 26 (1), 32-43.

Kruglanski, A. W., Kopetz, C. (2009). The Role of Goal Systems in Self-Regulation. In E. Morsella, J. A. Bargh, P. M. Gollwitzer (Eds.), Oxford Handbook of Human Action (pp. 350-367). New York: Oxford University Press.

Locke, E., Latham, G. (2013). New developments in goal setting and task performance. New York: Routledge.

Meier, R., Storch, M. (2013). Coaching mit dem Zürcher Ressourcen Modell ZRM ${ }^{\circledR}$. In E. Lippmann (Hrsg.), Coaching: angewandte Psychologie für die Beratungspraxis (3. Aufl., S. 74-86). Heidelberg: Springer.

Metcalfe, J., Mischel, W. (1999). A hot/cool-system analysis of delay of gratification: Dynamics of willpower. Psychological Review, 106 (1), 3-19.

Schooler, A. A., Higgins, E. T. (2012). Too much of a good thing? Trade-offs in promotion and prevention focus. In R. M. Ryan (Ed.), The Oxford Handbook of Human Motivation (pp. 65-84). New York: Oxford University Press.

Schutte, N. S., Searle, T., Meade, S., Dark, N. A. (2012). The effect of meaningfulness and integrative processing in expressive writing on positive and negative affect and life satisfaction. Cognition \& Emotion, 26 (1), 144-152.

Sowden, P. T., Pringle, A., Gabora, L. (2015). The shifting sands of creative thinking: Connections to dual-process theory. Thinking \& Reasoning, 21 (1), 40-60.

Storch, M. (2008). Rauchpause. Wie das Unbewusste dabei hilft, das Rauchen zu vergessen. Bern: Huber. Storch, M. (2009). Motto-Ziele, SMART-Ziele und Motivation. In B. Birgmeier (Hrsg.), Coachingwissen. Denn sie wissen nicht, was sie tun (S. 183-206). Wiesbaden: VS Verlag für Sozialwissenschaften. 
Storch, M., Gaab, J., Küttel, Y., Stüssi, A., Fend, H. (2007). Psychoneuroendocrine effects of resource-activating stress management training. Health Psychology, 26 (4), 456-463.

Storch, M., Krause, F. (2014). Selbstmanagement - ressourcenorientiert. Grundlagen und Trainingsmanual für die Arbeit mit dem Zürcher Ressourcen Modell (ZRM) (5., erw. u. vollst. überarb. Aufl.). Bern: Huber.

Storch, M., Kuhl, J. (2012). Die Kraft aus dem Selbst. Sieben PsychoGyms für das Unbewusste. Bern: Huber.

Storch, M., Olbrich, D. (2011). Das GUSI-Programm als Beispiel für Gesundheitspädagogik in Präventionsleistungen der Deutschen Rentenversicherung. In W. Knörzer, R. Rupp (Hrsg.), Gesundheit ist nicht alles - was ist sie dann? Gesundheitspädagogische Antworten (S. 111-126). Baltmannsweiler: Schneider Verlag Hohengehren.

Storch, J., Weber, J. (2013). Wolf packt La(h)ma. Wie Sie die Dinge zügig anpacken und konsequent erledigen. Bern: Huber.

Strack, F., Deutsch, R., Krieglmeyer, R. (2009). The two horses of behavior: Reflection and impulse. In E. Morsella, J. A. Bargh, P. M. Gollwitzer (Eds.), Oxford Handbook of Human Action (pp. 104117). New York: Oxford University Press.

Weber, J. (2013). Turning Duty into Joy! Optimierung der Selbstregulation durch Motto-Ziele. Dissertation. Lehrstuhl für Differentielle Psychologie und Persönlichkeitsforschung, Universität Osnabrück.

Weber, J., Storch, J. (2012). Tigerblick trifft Himbeerlächeln. Wie Ihnen das Unbewusste dabei hilft, lustvoll zu flirten. Bern: Huber.

Wood, W., Neal, D. T. (2007). A new look at habits and the habit-goal interface. Psychological Review, 114 (4), 843-863. 


\section{Mein Coaching!}

\section{Die Erfüllung des Bedürfnisses nach Autonomie}

Sandra J. Schiemann, Christina Mühlberger, Maximilian D. Mühlberger, Isabell Braumandl und Eva Jonas

Coaching gilt als Beratungsformat, bei dem an der Erreichung selbstkongruenter Ziele gearbeitet wird, an Zielen also, die im Einklang mit den eigenen Präferenzen, Einstellungen und Werten stehen. Durch ein Coaching sollte daher in besonderer Weise die autonome Selbstregulation der Coachees gefördert und damit ihr Bedürfnis nach Autonomie erfüllt werden. Entsprechend dieser Hypothese gingen die Autorinnen und der Autor dieses Beitrags bei drei Studien von der Annahme aus, dass im Coaching eine erhöhte autonome Selbstregulation erreicht und damit vor allem das Bedürfnis nach Autonomie angesprochen wird. In diesen drei Studien $\left(\mathrm{N}_{1}=73, \mathrm{~N}_{2}=221, \mathrm{~N}_{3}=89\right)$ wurde gezeigt, dass im Coaching tatsächlich besonders das Bedürfnis nach Autonomie - und weniger das nach Kompetenz oder sozialer Eingebundenheit - zentral ist. Theoretische Implikationen und praktische Empfehlungen, wie diese Ergebnisse auch in der Coaching-Praxis umgesetzt werden können, runden den Beitrag ab.

Im folgenden Beitrag wird anhand von drei Studien, die vom Autorenteam des Beitrags durchgeführt wurden, die Frage geprüft, inwiefern Coaching vor allem das psychologische Grundbedürfnis nach Autonomie im Gegensatz zu den beiden Bedürfnissen nach Kompetenz und sozialer Eingebundenheit anspricht (Deci \& Ryan, 2002; Jonas, Mühlberger, Böhm \& Esser, 2017). Dazu werden in einer ersten Studie die Bedürfnisse, die von den Beratungsformaten Coaching, Training und Supervision angesprochen werden, verglichen. Eine zweite Studie gilt der Frage, inwieweit auch in realen Coachings das Autonomiebedürfnis zentral ist. In einer dritten Studie betrachten wir die Autonomie als Prozess im Hinblick auf die Handlungsphasen von Heckhausen (1989). Die Annahme war, dass im Coaching eine erhöhte autonome Selbstregulation erreicht und damit vor allem das Bedürfnis nach Autonomie erfüllt wird. 


\section{Coaching als autonomiefördernde Personalentwicklungsmaßnahme}

Als Personalentwicklungsmaßnahme ist Coaching mittlerweile sehr gefragt und wird immer stärker beforscht (Kotte, Hinn, Oellerich \& Möller, 2016). Coaching wird in vielen Varianten für die verschiedensten Personen angeboten, zum Beispiel zur Führungskräfteentwicklung, als Karriere-Coaching, im Sport, bei Kindern und Jugendlichen, in der Wissenschaft und Politik (Wegener, Fritze \& Loebbert, 2013). Coaching wirkt sich positiv auf die Fähigkeiten der Coachees, deren individuelle Leistungen sowie den Unternehmenserfolg aus (Grover \& Furnham, 2016). Doch was genau ist Coaching?

Im Coaching unterstützt der Coach den Coachee bei der eigenen Lösungsfindung und der selbst gewollten Zielerreichung (Rauen, 2014). Die Ziele im Coaching sollten selbstkongruent sein - also im Einklang mit dem Selbst stehen (Greif, 2008). Sie sollten daher der eigenen Persönlichkeit und den eigenen Werten entsprechen (Grant, Passmore, Cavanagh \& Parker, 2010). Im Coaching werden diese selbstkongruenten Ziele berücksichtigt, und der Coach unterstützt seinen Coachee dabei, sein Handeln im Einklang mit diesen Zielen zu gestalten. Dies wird auch als das Erreichen von Autonomie bezeichnet - Selbstbestimmung des eigenen Verhaltens, das im Einklang mit dem Selbst ist (Ryan \& Deci, 2006). Coaching fördert also die Selbstbestimmung von Coachees (vgl. auch Bachkirova \& Smith, 2015), indem Coachees ihre individuellen Wünsche äußern, für sich stimmige Lösungen suchen und dadurch einen großen Gestaltungsspielraum im Beratungsprozess erhalten (Rauen, 2014).

Ein Coaching scheint daher vor allem die Autonomie zu bedienen. Autonomie ist nach Deci und Ryan (2002) ein psychologisches Grundbedürfnis und wird als ein Erleben von Selbstkongruenz und Selbstbestimmung definiert. Die beiden anderen bei Deci und Ryan (2002) genannten psychologischen Grundbedürfnisse nach Kompetenz (neue Fähigkeiten erlernen) oder sozialer Eingebundenheit (soziale Nähe/Verbundenheit erleben) stehen offenbar weniger im Vordergrund. Ein Erleben von eigenen Kompetenzen ist im Coaching wichtig, und Coachees können für ihr selbstkongruentes Ziel wertvolle Kompetenzen selbstbestimmt erweitern; es geht jedoch im Coaching nicht primär um den Kompetenzerwerb; der Erwerb oder eine Erweiterung von Kompetenzen würde im Training stattfinden, wo neue Fach- und Anwendungskenntnisse vermittelt oder bereits vorhandene Kompetenzen ausgebaut werden (Kauffeld, 2016). Ebenso ist ein Erleben von sozialer Eingebundenheit im Sinne einer guten Coach-Coachee-Beziehung während des Coachings wichtig, aber diese Beziehung dient der Autonomie des 
Coachee: Mithilfe einer vertrauensvollen und wertschätzenden Beziehung kann sich der Coachee öffnen und ganz er selbst sein (Baron \& Morin, 2009). So ist das vorrangige Ziel von Coaching nicht das Erlernen von Kompetenzen oder eine tiefe Bindung zwischen Coach und Coachee, sondern die Unterstützung des Coachee darin, selbstbestimmt und selbstkongruent zu handeln. Kompetenz und soziale Eingebundenheit scheinen hingegen in anderen Personalentwicklungsformaten relevanter zu sein (vgl. Jonas et al., 2017). Die zentrale Rolle der Autonomie im Coaching wird durch zwei Befunde verdeutlicht: So waren Coachees dadurch, dass sie in ihrer Autonomie unterstützt wurden, zufriedener (Losch, Traut-Mattausch, Mühlberger \& Jonas, 2016) und erreichten, wenn sie die Interaktion steuerten, eher ihre Ziele (Gessnitzer \& Kauffeld, 2015). Da Coaching als Beratungsformat auch einen Prozesscharakter hat (Rauen, 2014), stellt sich nun die Frage, wie das Bedürfnis nach Autonomie im Verlauf eines Coachings erfüllt wird.

\section{Die Erfüllung des Autonomiebedürfnisses als Prozess der autonomen Selbstregulation}

Coaching ist eine Prozessberatung, bei der Coachees schrittweise an der Erreichung ihrer eigenen und selbstkongruenten Ziele arbeiten (Greif, 2008). Sie lernen, ihren Fokus auf ihre Ziele zu lenken und die Bedingungen so zu gestalten, dass sie diese Ziele erreichen (Poetschki \& Wastian, 2014). Solche Prozesse der Zielverfolgung gliedern sich nach dem Handlungsphasenmodell von Heckhausen (1989) in mehrere Phasen, welche die Motivationsentstehung und -aufrechterhaltung beschreiben und die wir auch im Coaching wiederfinden (Greif, 2014) (vgl. Abbildung 1).

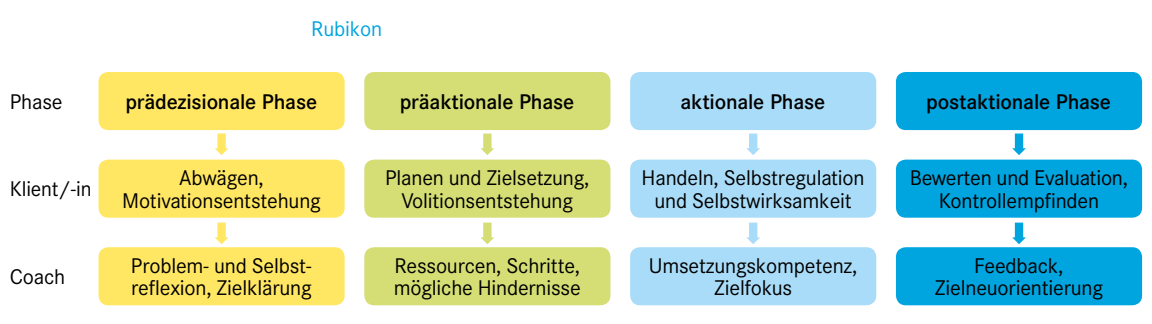

Abbildung 1: Das ans Coaching angepasste Handlungsphasen-Modell nach Heckhausen (1989)

In der ersten, der Abwäge-Phase (der prädezisionalen Phase) wird überlegt, wie bedeutsam und wertvoll das Ziel ist, aber auch wie wahrscheinlich es ist, dieses $\mathrm{zu}$ erreichen. Die bewusst gewordene persönliche Motivation wird durch ein 
dahinterliegendes Bedürfnis ausgelöst (Storch, 2004). Diese Motivation kann der Coach mit Interventionen im Sinne von ergebnisorientierter Problem- und Selbstreflexion, Zielklärung und Ressourcenaktivierung wecken. Bei einer positiven Bilanz folgt die Planungsphase (präaktionale Phase), in der die motivkongruenten, also zur persönlichen Motivation passenden Teilschritte für die Zielerreichung festgelegt und geplant werden. Durch den Wandel von Motivation in Volition, also eine Handlungsabsicht, wird der sogenannte »Rubikon-Fluss« überschritten, der als Symbol für Hindernisse steht. Um für den Coachee individuell wertvolle Ziele greifbar zu machen, kann der Coach gemeinsam mit dem Coachee die dafür benötigten Ressourcen und Schritte sowie mögliche Hindernisse - und wie sie sich umgehen lassen -, herausarbeiten. Ist alles vorbereitet, wird in der Umsetzungsphase (der aktionalen Phase) die Handlung initiiert und durchgeführt, bis das angestrebte persönliche Ziel erreicht wird. Zur selbstständigen Umsetzung sollte der Coachee das Gefühl haben, es schaffen oder gegebenenfalls mit einem »Plan B« neu versuchen zu können. Hierfür benötigt der Coach Methoden, welche die Umsetzungskompetenz des Coachee steigern - wie beispielsweise die langfristige Fokussierung der Energie auf das Ziel. Schließlich findet in der postaktionalen Phase Bewertung und Reflexion des Handlungsverlaufs und der Handlungsergebnisse statt, um Schlussfolgerungen auf zukünftiges Verhalten zu ziehen, beispielsweise auf die zukünftige persönliche Motivation. Um beim Coachee eine Selbstreflexion und realistische Selbstwirksamkeitsüberzeugung zu erzeugen, kann der Coach begleitend - zum Beispiel in Form eines Feedbackprozesses und durch Anstoßen von selbstständiger Abwandlung und Weiterführung der bisherigen Ziele - unterstützen (Greif, 2014; Heckhausen, 1989).

Wenn Bedürfnis, Motivation, Planung und Handlung miteinander im Einklang sind, ist es wahrscheinlich, ein motivkongruentes und autonomes Ziel zu erreichen (Pinsdorf, 2013).

\section{Unsere Forschungsergebnisse}

Die Annahme des Autorenteams war, dass ein Coaching besonders das Bedürfnis nach Autonomie anspricht und erfüllt. Das psychologische Grundbedürfnis nach Autonomie ist definiert als »die Neigungen zur Selbstregulation des Handelns und Stimmigkeit mit den eigenen Verhaltenszielen« (»the prospencities toward self-regulation of action and coherence in the organism's behavioral aims«; Deci \& Ryan, 2000, S. 253). Dass die drei psychologischen Grundbedürfnisse - nach Autonomie (entsprechend eigenen Präferenzen und Wertvorstellungen handeln), Kompetenz (Fähigkeiten und Können zeigen und steigern) 
und nach sozialer Eingebundenheit (Verbundenheit mit anderen Menschen fühlen) - erfüllt sind, ist essenziell für das eigene Wohlergehen. Die Grundbedürfnisse können jedoch abhängig vom sozialen Kontext unterschiedlich angesprochen und erfüllt werden (Deci \& Ryan, 2000). Unsere drei Studien, über die im Folgenden berichtet wird, unterstützen die Annahme, dass im sozialen Kontext Coaching vor allem das Bedürfnis nach Autonomie zentral ist. Die erste Studie diente dazu, herauszufinden, welches der drei Bedürfnisse im Coaching angesprochen wird. In einer zweiten Studie wurde betrachtet, inwiefern die drei Bedürfnisse in der realen Coaching-Interaktion erfüllt werden. Die dritte Studie betrachtete die Autonomieerfüllung, unterteilt in verschiedene Handlungsphasen der autonomen Selbstregulation von Heckhausen (1989).

\subsection{Erste Studie: Coaching, Training, Supervision - Welches Bedürfnis steht im Vordergrund?}

In der ersten Studie (Mühlberger \& Jonas, 2016) wurde erfasst, inwiefern Beratungsangebote zur Erfüllung der drei Grundbedürfnisse nach Autonomie, Kompetenz und sozialer Eingebundenheit beitragen und wodurch die Bedürfniserfüllung erklärt werden kann. 77 Personen beantworteten, wie wichtig ihnen die drei psychologischen Grundbedürfnisse nach Deci und Ryan (2002) waren (Autonomie: z. B. »Mir ist es wichtig, dass mir die Ziele, die ich verfolge, Freude bereiten «; Kompetenz: z. B. »Mir ist es wichtig, stets neue und interessante Fähigkeiten zu lernen «; soziale Eingebundenheit: z. B. »Mir ist es wichtig, mit meinen Interaktionspartnern gut klarzukommen«). Anschließend lasen die Teilnehmenden die Beschreibung zu den drei Beratungsangeboten Coaching, Training und Supervision. Basierend auf Greif (2008) und/oder Rauen (2014), dient das Coaching der Unterstützung zur Erreichung von Selbstreflexion und Selbstmanagement, das Training der (Weiter-)Entwicklung von Fach- und Methodenkompetenzen und die Supervision der systematischen Reflexion beruflichen Handelns. Die Teilnehmenden beantworteten daraufhin, inwieweit die Angebote ihre drei Bedürfnisse erfüllen würden. Dabei ging es um eigene Erwartungen an das jeweilige Format.

Die Ergebnisse zeigen, dass das Coaching-Angebot vor allem das Bedürfnis nach Autonomie ansprach. Erst danach spielten die Bedürfnisse nach Kompetenz und sozialer Eingebundenheit eine Rolle. ${ }^{1}$ Personen, die von vornherein

1 Beim Training wurde besonders das Bedürfnis nach Kompetenz angesprochen und bei der Supervision das Bedürfnis nach sozialer Eingebundenheit. Dies ist im Einklang mit den Annahmen von Jonas et al. (2016). 
einen starken Wunsch nach Autonomie verspürten, gaben zudem an, dass ein Coaching sowohl ihre Autonomie als auch ihre Kompetenz erhöhen würde. Mediationsanalysen zeigten, dass diese Bedürfniserfüllung durch eine erhöhte intrinsische Motivation und erhöhtes Vertrauen in den Coach erklärt werden konnte. Es stellt sich nun die Frage, wie es bei »realen « Coachings aussieht: Ist die Autonomie im Coaching das zentrale Bedürfnis, und wie wird dieses Bedürfnis erfüllt?

\subsection{Zweite Studie: Ist das Autonomiebedürfnis im Coaching zentral?}

Eine weitere Untersuchung sollte Aufschluss zur Rolle des Bedürfnisses nach Autonomie bei realen Coachings geben (Schiemann, Mühlberger, Braumandl \& Jonas, 2016). Im Rahmen der Ausbildung an der Universität Regensburg wurden die Coaching-Prozesse von 220 Coachees - 108 aus Peer-Coachings und 112 aus Klienten-Coachings - mit Fragebögen begleitet.

Die Skalen des Bochumer Inventars zur berufsbezogenen Persönlichkeitsbeschreibung (BIP; Hossiep \& Paschen, 2003), die ohnehin zur Stärken-Schwächen-Analyse verwendet wurden, nutzten wir, um die generellen Bedürfnisse der Coachees nach Autonomie, Kompetenz und sozialer Eingebundenheit zu erfassen. Am Ende des Coachings gaben die Coachees an, wie sie die Coach-Coachee-Interaktion in Bezug auf Autonomie (gemeinsame Prozessgestaltung, z. B. »Mein Coach regte mich dazu an, den Coaching-Prozess zu reflektieren und mitzugestalten«), Kompetenz (erlernte Fähigkeiten und Kompetenzen, z.B. »Durch das Coaching nutze ich meine Fähigkeiten und Fertigkeiten gezielter«) und soziale Eingebundenheit (positive Coach-Coachee-Beziehung, z. B. »Unsere Beziehung war von gegenseitiger Wertschätzung geprägt«) erlebt hatten und wie zufrieden sie mit dem gesamten Coaching waren.

Die Ergebnisse zeigten, dass sowohl in den Klienten- als auch in Peer-Coachings jede Art von Bedürfniserfüllung (Autonomie, Kompetenz, soziale Eingebundenheit) zu mehr Zufriedenheit mit dem Coaching führte. In den Peer-Coachings der Ausbildung waren die Coachees, die ein hohes Bedürfnis nach sozialer Eingebundenheit hatten, zufriedener, da dieses Bedürfnis in der Interaktion erfüllt wurde. In den Klienten-Coachings waren jedoch die Coachees, die ein hohes Bedürfnis nach Autonomie hatten, zufriedener, da dieses Bedürfnis in der Interaktion erfüllt wurde. ${ }^{2}$ Ergänzend zu Studie 1 konnte also auch im realen Klienten-Coaching gezeigt werden, dass das Bedürfnis nach

2 Die Bedürfnisse nach Kompetenz und sozialer Eingebundenheit wurden demgegenüber durch das Klienten-Coaching nicht angesprochen. 
Autonomie eine zentrale Rolle spielt. Da Coaching eine Prozessberatung mit verschiedenen Phasen ist, stellt sich nun folgende Frage: In welcher Phase wird das Bedürfnis nach Autonomie erfüllt, und was bleibt nach dem Abschluss des Coachings?

\subsection{Dritte Studie: Autonomieerfüllung im Prozess - Was bleibt mittelfristig an Autonomieförderung?}

In der dritten Studie (Mühlberger, Braumandl \& Jonas, 2015) wurde die wahrgenommene Autonomie in den vier Handlungsphasen nach Heckhausen (1989) untersucht. Befragt wurden 31 ehemalige Coachees (Studierende mit Teilnahme an einem Karriere-Coaching zwischen 2008 und 2011), 37 ehemalige Coaches (Studierende mit Karriere-Coaching-Ausbildung nach Braumandl und Dirscherl [2005] zwischen 2008 und 2011) und 21 Personen als Kontrollgruppe (Studierende von 2008 bis 2011 ohne Karriere-Coaching-Bezug). Wir nahmen an, dass Personen, die die Ausbildung als Coach durchlaufen hatten, am stärksten vom Coaching profitierten, da sie selbst nicht nur ein Peer-Coaching durchlaufen hatten, sondern sich danach durch die Anleitung des Klienten-Coachings zusätzlich intensiv mit der Unterstützung des Prozesses der Zielsetzung und Zielverfolgung beschäftigt hatten. Sie sollten also den Prozess der autonomen Selbstregulation am stärksten verinnerlicht haben. Die Teilnehmenden beantworteten zu jeder Handlungsphase Fragen in einer Online-Untersuchung (Abbildung 2, S. 112):

- Prädezisionale Phase: In der Phase des Abwägens und der Motivationsentstehung zeigte sich, dass vor allem die ehemaligen Coaches im Vergleich zu den Coachees und der Kontrollgruppe eine hohe Antriebskraft (VIE; Matsui, Kagawa, Nagamatsu \& Ohtsuka, 1977) und intrinsische Motivation (KIM; Wilde, Bätz, Kovaleva \& Urhahne, 2009) verspürten.

- Präaktionale Phase: Auch in der Zielsetzungsphase waren die ehemaligen Coaches am zielorientiertesten (Zielschwierigkeit und -spezifität, angelehnt an Pöhlmann \& Brunstein, 1997; Goal Commitment Scale; Hollenbeck, Williams \& Klein, 1989).

- Aktionale Phase: In der Umsetzungsphase schätzten sich die ehemaligen Coaches ebenfalls am umsetzungsstärksten ein (Test der Umsetzungskompetenz; Pelz, 2009).

- Postaktionale Phase: Auch bei den Fragen zur Reflexionsphase (FKK; Krampen, 1991; Fragen zum Umgang mit Feedback) schätzten sich die ehemaligen Coaches am höchsten ein. 


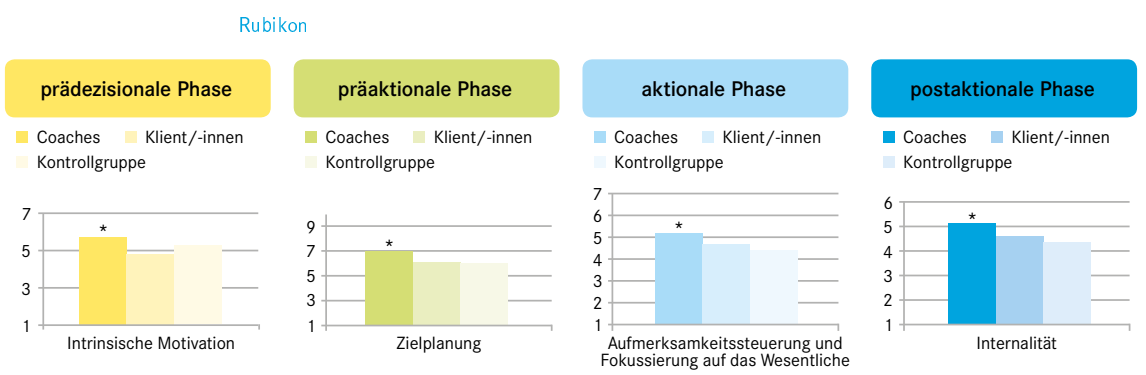

Abbildung 2: Ergebnisse zur autonomen Selbstregulation. ${ }^{*}=$ signifikant

Wie erwartet, haben vor allem die Coaches einen autonomen Selbstregulationsprozess verinnerlicht. Dies könnte daran liegen, dass sie in der Ausbildung sowohl als Coach wie auch als Coachee das Coaching mit Feedback und Supervision kennenlernten und sich so viel intensiver mit der Selbststeuerung auseinandergesetzt haben (vgl. Biberacher, Strack \& Braumandl, 2011). Um auszuschließen, dass diese Ergebnisse alternativ durch einen Selbstselektionseffekt zu erklären wären (das heißt, dass sich Personen mit hohen autonomen Selbstregulationsfähigkeiten bereits im Studium eher für eine Coaching-Ausbildung interessieren und daher auch beim Berufseinstieg eine hohe autonome Selbstregulationsfähigkeit aufweisen), führten wir eine Nachfolgeuntersuchung an 120 Studierenden des Masterstudiums Psychologie der Universität Salzburg durch. Die Ergebnisse zeigten, dass es vor der Coaching-Ausbildung keine signifikanten Unterschiede gab zwischen Studierenden, welche die Coaching-Ausbildung anstrebten, und solchen, die keine anstrebten (Mühlberger et al., 2015). Dies lässt vermuten, dass die signifikanten Ergebnisse tatsächlich durch die Ausbildung zum Karriere-Coach zustande kamen.

\section{Fazit und Ausblick}

Autonomie im Sinne einer selbst- und wertekongruenten Verhaltenssteuerung ist eines der drei psychologischen Grundbedürfnisse (Deci \& Ryan, 2002). Die Ergebnisse unserer Studien konnten zeigen, dass vor allem das Bedürfnis nach Autonomie im Coaching eine wichtige Rolle spielt. So wurde in Studie 1 (Umfrage) durch ein Coaching-Angebot das Bedürfnis nach Autonomie am stärksten angesprochen. In Studie 2 (reale Coachings) waren Klientinnen und Klienten mit einem hohen Bedürfnis nach Autonomie zufriedener mit dem Coaching, da ihr Bedürfnis nach Autonomie erfüllt wurde. In Studie 3 (Umfrage 
mit Coaches, Coachees und Personen ohne Coaching-Bezug) haben vor allem die Personen, die sowohl einmal Coachee als auch einmal Coach waren, die Autonomie anhand verschiedener Prozessschritte des Modells der Handlungsphasen von Heckhausen (1989) verinnerlicht.

In Bezug auf Motivation und Handeln sah bereits Storch (2004) die dahinterliegenden Bedürfnisse als unbewusste Kräfte. Diese Bedürfnisse lassen sich nach Deci und Ryan (2002) in drei psychologische Grundbedürfnisse einteilen, von denen vor allem das Bedürfnis nach Autonomie für das Beratungsformat Coaching zentral ist. Daher denken wir, dass es wichtig ist, in Coaching-Prozessen ein besonderes Augenmerk auf das Bedürfnis nach Autonomie und dessen Erfüllung zu legen. Dies kann unterstützt werden, indem der Coach als Prozessbegleiter und »Reflexionsanstifter « mit intellektueller Stimulierung und individueller Wertschätzung den Coachee in seiner autonomen Selbstreflexion begleitet (Traut-Mattausch, Mühlberger, Braumandl \& Jonas, 2016). Vor allem Techniken des motivationalen Interviewings, welche die Bedürfnisorientierung des Coachee bewusst ansprechen (Markland, Ryan, Tobin \& Rollnick, 2005), könnten hier hilfreich sein. Mit diesen Techniken kann der Coachee selbstbestimmt und selbstkongruent Ziele setzen und verfolgen.

Für künftige Forschung ist es wichtig, dass Coaching als ein interaktiver Prozess betrachtet wird (Rauen, 2014). Das Handlungsphasenmodell von Heckhausen (1989) beleuchtet jedoch nur die Handlungsphasen des Einzelnen und bezieht ihre Interaktion mit den Handlungsphasen eines anderen nicht ein. Für die Coaching-Forschung wäre daher das Loop2Loop-Modell von Jonas und Bierhoff (2016) interessant, da es die voneinander abhängigen Handlungsphasen zweier Personen veranschaulicht (vgl. Abbildung 3).

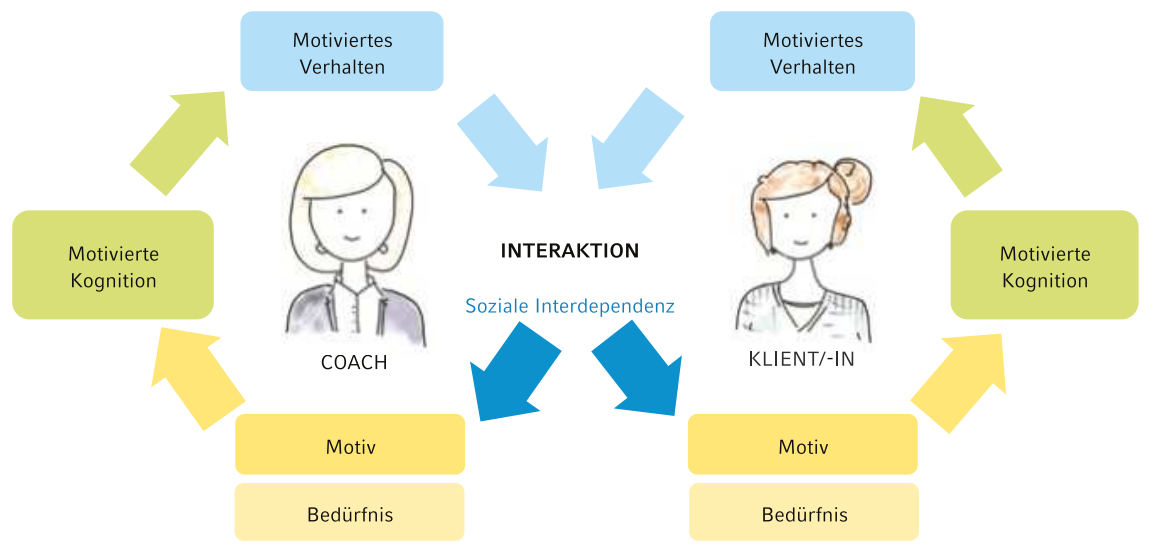

Abbildung 3: An das Coaching adaptiertes Loop2Loop-Modell 
Jede Person hat demnach einen Loop, der - wie im Handlungsphasenmodell von Bedürfnis über Motivation zu Verhalten führt. In diesem Loop resultiert aus einem Bedürfnis ein Motiv, das eine motivierte Kognition und daraufhin ein motiviertes Verhalten auslöst. Ein Beispiel soll dies verdeutlichen: Ein Coachee hat ein hohes Bedürfnis nach Autonomie und ist daher motiviert, über ein ihm wichtiges Ziel 1 zu sprechen; daher nimmt er sich vor, Ziel 1 gleich zu Beginn der Coaching-Sitzung anzusprechen (motivierte Kognition), und setzt dieses motivierte Verhalten zu Beginn der Coaching-Sitzung um.

In der Interaktion treffen die Loops beider Personen aufeinander, das motivierte Verhalten des einen wird für den anderen sichtbar, und die Loops können sich gegenseitig beeinflussen und voneinander abhängig werden (Loop-to-Loop/ Loop2Loop). Auf diese Weise entsteht eine soziale Interdependenz (Kelley \& Thibaut, 1978), bei der das Verhalten des anderen auf die eigene Zielerreichung Einfluss nimmt. Diese soziale Interdependenz kann also die eigene Bedürfniserfüllung beeinflussen und im weiteren Verlauf zu positiven oder negativen Interaktionen führen (Kelley et al., 2003). In unserem Beispiel könnte das motivierte Verhalten des Coachee, der Ziel 1 anspricht, auf einen Coach stoßen, der bereits einen Plan für das Coaching und somit eine andere motivierte Kognition hatte. Jetzt kann der Coach entweder auf den Coachee eingehen und damit seinen Loop ändern oder trotzdem an seinem Plan festhalten und seinen Loop beibehalten. Ebenso kann die Reaktion des Coaches (z. B. wie gut hört der Coach zu, wie sehr geht er auf das Thema »Ziel 1« ein, wie wertschätzend begegnet er dem Coachee) beeinflussen, ob sich der Coachee mit seinem Bedürfnis (nach Autonomie) gehört fühlt, und damit, wie viel und wie tief gehend der Coachee über Ziel 1 erzählt (motiviertes Verhalten).

Da das Bedürfnis nach Autonomie in der sozialen Interaktion Coaching wesentlich zu sein scheint, ist unseres Erachtens das autonomiefördernde Verhalten des Coaches zentral (z. B. Eingehen auf das Bedürfnis des Coachee), damit die Coaching-Interaktion erfolgreich verläuft.

\section{Literatur}

Baron, L., Morin, L. (2009). The coach-coachee relationship in executive coaching: A field study. Human Resource Development Quarterly, 20 (1), 85-106.

Biberacher, L., Strack, M., Braumandl, I. (2011). Coaching von Studierenden für Studierende: Evaluation einer Ausbildung zum Karriere-Coach. Wirtschaftspsychologie aktuell, 18 (3), 50-52.

Deci, E. D., Ryan, R. M. (2000). The »what« and »why« of goal pursuits: Human needs and the self-determination of behavior. Psychological Inquiry, 11 (4), 227-268. 
Deci, E. D., Ryan, R. M. (2002). Handbook of self-determination research. New York: University of Rochester Press.

Gessnitzer, S., Kauffeld, S. (2015). The working alliance in coaching: Why behavior is the key to success. Journal of Applied Behavioral Science, 51 (2), 177-197.

Grant, A. M., Passmore, J., Cavanagh, M., Parker, H. (2010). The state of play in coaching. International Review of Industrial \& Organizational Psychology, 25, 125-168.

Greif, S. (2008). Coaching und ergebnisorientierte Selbstreflexion. Göttingen: Hogrefe.

Greif, S. (2014). Coaching in Grenzfeldern zwischen Praxis und Wissenschaft: Vergleich von 50 Coaching-Ausbildern. Keynote am Coaching Kongress 2014 in Erding. www.coaching-koni gress.com/wp-content/uploads/Greif-Siegfried_Coaching-in-Grenzfeldern-zwischen-Praxis-und-Wissenschaft.pdf [11.3.2017].

Grover, S., Furnham, A. (2016). Coaching as a developmental intervention in organisations: A systematic review of its effectiveness and the mechanisms underlying it. PLOS ONE, 11 (7), 1-41.

Heckhausen, H. (1989). Motivation und Handeln (2. Aufl.). Berlin: Springer.

Hollenbeck, F. R., Williams, C. R., Klein, H. J. (1989b). An empirical examination of the antecedents of commitment to difficult goals. Journal of Applied Psychology, 74 (1), 18-23.

Hossiep, R., Paschen, N. (2003). BIP: Bochumer Inventar zur berufsbezogenen Persönlichkeitsbeschreibung. Göttingen: Hogrefe.

Jonas, E., Bierhoff, H.-W. (2016). Soziale Interdependenz und sozialer Austausch. In W. Bierhoff, D. Frey (Hrsg.), Kommunikation, Interaktion und Soziale Gruppenprozesse. Göttingen: Hogrefe.

Jonas, E., Mühlberger, C., Böhm, A., Esser, V. (2017). Soziale Austausch- und Interdependenzprozesse im Karrieremanagement: Training, Coaching, Mentoring und Supervision in einem sozialpsychologischen Vergleich. In S. Kauffeld, D. Spurk (Hrsg.), Handbuch Laufbahnmanagement und Karriereplanung. Heidelberg: Springer.

Kauffeld, S. (2016). Nachhaltige Personalentwicklung und Weiterbildung: Betriebliche Seminare und Trainings entwickeln, Erfolge messen, Transfer sichern (2., überarb. Aufl.). Berlin: Springer.

Kelley, H. H., Holmes, J. G., Kerr, N. L., Reis, H. T., Rusbult, C. E., Van Lange, P. A. M. (2003). An atlas of interpersonal situations. New York, NY: Cambridge University Press.

Kelley, H. H., Thibaut, J. W. (1978). Interpersonal relations: A theory of interdependence. New York, NY: Wiley.

Kotte, S., Hinn, D., Oellerich, K., Möller, H. (2016). Der Stand der Coachingforschung: Ergebnisse der vorliegenden Metaanalysen. Organisation, Supervision, Coaching, 23 (1), 5-23.

Krampen, G. (1991). FKK: Fragebogen zu Kompetenz und Kontrollüberzeugungen. Göttingen: Hogrefe.

Losch, S. Traut-Mattausch, E., Mühlberger, M. D., Jonas, E. (2016). Comparing the effectiveness of individual coaching, self-coaching, and group training: How leadership makes the difference. Frontiers in Psychology, 7 (629), 1-17.

Markland, D., Ryan, R. M.,Tobin, V. J., Rollnick, S. (2005). Motivational interviewing and selfdetermination theory. Journal of Social and Clinical Psychology, 24 (6), 811-831.

Matsui, T., Kagawa, M., Nagamatsu, J., Ohtsuka, Y. (1977). Validity of expectancy theory as a within-person behavioral choice model for sales activities. Journal of Applied Psychology, 62 (6), 764-767.

Mühlberger, C., Braumandl, I., Jonas, E. (2015). Vorteile beim Berufseinstieg durch eine Coaching-Ausbildung? Das Erreichen einer autonomen Selbstregulation im Coachingprozess. Unveröffentlichte Daten. Universität Salzburg.

Mühlberger, C., Jonas E. (2016). Bedürfniserfüllung in Coaching, Training und Supervision. Unveröffentlichte Daten. Universität Salzburg.

Pelz, W. (2009). Test der Umsetzungskompetenz (Volition). www.umsetzungskompetenzen.com [21.7.2014] 
Pinsdorf, K. (2013). How striving for your goals benefits others: directional motive inkongruence predicts organizational citizenship behavior as explained by self-determined motivation. Research Master's Psychology Thesis, University of Amsterdam.

Poetschki, J., Wastian, M. (2014). Coaching-Ziele klären und erreichen - was ist wirklich SMART? Erdinger Coaching-Kongress: »Zwischen Königsweg und Irrweg“, Poster, 20.-21.2.2014.

Pöhlmann, K., Brunstein, J. C. (1997). GOALS: Ein Fragebogen zur Messung von Lebenszielen. Diagnostica, 43 (1), 63-79.

Rauen, C. (2014). Coaching. Bd. 1. Göttingen: Hogrefe.

Ryan, R. M., Deci, E. L. (2006). Self-regulation and the problem of human autonomy: does psychology need choice, self-determination, and will? Journal of Personality, 74 (6), 1557-1585.

Schiemann, S., Mühlberger, C., Braumandl, I., Jonas, E. (2016). Bedürfniserfüllung im KarriereCoaching. Unveröffentlichte Daten. Universität Salzburg.

Storch, M. (2004). Resource-activating selfmanagement with the Zurich Resource Model (ZRM). European Psychotherapy, 5 (1), 27-64.

Traut-Mattausch, E., Mühlberger, M., Braumandl, I., Jonas, E. (2016). Wie führen Coaches? In R. Wegener, S. Deplazes, E.-M. Graf, S. Kotte, H. Künzli, A. Ryter, B. Uebelhart (Hrsg.), Coaching als individuelle Antwort auf gesellschaftliche Entwicklungen (S. 447-454). Wiesbaden: Springer.

Wegener, R., Fritze, A., Loebbert, M. (Hrsg.) (2013). Coaching-Praxisfelder: Forschung und Praxis im Dialog. Wiesbaden: Springer.

Wilde, M., Bätz, K., Kovaleva, A., Urhahne, D. (2009). Überprüfung einer Kurzskala intrinsischer Motivation (KIM). Zeitschrift für Didaktik der Naturwissenschaften, 15, 31-45. 


\section{Transferstärke-Coaching}

\section{Selbstlernkompetenz fördern und Lerntransfer sichern}

Axel Koch

Wieso schaffen es manche Menschen besser als andere, Lern- und Veränderungsimpulse aus Seminaren und Trainings umzusetzen? Das war die Ausgangsfrage, aus der sich das faktorenanalytisch ermittelte Transferstärke-Modell entwickelt hat. Es richtet den Fokus auf spezielle Einstellungen und Selbststeuerungsfertigkeiten, die umsetzungsstarke Menschen teilen. In diesem Beitrag wird das Transferstärke-Modell und das dazugehörige Coaching-Programm vorgestellt. Damit gelingt es, den Lerntransfer bei Soft-Skills-Trainings zu sichern und zugleich die Transferstärke der Teilnehmerinnen und Teilnehmer zu verbessern.

\section{Der selbstverantwortliche Top-Lerner ist gefragt}

Die Schulung sozialer Kompetenzen, wie Führung, Teambildung, Kommunikation, Konfliktmanagement, Verkauf oder Selbstführung, ist das Top-Thema in den Unternehmen. Dabei sind ein- bis zweitägige Seminare und Trainings mit einem Anteil von über sechzig Prozent das beliebteste Weiterbildungsformat. Das sind zentrale Ergebnisse der »managerSeminare«-Trendstudie »WeiterbildungsSzene Deutschland 2015«. Befragt wurden 1018 Trainerinnen und Trainer, Coaches und Weiterbildungsanbieter (Graf, 2015).

Gerade solche Soft-Skills-Schulungen haben jedoch den schlechten Ruf, dass die Teilnehmerinnen und Teilnehmer danach wenig bis gar nichts des Gelernten in ihrer Arbeit umsetzen. Gris (2008) spricht aus diesem Grund von einer »Weiterbildungslüge«. Seit rund zehn Jahren rangiert laut den SCIL-Trendstudien das Thema "Bildungsmaßnahmen transferförderlich gestalten« unter den Top drei der Herausforderungen für Personalentwickler. Bei diesen Studien werden alle zwei Jahre rund 150 Bildungsverantwortliche aus verschiedensten Unternehmen befragt (Euler \& Seufert, 2006; Diesner \& Seufert, 2010; Diesner \& Seufert, 2013; Fandel-Meyer \& Schneider, 2015). 
Als Lerntransfer gilt, dass in einer Fortbildungsmaßnahme gelerntes Wissen bzw. erworbene Fertigkeiten und Haltungen danach im Arbeitsalltag über eine längere Zeit gezeigt werden (Baldwin \& Ford, 1988, S. 64).

Das Problem des mangelnden Lerntransfers ist auch Gegenstand zahlreicher Forschungsarbeiten. Dabei zeigt sich, dass wesentliche Einflussfaktoren des Lerntransfers bereits identifiziert sind (z. B. Saks \& Belcourt, 2006; Grossman \& Salas, 2011; Saks, Salas \& Lewis, 2014; Weinbauer, 2015). Die Sichtung der Literatur fördert indessen einen interessanten Widerspruch zutage: Unternehmen beklagen auf der einen Seite die zu geringe Wirkung von Trainingsmaßnahmen, während sie auf der anderen Seite die Erkenntnisse aus der Lerntransferforschung zu wenig anwenden. Erklärbar wird dieser Sachverhalt durch die informellen Aussagen von Personalentwicklerinnen und Trainern. Danach fehlen in den Firmen Ressourcen für zeitaufwendige Schulungen. Vielmehr sollen Lernprozesse angesichts des operativen Drucks im Tagesgeschäft schnell und zeitsparend erfolgen. In der bereits erwähnten Studie "WeiterbildungsSzene Deutschland 2015 «zeigt sich dazu passend der Trend zur Verkürzung der Seminar- und Trainingsdauer. Rund 17 Prozent der Trainings sind bereits kürzer als einen Tag (Graf, 2015).

Die Anforderung besteht demzufolge zunehmend darin, die Beschäftigten in Unternehmen wirksam und zeitsparend in ihren Kompetenzen zu entwickeln. Viele Hoffnungen liegen auf unterschiedlichsten Formen von E-Learning. Zahlreiche neue Lern-Kurzformate wie zum Beispiel learning nuggets (Bergel, 2008; Gloger, 2009) oder microlearning (Gillies, 2013a) lassen sich gut mit den Möglichkeiten von E-Learning verbinden. Auch der Einsatz von Smartphones und Tablets - wir sprechen von mobile learning (Gillies, 2013b) - taugt dazu, sich im Arbeitsalltag flexibel das Wissen anzueignen, das gerade gebraucht wird. Besonders beliebt sind kurze Lernvideos (Gillies, 2014). Die Idee ist also: Jede und jeder kann seinen Lernprozess bedarfsgerecht steuern. Jeder und jede kann sich Zeit und Raum dafür einteilen.

Einige Vorreiterunternehmen wie die Deutsche Bahn (Pape, 2015; Eckelt \& Sauter, 2016) oder der Sportartikelhersteller Adidas (Reimann, 2015) denken radikal um. Formale Lernprozesse, Schulungen und lehrende Dozentinnen und Dozenten spielen in ihren Überlegungen nur noch eine geringe Rolle. Der "New Way of Learning" repräsentiert eine neue Kultur des selbstgesteuerten lebenslangen Lernens im Unternehmen, bei dem jede/r Beschäftigte die Verantwortung für die eigene Entwicklung übernehmen soll. Im Fokus steht selbstgesteuertes, arbeitsplatznahes und informelles Lernen, was auch Erpenbeck und Sauter (2013) als die Zukunft des Lernens sehen. 
Zusammengefasst, wünschen sich die Firmen also den selbstverantwortlichen und selbstgesteuerten Mitarbeitenden, der Lern- und Veränderungsimpulse nachhaltig wirksam umsetzt.

Genau an diesem Punkt setzt nun das Format des Transferstärke-Coachings an.

\section{Das Transferstärke-Modell}

Der Begriff »Transferstärke« ist definiert als persönliche Kompetenz, Lernund Veränderungsimpulse aus Fort- und Weiterbildungen selbstverantwortlich, erfolgreich und nachhaltig in der Praxis umzusetzen.

Das dahinterstehende Transferstärke-Modell wurde auf faktorenanalytischem Weg gewonnen. Am Anfang stand die Sichtung von Theorien, Modellen und empirischen Befunden aus der Therapie- und Lerntransferforschung, um einen Überblick zu erhalten, welche Einflussfaktoren aufseiten der Person den Lernund Veränderungserfolg bestimmen. Ergänzend zur Literaturrecherche wurden zwanzig Expertinnen und Experten (Personalentwickler, Führungskräfte, Trainerinnen) befragt.

Auf dieser Grundlage wurde ein erster Itempool zur Operationalisierung der Transferstärke gebildet. Schließlich lag nach einigen Pretests und Revisionen ein Itempool von 63 Items vor, die zehn theoretisch fundierten A-priori-Skalen zugeordnet waren. Als Antwortskala wurde eine sechsstufige Likert-Skala gewählt, bei der aus testtheoretischen Gründen nur deren Pole verbal verankert waren. Probanden konnten ihre Zustimmung zu den einzelnen Items auf der Skala von »trifft nicht zu« (0) bis »trifft voll zu« (5) angeben. Um Antworttendenzen zu vermeiden, war etwa die Hälfte der Items umgepolt, das heißt so formuliert, dass die Betonung nicht auf dem in der Literatur genannten lerntransfer- bzw. veränderungsförderlichen Aspekt lag.

Item-Beispiele sind:

- Handlungsempfehlungen, die nicht meinen Erfahrungen entsprechen, empfinde ich als graue Theorie.

- Um ein gewünschtes Verhalten zu erreichen, mache ich mir klar, in welchen Schritten ich am besten vorgehen muss.

- Es entmutigt mich, wenn ich in alte Verhaltensmuster zurückfalle.

Gemäß der klassischen Testtheorie (vgl. Lienert \& Raatz, 1989; Mummendey \& Grau, 2008) wurde diese Vortestversion an einer Stichprobe von 140 Probanden (69 Männer, 71 Frauen) im Alter von 17 bis 76 Jahren (Durchschnittsalter = 41,20 Jahre, $\mathrm{SD}=8,88)$ empirisch überprüft. Etwa die Hälfte der Probanden 
bekleidete eine Führungsposition. Eine explorative Faktorenanalyse (ScreeTest, Hauptkomponentenanalyse mit Varimax-Rotation) legte eine Drei- bzw. Vier-Faktoren-Lösung nahe, wobei die Drei-Faktoren-Lösung besser interpretierbar war (Koch, 2012).

Auf Basis dieser Ergebnisse erfolgte nach einer Revision der Vortestversion eine weitere empirische Überprüfung an einer Stichprobe von N $=1566$ Probanden (768 Männer, 798 Frauen) im Alter von 18 bis 67 Jahren (Durchschnittsalter $=39,09$ Jahre, $S D=10,35)$. Etwa ein Drittel hatte die Funktion einer Führungskraft. Eine weitere explorative Faktorenanalyse (Scree-Test, Hauptkomponentenanalyse mit Varimax-Rotation) brachte eindeutig Klarheit zugunsten einer Vier-Faktoren-Lösung mit abschließend 23 Items und einer aufgeklärten Varianz von 48,52 Prozent.

Diese Faktoren sind aufgrund von inhaltlichen Gesichtspunkten: »Offenheit für Fortbildungsimpulse« $(\mathrm{M}=3,72, \mathrm{SD}=, 89, \alpha=, 659)$, »Selbstverantwortung für den Umsetzungserfolg « $(\mathrm{M}=3,52, \mathrm{SD}=, 75, \alpha=, 789)$, »Rückfallmanagement im Arbeitsalltag « $(M=2,71, S D=1,02, \alpha=, 653)$ und »Positives Selbstgespräch bei Rückschlägen « $(M=3,47, S D=, 98, \alpha=, 745)$. Der Transferstärke-Gesamtwert $(\mathrm{M}=3,37, \mathrm{SD}=, 66, \alpha=, 850)$ ermittelte sich aus der Summe der vier Faktoren.

Im Folgenden werden diese vier Faktoren der Transferstärke näher beschrieben.

\section{Faktor 1 - Offenheit für Fortbildungsimpulse}

Offenheit ist die zentrale Grundlage für jede Weiterentwicklung. Menschen mit einem hohen Wert bei diesem Faktor haben eine positive Einstellung gegenüber Fortbildungen und damit verbundenen Lern- und Veränderungsimpulsen. Sie empfinden Inhalte und Übungen als nützlich. Sie lassen sich auf Neues und Ungewohntes ein. Es gelingt ihnen, gelernte Verhaltensregeln auf sich selbst passend anzuwenden.

\section{Faktor 2 - Selbstverantwortung für den Umsetzungserfolg}

Selbstverantwortung für den Umsetzungserfolg bedeutet, dass es aktive Bemühungen gibt, gelernte Inhalte in die Tat umzusetzen. Menschen mit einem hohen Wert bei diesem Faktor ergreifen die Initiative und sind aktiv, um aus ihrem bisherigen Trott zu kommen. Dies gelingt ihnen, weil sie gut in der Lage sind, sich selbst zu motivieren und neue Methoden und Fertigkeiten zu erarbeiten oder ungünstige Verhaltensweisen zu verändern. Sie machen sich klar, in welchen Schritten sie vorgehen müssen, um ein gewünschtes Verhalten zu erreichen. Sie haben die nötige Umsetzungsenergie und lassen sich auch nicht durch anfänglichen Mehraufwand und Anstrengungen von ihren Vorsätzen 
abbringen. Bereits in einer Fortbildung sind sie aktiv und nutzen Übungsmöglichkeiten, um an ihren Themen zu arbeiten. Aber auch nach einer Fortbildung bleiben sie aktiv und suchen sich gezielt Übungsmöglichkeiten und Informationen zur weiteren Vertiefung. Wenn sie nicht richtig vorankommen, holen Sie sich gezielt Hilfe für die Umsetzung der gelernten Inhalte. Dazu gehört auch, Menschen aus ihrem Umfeld einzubeziehen, die sie erinnern, wenn sie geplante Verhaltensänderungen nicht umsetzen - und die sie darin unterstützen, die Rahmenbedingungen förderlich zu gestalten.

\section{Faktor 3 - Rückfallmanagement im Arbeitsalltag}

Die Umsetzung von gelernten Inhalten steht meistens im zeitlichen Konflikt mit den Anforderungen des Tagesgeschäfts. Dieses fühlt sich dringend und wichtig an, sodass gute Vorsätze schnell ins Hintertreffen gelangen.

Menschen mit einem hohen Wert bei diesem Faktor beherrschen geeignete Strategien, um aus der »eigenen Komfortzone« gewohnter Handlungsweisen auszubrechen. Sie priorisieren die Umsetzung von Lernerkenntnissen und lassen sich nicht durch vermeintlich dringende Themen und das spontane Geschehen ablenken. Sie schätzen realistisch ein, was es an Zeit und Veränderungsaufwand braucht, und schaffen sich die erforderlichen Zeiträume. Ihnen gelingt es, sich auch unter Stress und Zeitdruck so zu steuern, dass sie sich an die Umsetzung neuer Denk- und Verhaltensweisen erinnern. Es gibt geeignete Vorbeugungsstrategien und Notfallpläne gegen Rückfälle.

\section{Faktor 4 - Positives Selbstgespräch bei Rückschlägen}

Das Bestreben, Gewohnheiten zu ändern oder Neues zu lernen, ist oft begleitet von Rückfällen in alte Muster, Fehlschlägen, unerwartet hohem Energieaufwand und Phasen der Frustration und Lustlosigkeit. Die Art des inneren Selbstgesprächs bei solchen Rückschlägen entscheidet darüber, ob Lern- und Veränderungsziele aufrechterhalten werden. Entscheidend ist eine positive und optimistische Grundeinstellung.

Menschen mit einem hohen Wert bei diesem Faktor sehen Rückfälle in alte Muster als normal an. Ihnen ist klar, dass Einstellungs- und Verhaltensänderungen nicht auf Anhieb gelingen - geschweige denn von heute auf morgen passieren. Sie sehen kleine und kleinste Fortschritte in ihren Bemühungen und »feiern« diese Erfolge. Sie sind zuversichtlich, dass sie früher oder später ihr Lern- und Veränderungsziel erreichen werden. All das trägt dazu bei, am Ball zu bleiben. Sie haben außerdem ein gutes Gefühl für den Nutzen, der sie erwartet, wenn sie ihr Ziel erreicht haben. 
Erste Validierungsstudien konnten bereits die Konstruktvalidität des Transferstärke-Messinstruments - Transferstärke-Analyse genannt - bestätigen. So wurde die konvergente Validität (Bühner, 2011, S. 64) mit der allgemeinen Selbstwirksamkeits-Skala überprüft (Schwarzer \& Jerusalem, 1999, S. 13). Gerade das Konstrukt der Selbstwirksamkeit gilt in Befunden der Lerntransferforschung übereinstimmend als wichtiger Prädiktor für den Transfererfolg (z. B. Weinbauer, 2015, S. 50; Chiaburu \& Lindsay, 2008).

Bei einer Stichprobe von 82 Probanden (44 Männer, 38 Frauen) im Alter von 21 bis 73 Jahren (Durchschnittsalter $=37,85$ Jahre, $S D=10,08$ ) zeigte sich eine hoch signifikante Korrelation ( $\mathrm{r}=, 588, \mathrm{p}=, 000,2$-seitig) zwischen den Gesamtwerten für Transferstärke und allgemeine Selbstwirksamkeit. Etwa ein Drittel der Probanden bekleidete eine Führungsposition.

Weitere Studien stützen die Validität des Gesamtkonstruktes bzw. einzelner Skalen (vgl. Kreutz, 2012; Schürf, 2012; Papenhoff, 2013; Pankow, 2013). Diese Studien werden hier nicht weiter ausgeführt, da sie sich auf die erste Version der Transferstärke-Analyse beziehen, der noch die Drei-Faktoren-Lösung zugrunde lag.

\section{Vorgehen beim Transferstärke-Coaching}

Betrachtet man die Prozentrangplätze der Teilnehmerinnen und Teilnehmer aus der bereits erwähnten Stichprobe von $\mathrm{N}=1566$ Probanden, dann haben nur zwanzig Prozent der Teilnehmenden einen überdurchschnittlichen Transferstärke-Wert. Aus der Modellsicht bedeutet dies, dass dieser Teilnehmerkreis in einem ausgeprägten Maße die erforderlichen Einstellungen und Fertigkeiten besitzen, die für den Lerntransfer bei Soft-Skills-Trainings bedeutsam für den Umsetzungserfolg sind.

Geht man nun aber davon aus, dass in der Praxis nur rund zwanzig Prozent der Trainingsteilnehmerinnen und -teilnehmer transferstark sind, gilt es, alle anderen bedarfsgerecht zu ihrem Transferstärke-Profil zu stärken und zu unterstützen, damit der Lerntransfer gesichert werden kann. Genau hier setzt das neue Format des Transferstärke-Coachings ein.

Das folgende Schaubild zeigt die Grundidee. Bisherige praktische Erfahrungen zeigen, dass es je nach Trainingsprogramm und geplantem Umfang verschiedene Möglichkeiten gibt, um den beschriebenen Ablauf bedarfsgerecht zu integrieren. 


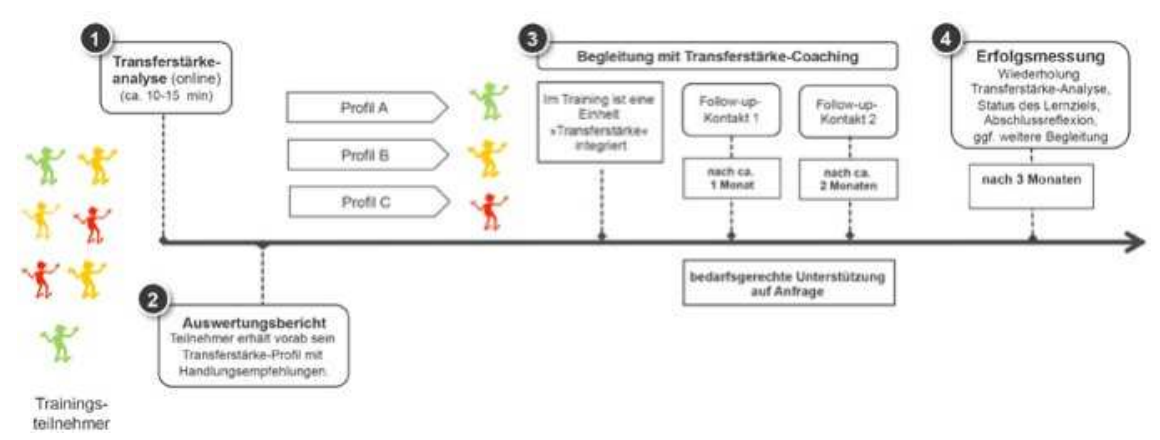

Abbildung 1: Vorgehen beim Transferstärke-Coaching

Der erste Schritt besteht darin, dass die Teilnehmerinnen und Teilnehmer eines Trainings sich einer Transferstärke-Analyse unterziehen. Sie umfasst die erwähnten 23 Items und verlangt eine Bearbeitungszeit von etwa fünf bis zehn Minuten.

Im zweiten Schritt bekommt der Teilnehmer oder die Teilnehmerin den persönlichen Auswertungsbericht zugesendet, damit er oder sie bereits vor dem Training Klarheit über das persönliche Transferstärke-Profil erhält. Die Trainerin hat in Form eines Gruppenprofils alle Teilnehmerinnen und Teilnehmer im Blick und weiß, wie sie angesichts der Transferstärke-Profile ein anstehendes Training passend designen muss. Dazu ein Beispiel: Wenn sich im Gruppenprofil zeigt, dass bei zahlreichen Teilnehmern und Teilnehmerinnen der Faktor »Aktives Rückfallmanagement « schwach ausgeprägt ist, kann sie zum Beispiel unterstützend spezielle Transferaufgaben für die Zeit nach dem Training einbauen, die bei den Teilnehmern und Teilnehmerinnen die Umsetzung des Gelernten fördern. Aufgrund der Profile bekommt die Trainerin auch die Möglichkeit, bedarfsgerecht vorab auf Teilnehmerinnen und Teilnehmer zuzugehen, wenn sich im Profil zum Beispiel fehlende Offenheit für das Training zeigt.

Die Teilnehmerinnen und Teilnehmer selbst werden durch den Auswertungsbericht vor dem Training sensibilisiert, welche Risiken bei ihnen für den Lerntransfer bestehen. Gleichzeitig erhalten sie bereits konkrete, evidenzbasierte Handlungsempfehlungen, wie sie die identifizierten Risikobereiche besser managen können. Diese Handlungsempfehlungen betreffen zum Beispiel Fertigkeiten des »aktiven Rückfallmanagements« (vgl. z. B. Marx, 1982; Marlatt \& Gordon, 1985; Marx, 1993) oder der Selbststeuerung (vgl. z. B. Gollwitzer \& Sheeran, 2006; Pachner, 2009; Oettingen \& Gollwitzer, 2010; Furtner \& Sachse, 2011).

Der dritte Schritt besteht darin, dass die Teilnehmerinnen und Teilnehmer wie sonst auch üblich - ins Training gehen. Darin ist eine Lerneinheit »Arbeit 
mit dem Transferstärke-Profil« integriert, die etwa drei Stunden umfasst. Die Trainerin führt als Beispiel für alle ein Transferstärke-Auswertungsgespräch mit einem Teilnehmer durch. Daran wird für alle Beteiligten gut erlebbar, wie sie mit ihrem eigenen Profil weiterarbeiten müssen, um ihren Lerntransfer sicherzustellen und zugleich ihre Transferstärke zu stärken. Im Anschluss erarbeitet jede und jeder für ein spezielles, selbst gewähltes Lernziel, wie zum Beispiel "Verhandlungspositionen durch Fragen besser klären « (im Rahmen eines Verhandlungstrainings), ihre oder seine transferwirksame Umsetzungsstrategie. Die Trainerin als Transferstärke-Coach unterstützt dabei.

Im Anschluss an das Training folgt die selbstständige Anwendungsphase von rund drei Monaten. Die Teilnehmerinnen und Teilnehmer reflektieren mithilfe einer Lernverlaufskurve ihren Umsetzungserfolg und holen sich bei Problemen bedarfsgerecht Unterstützung bei der Trainerin. In diesen Anwendungszeitraum sind zwei Follow-up-Kontakte fest integriert.

Solche Kontakte finden im Abstand von je einem Monat statt. Die Trainerin als Transferstärke-Coach fragt dabei typischerweise in einem etwa dreißigminütigen Telefonat nach, inwiefern die Umsetzung funktioniert hat und welche offenen Fragen es gibt. Durch dieses Transferstärke-Coaching lernt der Teilnehmer am Beispiel seines aktuellen und persönlich wichtigen Lernziels, wie er die Informationen aus seinem Auswertungsbericht nutzt, um den Umsetzungserfolg sicherzustellen. Im Mittelpunkt des Gesprächs steht, dass der Teilnehmer noch vertrauter und sicherer in der Anwendung der Handlungsempfehlungen wird und sich in den Risikobereichen seiner Transferstärke noch besser zu steuern lernt. Dabei ist das Coaching grundsätzlich als Hilfe zur Selbsthilfe angelegt und nutzt die empirisch bestätigten Wirkfaktoren des Coachings als Basiskonzept (vgl. Wechsler, 2012; Behrendt, 2012; Greif, Schmidt \& Thamm, 2012).

Alternativ zu einem Telefonat kann dieser Reflexionsteil bei Intervalltrainingsformaten auch in die nachfolgenden Trainingsmodule integriert werden.

Der vierte Schritt besteht schließlich in einer Erfolgsmessung. Dabei wiederholt der Teilnehmer nach etwa drei Monaten die Transferstärke-Analyse, um auf diesem Weg eine Rückmeldung zu bekommen, wie sich die eigene Transferstärke entwickelt hat. Außerdem wird der erreichte Stand für das aktuelle Lernziel reflektiert.

Der beschriebene Prozess des Transferstärke-Coachings ist das Ergebnis verschiedener explorativer Untersuchungen. Es hat sich gezeigt, dass es oft nicht reicht, Teilnehmern und Teilnehmerinnen nur ihren Transferstärke-Auswertungsbericht zur selbstständigen Bearbeitung zur Verfügung zu stellen. Dies bestätigt auch Gerathewohl (2015, S. 76). Diese Befunde decken sich mit der Annahme, dass gerade transferschwache Menschen erst die Kompetenzen auf- 
bauen müssen, die ihnen den Lern- und Umsetzungserfolg ermöglichen. Darüber hinaus tun sich viele Menschen mit Selbstcoaching schwer (Martens 2015, S. 59).

Einen ersten Beleg für die Wirksamkeit des Transferstärke-Coachings liefert eine Evaluationsstudie mit einer Experimentalgruppe (EG) und einer Kontrollgruppe (KG). Die Teilnehmerinnen und Teilnehmer wurden gebeten, für sich ein persönlich wichtiges Entwicklungsthema zu definieren.

Die Werte wurden zu drei Zeitpunkten gemessen: Es gab einen Vortest im September/Oktober 2014, einen ersten Nachtest im Januar/Februar 2015 und einen Nachtest 2 im September/Oktober 2015. An der Studie nahmen zwanzig Master-Studenten und -Studentinnen der Wirtschaftspsychologie teil. Die drei Männer und 17 Frauen im Alter zwischen 23 und 34 Jahren $(M=26,75$ Jahre, $\mathrm{SD}=3,16)$ waren überwiegend in der Funktion von Mitarbeitern und Mitarbeiterinnen in Unternehmen tätig (75\%) oder hatten aktuell kein festes Arbeitsverhältnis (20\%). Nur ein Teilnehmer hatte eine fachliche Führung inne. Dabei hatten sich 14 Probanden freiwillig für die Teilnahme in der Experimentalgruppe entschieden, sechs für die Kontrollgruppe. Drei Teilnehmende (einer EG, zwei KG) fielen beim Nachtest 2 aus der Studie heraus und konnten daher nicht berücksichtigt werden.

Die folgende Tabelle zeigt die Mittelwerte (MW) und Standardabweichungen (SD) für die einzelnen abhängigen Variablen pro Versuchsgruppe sowie die Ergebnisse der Tests der Innersubjekteffekte (Sphärizität angenommen) einer zweifaktoriellen Varianzanalyse mit Messwiederholung.

Tabelle 1: Zweifaktorielle Varianzanalyse mit Messwiederholung zur Überprüfung der Wirksamkeit des Transferstärke-Coachings. ${ }^{*}=$ signifikant mit $\mathrm{p}<, 05$; ${ }^{* *}=$ hoch signifikant mit $\mathrm{p}<, 01$

\begin{tabular}{|l|l|l|l|l|l|}
\hline Variablen & $\begin{array}{l}\text { MW (SD) } \\
\text { Vortest: t1 }\end{array}$ & $\begin{array}{l}\text { MW (SD) } \\
\text { Nachtest 1: } \\
\text { t2 }\end{array}$ & $\begin{array}{l}\text { MW (SD) } \\
\text { Nachtest 2: } \\
\text { t3 }\end{array}$ & $\begin{array}{l}\text { Zeitpunkt } \\
\mathbf{p}\end{array}$ & $\begin{array}{l}\text { Zeitpunkt* } \\
\text { Gruppe } \\
\text { p }\end{array}$ \\
\hline $\begin{array}{l}\text { Transferstärke } \\
\text { EG } \\
\text { KG }\end{array}$ & $\begin{array}{l}2,59(, 69) \\
3,25(, 66)\end{array}$ & $\begin{array}{l}3,49(, 50) \\
3,12(, 72)\end{array}$ & $\begin{array}{l}3,52(, 47) \\
3,03(, 55)\end{array}$ &, $015^{\star}$ &, $000^{* *}$ \\
\hline $\begin{array}{l}\text { Offenheit } \\
\text { EG }\end{array}$ & $3,25(, 94)$ & $3,84(, 52)$ & $3,98(, 44)$ &, 102 &, 102 \\
KG & $3,79(, 77)$ & $3,79(1,07)$ & $3,79(, 51)$ & & \\
\hline $\begin{array}{l}\text { Selbstverant- } \\
\text { wortung } \\
\text { EG } \\
\text { KG }\end{array}$ & $2,76(, 83)$ & $3,61(, 61)$ & $3,63(, 54)$ &, 102 &, $001^{* *}$ \\
\hline
\end{tabular}




\begin{tabular}{|c|c|c|c|c|c|}
\hline Variablen & $\begin{array}{l}\text { MW (SD) } \\
\text { Vortest: } \mathrm{t1}\end{array}$ & $\begin{array}{l}\text { MW (SD) } \\
\text { Nachtest 1: } \\
\text { t2 }\end{array}$ & $\begin{array}{l}\text { MW (SD) } \\
\text { Nachtest 2: } \\
\text { t3 }\end{array}$ & $\begin{array}{l}\text { Zeitpunkt } \\
\mathrm{p}\end{array}$ & $\begin{array}{l}\text { Zeitpunkt* } \\
\text { Gruppe } \\
p\end{array}$ \\
\hline $\begin{array}{l}\text { Rückfall- } \\
\text { management } \\
\text { EG } \\
\text { KG }\end{array}$ & $\begin{array}{l}1,93(1,00) \\
2,54(1,09)\end{array}$ & $\begin{array}{l}2,96(, 92) \\
2,20(1,03)\end{array}$ & $\begin{array}{l}3,05(, 99) \\
2,17(, 54)\end{array}$ &, 114 &, $001^{*}$ \\
\hline $\begin{array}{l}\text { Positives } \\
\text { Selbstgespräch } \\
\text { EG } \\
\text { KG }\end{array}$ & $\begin{array}{l}2,44(, 93) \\
3,17(1,08)\end{array}$ & $\begin{array}{l}3,56(, 64) \\
3,13(1,18)\end{array}$ & $\begin{array}{l}3,44(, 66) \\
3,31(, 74)\end{array}$ &, $042^{*}$ &, $014^{\star}$ \\
\hline
\end{tabular}

Wie aufgrund der Anwendung des Transferstärke-Coachings zu erwarten war, zeigen die Experimental- und die Kontrollgruppe unterschiedliche Verläufe über die drei Messzeitpunkte (vgl. Tabelle 1). Die Werte der Experimentalgruppe steigen an und übertreffen diejenigen der Kontrollgruppen zum dritten Messzeitpunkt. Entsprechend wurden die Interaktionen zwischen Zeitpunkt und Gruppe weitestgehend signifikant - bis auf eine Ausnahme bei der abhängigen Variablen »Offenheit«. Die Transferstärke hat sich also in der Experimentalgruppe deutlicher verbessert als in der Kontrollgruppe. Es besteht darüber hinaus kein signifikanter Haupteffekt zwischen den beiden Gruppen.

Dabei ist zu erwähnen, dass es beim Vortest keine signifikanten Unterschiede bei den abhängigen Variablen zwischen der Experimental- und der Kontrollgruppe gab ( $\mathrm{p}=$,057, 2-seitig).

Zusammenfassend lässt sich also sagen, dass das Transferstärke-Coaching wirksam war. Die Teilnehmerinnen und Teilnehmer der Experimentalgruppe haben ihre Transferstärke signifikant erhöhen können, wie sich auch aus den Mittelwerten der verschiedenen Testzeitpunkte gut ablesen lässt.

Allerdings ist zu berücksichtigen, dass diese Studie einige Limitationen mit Blick auf die Stichprobenselektion aufweist. Zum einen erfolgte die Zuordnung zu den Versuchsgruppen freiwillig und nicht randomisiert. Allein die Motivation zur Teilnahme am Transferstärke-Coaching könnte hier positiv eingewirkt haben. Genauso ist zu erwähnen, dass es eine kleine Stichprobe war, bei der außerdem noch die beiden Versuchsgruppen nicht gleich groß waren. Des Weiteren besteht die Stichprobe zu 85 Prozent aus Frauen. Und schließlich handelte es sich bei den Teilnehmern und Teilnehmerinnen um Master-Wirtschaftspsychologie-Studierende, die aufgrund ihres Studiums dem Thema gegenüber aufgeschlossener gewesen sein könnten als andere Personenkreise.

Insgesamt lässt sich auf der Basis dieser Studie und eigener Trainings der folgende Nutzen aus Teilnehmersicht festhalten: Teilnehmer und Teilnehmerinnen 
finden die Standortbestimmung mithilfe der Transferstärke-Analyse nützlich, da ihnen meistens zwar bewusst ist, dass ihnen die Umsetzung von Lern- und Veränderungsimpulsen in der Praxis nicht so gut gelingt, sie aber nicht wissen, was sie genau dagegen tun können.

Die Transferstärke-Analyse zeigt nun schnell und präzise auf, wo Teilnehmerinnen und Teilnehmer aktiv ansetzen können, um ihren Lern- und Transfererfolg zu steigern. Gerade bei transferschwachen Teilnehmern und Teilnehmerinnen ist das eigene Transferstärke-Profil ein Anstoß für Veränderung, da sie schwarz auf weiß lesen, welche Risiken sie für den Lernerfolg haben. Das sorgt vielfach für emotionale Betroffenheit und den Wunsch, an diesem Thema $\mathrm{zu}$ arbeiten. Dies gilt besonders für Menschen, die an persönlicher Weiterentwicklung interessiert sind.

Durch die Handlungsempfehlungen im Auswertungsbericht und das Transferstärke-Coaching können diese Risiken mit einem vergleichsweise überschaubaren Zeitaufwand überwunden werden. Gerade die Systematik mit Gesprächskontakten und die damit verbundene Notwendigkeit, sich immer wieder mit dem eigenen Lernprozess auseinanderzusetzen, sind maßgeblich für die erzielten Effekte und stellen aus Sicht der Teilnehmerinnen und Teilnehmer einen hohen Nutzwert dar.

\section{Zertifizierung für das Transferstärke-Coaching}

Damit Interessierte den neuen Ansatz des Transferstärke-Coachings selbst nutzen können, braucht es eine Zertifizierung beim Autor dieses Beitrags. Sie besteht im Wesentlichen aus drei Bestandteilen: Der Teilnehmer, die Teilnehmerin durchläuft ein eigenes Transferstärke-Coaching in dem oben beschriebenen Prozess, führt zwei dokumentierte und begleitete Transferstärke-Coachings mit Übungskandidaten und -kandidatinnen durch und erarbeitet sich das Knowhow in Form von Trainingsblöcken bzw. im Selbststudium. Darüberhinaus gibt es eine Supervison beim ersten realen Einsatz.

Das Zertifizierungsprogramm dauert rund fünf Tage und läuft berufsbegleitend über etwa ein halbes Jahr. Es ist als Einzel- oder Gruppenzertifizierung verfügbar und kostet 2.900 Euro pro Person (zzgl. Mehrwertsteuer). Teilnahmevoraussetzungen sind berufliche Erfahrungen in Personalentwicklung, Training bzw. Coaching sowie entsprechende Qualifikationen.

Ein zertifizierter Transferstärke-Coach ist berechtigt, den Online-Test namens Transferstärke-Analyse beim Autor zu bestellen und zu erwerben. Ein Testzugang kostet 150 Euro (zzgl. Mehrwertsteuer). Dabei gibt es je nach Abnahmemenge auch Staffelpreise. 
Wer Wirkung und Nutzen des Transferstärke-Coachings selbst testen möchte, kann hierzu das preisgünstige Kennenlernangebot zum Preis von 250 Euro (zzgl. MwSt.) buchen. Darin enthalten sind der Transferstärke-Test, Auswertungsbericht, Auswertungsgespräch und ein Folgegespräch.

Darüber hinaus gibt es bereits einen Pool an zertifizierten Transferstärke-Coaches, sodass jeder Interessent, jede Interessentin auch über www. transferstaerke.com die Dienstleistung des Transferstärke-Coachings buchen kann.

\section{Ausblick}

Die bisherige Anwendung des Transferstärke-Coaching-Konzepts hat gezeigt, dass Teilnehmerinnen und Teilnehmer in dem beschriebenen Prozess sehr gut sensibilisiert und trainiert werden können, wie sie ihren Lerntransfer grundsätzlich fördern können. In weiteren Studien gilt es nun, vertiefend zu erkunden, in welchen Trainingsformaten (z. B. im Rahmen von Intervalltrainingsprogrammen) und bei welchen Zielgruppen das Transferstärke-Coaching am besten zum Einsatz kommen kann. Eigene praktische Erfahrungen legen nahe, dass sich der größte Anwendungsnutzen im Bereich des Talentmanagements bzw. bei Leadership-Development-Programmen ergibt. Mit dem Transferstärke-Coaching lassen sich eng umrissene Entwicklungsthemen sehr effizient bearbeiten. Hinzu kommt, dass da hoch motivierte Mitarbeiterinnen und Mitarbeiter an persönlicher Entwicklung sehr interessiert sind. Die Firmen investieren zudem viel Geld in ihre High Potentials und wollen demnach auch einen hohen Umsetzungsgrad des Gelernten erreichen. Darüber hinaus werden diese Personen im Lauf ihrer Karriere selbst Mitarbeitende führen und entwickeln. Das erworbene Transferstärke-Know-how ist demnach ein wichtiger Baustein für die eigene Führungspraxis.

Eine weitere Fragestellung betrifft den Mehrwert des Transferstärke-Coachings gegenüber vergleichbaren Ansätzen: Hier sind zu nennen: das herkömmliche Coaching oder bereits bestehende Formate des Transfer-Coachings (Behrendt, 2004; Behrendt, Pritschow \& Rüdesheim, 2007; Alke, 2010), das virtuelle Transfercoaching (Geißler, 2010; Geißler, 2011; Geißler, Hasenbein \& Wegener, 2013) oder das Telefon-Shadowing (Leder, 2013). 


\section{Literatur}

Alke, M. (2010). Mit Anstoß zur Veränderung. Transfercoaching. managerSeminare, 146 (Mai), 58-63.

Baldwin, T. T., Ford, J. K. (1988). Transfer of training: a review and directions for future research. Personnel Psychology, 41 (1), 63-105.

Behrendt, P. (2004). Wirkfaktoren im Psychodrama und Transfercoaching. Diplomarbeit. Institut für Psychologie, Albert-Ludwigs-Universität Freiburg.

Behrendt, P. (2012). Freiburger Erfolgsfaktoren-Coaching. Organisationsberatung, Supervision, Coaching, 19 (4), S. 391-404.

Behrendt, P., Pritschow, K., Rüdesheim, B. (2007). Transfercoaching. Vom Seminar zur erfolgreichen Umsetzung im Berufsalltag. Zeitschrift für Führung und Organisation (zfo), 76 (1), 49-56.

Bergel, S. (2008). Semantik trifft E-Learning - Der Trend zum Wissenshäppchen. managerSeminare, 124 (Juli), 20-24.

Bühner, M. (2011). Einführung in die Test- und Fragebogenkonstruktion (3. Aufl.). München: Pearson.

Chiaburu, D. S., Lindsay, D. R. (2008). Can do or will do? The importance of self-efficacy and instrumentality for training transfer. Human Resource Development International, 11 (2), 199-206.

Diesner, I., Seufert, S. (2010). Trendstudie 2010. Herausforderungen für das Bildungsmanagement in Unternehmen. St. Gallen: Institut für Wirtschaftspädagogik, Universität St. Gallen.

Diesner, I., Seufert, S. (2013). Trendstudie 2012. Herausforderungen für das Bildungsmanagement in Unternehmen. Management Summary. St. Gallen: Swiss Centre for Innovations in Learning, Universität St. Gallen.

Eckelt, A., Sauter, W. (2016). Deutsche Bahn auf dem Weg zur »Bildung 4.0«. wirtschaft + weiterbildung, (1), 36-39.

Erpenbeck, J., Sauter, W. (2013). So werden wir lernen! Kompetenzentwicklung in einer Welt fühlender Computer, kluger Wolken und sinnsuchender Netze. Berlin u. Heidelberg: Springer Gabler.

Euler, D., Seufert, S. (2006). SCIL-Trendstudie. Ergebnisse einer Delphi-Befragung zu den Herausforderungen für das Bildungsmanagement in Unternehmen. SCIL-Arbeitsbericht 9. Januar 2006. St. Gallen: Swiss Centre for Innovations in Learning, Universität St. Gallen.

Fandel-Meyer, T., Schneider, C. (2015). 5. SCIL-Trendstudie 2015/2016. Trends in Corporate Learning? Management Summary. St. Gallen: Swiss Centre for Innovations in Learning, Universität St. Gallen.

Furtner, M. R., Sachse, P. (2011). Self-Leadership-Training. Wirksamkeitsprüfung mit qualitativ-quantitativer Methodenkombination. Wirtschaftspsychologie, 13 (2), 102-111.

Geißler, H. (2010). Gelerntes nachhaltig anwenden. Beispiel: Virtuelles Transfercoaching (VTC). Weiterbildung, (11), 24-26.

Geißler, H. (2011). Coaching meets Training - zur Lösung des Transferproblems durch "virtuelles Transfercoaching (VTC)«. In M. Loebbert, A. Fritze, R. Wegener (Hrsg.), Coaching entwickeln. Forschung und Praxis im Dialog (S. 123-134). Wiesbaden: VS Verlag für Sozialwissenschaften.

Geißler, H., Hasenbein, M., Wegener, R. (2013). E-Coaching: Prozess- und Ergebnisevaluation des »Virtuellen Zielerreichungscoachings«. Organisationsberatung, Supervision, Coaching, 20 (2), 125-142.

Gerathewohl, F. (2015). Optimierung des Lernerfolgs im Rahmen von E-Learning-Formaten. Eine Evaluationsstudie zu Wirksamkeit und Nutzen der Transferstärke-Methode. Bachelorarbeit. Hochschule für angewandtes Management, Erding.

Gillies, C. (2013a). Kleine Portionen, bitte! Microlearning. managerSeminare, 183 (Juni), 56-60.

Gillies, C. (2013b). Die Ära App kommt. Mobile Learning. managerSeminare, 186 (September), 52-56.

Gillies, C. (2014). Schlauer Dreh. Lernvideos. managerSeminare, 201 (Dezember), 62-67. 
Gloger, A. (2009). Die Weiterbildungsminis kommen. Lerntrend Kürze. managerSeminare 132 (März), 56-61.

Gollwitzer, P. M., Sheeran, P. (2006). Implementation intentions and goal achievement: A meta-analysis of effects and processes. Advances in Experimental Social Psychology, 38, 69-119.

Graf, J. (2015). WeiterbildungsSzene Deutschland. Themen und Trends in Training, Beratung, Coaching. Bonn: managerSeminare.

Greif, S., Schmidt, F., Thamm, A. (2012). Warum und wodurch Coaching wirkt. Organisationsberatung, Supervision, Coaching, 19 (4), 375-390.

Gris, R. (2008). Die Weiterbildungslüge. Warum Seminare und Trainings Kapital vernichten und Karrieren knicken. Frankfurt a. M.: Campus.

Grossman, R., Salas, E. (2011). The transfer of training: what really matters. International Journal of Training an Development, 15 (2), 103-120.

Koch, A. (2012). Die Transferstärke-Methode - Neue Wege für eine rentable Personalentwicklung. In R. Reinhardt (Hrsg.), Wirtschaftspsychologie und Organisationserfolg. Tagungsband zur 16. Fachtagung der Gesellschaft für angewandte Wirtschaftspsychologie, Stuttgart, 11. bis 12. Februar 2011 (S. 67-80). Lengerich: Pabst.

Kreutz, B. (2012). Überprüfung der Validität der Transferstärke-Analyse. Eine Validierungsstudie mit ausgewählten Skalen des Inventars sozialer Kompetenzen (IKS). 2. Praxisbericht. SRH Fernhochschule Riedlingen.

Leder, S. (2013). Telefon-Shadowing. Wirksamkeit einer neuen Coaching-Methode zur Umsetzung von Verhaltensänderungen. Diplomarbeit. Abteilung für Psychologie, Arbeits- und Organisationspsychologie, Fakultät für Psychologie und Sportwissenschaften, Universität Bielefeld.

Lienert, G. A., Raatz, U. (1989). Testaufbau und Testanalyse (4., neu ausgest. Aufl.). Weinheim: Beltz.

Marlatt, G. A., Gordon, J. R. (1985). Relapse Prevention. New York: Guilford.

Martens, A. (2015). Gespräch mit dem inneren Du. Trendmethode Selbstcoaching. managerSeminare, 203, (Februar), 56-61.

Marx, R. D. (1982). Relapse Prevention for Managerial Training: A Model for Maintenance of Behavior Change. Academy of Management Review, 7 (3), 433-441.

Marx, R. D. (1993). Improving Management Development Through Relapse Prevention Strategies. Journal of Management Development, 5 (2), 27-40.

Mummendey, H. D., Grau, I. (2008). Die Fragebogenmethode (5. Aufl.). Göttingen: Hogrefe.

Oettingen, G., Gollwitzer, P. M. (2010). Strategies of Setting and Implementing Goals. Mental Contrasting and Implementation Intentions. In J. E. Maddux, J. P. Tangney (Eds.), Social psychological foundations of clinical psychology (pp. 114-135). New York: Guilford Press.

Pachner, A. (2009). Entwicklung und Förderung von selbst gesteuertem Lernen in Blended-Learning-Umgebungen. Eine Interventionsstudie zum Vergleich von Lernstrategietraining und Lerntagebuch. Münster: Waxmann (Empirische Erziehungswissenschaft, Bd. 18).

Pankow, S. (2013). Überprüfung der Konstruktvalidität und Reliabilität sowie Retestreliabilität der Transferstärke unter Berücksichtigung der diskriminanten/konvergenten Validität mit den Konstrukten NEO-FFI und Learning Style Orientation. Bachelorarbeit. Lehrstuhl für Wirtschafts- und Organisationspsychologie, Fakultät für Ingenieurwissenschaften, Universität Duisburg-Essen.

Pape, K.-H. (2015). DB Training will nicht mehr unterrichten. Blog vom 24. April 2015. https:// khpape.wordpress.com/2015/04/24/db-training-will-nicht-mehr-unterrichten/[15.2.2017].

Papenhoff, T. (2013). Der Einfluss einer Verhandlungsschulung auf den Verhandlungsstil im Einkauf unter Berücksichtigung der Persönlichkeit und Transferstärke. Bachelorarbeit. Lehrstuhl für Wirtschafts- und Organisationspsychologie, Fakultät für Ingenieurwissenschaften, Universität Duisburg-Essen.

Reimann, S. (2015). »Ihr lernt, wir wachsen. « Personalentwicklung bei adidas. managerSeminare, 208 (Juli), 62-66. 
Saks, A. M., Belcourt, M. (2006). An investigation of training activities and transfer of training in organizations. Human Resources Management, 45 (4), 629-648.

Saks, A. M., Salas, E., Lewis, P. (2014). The transfer of training. International Journal of Training and Development, 18 (2), 81-83.

Schürf, S. (2012). Validierung der Transferstärkeanalyse mit Hilfe des Maßnahmen-Erfolgs-Inventar (MEI) bei Teilnehmern von Führungskräfteschulungen der Bayerischen Verwaltungsschule (BVS). Masterarbeit. SRH FernHochschule Riedlingen.

Schwarzer, R., Jerusalem, M. (Hrsg.) (1999). Skalen zur Erfassung von Lehrer- und Schülermerkmalen. Dokumentation der psychometrischen Verfahren im Rahmen der Wissenschaftlichen Begleitung des Modellversuchs Selbstwirksame Schulen. Berlin: Freie Universität Berlin.

Wechsler, T. (2012). Das coachingtool-spezifische Wirkfaktorenmodell. Organisationsberatung, Supervision, Coaching, 19 (4), 405-423.

Weinbauer, I. (2015). Die Verbreitung von Transferpraktiken in Österreich. Eine neo-institutionalistische Betrachtung des Research-Practice-Gaps der Transferforschung. Dissertation. Institut für Human Resource and Changemanagement, Johannes Kepler Universität, Linz. 


\section{Ambivalenzen des Coachings \\ Über nicht intendierte Wirkungen in Zeiten der Selbstoptimierung}

Laura Glauser

Dieser Beitrag fragt nach den Wirkungen von Coaching auf Individuen im Hinblick auf die gesellschaftliche Anforderung, sich als »unternehmerisches Selbstı flexibel und selbstverantwortlich zu managen und zu optimieren. Die Ergebnisse meiner ethnologischen Feldforschung in Career Services von Hochschulen, deren zentrale Methoden teilnehmende Beobachtung und qualitative Interviews waren, zeigen hier eine verstörende Ambivalenz: So wirkt zwar der lösungsorientierte Ansatz im Coaching mit seinem Fokus auf den Ressourcen einer Person und individuellen Lösungsoptionen bestärkend, doch da überindividuelle Faktoren ausgeblendet werden, welche die Gestaltungsmöglichkeiten des Einzelnen begrenzen, werden zugleich Selbstzweifel und Selbstbezichtigungen gefördert. Abschließend folgen erste Überlegungen, wie in der Coaching-Praxis den verunsichernden Wirkungen von Coaching entgegengearbeitet werden kann.

In diesem Beitrag frage ich nach der Wirkung von Coaching auf Individuen vor dem Hintergrund vorherrschender gesellschaftlicher Diskurse und Anforderungen, deren leitende Prinzipien in der sozialwissenschaftlichen Forschung unter dem Begriff des »unternehmerischen Selbst « (Bröckling, 2007) subsumiert worden sind. In diesem Diskurs werden Individuen als »freie« Subjekte adressiert und dazu aufgefordert, eigenverantwortlich zu agieren, aktiv zu sein und sich selbst nach unternehmerischen Prinzipien zu »managen « und zu optimieren. Zentral für diesen Diskurs ist die Idee eines aktiven Subjekts, das gemäß seiner marktkonformen Bestimmung zweckrational handelt, um erfolgreich zu sein. Für diesen Erfolg ist jede und jeder selbst verantwortlich; die Selbstständigkeit mit dem zugrunde liegenden Appell an die Eigenverantwortlichkeit sind weitere zentrale Elemente dieses Diskurses.

Die Praxis des Coachings passt mit Grundhaltung und Herangehensweise gut zu den Anforderungen, die an ein unternehmerisches Selbst gestellt werden, 
und es können wichtige Parallelen erkannt werden (vgl. etwa Duttweiler, 2007, S. 47-76; Fietze, 2016; Maasen, Eberfeld, Eitler \& Tändler, 2011; Traue, 2010). So geht es im Coaching darum, Coachees in ihrer Handlungsautonomie und Eigeninitiative zu fördern und sie dabei zu unterstützen, ihre Ziele eigenverantwortlich zu erreichen.

Die Wirkungen von Coaching auf Individuen, so lautet meine These, zeigen dabei jedoch eine grundlegende Ambivalenz auf. Einerseits orientiert sich Coaching als lösungsorientierte Beratungsform am Individuum und seinen Handlungsmöglichkeiten und verfolgt das Ziel, die Einzelnen in ihrem Selbstbewusstsein zu stärken und Sicherheit zu verleihen. Es zeigte sich, wie Coaching den Menschen ein Gefühl von Ermächtigung (empowerment) vermitteln kann, weil sie ihr berufliches Fortkommen selbst gestalten und ihre Ziele erreichen können. Doch gleichzeitig fördert die einseitige Fokussierung auf die Handlungsoptionen, dass Individuen von der Gesellschaft entkoppelt werden und hierdurch die Verunsicherung zunimmt, weil ihre Möglichkeiten zur Gestaltung von Faktoren, die nicht in ihrer Hand liegen, begrenzt werden.

Die negativen Effekte von Coaching wurden in wirtschaftspsychologischen Studien in den letzten Jahren zunehmend erforscht (vgl. Schermuly, 2016; Schermuly, Schermuly-Haupt, Schölmerich \& Rauterberg, 2014); diese Arbeiten ergänzen bereits vorliegende weitreichende Studien zu den Wirkungen von Coaching (vgl. Theeboom, Beersma \& van Vianen, 2014). Für den folgenden Beitrag wurde im Vergleich zu den genannten Arbeiten ein anderer methodischer Zugang gewählt: Mit einer sozialwissenschaftlichen Perspektive auf Coaching können mögliche verunsichernde Wirkungen von Coaching im Hinblick auf den gesellschaftlichen Kontext analysiert und fokussiert werden.

Dem Beitrag liegen Daten aus meiner ethnologischen Feldforschung zugrunde, die ich von Mai 2009 bis Juli 2010 in Career Services von Hochschulen im Rahmen eines Promotionsprojekts durchgeführt habe (vgl. Glauser, 2016; Glauser, 2012). Diese auch Career Center genannten Beratungsinstitutionen von Hochschulen stellen für Studierende und Hochschulabsolventinnen und -absolventen Beratungs-, Informations- und Weiterbildungsangebote zu beruflichen Themen bereit. Ausgangspunkt meiner Forschung war die Frage, wie Akteure und Akteurinnen in akademischen Career Services mit dem Diskurs des "unternehmerischen Selbst « umgehen. Dabei wurden auch Karriere-CoachingAngebote untersucht (vgl. Glauser, 2016, S. 94-97), die in diesem Beitrag im Vordergrund stehen. Zentrale Methoden waren teilnehmende Beobachtung und qualitative halbstrukturierte Interviews mit vierzehn Studierenden und Hochschulabsolventinnen und -absolventen sowie mit vierzehn Mitarbeiterinnen und Mitarbeitern, von denen einige als Coach tätig waren. Aus ethischen Gründen 
habe ich die beforschten Institutionen sowie die Namen der Gegenüber anonymisiert. Mit der Methode der teilnehmenden Beobachtung partizipierte ich aktiv am Alltag der Menschen innerhalb des Career Service und nahm an dessen Veranstaltungen und Beratungen teil. Während der gesamten Feldforschung führte ich zur Dokumentation meiner informellen Gespräche und Beobachtungen ein Feldforschungstagebuch, im Folgenden mit FT abgekürzt.

\section{Arbeit am erfolgreichen Selbst}

In der Feldforschung haben sich der massive Erfolgsdruck und die vielfältigen Anstrengungen gezeigt, mit denen arbeitssuchende Absolventinnen und Absolventen versuchen, den gesellschaftlichen Anforderungen zu genügen. An der Schwelle zwischen Studium und Berufseinstieg leiden sie darunter, nirgends richtig dazuzugehören. Ihnen allen gemeinsam ist die Angst, keine passende Beschäftigung zu finden, über längere Zeit ohne finanzielle Sicherheit zu sein und keinen Erfolg zu haben. So sagt eine Teilnehmerin in einem Gruppencoaching: "Ich befinde mich im freien Fall auf dem Arbeitsmarkt. Und da ist auch immer wieder die große Angst, durch die Maschen zu fallen, weil ich zu alt bin« (FT 2010). Ihre Empfindung, sich »im freien Fall« zu befinden, verweist auf ihre Haltlosigkeit und ihren Wunsch nach sozialer Einbindung. Zudem deutet sie auf ihre Ohnmacht hin; sie hat das Gefühl, von der Gunst Dritter abhängig zu sein und ihre berufliche Situation nicht aus eigener Kraft verändern zu können. Nicht zuletzt macht die Formulierung ihre Angst, sozial abzusteigen, deutlich; sie beschreibt ihre Furcht, nicht aufgefangen zu werden und außerhalb der erfolgreichen Berufswelt zu bleiben.

Auffällig ist, dass arbeitssuchende Hochschulabsolventinnen und -absolventen oft den Eindruck haben, nicht zu genügen, wie es eine Gesprächspartnerin ausdrückt: »Ich werde das nie schaffen, ich kann mich nicht präsentieren! ... Heutzutage zählt nur noch, dass man sich gut präsentieren kann, und gar nicht, dass man fachlich gut ist « (FT 2009). Zudem schreiben sie sich selbst die Verantwortung für ihre berufliche Lage zu (Neckel \& Wagner, 2014) und machen sich Vorwürfe, zu wenig zu leisten - wie etwa Lena, 25 Jahre alt, die vergeblich versucht, wenigstens ein Volontariat zu bekommen. Im Interview erzählt die Geisteswissenschaftlerin, wie sie die Zeit der Bewerbung empfindet: »bei mir [ist] schon immer auch ganz stark so ein Schuldgefühl da«, sie befürchtet, sich zu selten zu bewerben, und meint: »Ich muss mich immer vor mir selber rechtfertigen, dass ich auch was geschafft habe am Tag« (Interview Lena 2009). 
Oft geht die Arbeitssuche mit Schamgefühlen einher. In den Gesprächen erzählen mir Absolventinnen und Absolventen häufig von ihrer Sorge, sie könnten als »faul« angesehen werden, so wie Martin, 26-jähriger Mathematik-Student: »Das Schlimmste, was ich mir vorstellen könnte, ist, dass die [sein soziales Umfeld] von mir das Bild haben könnten: 'Ja, der versucht es ja gar nicht, der nimmt das auf die leichte Schulter.< [...] Ich habe schon das Gefühl, dass ich denen schon zeigen muss: `He, ich bin aktiv, ich lasse das nicht treiben « (Interview Martin 2010). Seit einem Jahr sucht er eine Anstellung, wobei er aufgrund seiner erfolglosen Bemühungen das ständige Bedürfnis verspürt, seiner Umgebung zu zeigen, wie sehr er sich wirklich anstrengt, eine Arbeit zu finden.

In solch unsicheren Übergangssituationen nach dem Studium ist die Figur des "unternehmerischen Selbst " mit ihrem Appell, den Erfolg in die eigene Hand zu nehmen, besonders ansprechend, verspricht sie doch in einer ohnmächtigen Lage den Gewinn von Erfolg bringender Autonomie. Die Studierenden versuchen, den Anforderungen dieser Figur zu entsprechen, um sich sicherer zu fühlen und eine realistische Hoffnung auf die selbstbestimmte Lösung ihrer beruflichen Krisensituation zu gewinnen. So sagt Martin: »Ich möchte auch gerne endlich mal was selber in die Hand nehmen und selber etwas entscheiden und etwas anfangen " (Interview Martin 2010). Sie möchten das Gefühl haben, aktiv zu sein, und bemühen sich, an ihren Qualifikationen zu arbeiten, sich besser zu organisieren und ihren Lebenslauf zu optimieren, wofür sie sich permanent hinterfragen und überprüfen. Mit der Hoffnung auf beruflichen Erfolg suchen sie auch in den Coaching-Angeboten der Career Services Rat und Orientierung.

\section{Der Umgang mit den gesellschaftlichen Anforderungen im Coaching}

Die Beraterinnen und Berater der Career Services versuchen auch im Rahmen von Einzel- und Gruppencoachings, Rat suchende Personen in ihrer beruflichen Orientierung und Karriereplanung zu unterstützen. Sie sind tagtäglich mit deren Unsicherheit und Existenzängsten konfrontiert und sehen es als ihre Aufgabe, den Ratsuchenden Mut zu machen und ihnen Zuspruch zu geben.

So sagt eine Beraterin im Interview: »Mir macht es Spaß, mit Menschen zu arbeiten, dass sie in ihre Gestaltungsmacht kommen. Sie dabei zu unterstützen, das macht mir Spaß, ihnen Möglichkeitsräume anzubieten « (Interview Graf 2009). Ihr bereitet es Freude, Menschen auf ihrem beruflichen Weg »einen kleinen Kick [zu] geben« (ebd.). Sie sollen sich über ihre eigene Wirkmacht bewusst 
werden. Damit weist ihnen die Beraterin eine aktive und gestaltende Rolle zu, sie möchte ihre Gegenüber motivieren und sie davon überzeugen, dass es in ihrer Macht liegt, die eigene Situation in eine positive Richtung zu verändern.

Den Hochschulabsolventinnen und -absolventen die eigenen Einflussmöglichkeiten zu verdeutlichen, ihnen Mut zu machen und sie dazu aufzufordern, »ihr Leben selbst in die Hand zu nehmen« (Interview Schröder 2009), darin sehen die Mitarbeiterinnen und Mitarbeiter ihren Auftrag. Eine Mitarbeiterin formuliert es in einem Interview folgendermaßen: »Das ist genau die Kompetenz, die man fördern, aber auch fordern sollte: dass Absolventen selber wissen, was sie in einem bestimmten Moment brauchen, um sich selber gut auf den Weg zu bringen " (Interview Koch 2009). Menschen sollen durch die Unterstützung von außen in die Lage versetzt und gleichzeitig dazu aufgefordert werden, ihre Situation zu verbessern. Sich selbst zu helfen, sieht sie als eine Kompetenz an, die sie sowohl »fördern « als auch »fordern « möchte (ebd.), eine Formulierung, die seit der rot-grünen Regierung unter Bundeskanzler Gerhard Schröder (1998-2005) die Sozialpolitik in Deutschland bis heute dominiert. Gemeinsam mit den Ratsuchenden erarbeiten die Beraterinnen und Berater in Career Services Techniken, wie sich diese besser bewerben und managen können, um Sicherheit, Erfolg und berufliche Zufriedenheit zu erlangen.

Bei den Coaching-Angeboten fällt auf, dass die Beraterinnen und Berater die individuellen Gestaltungsmöglichkeiten und Chancen thematisieren, während die Grenzen der Handlungsmöglichkeiten und sozial bedingte Erfahrungen kaum Raum bekommen. Ängste, Ohnmachtsgefühle und überindividuelle Gründe von beruflicher Erfolglosigkeit werden damit ausgeblendet. Hier zeigt sich eine Gefahr des lösungsorientierten Beratungsansatzes im Coaching mit den Schwerpunkten auf den Ressourcen einer Person und auf mögliche Lösungen. Die lösungsorientierten Interventionen setzen auf die Kraft einer positiven Perspektive, um so die "Stärken zu stärken «, wie es die Beraterinnen und Berater selbst nennen (Interview Graf 2009). Mit der Intention, das Individuum zu stärken, damit es seine Ziele erreichen und seine Möglichkeiten verbessern kann, gehen sie strukturell weniger auf die Analyse der Ursachen von beruflichem Misserfolg ein (Duttweiler, 2007, S. 27).

Dieser Zugang ist auch vor dem Hintergrund der geringen personellen und finanziellen Ressourcen der Career-Service-Institutionen von Hochschulen zu sehen. Im Grunde lassen diese keine lang andauernden und vertieften Coaching-Prozesse zu, es werden nur ein bis zwei einstündige Einzelsitzungen oder zeitlich auf wenige Stunden begrenzte Gruppenberatungen angeboten. Solche Formate geben Coaches kaum die Möglichkeit, vertieft auf die individuelle Situation der Menschen und überindividuelle Faktoren des beruflichen Erfolgs 
einzugehen. Insofern drängt die personelle und finanzielle Ausstattung solcher Beratungsinstitutionen, neben der inhaltlichen Bevorzugung des lösungsorientierten Ansatzes, auf die Wahrnehmung der Stärken von Individuen und nicht auf ihre Ohnmacht oder überindividuelle Faktoren.

\section{Die ambivalente Wirkung von Coaching}

Dieser Fokus auf die Selbstwirksamkeit und die Handlungsmöglichkeiten im Coaching hat eine ambivalente Wirkung auf die Individuen. Einerseits konnte ich feststellen, dass sich die Ratsuchenden durch die Beratungen unterstützt fühlen und für sich neue Optionen und Chancen erkennen. Sie drücken immer wieder die große Dankbarkeit gegenüber den Beratern und Beraterinnen aus. Der Fokus auf die Handlungsoptionen hat eine bestärkende Wirkung und verleiht den Ratsuchenden eine psychologische Sicherheit. Es gibt ihnen das befriedigende Gefühl und die Zuversicht, das eigene berufliche Fortkommen selbst gestalten und ihre berufliche Situation aus eigenen Kräften verbessern zu können.

Andererseits nimmt durch die einseitige Fokussierung der individuellen Möglichkeiten und die Betonung der Selbstverantwortung auch der Erfolgsdruck und die persönliche Verunsicherung zu. Mit der Betonung eines aktiven, selbstverantwortlichen Individuums und seiner Spielräume geraten unter anderem die gesellschaftlichen Bedingungen und Zwänge aus dem Blickfeld. So werden in der Beratung weniger Faktoren diskutiert, die außerhalb des individuellen Einflussbereichs liegen, wie zum Beispiel Glück oder die Tatsache, dass für eine einzige Stelle oft Hunderte von Bewerbungen eingehen. Ausgeblendet bleiben wirkmächtige gesellschaftliche Faktoren wie zum Beispiel Beziehungsreichtum und materieller Besitz, auch wenn insbesondere die Elitenforschung zeigt, wie sehr beruflicher Erfolg auch von »ererbten« Beziehungen oder vom Habitus abhängig ist (Hartmann \& Kopp, 2001).

Ein solches Ausblenden von wichtigen überindividuellen Faktoren hat zur Folge, dass die Absolventen und Absolventinnen Gründe für ihre beruflichen Probleme, Misserfolge oder gar für ihr Scheitern nur bei sich suchen und permanent auf sich selbst zurückgeworfen werden. Sie haben das Gefühl, den Anforderungen der Arbeitswelt nicht zu genügen, und machen sich selbst verantwortlich, wenn sie nicht gleich eine Arbeitsstelle finden. Sie plagt ein schlechtes Gewissen, zu wenig aktiv zu sein, trotz ihren vielfältigen und intensiven Bemühungen. Geleitet von der Idee eines selbstverantwortlichen Individuums und konfrontiert mit neoliberalen Diskursen, die die Arbeitslosigkeit letztlich 
auf eigenes Verschulden zurückführen, geben sie sich oft selbst die Schuld für ihren mangelnden Erfolg und leiden an Versagensgefühlen - das Ziel von Coaching, Menschen zu stärken, wird also konterkariert.

\section{Ausblicke auf eine gesellschaftlich bewusste Coaching-Praxis}

Die Ergebnisse meiner Feldforschung werfen eine wichtige Frage für die Praxis des Coachings auf: Wie ist mit gesellschaftlich dominanten Appellen nach Selbstoptimierung umzugehen, wenn damit nicht Erfolgsdruck und Selbstzweifel bei den Individuen kontraproduktiv verstärkt werden soll?

Die unter dem Begriff des unternehmerischen Selbst gefassten Wertvorstellungen, wie sein Glück selbst in die Hand nehmen, selbst aktiv werden und eigenverantwortlich agieren, sind auch im lösungsorientierten Coaching leitend und haben eine widersprüchliche Wirkung. Einerseits werden sie verstanden als Aufforderungen, selbstbestimmt und selbstbewusst zu agieren, sich nicht unterzuordnen und die eigene Individualität möglichst gut zu entfalten. Anderseits befördern solche Grundhaltungen der Selbstbestimmung und Eigenverantwortlichkeit auch Versagens- und Ohnmachtsgefühle, wenn der Erfolg ausbleibt.

Aus diesem Grund sehe ich das selbstreflexive und kritische Hinterfragen dieser gesellschaftlichen Werte und Normen im Rahmen des Coachings als sehr wichtig an, um Gefühlen der Überforderung oder verunsichernden Selbstzweifeln entgegenzuwirken. Coaching-Interventionen wie die der Rückspiegelung oder das zirkuläre Fragen können eingesetzt werden, um einen solchen Reflexionsprozess zu befördern und einen »Reflexionsraum " anzubieten (Fietze, 2016, S. 43). So können sich die Klientinnen und Klienten über ihre eigenen, auch gesellschaftlich bedingten Wertvorstellungen und Ansprüche an sich selbst bewusst werden und auf diese Weise versuchen, einen Umgang zu finden, der nicht selbstdestruktiv wirkt. Zudem erscheint es als wichtig, die Ängste und Zweifel der Coachees anzuerkennen und in die Coaching-Arbeit zu integrieren. Ferner zeigen die Forschungsergebnisse, wie wichtig es ist, auf die Grenzen der individuellen Handlungsmöglichkeit zu verweisen und das Individuum als Teil eines gesellschaftlichen Umfelds zu reflektieren. Gerade die klassischen systemischen Methoden, die nach dem Kontext des Individuums fragen, nach seinen Begrenzungen und den strukturellen Bedingungen des eigenen Handelns, sind hier hilfreich. Insbesondere für die Arbeit im Bereich von Hochschulen erweisen sich Peer-Coaching-Formate als gut geeignet. Im gegenseitigen Austausch wird für die Einzelnen deutlich, dass die anderen ähnliche Schwierigkeiten haben, was den individuellen Mangel- und Versagensgefühlen 
entgegenwirkt. Auf diese Weise kann Coaching die Sensibilität für die eigenen, auch gesellschaftlich bedingten Grenzen und Zwänge fördern und gleichzeitig versuchen, die Individuen auf dem Weg zu einem realistischen Umgang mit diesen Beschränkungen zu unterstützen.

\section{Literatur}

Bröckling, U. (2007). Das unternehmerische Selbst. Soziologie einer Subjektivierungsform. Frankfurt a. M.: Suhrkamp.

Duttweiler, S. (2007). Sein Glück machen. Arbeit am Glück als neoliberale Regierungstechnologie. Konstanz: UVK.

Fietze, B. (2016). Coaching in der reflexiven Moderne. Zwischen Komplexitätssteigerung und Handlungsfähigkeit. In R. Wegener, S. Deplazes, M. Hasenbein, H. Künzli, A. Ryter, B. Uebelhart (Hrsg.), Coaching als individuelle Antwort auf gesellschaftliche Entwicklungen (S. 36-44). Wiesbaden: Springer VS.

Glauser, L. (2012). Arbeit an der Ware »Ich«: Zum subjektiven Umgang mit dem »unternehmerischen Selbst « in Career Services. In G. Koch, B. J. Warneken (Hrsg.), Wissensarbeit und Arbeitswissen. Zur Ethnografie des kognitiven Kapitalismus (S. 379-393). Frankfurt a. M.: Campus.

Glauser, L. (2016). Das Projekt des unternehmerischen Selbst. Eine Feldforschung in der Coachingzone. Bielefeld: transcript.

Hartmann, M., Kopp, J. (2001). Elitenselektion durch Bildung oder durch Herkunft? Promotion, soziale Herkunft und der Zugang zu Führungspositionen in der deutschen Wirtschaft. Kölner Zeitschrift für Soziologie und Sozialpsychologie, 53 (3), 436-466.

Maasen, S., Eberfeld, J., Eitler, P., Tändler, M. (Hrsg.) (2011). Das beratene Selbst. Zur Genealogie der Therapeutisierung in den "langen "Siebzigern. Bielefeld: transcript.

Neckel, S., Wagner, G. (Hrsg.) (2014). Leistung und Erschöpfung. Burnout in der Wettbewerbsgesellschaft. Berlin: Suhrkamp.

Schermuly, C. C. (2016). Negative Effekte von Coaching für Klienten - Definition, Häufigkeiten, Kategorien und Ursachen. In C. Triebel, J. Heller, B. Hauser, A. Koch (Hrsg.), Qualität im Coaching (S. 205-214). Berlin: Springer.

Schermuly, C. C., Schermuly-Haupt, M.-L., Schölmerich, F., Rauterberg, H. (2014). Zu Risiken und Nebenwirkungen lesen Sie ... Negative Effekte von Coaching. Zeitschrift für Arbeits- und Organisationspsychologie, 58 (1), 17-33.

Theeboom, T., Beersma, B., Vianen, A. E. M. van (2014). Does coaching work? A meta-analysis on the effects of coaching on individual level outcomes in an organizational context. The Journal of Positive Psychology, 9 (1), 1-18.

Traue, B. (2010). Das Subjekt der Beratung. Zur Soziologie einer Psycho-Technik. Bielefeld: transcript. 


\title{
Das Impostor-Phänomen - ein Thema im Coaching?
}

Eva Traut-Mattausch und Mirjam Zanchetta

\begin{abstract}
Vom Impostor-Phänomen sprechen wir dann, wenn jemand sich innerlich, trotz objektiver Beweise für das Gegenteil, als intellektuellen und professionellen Hochstapler erlebt. Betroffene glauben, anderen ihre Kompetenz nur vorzuspielen, und leiden daher unter ständiger Angst, als weniger leistungsstark entlarvt zu werden. Das Phänomen kann auch im Coaching Thema sein. In diesem Beitrag wird es mitsamt seinen negativen Konsequenzen im persönlichen und wirtschaftlichen Bereich beschrieben. Im Anschluss werden mögliche Ansätze zur Bearbeitung im Coaching vorgestellt.
\end{abstract}

Nicht selten kommen Personen ins Coaching, die davon berichten, sie fühlten sich durch Personen in ihrem beruflichen Umfeld überschätzt. Werden sie von ihrer Führungskraft für den erfolgreichen Projektabschluss gelobt, so denken sie, sie hätten nur Glück gehabt, dass alles gut gelaufen sei. Wenn Sie das Angebot erhalten, eine höhere Position zu übernehmen, haben sie das Gefühl, dies geschehe nur, weil sie zur rechten Zeit am rechten Ort gewesen seien oder zufällig die richtigen Leute kennen würden. Sie selbst halten sich nicht für die Leistungsträgerinnen und Leistungsträger, als die sie gesehen werden.

Solches Erleben wird in der Literatur als Impostor-Phänomen beschrieben. Es tritt besonders bei Personen mit objektivem akademischem und/oder beruflichem Erfolg auf und zeichnet sich durch eine Attribution der Leistungen an äußerliche Ursachen aus (Clance \& Imes, 1978). Leistungen und Erfolge werden nicht auf stabile Fähigkeiten zurückgeführt, Betroffene halten sich für inkompetent und denken, sie hätten bei Beförderungen oder positiven Rückmeldungen einfach nur Glück gehabt. Sie halten sich, völlig zu Unrecht, für Hochstapler und leiden ständig an Angst, ihre vermeintliche Hochstapelei bzw. ihre Unfähigkeit könnte erkannt werden (ebd.). Dieses Gefühl der Hochstapelei und die damit einhergehende Angst, entlarvt zu werden, kann sowohl ein schnelles Ende der Karriere (durch Vermeiden von Herausforderungen) als auch psychische Beschwerden nach sich ziehen. Aufgrund der Annahme, dass bis zu siebzig Prozent der Menschen aus allen Gesellschaftsschichten betroffen sein können (Gravois, 
2007) - in geringerem oder höherem Ausmaß -, kann das Impostor-Phänomen ein Thema sein, das vielen Coaches mit ihren Coachees begegnet.

\section{Theoretischer und empirischer Hintergrund}

Clance und Imes (1978) arbeiteten mit mehr als 150 Frauen (u. a. mit qualitativen Interviews im therapeutischen Setting), die über Doktortitel in unterschiedlichen Bereichen verfügten, bekannte Expertinnen in ihrem Feld waren oder Studentinnen, die durch ihre akademische Exzellenz Aufsehen erregt hatten (ebd.). Trotz erworbenen akademischen Graden, trotz Lob durch Vorgesetzte usw. fühlten sie sich nicht erfolgreich. Sie empfanden sich als Hochstaplerinnen, die es geschafft hatten, andere zu täuschen (Klinkhammer \& Saul-Soprun, 2009). Daraus resultierte bei jeder zukünftigen Leistungssituation die Angst, enttarnt zu werden - die Furcht, dass jemand dahinterkommen könnte, wie inkompetent sie tatsächlich seien. Clance und Imes (1978) erklärten sich das Auftreten des Impostor-Phänomens auf Basis von Befunden der Attributionstheorie, wonach Frauen und Männer Erfolge unterschiedlich attribuieren. Mit Bezug auf ein Review von Deaux (1976) zu den Geschlechterunterschieden bei Attributionsprozessen sahen sie die Parallelen darin, dass Frauen mit geringeren Erwartungen - anders als Männer - Erfolge (also für sie unerwartete Ereignisse) external und temporär attribuieren (Glück, Anstrengung). Versagen attribuieren Frauen hingegen internal und stabil (fehlende Kompetenz), während Männer ihre Misserfolge mit Pech oder der Schwierigkeit der Aufgabe erklären, also external und temporär. Frühere empirische Untersuchungen wiesen das damit verbundene verstärkte Auftreten des Impostor-Phänomens bei Frauen nach (z. B. Henning, Ey \& Shaw, 1998; King \& Cooley, 1995). Aktuellere Studien berichten von ähnlich häufigem Auftreten bei Männern (z. B. Bechtoldt, 2015; Cokley et al., 2015; Crawford, Shanine, Whitman \& Kacmar, 2016; Rohrmann, Bechtoldt \& Leonhardt, 2016).

\section{Welche Konsequenzen kann das Auftreten des Impostor-Phänomens haben?}

Als man begann, das Impostor-Phänomen zu studieren, wurden in erster Linie die Konsequenzen im persönlichen bzw. klinischen Kontext untersucht. Dabei wurde ein geringeres Wohlbefinden und das Gefühl, wenig meistern zu können (September, McCarrey, Baranowsky, Parent \& Schindler, 2001), sowie erhöhter psychologischer Disstress (Henning et al., 1998) festgestellt. Es zeigt sich zudem, dass bei Impostoren das Risiko, (soziale) Angststörungen (Bernard, Dollinger \& 
Ramaniah, 2002; Chrisman, Pieper, Clance, Holland \& Glickauf-Hughes, 1995; Henning et al., 1998; Oriel, Plane \& Mundt, 2004; Thompson, Davis \& Davidson, 1998) oder Depressionen zu entwickeln (Bernard et al., 2002; Chrisman et al., 1995; Henning et al., 1998; McGregor, Gee \& Posey, 2008; Oriel et al., 2004; Thompson et al., 1998), erhöht ist.

In den letzten fünf Jahren wurden insbesondere die Konsequenzen im wirtschaftlichen Kontext betrachtet (Bechtoldt, 2015; Grubb \& McDowell, 2012; Jöstl, Bergsmann, Lüftenegger, Schober \& Spiel, 2012; McDowell, Grubb \& Geho, 2015; Neureiter \& Traut-Mattausch, 2016a, 2016b; Vergauwe, Wille, Feys, De Fruyt \& Anseel, 2015). Beispielsweise stellten Studien einen negativen Zusammenhang fest zwischen dem Impostor-Phänomen auf der einen Seite und auf der andern der beruflichen Selbstwirksamkeitsüberzeugung (Jöstl et al., 2012; McDowell et al., 2015; Neureiter \& Traut-Mattausch, 2017), dem Organizational Citizenship Behavior (OCB) ${ }^{1}$, dem affektiven Commitment, der Arbeitszufriedenheit und wahrgenommenen organisationalen Unterstützung (Grubb \& McDowell, 2012; McDowell et al., 2015; Vergauwe et al., 2015). Eine Untersuchung mit Führungskräften und Managern zeigte zum Beispiel, dass das Erleben des Impostor-Phänomens und eine negative Sicht auf sich selbst Auswirkungen auf das Delegationsverhalten der Betroffenen hat (Bechtoldt, 2015). Weiter berichteten Personen mit Impostor-Gefühlen von weniger Karriereplanung, einem geringeren Karrierestreben, einer geringeren Motivation, eine Führungsrolle zu übernehmen (Neureiter \& Traut-Mattausch, 2016a), und erhöhten Karriere-Entscheidungsschwierigkeiten (Neureiter \& Traut-Mattausch, 2017).

\section{Wie lässt sich das Impostor-Phänomen erfassen?}

Um feststellen zu können, in welchem Ausmaß Coachees vom Impostor-Phänomen betroffen sind, kann ein Erhebungsfragebogen eingesetzt werden. Dazu füllen die Coachees die deutsche Clance Impostor Phenomenon Scale (GCIPS) aus (Brauer \& Wolf, 2016; Clance, 1985). Der Erhebungsfragebogen besteht aus zwanzig Aussagen, die auf einer fünfstufigen Skala ( 1 = überhaupt nicht zutreffend, $2=$ selten, $3=$ manchmal, $4=$ oft, $5=$ absolut zutreffend) zu bewerten sind. Beispielaussagen sind: »Ich kann den Eindruck erwecken, kompetenter zu

1 Freiwilliges Verhalten, das sich positiv auf die Funktionsfähigkeit der Organisation auswirkt und im Rahmen des formalen Anreizsystems nicht direkt oder explizit berücksichtigt wird (vgl. Nerdinger, Blickle \& Schaper, 2008, S. 575). 
sein, als ich bin.« Oder: »Ich habe Angst, dass Leute, die mir wichtig sind, herausbekommen, dass ich nicht so fähig bin, wie sie glauben. « Durch die Addition der Punkte erhält man einen Summenwert, der, dividiert durch die Anzahl der Fragen, den Mittelwert ergibt. Je höher der resultierende Wert ist, desto stärker ist der Coachee vom Impostor-Phänomen betroffen.

\section{Interventionsansätze beim Impostor-Phänomen}

Bisher wurden vor allem therapeutische Ansätze diskutiert - zum Beispiel therapeutisch angeleitete Selbsthilfegruppen (Clance \& Imes, 1978) oder multimodale Therapien mit Elementen der Gestalttherapie (Clance, Dingman, Reviere \& Stober, 1995). Klinkhammer und Saul-Soprun (2009) haben erstmals vorgeschlagen, Personen, die unter dem Impostor-Phänomen leiden, mit Coaching und Supervision zu unterstützen. Diese Idee möchten wir aufgreifen und folgendes Konzept für ein Coaching von Impostoren vorschlagen:

1. Klinkhammer und Saul-Soprun (2009) betonen die Wichtigkeit der Aufklärung der Betroffenen über das Impostor-Phänomen. Der Coachee sollte deshalb zu Beginn eines Impostor-Coachings über das Phänomen im Allgemeinen und die damit verbundenen Aspekte im Besonderen aufgeklärt werden. Diese Aufklärung bzw. Psychoedukation kann sowohl mündlich als auch schriftlich oder durch den Einsatz von audiovisuellen Medien erfolgen. Eine längsschnittartig angelegte Studie von Scheibling (2015) zeigte beispielsweise, dass die Betrachtung eines Aufklärungsfilms zu einem signifikanten Wissenszuwachs über das Impostor-Phänomen führt.

2. Klinkhammer und Saul-Soprun (2009) führen weiter die ressourcen- und lösungsorientierte Beratung an und empfehlen den Einsatz der »Säulen der Identität" (Petzold, 1993), da dadurch der Fokus auf die Ressourcen des Coachee gelegt werden kann. Der Coach kann die Identitätssäulen (Leiblichkeit, soziale Beziehungen, Arbeit und Leistung/Freizeit sowie materielle Sicherheiten und Werte) als diagnostisches Instrument zur Einschätzung der persönlichen Stabilität des Coachee im Gesamten oder auch bezogen auf spezifische Teilbereiche einsetzen (Petzold, 2012). Petzold beschreibt, dass die Exploration der Säulen sowohl durch den Einsatz eines Fragebogens (FESI; vgl. Kames, 1992) als auch über bildhafte Darstellungen (Müller \& Petzold, 1998) erfolgen kann. Für Coachees, die vom Impostor-Phänomen betroffen und sich dadurch ihrer Ressourcen nicht in vollem Umfang bewusst sind, empfehlen wir, Identitätsbilder anzufertigen (Petzold, 2012; Petzold \& Orth, 1994). Durch solche Bilder können Ressourcen mit Farben, Formen 
und Symbolen oder figürlichen Elementen visualisiert und damit leichter zugänglich gemacht werden.

3. Um der Stimme des Impostor-Phänomens weniger Raum zu geben, wird weiter die Arbeit mit dem »Inneren Team « (Schulz von Thun, 1998) empfohlen. Sie zeigte sich bereits bei der Bearbeitung der psychologischen Dimensionen mangelnder Präsentationskompetenz (Funken, 2010) als zielführend. Dabei sollte der Coach darauf achten, dass die hinderlichen Impostor-Stimmen zugunsten der förderlichen Stimmen in den Hintergrund treten, ohne dass der Anspruch besteht, sie völlig zum Verstummen zu bringen. Der Coach kann dem Coachee durch die kritische Betrachtung dieser Stimmen helfen, die inneren Dissonanzen aufzuweichen. Um ein vertieftes Verständnis für die Struktur und die Dynamik des »Inneren Teams« zu erarbeiten, es zu visualisieren, erlebbar zu machen und es zu verändern, bietet sich das Mittel der Aufstellung an (Funken, 2010; König, 2000).

4. Zur Steigerung des Selbstwertgefühls und um sich die eigenen Kompetenzen bewusst zu machen, wird eine Potenzialanalyse (z. B. Rohrschneider, Friedrichs \& Lorenz, 2010) oder ein Kompetenzprofil empfohlen. Rohrschneider et al. (2010) führen als Nutzen für die Ausführenden beispielsweise an, dass sie sich so ein differenziertes Feedback verschaffen können, Klarheit über Stärken und Entwicklungsfelder gewinnen, Entwicklungsunterstützung und auch Anregung zur Selbstreflexion erhalten. Dies kann dazu dienen, die meist durch das Impostor-Phänomen verzerrte Selbsteinschätzung des Coachee mit den Fremdeinschätzungen der Umwelt abzugleichen, zu relativieren und im optimalen Fall zu adaptieren.

5. Mit Rücksicht auf die mit dem Phänomen einhergehende Schamkomponente (Cowman \& Ferrari, 2002) empfehlen wir eine ausgesprochen sensible Herangehensweise. Beispielsweise können Verfahren wie die Potenzialanalyse oder das Kompetenzprofil sehr gut vor dem Hintergrund der Vorbereitung auf eine Bewerbungssituationen in Karriere-Coachings (z. B. Braumandl, Amberger, Falkenberg \& Kauffeld, 2013) eingebettet werden.

Bei der empirischen Überprüfung eines Coachings, bei dem unter anderem mit einer Potenzialanalyse, einem Kompetenzprofil und mit dem »Inneren Team» gearbeitet wurde (die Zusammenstellung der Übungen und die Durchführung erfolgten im Rahmen der Masterarbeit von Anna-Maria Muck [2015]), konnte eine signifikante Reduktion der Impostor-Phänomen-Werte nachgewiesen werden (Zanchetta, Traut-Mattausch, Muck \& Losch, in prep.). Aus diesem Grund empfehlen wir Coaches, denen das Impostor-Phänomen bei ihren Coachees bzw. im Coaching begegnet, die angeführten Verfahren bzw. Methoden anzu- 
wenden. Obwohl inzwischen ein erster empirischer Nachweis für die Wirksamkeit eines Coachings zur Reduktion des Impostor-Phänomens vorliegt, bleibt zu klären, welche Übungen am effektivsten dazu beitragen und somit für ein erfolgreiches Coaching unverzichtbar sind. Ferner sollten unterschiedliche Settings auf ihre Effektivität zur Reduktion von Impostor-Gefühlen hin untersucht werden. Ergänzend zum hier beschriebenen Einzelcoaching könnten auch ein Gruppencoaching oder eine Gruppensupervision hilfreich sein. Möglicherweise stellt ein methodisch angeleiteter Austausch mit anderen Personen einen weiteren Ausweg aus dem destruktiven Impostor-Zirkel dar.

\section{Literatur}

Bechtoldt, M. N. (2015). Wanted: Self-doubting employees - Managers scoring positively on impostorism favor insecure employees in task delegation. Personality and Individual Differences, 86, 482-486. doi:10.1016/j.paid.2015.07.002.

Bernard, N. S., Dollinger, S. J., Ramaniah, N. V. (2002). Applying the big five personality factors to the impostor phenomenon. Journal of Personality Assessment, 78 (2), 321-333. doi:10.1207/ s15327752jpa7802_07.

Brauer, K., Wolf, A. (2016). Validation of the German-language Clance Impostor Phenomenon Scale (GCIPS). Personality and Individual Differences, 102, 153-158.

Braumandl, I., Amberger, B., Falkenberg, F., Kauffeld, S. (2013). Konzept-Coaching. Ausbildung zum Coach für Karriere- und Lebensplanung. Konzept, Theorie und Forschungsresultate. In R. Wegener, A. Fritze, M. Loebbert (Hrsg.), Zur Differenzierung von Handlungsfeldern im Coaching. Die Etablierung neuer Praxisfelder (S. 73-83). Wiesbaden: Springer VS.

Chrisman, S. M., Pieper, W. A., Clance, P. R., Holland, C. L., Glickauf-Hughes, C. (1995). Validation of the clance impostor phenomenon scale. Journal of Personality Assessment, 65 (3), 456-467. doi:10.1207/s15327752jpa6503_6.

Clance, P. R. (1985). The impostor phenomenon: overcoming the fear that haunts your success (pp. 20-22). Atlanta, GA: Peachtree.

Clance, P. R., Dingman, D., Reviere, S. L., Stober, D. R. (1995). Impostor phenomenon in an interpersonal social-context - origins and treatment. Women \& Therapy, 16 (4), 79-96. doi:10.1300/ J015v16n04_07.

Clance, P. R., Imes, S. A. (1978). Impostor phenomenon in high achieving women - Dynamics and therapeutic intervention. Psychotherapy-Theory Research and Practice, 15 (3), 241-247. doi:10.1037/h0086006.

Cokley, K., Awad, G., Smith, L., Jackson, S., Awosogba, O., Hurst, A., Stone, S., Blondeau, L., Roberts, D. (2015). The roles of gender stigma consciousness, impostor phenomenon and academic self-concept in the academic outcomes of women and men. Sex Roles, 73 (9-10), 414-426. doi:10.1007/s11199-015-0516-7.

Cowman, S. E., Ferrari, J. R. (2002). »Am I for real?« Predicting impostor tendencies from self-handicapping and affective components. Social Behavior and Personality: an international journal, 30 (2), 119-125.

Crawford, W. S., Shanine, K. K., Whitman, M. V., Kacmar, K. M. (2016). Examining the impostor phenomenon and work-family conflict. Journal of Managerial Psychology, 31 (2), 375-390.

Funken, I. (2010). Working with the "Inner Team « as an imaginative method in coaching. Organisationsberatung, Supervision, Coaching, 17 (3), 291-301. 
Gravois, J. (2007). You're Not Fooling Anyone. Chronicle of Higher Education, 54 (11), 1 a. A14.

Grubb, W. L., McDowell, W. C. (2012). The impostor phenomenon's impact on citizenship behavior and employee commitment: Flying under the radar. Journal of Business Issues, 1, 1-10.

Henning, K., Ey, S., Shaw, D. (1998). Perfectionism, the impostor phenomenon and psychological adjustment in medical, dental, nursing and pharmacy students. Medical Education, 32 (5), 456-464. doi:10.1046/j.1365-2923.1998.00234.x

Jöstl, G., Bergsmann, E., Lüftenegger, M., Schober, B., Spiel, C. (2012). When will they blow my cover? Zeitschrift für Psychologie, 220 (2), 109-120. doi:10.1027/2151-2604/a000102.

Kames, H. (1992). Ein Fragebogen zur Erfassung der fünf Säulen der Identität (FESI). Integrative Therapie, 4 (92), 363-386.

King, J. E., Cooley, E. L. (1995). Achievement orientation and the impostor phenomenon among college students. Contemporary Educational Psychology, 20 (3), 304-312.

Klinkhammer, M., Saul-Soprun, G. (2009). Das »Hochstaplersyndrom« in der Wissenschaft. Organisationsberatung, Supervision, Coaching, 16 (2), 165-182. doi:10.1007/s11613-009-0120-1.

König, G. (2000). Ein Handwerkszeug für den Coaching-Alltag: Visualisieren und begreifbar visualisieren mit inszenario ${ }^{\circledR}$. In C. Rauen (Hrsg.), Handbuch Coaching (S. 391-396). Göttingen: Hogrefe.

McDowell, W. C., Grubb, W. L., Geho, P. R. (2015). The impact of self-efficacy and perceived organizational support on the imposter phenomenon. American Journal of Management, 15 (3), 23-29.

McGregor, L. N., Gee, D. E., Posey, K. E. (2008). I feel like a fraud and it depresses me: The relation between the imposter phenomenon and depression. Social Behavior and Personality, 36 (1), 43-48. doi:10.2224/sbp.2008.36.1.43.

Muck, A.-M. (2015). No faking anymore! The effectiveness of coaching in the reduction of impostor feelings in comparison to training and waiting-list control group. Masterarbeit, Universität Salzburg.

Müller, L., Petzold, H. (1998). Projektive und semiprojektive Verfahren für die Diagnostik von Störungen, Netzwerken und Komorbidität in der Integrativen Therapie von Kindern und Jugendlichen. Integrative Therapie, 3 (4), 396-438.

Nerdinger, F. W., Blickle, G., Schaper, N. (2008). Arbeits-und Organisationspsychologie. Berlin: Springer.

Neureiter, M., Traut-Mattausch, E. (2016a). An inner barrier to career development: Preconditions of the impostor phenomenon and consequences for career development. Frontiers in Psycho$\log y, 7$, 48. doi:10.3389/fpsyg.2016.00048.

Neureiter, M., Traut-Mattausch, E. (2016b). Inspecting the dangers of feeling like a fake: An empirical investigation of the impostor phenomenon in the world of work. Frontiers in Psychology, 7, 1445. doi:10.3389/fpsyg.2016.01445.

Neureiter, M., Traut-Mattausch, E. (2017). Two sides of the career resources coin: Career adaptability resources and the impostor phenomenon. Journal of Vocational Behavior, 98, 56-69. doi:10.1016/j.jvb.2016.10.002.

Oriel, K., Plane, M. B., Mundt, M. (2004). Family medicine residents and the impostor phenomenon. Family Medicine, 36 (4), 248-252.

Petzold, H. (1993). Integrative Therapie: Klinische Theorie: das biopsychosoziale Modell. Bd. 2. Paderborn: Junfermann.

Petzold, H. (2012). Identität: Ein Kernthema moderner Psychotherapie. Wiesbaden: VS Verlag für Sozialwissenschaften.

Petzold, H. G., Orth, I. (1994). Kreative Persönlichkeitsdiagnostik durch »mediengestützte Techniken« in der Integrativen Therapie und Beratung. Integrative Therapie, 4 (94), 340-391.

Rohrmann, S., Bechtoldt, M., Leonhardt, M. (2016). Validation of the Impostor Phenomenon Among Managers. Frontiers in Psychology, 7, 821.

Rohrschneider, U., Friedrichs, S., Lorenz, M. (2010). Erfolgsfaktor Potenzialanalyse. Wiesbaden: Gabler. 
Scheibling, L. M. (2015). Ist Psychoedukation beim Impostorphänomen eine wirksame Intervention? Masterarbeit, Universität Salzburg.

Schulz von Thun, F. (1998). Miteinander reden 3. Das »innere Team « und situationsgerechte Kommunikation. Reinbek bei Hamburg: Rowohlt.

September, A. N., McCarrey, M., Baranowsky, A., Parent, C., Schindler, D. (2001). The relation between well-being, impostor feelings, and gender role orientation among Canadian university students. Journal of Social Psychology, 141 (2), 218-232.

Thompson, T., Davis, H., Davidson, J. (1998). Attributional and affective responses of impostors to academic success and failure outcomes. Personality and Individual Differences, 25 (2), 381396. doi:10.1016/s0191-8869(98)00065-8.

Vergauwe, J., Wille, B., Feys, M., De Fruyt, F., Anseel, F. (2015). Fear of being exposed: The traitrelatedness of the impostor phenomenon and its relevance in the work context. Journal of Business and Psychology, 30 (3), 565-581. doi:10.1007/s10869-014-9382-5.

Zanchetta, M., Traut-Mattausch, E., Muck, A.-M., Losch, S. (in prep.). Overcoming the impostor phenomenon: The effectiveness of interventions for reducing impostor feelings. 


\section{Effectiveness of Leadership Coaching}

Willem Jan Hofmans

The evaluation of the impact of coaching is lagging behind as demonstrated by the few empirical studies which link coaching to outcomes. This quasi-experimental study on the effectiveness of leadership coaching was completed during a doctoral research project. The contribution of this study is that it is a mixed-methods pre/post longitudinal study. It was approached from the field of business and only professional, certified external coaches were involved. The research used multi-source feedback and reports on multi-level effects, while taking formative evaluations into consideration. Six coaches worked with 30 clients during an average period of 8.7 months to collect data for this project. The results suggest that after leadership coaching there is a significant positive change in clients' mindfulness, a positive, though not statistically significant, improvement in the leadership effectiveness of clients, and a slight, statistically insignificant, improvement in business results. Whereas client satisfaction appears to have a significant impact on the outcome of the coaching, the client-coach relationship and coach satisfaction do not. The achievement of the clients' coaching objectives correlates significantly with leadership competencies, but coaching outcomes do not change significantly enough as a result of achieving objectives.

The world of coaching is a booming business without regulation which everyone can freely enter. According to Theeboom, Beersma and van Vianen (2014), Ely et al. (2010) and De Meuse, Dai and Lee (2009) there is a lack of empirical evidence that coaching delivers the desired results. Organisations for coaches, like the International Coach Federation (ICF) ${ }^{1}$, actively promote the professionalisation of coaching by means of accredited education, certification and research. An opportunity to complete a Doctorate in Business Administration (DBA) in 2009 provided an ideal opportunity to focus research on the efficacy of coaching.

1 The ICF is the leading global organisation advancing the coaching profession with over 20,000 members worldwide 
There are few empirical studies which link coaching to improved results. From a managerial point of view, the issue has been that the Return on Investment (ROI) of coaching is not easily measurable (AMA, 2008). From an academic perspective, the problem is that "little guidance exists on how to evaluate this unique leadership development practice" (Ely et al., 2010, p. 585). De Haan, Duckworth, Birch and Jones (2013) have proposed that similar outcome results as in psychotherapy can be assumed. This may be sufficient for this discipline, but the business view is presumed different, as tangible results are typically required in this field.

This study focused on coaching leaders in organisations. It drew on resources from the fields of leadership, management, psychology and training \& development. The question it has aspired to answer is: "What does the application of an integrated evaluation framework tell us about the effectiveness of leadership coaching?"

Findings from the meta-analysis of research projects on the impact of coaching from Theeboom et al. (2014), Ely et al. (2010) and De Meuse et al. (2009), in combination with the work of Donald Kirkpatrick (1996) on evaluating training programs and Chen (1989) on the evaluation of program theory, support the development of an integrated evaluation framework:

\section{Integrated Evaluation Framework}

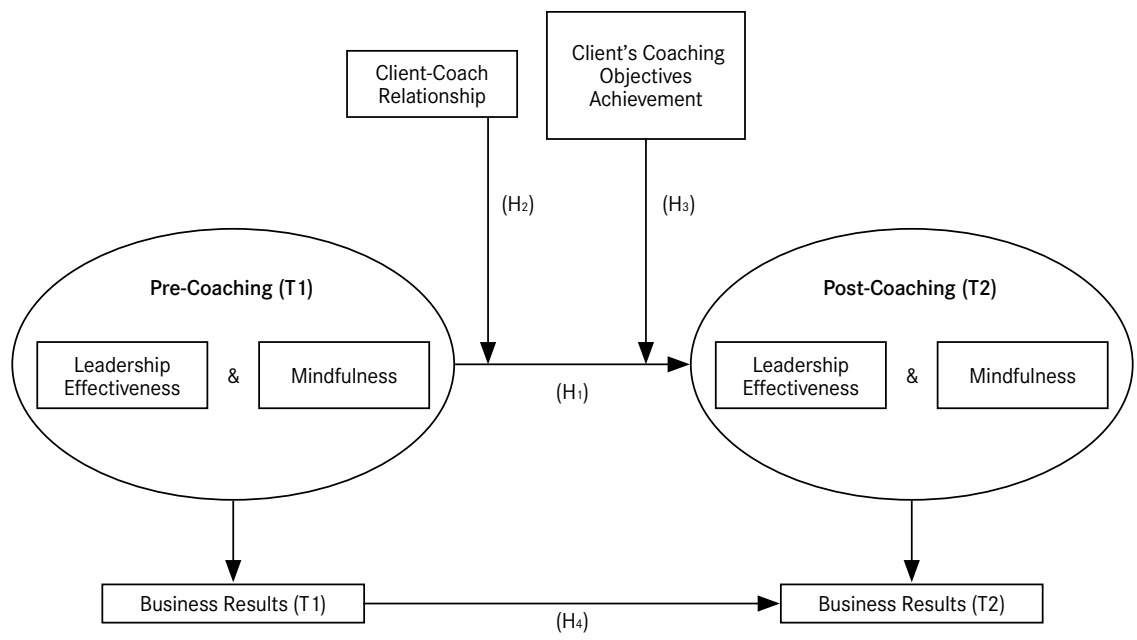

Figure 1: Integrated evaluation framework 
Leadership "involves persuading other people to set aside for a period of time their individual concerns and to pursue a common goal that is important for the responsibilities and welfare of a group", and "concerns building cohesive and goal-oriented teams; there is a causal and definitional link between leadership and team performance" (Hogan \& Curphy, 1994, p. 493). Leadership effectiveness is this link between the leader and organisational performance. Leadership coaching is "a relationship between a client and a coach that facilitates the client becoming a more effective leader" (Ely et al., 2010, p. 585).

The theory of resonant leadership of Goleman, Boyatzis and McKee (2013) is a basis for this research. This theory is closely tied to both emotional intelligence (EQ) and mindfulness, as one of the three elements supporting renewal. Both are linked to a measurement of leadership effectiveness from a sustainable, long-term point of view. Mindfulness is "being aware, awake and attending to ourselves and the way around us" (McKee \& Boyatzis, 2006, p. 34). The origins of mindfulness stem from Buddhist philosophy and are focused on the significance of consciousness. Being alert to mental contents as well as accepting and not judging are key parts of mindfulness. The assertion is that people who are mindful tend to be faster, have better ideas, are happier in life and, most crucially within the context of this research, are usually more effective. Passmore and Marianetti (2007) report that mindfulness is used in different fields, amongst those is the corporate world. They also mention various studies in which the significant benefits of mindfulness training, amongst which improvements in physiology (e. g. well-being, reduced blood pressure and cortisol levels), satisfaction (with the job), communication, creativity and productivity were reported. These research projects also showed a decrease in stress, tension and anxiety.

Self-reporting by clients and/or coaches in outcome studies has been described as an issue, because of the potential lack of the objectivity of results. Collection of multi-source data, consideration of multi-level effects and formative evaluations of the client, coach, client-coach relationship and coaching process have, therefore, been recommended. According to De Meuse et al. (2009), only three publications have focused on executive coaching engagements, provided by an external coach, based on a pre/post-coaching design and with reported statistics in the article.

The relevance of this research for the field of management is that it aims to provide a framework for managers to evaluate the effectiveness of executive/ leadership coaching through the application of an integrated evaluation framework. In terms of the academic fields of management, leadership and psychology, this research aspires to provide empirical evidence regarding the impact of leadership coaching engagements. 
The hypotheses, which consider summative, i. e. changes in outcomes (H1 and $\mathrm{H} 4)$, and formative evaluations of two key aspects of coaching ( $\mathrm{H} 2$ and $\mathrm{H} 3$ ), as depicted in Figure 1, are:

(H1) Coaching outcomes ${ }^{\star 2 *}$ have improved after leadership coaching.

(H2) The relationship between client and coach has a positive impact on coaching outcomes.

(H2.a) Client satisfaction with the coach and (H2.b) coach satisfaction with the client have a positive impact on coaching outcomes.

(H3) The achievement of the client's coaching objectives has a positive impact on coaching outcomes.

(H4) Business results are better after leadership coaching.

\section{Methods}

Pre-coaching (T1) and post-coaching (T2) data was collected between July 2011 and November 2014 for $\mathrm{N}=30$ client engagements with three female and three male professional external coaches. The clients included 11 women and 19 men: 20 worked for the same company, five for another company and the other five for different companies. Of the clients, 13 worked with the author as their coach, 15 (6, 6 and 3 each) with the three female coaches and one each with the two other male coaches. Characteristics of the coaching programme are described in Table 1.

Table 1: Descriptive statistics of the coaching programme

\begin{tabular}{|l|l|l|l|l|l|l|}
\hline & N & Range & Min & Max & Mean & $\begin{array}{l}\text { Standard } \\
\text { deviation }\end{array}$ \\
\hline $\begin{array}{l}\text { length_leadership_ } \\
\text { coaching (in months) }\end{array}$ & 30 & 3 & 8 & 11 & 8.67 & .884 \\
\hline $\begin{array}{l}\text { number_of_coaching } \\
\text { sessions }\end{array}$ & 30 & 8 & 6 & 14 & 11.83 & 2.408 \\
\hline number_of_hours & 30 & 28 & 8 & 36 & 16.59 & 4.995 \\
\hline $\begin{array}{l}\text { average_duration_coach- } \\
\text { ing session (in hours) }\end{array}$ & 30 & 1.57 & 1.00 & 2.57 & 1.4097 & .29530 \\
\hline $\begin{array}{l}\text { number_of_3ways_with_- } \\
\text { boss }\end{array}$ & 30 & 3 & 1 & 4 & 2.73 & .868 \\
\hline Valid N (listwise) & 30 & & & & & \\
\hline
\end{tabular}

$2^{*}$ Coaching outcomes as measured by leadership effectiveness and mindfulness. 
For the 30 client engagements a total of $\mathrm{N}=376$ evaluators completed $360^{\circ}$ feedback during the pre-coaching and $\mathrm{N}=310$ during the post-coaching phase. 208 of these $310(67.1 \%)$ were the same evaluators as during the pre-coaching phase.

The following instruments were used to collect the data as described in Figure 2:

- The Leadership Circle Profile ${ }^{\text {mix }}$ (TLCP), a 124-item scientifically validated multi-source instrument with the responses based on a nine-point Likert scale (Anderson Jr., 2006b), which includes a summary measure for leadership effectiveness.

- The Freiburg Mindfulness Inventory (FMI), a 14-item semantically robust and psychometrically stable, validated tool based on a four-point Likert scale (Walach, Buchheld, Buttenmüller, Kleinknecht \& Schmidt, 2006).

- The Business Performance Index (BPI), a 6-item tool developed by TLCP, collects business results data from clients and their bosses based on a seven-point Likert scale.

- An evaluation of the coaching programme both by the client - an 11-item questionnaire, and the coach - a 10-item questionnaire, and in terms of the achievement of the coaching objectives, all using a five-point Likert scale.

\section{Coaching Programme}

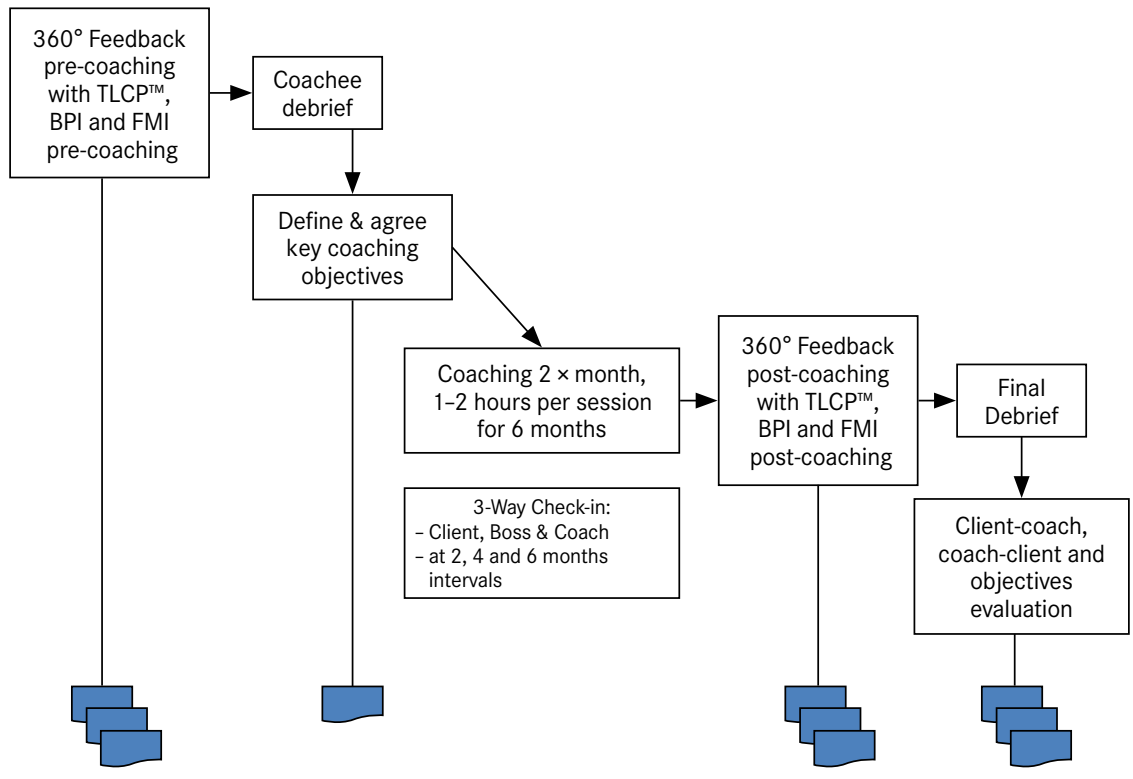

Figure 2: Coaching programme 
The variables within the research model are expressed in the following way:

- Coaching outcomes as expressed by the difference between TLCP leadership effectiveness (all evaluators) and FMI mindfulness both before and after leadership coaching.

- Business results as reported by the difference of overall performance on the Business Performance Index (BPI), as assessed by the boss, before and after leadership coaching.

- Client satisfaction, which refers to the client's level of satisfaction with the coach as derived from the client's evaluation of the coach.

- Coach satisfaction, which refers to the coach's level of satisfaction with the client as derived from the coach's evaluation of the client.

- The Client-Coach Relationship Index (CCRI) as calculated by dividing the client satisfaction score by the coach satisfaction score with an index of 1.00 indicating a well-balanced relationship; variations indicate that the client either experienced the relationship as more $(>1.00)$ or less $(<1.00)$ positive than the coach.

- The Client's Coaching Objectives Achievement (CCOI) is the average of the scores for the objectives, with a score of 1.00 indicating that the objectives have been achieved, a score of below and above 1.00 indicating that the objectives have not been achieved and exceeded respectively.

Correlations between the key variables of the integrated evaluation framework are summarised in Table 2:

Table 2: Correlation of key variables. $N=30$, except for Business Performance Index $(N=27)$; ${ }^{\star} \mathrm{p}<.05 ;{ }^{* *} \mathrm{p}<.01$. Cronbach's alphas appear along the diagonal in parentheses.

\begin{tabular}{|l|l|l|l|l|l|l|l|l|l|}
\hline Variable & M & SD & 1 & 2 & 3 & 4 & 5 & 6 & 7 \\
\hline $\begin{array}{l}\text { 1.Client-Coach } \\
\text { Relationship } \\
\text { Index }\end{array}$ & 1.08 & .13 & --- & & & & & & \\
\hline $\begin{array}{l}\text { 2. Client } \\
\text { Satisfaction }\end{array}$ & 49.93 & 3.30 & $.37^{\star}$ & $(.82)$ & & & & & \\
\hline $\begin{array}{l}\text { 3.Coach } \\
\text { Satisfaction }\end{array}$ & 46.90 & 5.07 & $-.85^{\star \star}$ & .17 & $(.86)$ & & & & \\
\hline $\begin{array}{l}\text { 4.Coaching Ob- } \\
\text { jectives Achieve- } \\
\text { ment Index }\end{array}$ & .96 & .22 & .00 & .35 & .17 & --- & & & \\
\hline $\begin{array}{l}\text { 5.Leadership Ef- } \\
\text { fectiveness_diff } \\
\text { (all evaluators) }\end{array}$ & .09 & .34 & -.12 & -.25 & .02 & -.18 & $(.95)$ & & \\
\hline
\end{tabular}




\begin{tabular}{|l|l|l|l|l|l|l|l|l|l|}
\hline Variable & $\mathbf{M}$ & SD & $\mathbf{1}$ & $\mathbf{2}$ & $\mathbf{3}$ & $\mathbf{4}$ & $\mathbf{5}$ & $\mathbf{6}$ & $\mathbf{7}$ \\
\hline $\begin{array}{l}\text { 6.Mindfulness_ } \\
\text { diff }\end{array}$ & 4.35 & 4.76 & $-.46^{\star}$ & -.09 & $.45^{\star}$ & .08 & .32 & $(.81)$ & \\
\hline $\begin{array}{l}\text { 7.Business } \\
\begin{array}{l}\text { Performance In- } \\
\text { dex_diff (boss) }\end{array}\end{array}$ & .02 & 1.04 & -.07 & .04 & .07 & -.08 & .14 & -.03 & ---- \\
\hline
\end{tabular}

The strong negative correlation between the T1-T2 difference in mindfulness and the Client-Coach Relationship Index (CCRI) indicates that if a coach is less satisfied with the client than the client is with the coach, mindfulness is impacted negatively and vice versa as corroborated by the strong positive correlation between the change in mindfulness and coach satisfaction. CCRI has a strong negative correlation with coach satisfaction, with lower levels of coach satisfaction associated with higher levels of CCRI.

Three areas were analysed to investigate possible impact on the results:

1. 20 of the 30 clients were part of a leadership development programme that included 5 days of workshops in addition to the coaching. An independent-samples t-test suggested no significant difference in the scores for those who attended the leadership programme in terms of coaching outcomes and the ones who did not.

2. Eight clients had a change in boss. Independent-samples t-tests comparing key variables, in which the boss was involved in evaluating clients who had a change in boss during the programme and for those who did not, showed that the differences were insignificant (effect sizes as measured by eta squared ranged from.00 to.04).

3. A mixed within subjects and in-between subjects analysis of variance showed no significant correlations or interactions between the number of sessions, hours and length of leadership coaching, the number of 3-way conversations between client, boss and coach, client and coach demographics with coaching outcomes, except between coach gender and the change in mindfulness.

A paired samples t-test will be performed to test $\mathrm{H} 1$ and $\mathrm{H} 4$ and the related sub-hypotheses. Given the sample size of $\mathrm{n}=30$ ANOVA (analysis of variance) and applying Bonferroni confidence interval adjustment, it will be used to test the interactions of hypotheses and sub-hypotheses for $\mathrm{H} 2$ and $\mathrm{H} 3$. Since there will be an opportunity to analyse the data, in particular the multi-source data, from multiple points of view, the results will also be analysed in terms of these different sources, i. e. self, boss, boss's boss, direct reports, peers and others, where appropriate. 


\section{Results}

The paired-samples t-tests to evaluate $\mathrm{H} 1$ showed that the change in leadership effectiveness (all evaluators) $(\mathrm{M}=.09, \mathrm{t}(29)=1.28, \mathrm{p}=.212)$, though positive, was not statistically significant. However, mindfulness had increased significantly with a mean increase in mindfulness scores of 4.35 ( $\mathrm{p}<.001)$ with a $95 \%$ confidence interval ranging from 2.57 to 6.13 . Cohen's (1988) $d=.75$, indicating a large ES. H1, is thus only partially accepted.

To enable comparisons with other studies, paired-samples t-tests for the change in leadership effectiveness, as evaluated by the client him/herself as well as his/ her boss, direct reports, peers and others, were also carried out. Only the changes in the boss's evaluation $(\mathrm{M}=0.27, \mathrm{t}(22)=2.30, \mathrm{p}<.05$ with a $95 \%$ confidence interval ranging from 0.03 to 0.52 with Cohen's $d=.46$ indicating a medium ES) and in the self-evaluations $(\mathrm{M}=.26, \mathrm{t}(29)=2.11, \mathrm{p}<.05$ with a $95 \%$ confidence interval ranging from. 01 to .51 with Cohen's $d=.38$ indicating a small ES) were statistically significant.

The mixed within subjects and in-between subjects analyses of variance ${ }^{3}$, used to review the impact of the various aspects of the relationship between client and coach on coaching outcomes (H2), first indicated there were no significant interactions between the CCRI and coaching outcomes. Secondly, there were significant interactions between client satisfaction and leadership effectiveness, Wilks Lambda $=.47, \mathrm{~F}(9,20)=2.53, \mathrm{p}<.05$, partial eta squared $=.53$ and mindfulness, Wilks Lambda $=.37, \mathrm{~F}(9,20)=3.79, \mathrm{p}<.01$, partial eta squared .63 . Thirdly, there were no significant interactions between coach satisfaction and coaching outcomes. Only H2.a has therefore been accepted.

Regarding the impact of the achievement of coaching objectives (CCOI) on coaching outcomes, the results of the mixed within subjects and in-between subjects analyses of variance showed no significant interaction between CCOI and leadership effectiveness, Wilks Lambda $=.42, \mathrm{~F}(11,18)=2.25, \mathrm{p}=.061$, partial eta squared.58, and CCOI and mindfulness, Wilks Lambda $=.56, \mathrm{~F}(11,18)=$ $1.29, \mathrm{p}=.303$, partial eta squared.44. This hypothesis has therefore been rejected.

The paired-samples t-test for the fourth hypothesis indicated a statistically insignificant increase in the Business Performance Index (as assessed by the bosses) and the hypothesis has therefore been rejected.

3 Bonferroni confidence interval adjustment has been applied to all analyses of variance. 


\section{Discussion}

This study shows that, whereas leadership effectiveness (all evaluators) does not show a statistically significantly increase after leadership coaching, mindfulness and leadership effectiveness (as evaluated by the bosses and the clients themselves) have increased significantly. The results in this study range from Cohen's $d=.75$ (change in mindfulness) to $d=.46$ (boss-assessed leadership effectiveness) and $d=.38$ (self-assessed Leadership Effectiveness). Table 3 shows these results within the context of other outcome studies that have reported Cohen's $d$.

Table 3: Comparison to other outcome studies

\begin{tabular}{|c|c|c|c|}
\hline Author & Outcome & Significance* & Issue \\
\hline $\begin{array}{l}\text { Peterson } \\
\text { (1993) }\end{array}$ & $\begin{array}{l}\text { Measures related to ob- } \\
\text { jectives of coaching }\end{array}$ & $d>1.5$ & $\begin{array}{l}\text { Self-reports \& } \\
\text { supervisory ratings } \\
\text { only }\end{array}$ \\
\hline $\begin{array}{l}\text { Smither et al. } \\
(2003)\end{array}$ & $\begin{array}{l}\text { Working with a coach as } \\
\text { opposed to not }\end{array}$ & $d=.17$ & $\begin{array}{l}\text { Direct reports and } \\
\text { superiors only }\end{array}$ \\
\hline $\begin{array}{l}\text { Evers et al. } \\
(2006)\end{array}$ & $\begin{array}{l}\text { Self-efficacy beliefs and } \\
\text { outcome expectancies }\end{array}$ & $d=.5$ & Self-reports only \\
\hline $\begin{array}{l}\text { Perkins } \\
(2009)\end{array}$ & $\begin{array}{l}\text { Leadership behaviours in } \\
\text { meetings }\end{array}$ & $d>.95$ & Coach ratings only \\
\hline $\begin{array}{l}\text { Nieminen et al. } \\
\text { (2013) }\end{array}$ & $\begin{array}{l}\text { Mission- and adaptability } \\
\text { focused leadership } \\
\text { behaviours }\end{array}$ & $d=.17$ and .27 & Self-reports only \\
\hline $\begin{array}{l}\text { Hofmans } \\
(2015)\end{array}$ & $\begin{array}{l}\text { Leadership Effectiveness } \\
\text { (LE) \& Mindfulness }\end{array}$ & $\begin{array}{l}\text { LE all evaluators: } \\
\text { insignificant. } \\
\text { Boss }(d=.46) \text {, } \\
\text { Self }(d=.38) \text {, Mind- } \\
\text { fulness }(d=.75)\end{array}$ & $\begin{array}{l}\text { Evaluator/rater } \\
\text { consistency }(67.1 \%) \\
\text { and Sample size } \\
(\mathrm{N}=30)\end{array}$ \\
\hline
\end{tabular}

${ }^{\star}$ Cohen's $d$ are classified as Small, $d=.2$, Medium, $d=.5$ or Large, $d=.8$.

There are three significant findings regarding the impact of the client-coach relationship on coaching outcomes. Firstly, while using a more objective assessment of the coaching outcome, the relationship between the client and the coach does not appear to significantly impact coaching outcomes. Even though set in a different environment (military), these results appear to be in line with the ones from Boyce, Jackson and Neal (2010). Baron and Morin (2009) and de Haan et al. (2013) found a high correlation between the client-coach relationship and the coaching outcome (self-report from the clients). This appears to point more towards the second finding in this study, i.e. client satisfaction significantly 
impacts coaching outcomes. Therefore, it is not so much the relationship but more how satisfied the client is with the coach that impacts the outcome of the coaching programme. While measured in a different way, this confirms the findings of de Haan et al. (2013), i. e. a strong positive correlation between how the client experiences the relationship and the coaching effectiveness.

Even though there is not a significant impact on the coaching outcome, the third finding is that the coach's assessment of the relationship with the client has a much stronger influence on the said relationship with the client than how the client evaluates this as illustrated by the correlations (Table 2). Boyce et al. (2010) and Baron and Morin (2009) also found a greater significance in the coach's rating of the relationship. Despite the insignificant interactions that have been found for coach satisfaction and coaching outcomes, the positive correlation between coach satisfaction and mindfulness $(\mathrm{r}=.45, \mathrm{n}=30, \mathrm{p}<.05)$ could possibly confirm a more significant impact of coach satisfaction on coaching outcomes, which is consistent with Boyce et al. (2010). What this suggests is that, contrary to what has been reported by de Haan et al. (2013), the way the coaches feel about the relationship with the client has a more significant influence on the relationship between client and coach, and possibly coaching outcomes, than previously believed. This strengthens the argument that "Coaches may not be as 'in tune' with their clients as they typically assume" (de Haan et al., 2013, p. 54).

A statistically significant result has not been obtained regarding $\mathrm{H} 3$, but the significance of the achievement of the client's coaching objectives with $p=.061$ appears to be very close to being a predictor for coaching outcome. It is important to highlight that an as-close-as-possible objective measure for the determination of the coaching outcome was used and the low rater consistency may have influenced the outcome. Correlations between 90 of the 95 objectives, as classified under the leadership dimensions of the Leadership Circle Profile, and coaching outcomes were strong $(\mathrm{p}<.01)$ for all four dimensions and leadership effectiveness $(.70$ to.88) and significant $(\mathrm{p}<.05)$ for the relating $(.37)$ and self awareness (.41) dimensions and mindfulness. Bowles, Cunningham, De La Rosa and Picano (2007), Evers, Brouwers and Tomic (2006) and Orenstein (2006) have also documented positive findings with regard to setting goals in coaching.

The ideal outcome would have been to objectively show an improvement in business results for the area that the leader, who has been coached, is responsible for, after leadership coaching (H4). Even though the results show a slight increase, as assessed by the leaders' bosses after coaching, they are not statistically significant. These findings are different to the ones found by Anderson (2006a, 2006b) in which a correlation of .612 was reported between leadership effectiveness and business performance. The sample size $(\mathrm{N}=486)$ was signif- 
icantly larger and only one measurement was used instead of the longitudinal measurements reported here.

\section{Limitations and implications for research and practice}

There are a number of limitations in this study. The first one has to do with the sample size. It is recognised that with a sample of $\mathrm{N}=30$ it is difficult to generalise the results. Since the literature on sample size appears to be inconsistent in terms of what is a required sample size, an area for future research could be to take the proposed framework and methodology to continue the collection of data until a sample size has been determined that allows for the results to be generalised. In addition, using a control group in the design could complement future research.

A second limitation has to do with the $67.1 \%$ rater consistency. Despite possible issues related to rater bias, multi-source feedback has been described as being closest to an objective measurement of progress. It would be interesting to find out what makes evaluators decide to either complete a request for such feedback or not and to understand the pre-requisite conditions under which evaluators will complete both multi-source feedback requests in a pre/ post-coaching research design.

Thirdly, given that self-reporting has been identified as a limitation in coaching effectiveness literature, it could be seen as a limitation that results for mindfulness if only self-assessed data has been obtained. The question is how someone else other than the individual concerned could correctly assess how mindful that person is. Given the significance of the changes in mindfulness and its importance for leadership effectiveness, another area for future research could be to include a measurement of mindfulness as experienced by others.

A fourth limitation relates to how events outside the realm of coaching have impacted the outcome. Whereas the impact of the changes of bosses has been analysed more deeply and found not to have impacted the results, the same cannot be said for the analysis of the impact of other, e. g. organisational, changes. Today's organisational world is characterised by constant change, and research into how these changes impact leadership coaching engagements could possibly assist organisations in determining the planning of these leadership development interventions.

Finally, a fifth limitation could be that two-thirds of the clients received coaching as part of a leadership development programme that also included other interventions. Given the reported statistically insignificant differences and 
the difficulties encountered in obtaining an acceptable sample size, the belief is that it has been a reasonable choice to include the data for all these coaching engagements in this study. Based on the results from Olivero, Bane and Kopelman (1997), in which they found training only increased productivity by $22.4 \%$, training-with-coaching increased productivity by $88 \%$ in a public agency. The results of this study further reinforce the impact of coaching as a developmental intervention. Nevertheless, another area for future research would be to only focus on coaching engagements without any other types of leadership development mixed in. This would further validate the finding of this and other empirical research that coaching, as a standalone leadership development intervention, produces positive results.

\section{References}

AMA (2008). Coaching: A Global Study of Successful Practices. New York, NY: American Management Association.

Anderson Jr., R. J. (2006a). The Leadership Circle and Organizational Performance.http://execedprograms.nd.edu/assets/190513/the_leadership_circle_and_organizational_performance.pdf [17.3.3017].

Anderson Jr., R. J. (2006b). The Leadership Circle Profile: breakthrough leadership assessment technology. Industrial \& Commercial Training, 38 (4), 175-184. doi.org/10.1108/00197850610671946.

Baron, L., Morin, L. (2009). The Coach-Coachee relationship in Executive Coaching: A Field Study. Human Resource Development Quarterly, 20 (1), 85-105.

Bowles, S., Cunningham, C. J. L., De La Rosa, G. M., Picano, J. (2007). Coaching leaders in middle and executive management: Goals, performance, buy-in. Leadership \& Organization Development Journal, 28 (5), 388-408. doi.org/10.1108/01437730710761715.

Boyce, L. A., Jackson, R. J., Neal, L. J. (2010). Building successful leadership coaching relationships: Examining impact of matching criteria in a leadership coaching program. Journal of Management Development, 29 (10), 914-931. doi.org/10.1108/02621711011084231

Chen, H. (1989). The conceptual framework of the theory-driven perspective. Evaluation and Program Planning, 12 (4), 391-396. doi.org/10.1016/0149-7189(89)90057-8.

De Meuse, K. P., Dai, G., Lee, R. J. (2009). Evaluating the effectiveness of executive coaching: Beyond ROI? Coaching: An International Journal of Theory, Research and Practice, 2 (2), 117-134. doi. org/10.1080/17521880902882413.

Ely, K., Boyce, L. A., Nelson, J. K., Zaccaro, S. J., Hernez-Broome, G., Whyman, W. (2010). Evaluating leadership coaching: A review and integrated framework. The Leadership Quarterly, 21 (4), 585-599. doi.org/10.1016/j.leaqua.2010.06.003.

Evers, W. J. G., Brouwers, A., Tomic, W. (2006). A Quasi-experimental Study on Management Coaching Effectiveness. Consulting Psychology Journal: Practice \& Research, 58 (3), 174-182. doi.org/10.1037/1065-9293.58.3.174.

Goleman, D., Boyatzis, R. E., McKee, A. (2013). Primal Leadership: Unleashing the Power of Emotional Intelligence ( $10^{\text {th }}$ ed.). Boston, MA: Harvard Business Review Press.

Haan, E. de, Duckworth, A., Birch, D., Jones, C. (2013). Executive coaching outcome research: The contribution of common factors such as relationship, personality match, and self-efficacy. Consulting Psychology Journal: Practice and Research, 65 (1), 40-57. doi.org/10.1037/a0031635. 
Hogan, R., Curphy, G. J. (1994). What we know about leadership? American Psychologist, 49 (6), 493. Kirkpatrick, D. (1996). Great ideas revisited. Training \& Development, 50 (1), 54.

McKee, A., Boyatzis, R. E. (2006). Renewing and sustaining leadership. Leader to Leader, 2006 (40), 30-36. doi.org/10.1002/ltl.175.

Olivero, G., Bane, K. D., Kopelman, R. E. (1997). Executive coaching as a transfer of training tool: Effects on productivity in a public agency. Public Personnel Management, 26 (4), 461.

Orenstein, R. L. (2006). Measuring Executive Coaching Efficacy? The Answer Was Right Here All the Time. Consulting Psychology Journal: Practice \& Research, 58 (2), 106-116. doi. org/10.1037/1065-9293.58.2.106.

Passmore, J., Marianetti, O. (2007). The role of mindfulness in coaching. The Coaching Psychologist, 3 (3), 130-136.

Theeboom, T., Beersma, B., Vianen, A. E. M. van (2014). Does coaching work? A meta-analysis on the effects of coaching on individual level outcomes in an organizational context. The Journal of Positive Psychology, 9 (1), 1-18.

Walach, H., Buchheld, N., Buttenmüller, V., Kleinknecht, N., Schmidt, S. (2006). Measuring mindfulness--the Freiburg Mindfulness Inventory (FMI). Personality and Individual Differences, 40 (8), 1543-1555. doi.org/10.1016/j.paid.2005.11.025. 


\section{Teil 2}

Forschungsrelevante Beiträge aus der Praxis 
(c) 2018, Vandenhoeck \& Ruprecht $\mathrm{GmbH} \&$ Co. KG, Göttingen https://doi.org/10.13109/9783666402975 | CC BY-NC-ND 4.0 


\section{Formatkompetenz von Coaches}

Design von maßgeschneiderten Vorgehensweisen im Coaching

Elke Berninger-Schäfer und Efriom Kineselassie

Trendstudien weisen auf die zunehmende Bedeutung der Spezialisierung von Coaches und ihre damit zusammenhängende »Formatkompetenz» hin. Diese Entwicklung wird durch die Möglichkeiten von Online-Anwendungen im Coaching maßgeblich unterstützt, was in diesem Beitrag an den beiden Beispielen »Teamcoaching online» und "Walk and Talk» dargestellt wird. Es werden Format- und Setting-Kriterien vorgestellt, die in unterschiedlicher Kombination Formatvielfalt ermöglichen. Design- und Formatkompetenz sind eine wesentliche Qualitätsanforderung an zukunftsfähiges, professionelles Coaching und bedürfen der wissenschaftlichen Erforschung.

\section{Stellenwert der Formatkompetenz in der Entwicklungsgeschichte des Coachings}

Coaching hat sich im deutschsprachigen Raum seit Mitte der 1980er Jahre entwickelt. Wie Böning und Fritschle (2005) ausführen, war der Kick-off des Coachings die Einzelbetreuung von Topmanagern durch externe Berater und wurde dann über die interne Beratung von mittleren und unteren Führungskräften Teil einer systematischen Personalentwicklung. Mitte der 1990er Jahre kam es zu einer weiteren Ausdifferenzierung von Zielgruppen und Formaten durch Gruppen-, Team- und Projektcoaching. In dieser Zeit stieg die Popularität von Coaching dermaßen an, dass Ende der 1990er Jahre von der »Phase des Populismus " gesprochen wird, insofern sich Berater und Trainerinnen jeglicher Couleur zum Coach ernannten, womit eine Ausweitung von Coaching auf alle möglichen Themen verbunden war (Böning \& Fritschle, 2005). In den 2000er Jahren finden sich zwei parallele Entwicklungsstränge. In Anlehnung an Birgmeier (2011) können sie einerseits als vertiefte Professionalisierung und andererseits als Professionalisierungskritik beschrieben werden. Während Zweiteres mit dem sogenannten Scharlatanerieproblem und einer Intransparenz des Marktes einherging und immer noch einhergeht, besteht die vertiefte Professionalisierung in zielgruppenspezifischen und methodisch differenzierten Anwendungen, 
Qualitätsdefinitionen, in der Standardisierung von Ausbildungen, Verbandsgründungen, Intensivierung der Forschung, Kongressen, Tagungen und dem Nachrücken jüngerer Coaches. Die weiteren Schwerpunkte der Coaching-Entwicklung vom Ende der 2000er Jahre bis heute sieht Birgmeier (2011) in der ethischen Fundierung im Coaching, der zunehmenden Coaching-Forschung als Diskurs zwischen verschiedenen Wissenschaftsdisziplinen und der Akademisierung von Coaching als Beginn einer Coaching-Wissenschaft. Dieser Trend spiegelt sich auch darin, dass seit 2015 erstmals eine deutschsprachige, wissenschaftliche Coaching-Zeitschrift erscheint: »Coaching - Theorie \& Praxis«.

Die sich abzeichnende weitere Entwicklung, wie sie sowohl Drath (2012) als auch Gross und Stephan (2015) beschreiben, besteht in einer weiteren Differenzierung von Coaching-Themen, -Settings und -Zielgruppen und einer damit zusammenhängenden Segmentierung des Marktes. Darauf müssten sich laut Gross und Stephan (2015) Coaches einstellen, wenn sie zukunftsfähig sein wollten. Die Entwicklung hängt insbesondere mit dem Einsatz neuer Medien und der daraus resultierenden Formatvielfalt zusammen. Formatkompetenz ist somit ein recht neues Kompetenzfeld im professionellen Coaching.

Im Folgenden wird anhand der beiden Fallbeispiele »Teamcoaching online» und »Walk and Talk« die Kombination verschiedener Formate dargestellt, anschließend werden Format- und Setting-Faktoren diskutiert. Diese Diskussion stellt bestenfalls einen Anfang dar, der die Notwendigkeit wissenschaftlicher Forschung im weiten Feld der Formatkompetenz im Coaching aufzeigt.

\section{Formatbeispiele}

Die beiden Beispiele "Teamcoaching online« und »Walk and Talk» stellen Coaching-Abläufe dar, die nach dem systemisch-lösungsorientierten Coaching-Prozess der »Karlsruher Schule« durchgeführt wurden (Berninger-Schäfer, 2011; Berninger-Schäfer \& Wolf, 2011; Berninger-Schäfer, 2015). Es handelte sich jeweils also um einen strukturierten Coaching-Prozess, der in definierten Phasen verläuft. Die Phasen basieren auf klientenzentrierten, hypnosystemischen und neurowissenschaftlichen Wirkfaktoren im Coaching (Grant, 2013; Grant, 2014; Grawe, Donati \& Bernauer, 1994; Grawe, 2000; Greif, 2008; Greif, 2015; Greif, Schmidt \& Thamm, 2012; Künzli, 2009; Künzli \& Seiger, 2011; Rogers, 1972). Wesentlich dabei ist die Entwicklung von Zielen aus einer positiven Befindlichkeit und nicht aus einem Problemzustand heraus, da die Wahrscheinlichkeit gelingender Umsetzung steigt, wenn Ziele emotional positiv verankert sind (Gigerenzer, 2007; Gollwitzer, Kuhl \& Heckhausen, 1996; Hüther, 2010; Schmidt, 2004; Storch, 2005; Storch, Cantieni, Hüther \& Tschacher, 2011). 
Die genannten Phasen sind in der CAI ${ }^{\circledR}$ World ${ }^{1}$ abgebildet. Wenn sie dort angeklickt werden, stehen phasenbezogene Fragesets (zur Auswahl oder Inspiration) und weitere Online-Tools zur Verfügung. Dazu gehören Aufstellungstools in 2-D- und 3-D-Welten, diverse Visualisierungsmöglichkeiten, Bildmaterialien usw. Die CAI ${ }^{\circledR}$ World kann für Online-Coaching genutzt oder als »Tool-Koffer « im Face-to-Face-Coaching eingesetzt werden. Es handelt sich dabei um jeweils andere Formate, wie im Folgenden aus den Beispielen »Teamcoaching online « und »Walk and Talk« deutlich wird. Die Screenshot-Abbildungen geben einen Eindruck von den beschriebenen Online-Tools.

\subsection{Teamcoaching online}

Teamcoaching stellt Coaches vor große Herausforderungen, da es ihnen gelingen muss, ein gemeinsames Anliegen der Teammitglieder herauszuarbeiten genauso wie gemeinsame Ziele und Umsetzungsmaßnahmen, mit denen sich alle Beteiligten identifizieren können. Meier (2004) und Topan (2011) beschreiben den solution circle als Vorgehensweise im Coaching, der mit dem Coaching-Prozess der »Karlsruher Schule« gut umgesetzt werden kann. Das folgende Beispiel wurde nach diesem Prozess durchgeführt; es wird hier in Auszügen wiedergegeben.

\section{Beispiel}

Das Team IG (interne Gesundheitsberatung) eines Unternehmens bittet um ein Teamcoaching und erhält zehn Stunden bewilligt. Anlass ist eine hohe Auslastung der fünf Teammitglieder, die seit einer innerbetrieblichen Umstrukturierung stetig zunimmt, sodass keine Zeit mehr bleibt für wichtige Absprachen untereinander, für strategische Überlegungen zur Positionierung des eigenen Dienstes und für Supervision. Den einzelnen Teammitgliedern sind für ihre Tätigkeit verschiedene Bundesländer zugeordnet, in denen der Betrieb Niederlassungen hat und für die sie jeweils zuständig sind. Aufgrund der regionalen Verteilung der Teammitglieder wurde das Teamcoaching online durchgeführt. Aufgabe des Teams ist die Unterstützung von Maßnahmen des betrieblichen Gesundheitsmanagements, wozu zum Beispiel die individuelle Gesundheitsberatung für die Mitarbeitenden vor Ort gehört, aber auch die Moderation von Gesundheitszirkeln und die Durchführung von Gesundheitsseminaren. Die problematische Situation ist dadurch entstanden, dass die jeweiligen Aufgaben nach der Umstrukturierung verschiedenen Bereichen zugeordnet wurden. Dies wurde

$1 \quad \mathrm{CAI}^{\circledR}$ World ist eine multimediale Plattform, die für Online-Coaching entwickelt wurde. Sie kann über Lizenzmodelle von Coaches genutzt werden (https://cai-world.com). 
transparent, als in der Situationsbeschreibung mit dem Online-Aufstellungstool gearbeitet wurde. Abbildung 1 zeigt eine Symbolisierung der verschiedenen Organisationseinheiten, die mit ihren Erwartungshaltungen und mangelnder gegenseitiger Abstimmung zur Problemsituation beitragen.

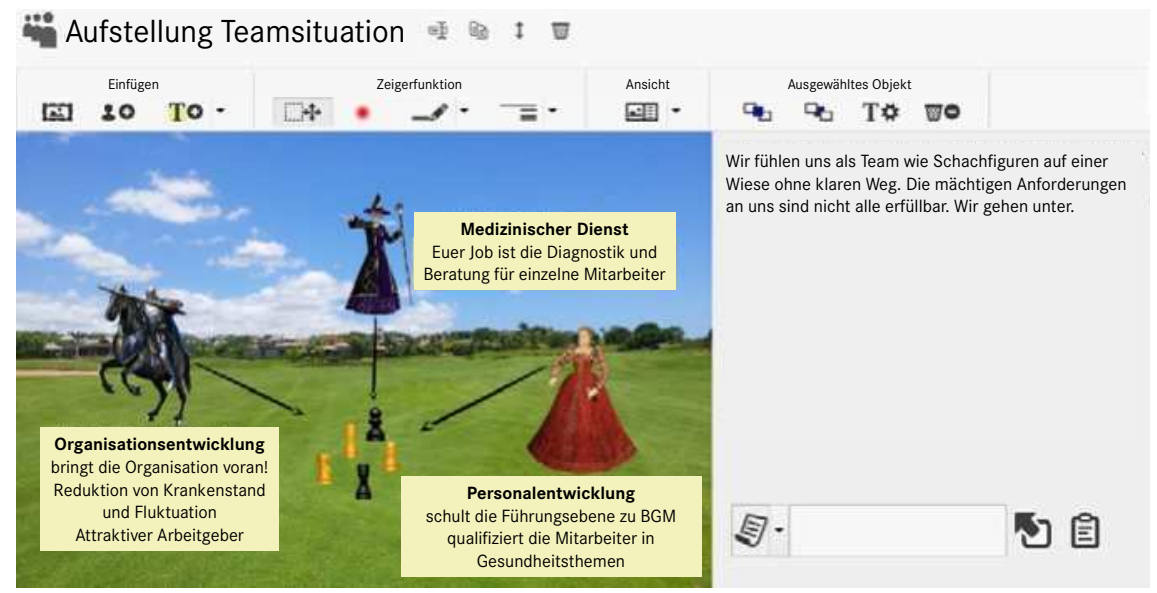

Abbildung 1: Aufstellung der Ist-Situation im Teamproblem

Nachdem die Teammitglieder sich auf ein neues Hintergrundbild mit einem vorgegebenen Weg geeinigt hatten, positionierten sie sich selbst auf dem Weg und änderten die Größenverhältnisse und Anordnungen. Sobald das Zielbild erstellt war, wurden Ziele und Maßnahmen abgeleitet. Sie sind in Abbildung 2 aufgeführt und mit unterschiedlichen Farben gekennzeichnet.

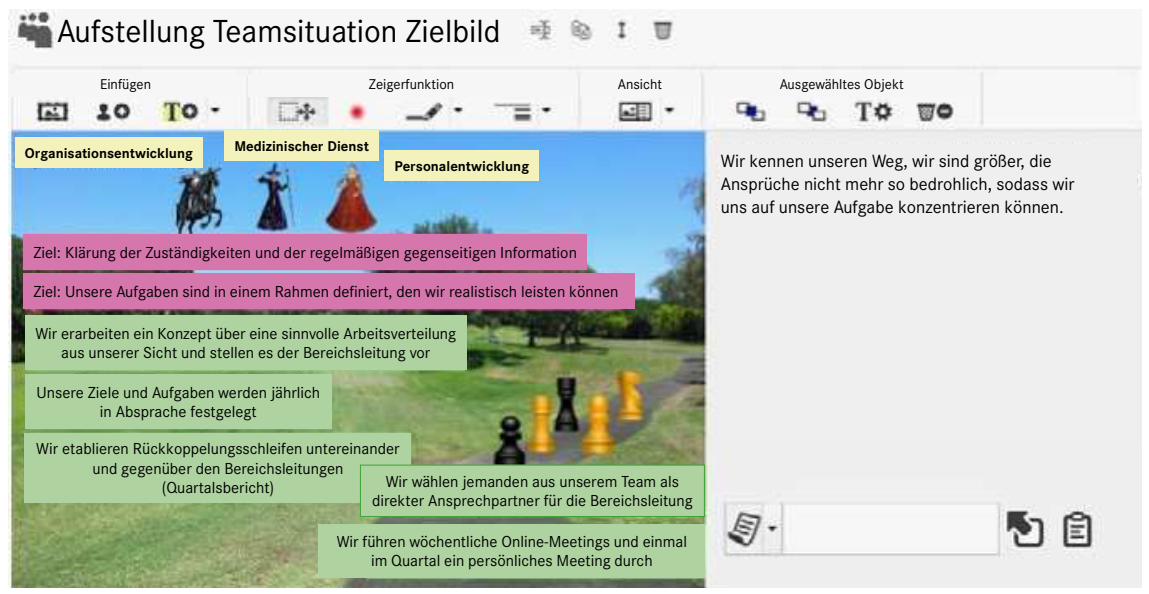

Abbildung 2: Aufstellung der Zielsituation im Team 


\subsection{Walk and Talk}

Manche Coaches bieten ihren Klientinnen und Klienten an, dass das Coaching-Gespräch im Freien stattfinden kann, während eines gemeinsamen Spazierganges, beim Walken oder Joggen. Dies wird sowohl im Gesundheitsals auch im Businesscoaching praktiziert.

\section{Beispiel}

Die Klientin im vorliegenden Beispiel ist Abteilungsleiterin in einer Non-Profit-Organisation und möchte sich mit ihrem Arbeitsstil auseinandersetzen. Sie neige dazu, sich zu überlasten, das sei ihr auch in ihren früheren Arbeitssituationen so ergangen. Es habe allerdings auch Zeiten gegeben, in denen sie sich sportlich betätigt und Zeit für ihr Hobby gehabt habe, das Malen. Beides habe ihr gutgetan, und sie wünsche sich, dass es wieder in ihrer Tagesgestaltung Platz finde. Mit dem Aufstellen von Wochenplänen komme sie aber nicht weiter. Dass das Coaching während einer Walking-Runde stattfinden kann, begeistert sie.

Beim gemeinsamen Walking von Coach und Klientin wird an einer vom Coach ausgesuchten Stelle eine Pause eingelegt. Beide nehmen auf einer Bank Platz. Auf dem mitgebrachten Tablet wird mit »inneren Anteilen « gearbeitet (Schultz von Thun, 2013). Zunächst wird das Ist-Bild erstellt, so, wie die Klientin ihre innere Situation in der Phase der Arbeitsüberlastung empfindet (vgl. Abbildung 3).

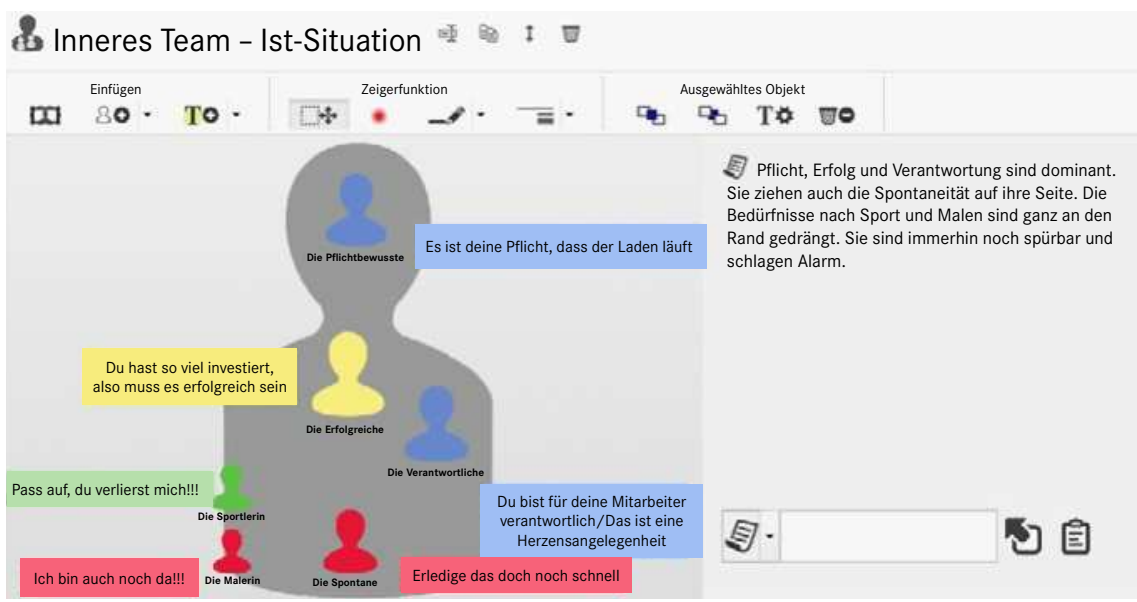

Abbildung 3: Inneres Team - Problemsituation Arbeitsüberlastung 
Wenn einmal die Ist-Situation erarbeitet und reflektiert ist, setzen Coach und Klientin ihr Walking fort. Damit wird eine Dissoziation von der Problemsituation erleichtert. Der Coach nutzt diese Etappe gezielt zur Aktivierung von Ressourcen, was durch das Natur- und Bewegungserlebnis leicht möglich ist. Bei einem nächsten Zwischenhalt arbeiten Coach und Klientin erneut auf dem Tablet am gewünschten Zielbild (vgl. Abbildung 4).

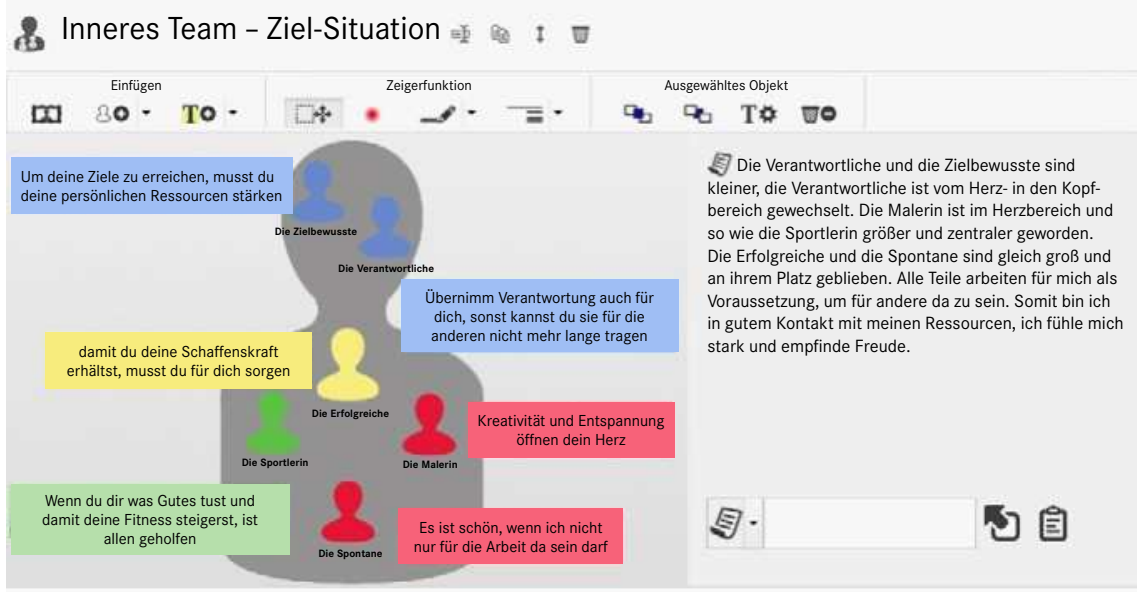

Abbildung 4: Zielbild: Integration von Ressourcen und geänderte Lebensbalance

Nach dieser assoziativ-reflexiven Pause wird die Walking-Runde fortgesetzt. Zum Schluss werten beide die gemachte Erfahrung und die Bedeutung der Bewegung für den Coaching-Prozess aus. Das innere Team ist im gemeinsamen virtuellen Coaching-Raum gespeichert und kann weiterbearbeitet werden, um den Transfer zu sichern.

Mit den beiden Beispielen "Teamcoaching online « und "Walk and Talk" sollten verschiedene Formatkriterien in jeweils unterschiedlichen Kombinationen illustriert werden. Die jeweils spezifische Kombination macht das Format aus.

Dass es sich um einen neuen Professionalisierungsbereich für Coaches handelt, ist an der relativ geringen Anzahl von Veröffentlichungen zum Thema zu erkennen (Dietz \& Müller, 2012; Geißler \& Metz, 2012). Um diese Lücke zu füllen, sollen nun abschließend die Begriffe »Format« und »Formatkompetenz« diskutiert werden. 


\section{Formatkompetenz}

Der Kompetenzbegriff bezieht sich nach Six und Gimmler (2007) auf Wissensbestände, Fähigkeiten und Fertigkeiten. Kompetenzen befähigen motivierte Individuen, Verhaltensweisen selbstbestimmt, kreativ und funktional zu realisieren. Damit ist noch keine Aussage über die verschiedenen Stufen des Kompetenzerwerbs getroffen. Insbesondere das Kennzeichen der Kreativität verlangt Virtuosität und Flexibilität, die wiederum Vielfalt voraussetzen. Damit aus einer Vielfalt von Möglichkeiten die passgenauen virtuos ausgewählt werden können, müssen die Möglichkeiten bekannt und eingeübt sein. In Bezug auf Formatkompetenz bedeutet dies in einem ersten Schritt, unterschiedliche Formate anhand ihrer Unterscheidungskriterien differenzieren zu können. Bevor solche Kriterien beschrieben werden können, ist zunächst der Gegenstand zu beschreiben, auf den sie sich beziehen, denn insbesondere beim Formatbegriff gibt es häufig Überschneidungen mit dem Setting-Begriff.

Der Begriff des »Formats « wird unter anderem als »(genormtes) Größenverhältnis eines Gegenstandes« oder als »stark ausgeprägtes Persönlichkeitsbild« (z. B. »eine Person von Format «) beschrieben (Duden online, 2016). »Setting« ist laut Duden die Gesamtheit von Merkmalen der Umgebung, in deren Rahmen etwas stattfindet beziehungsweise erlebt wird (ebd.). Format bezieht sich somit auf die Gestalt, Setting auf Umgebungsvariablen.

Die folgenden Faktoren haben Einfluss auf ein Coaching, sind allerdings im oben beschriebenen Verständnis weniger den Format- als den Setting-Kriterien zuzuordnen:

- Anbindung bzw. Beschäftigungsverhältnis der Coaches (interne Coaches, externe Coaches, Mitglied eines internen oder externen Coachpools);

- Beauftragungsform (Zwei-Personen- oder Mehrpersonen-Vertrag);

- Kontaktaufnahme (längerfristige Verabredung oder Coach-on-demand);

- Anonymität (als Möglichkeit im Online-Coaching);

- Auswahl der Coaches (durch die Klientin oder den Klienten selbst oder Vorgabe bei Mehrpersonen-Verträgen, qualitätsgesichert oder nicht);

- Themenbezug (vorhanden oder offen);

- Kontextkategorien (Profit- und Non-Profit-Bereich, Branchenbezug oder persönliches Thema).

In Abbildung 5 sind diese Setting-Faktoren in der Übersicht dargestellt. Der Vorteil der Darstellungsform liegt in der Möglichkeit zu flexiblen Kombinationen der unterschiedlichen »Kärtchen«, die entsprechend den einzelnen 
Coaching-Maßnahmen auf das geometrische Objekt gezogen werden können und somit jeweils einen anderen Coaching-Rahmen bilden.

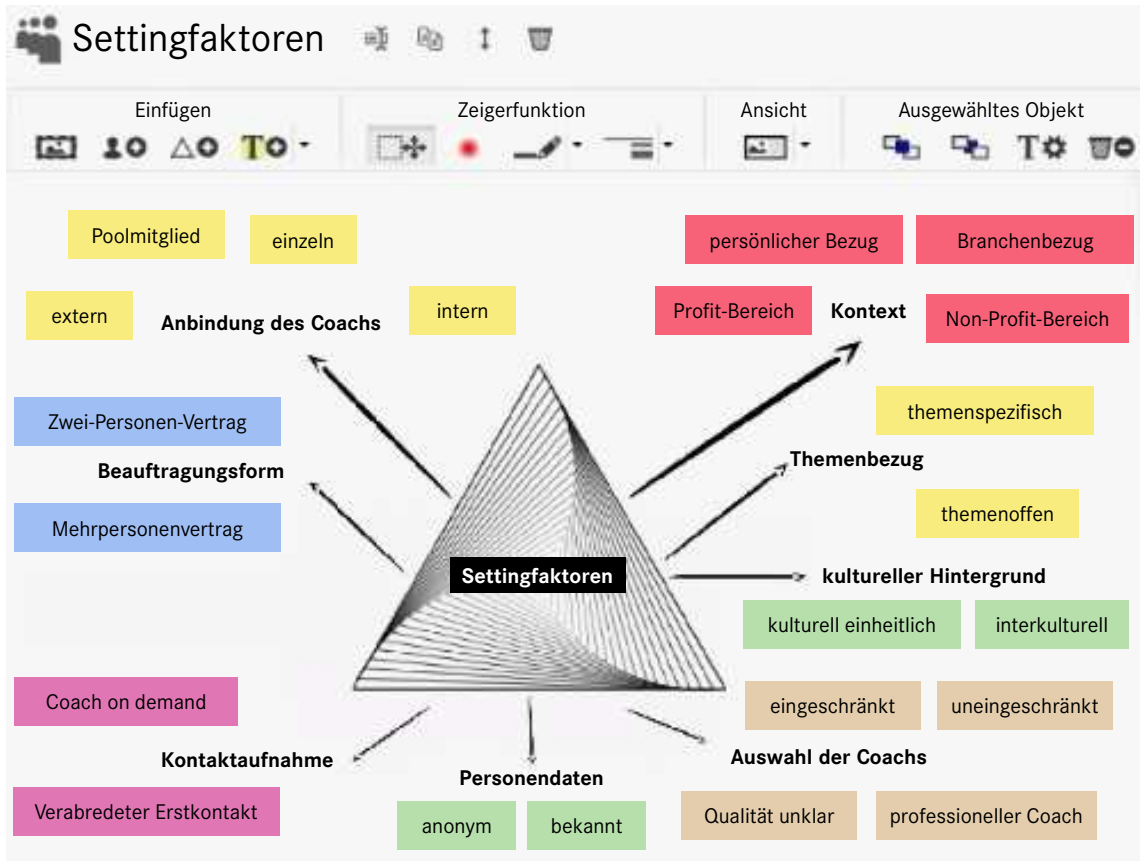

Abbildung 5: Übersicht über Setting-Faktoren im Coaching

\section{Formatkategorien}

Unterschiedliche Formate ergeben sich aus der Zusammenstellung verschiedener Formatkategorien. Innerhalb der Formatkategorien kann es wiederum weitere Unterscheidungskriterien geben, die einzeln oder in Kombination zur Unterscheidung von Formaten beitragen. Im Unterschied zu den Setting-Faktoren haben die Formatkategorien Einfluss auf die Art der Durchführung von Coaching (Form, Gestalt).

Die folgende Aufzählung erhebt keinen Anspruch auf Vollständigkeit. Sie hat eher Brainstorming-Charakter und soll einen Ausgangspunkt für weitere Überlegungen und Forschungen darstellen.

- Einbettung: Coaching als eigenständige Maßnahme oder als Programmbestandteil;

- Häufigkeit (Einzelmaßnahme oder sequenzielle Maßnahme); 
- Dauer (zeitlich begrenzt oder unbegrenzt);

- Länge der Coaching-Einheit (lang oder kurz);

- Synchronizität (zeitgleich oder zeitversetzt);

- Strukturierung des Coaching-Prozesses (offen oder strukturiert);

- Methodeneinsatz im Coaching (kommunikatives Basisverhalten mit und ohne Tool-Unterstützung);

- Beteiligte (Rolle, z. B. Coach, Führungskraft, Kollege und Anzahl, z. B. Tandem-, Gruppen-, Teamcoaching, Selbstcoaching);

- Ort des Coachings (Räumlichkeiten des Coaches, neutrale Räume, on the job, im Freien, online);

- Frequenz (niedrigfrequent oder hochfrequent);

- Medieneinsatz (face-to-face, Audio, Video, schriftlich, interaktive 2-DRäume, interaktive 3-D-Räume).

Diese Formatkategorien können wie in Abbildung 6 dargestellt aufgelistet werden:

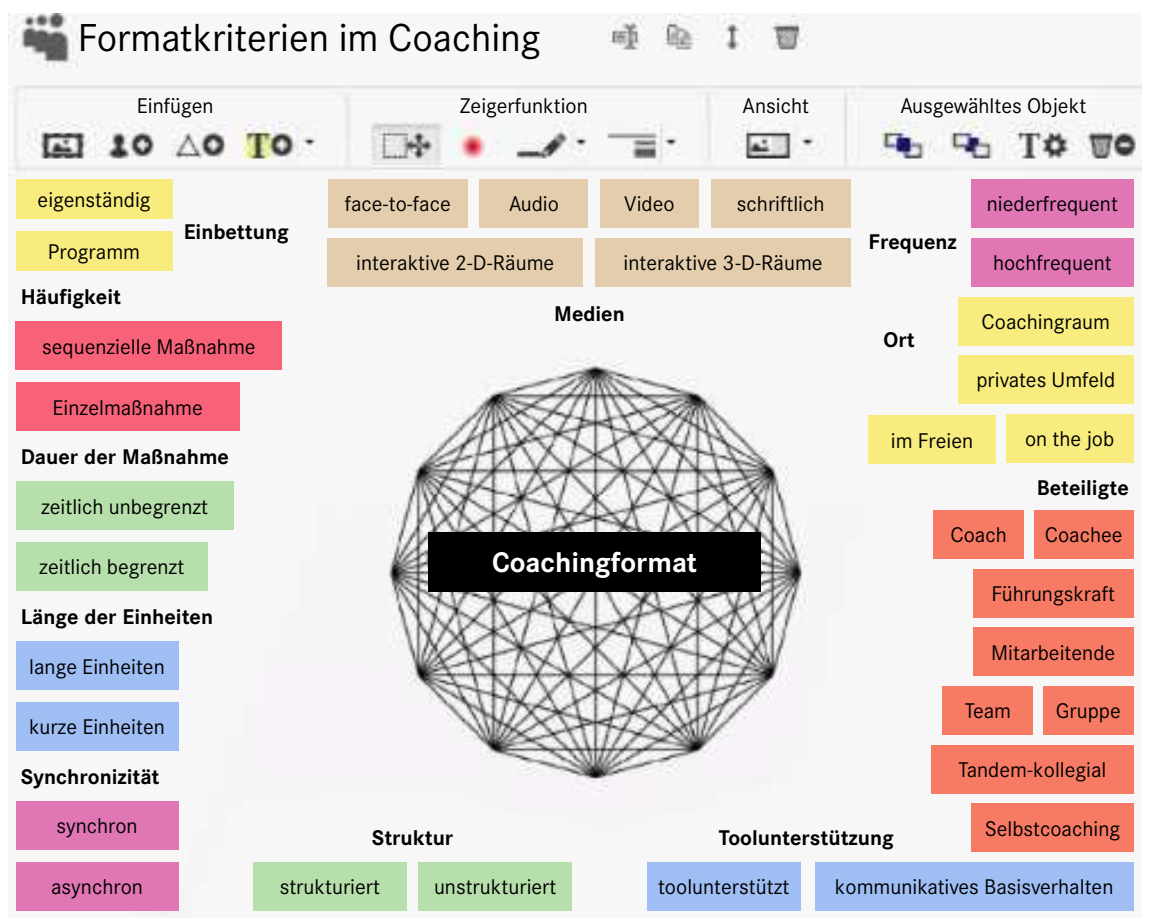

Abbildung 6: Übersicht über Formatkriterien im Coaching 
Entsprechend dieser Übersicht lässt sich das oben beschriebene Fallbeispiel "Teamcoaching online« folgendermaßen darstellen (vgl. Abbildung 7):

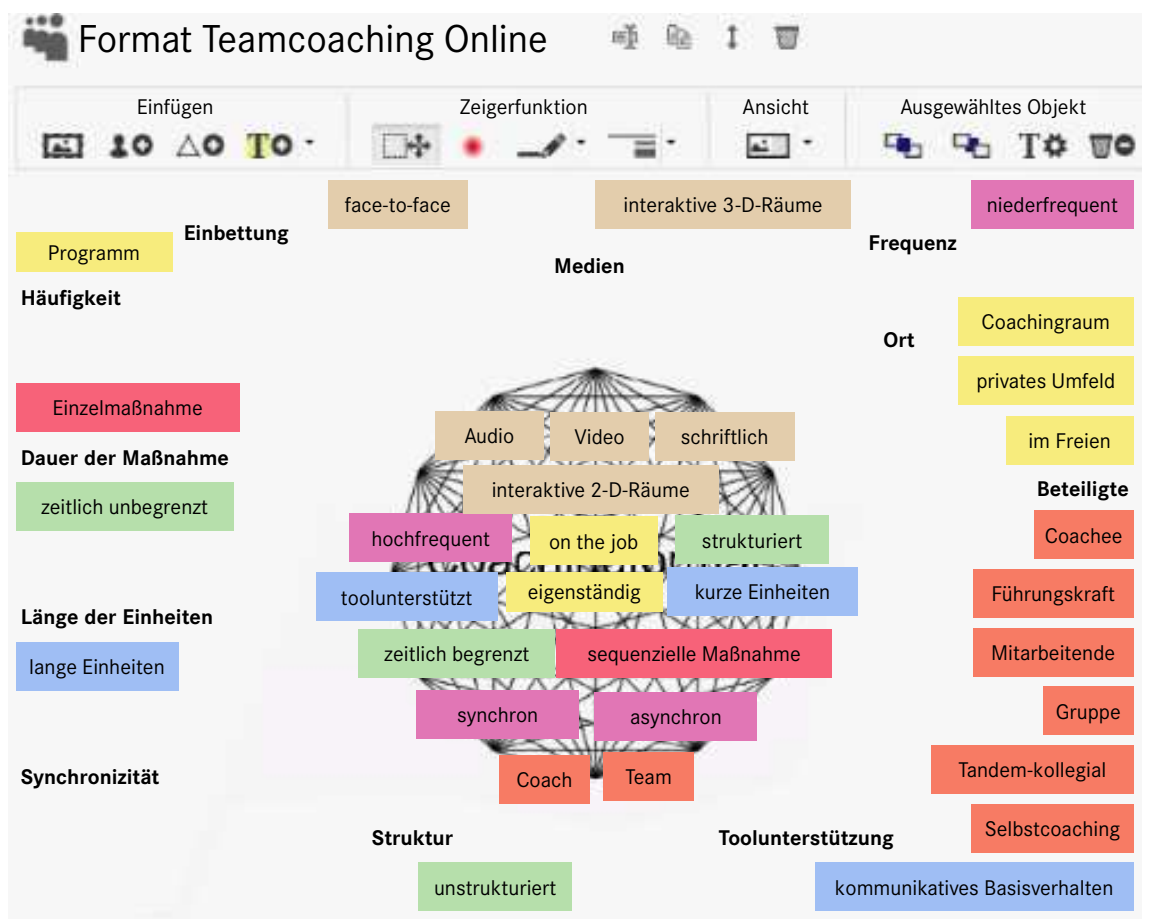

Abbildung 7: Format »Teamcoaching online«

Aus den beiden Beispielen wird die Bedeutung von Online-Vorgehensweisen für die Formatvielfalt im Coaching deutlich. Sie ermöglichen zum Beispiel hochfrequente Einheiten von kurzer Dauer, wie sie im Face-to-Face-Coaching nicht denkbar wären. Sie können auch on the job durchgeführt werden und empfehlen sich insbesondere zur Transfersicherung, das heißt in der Begleitung bei der Umsetzung von Zielen, die sich eine Klientin oder ein Klient vorgenommen hat. Studienergebnisse hierzu liegen zum Beispiel von Greif und Benning-Rohnke (2015) zu Telefon-Shadowing (TS) oder von Geißler (2011) zu dem von ihm entwickelten »Virtuellen Transfercoaching " (VTC) vor.

Die Möglichkeiten des zeitversetzten Arbeitens erweitern und verändern die Vorgehensweisen im Coaching. Sie erfordern allerdings auch besondere Kompetenzen von Coaches, zum Beispiel zum professionellen Lesen und Schreiben, zum Gestalten von Online-Beziehungen und zum Einsatz von Online-Tools, 
Das Fallbeispiel »Walk and Talk« wurde in folgendem Format verwirklicht (vgl. Abbildung 8):

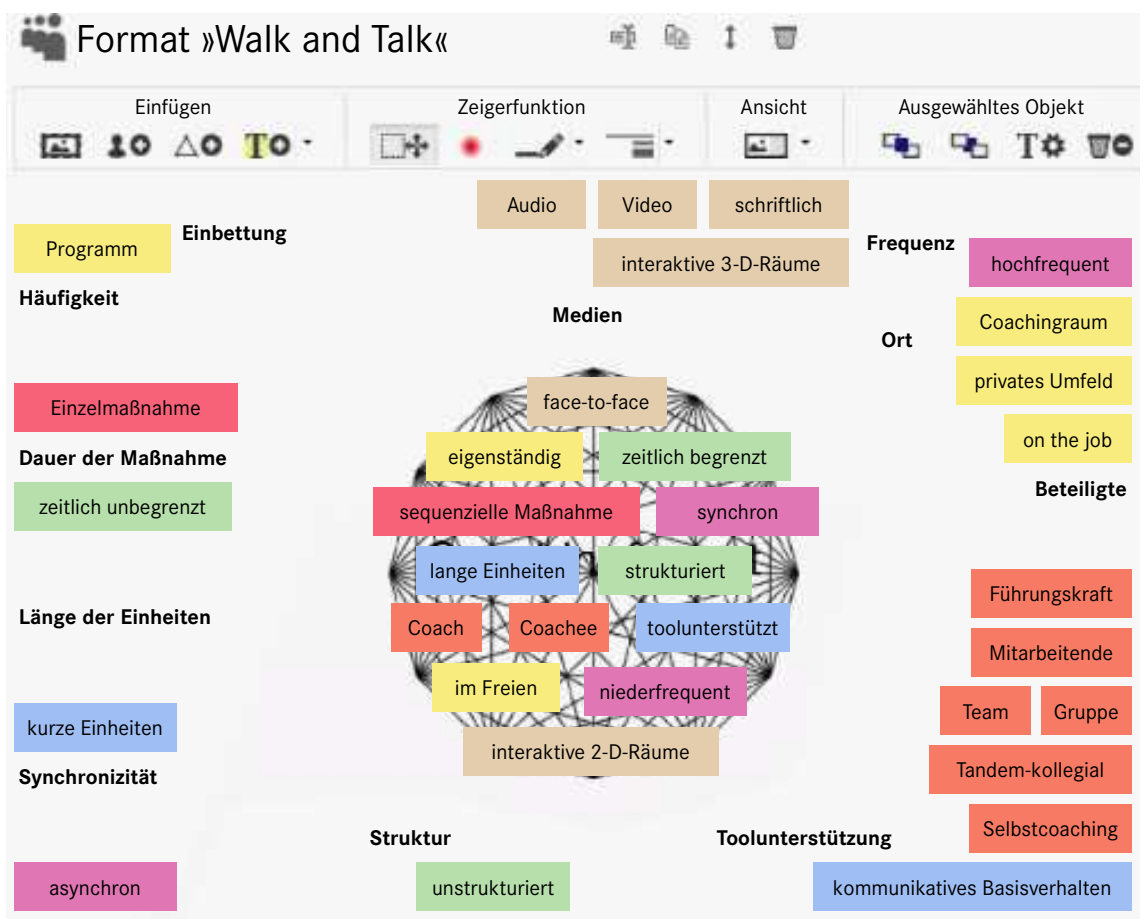

Abbildung 8: Format »Walk and Talk»

welche die Klientinnen und Klienten ermächtigen, bestimmte Schritte im Coaching-Prozess eigenständig zu gehen. Die Coaches unterstützen durch methodische Anweisungen, Feedback und Ressourcenaktivierung.

Die dargestellten Systematisierungen haben Entwurfscharakter und müssen in künftiger Forschung überprüft und gegebenenfalls modifiziert werden. Dabei ist zu beachten, dass die Flexibilität der möglichen Zusammenstellungen erhalten bleibt. Somit können Coaches maßgeschneiderte Coaching-Maßnahmen für die Anliegen der Klienten und Klientinnen zusammenstellen. 


\section{Fazit}

Formatkompetenz ist ein Qualitätsmerkmal professionellen Coachings und trägt zur Zukunftssicherung von Coaching bei. Mit den Möglichkeiten des Online-Coachings kommt ihr ein großes Entwicklungspotenzial zu. Mit ihr geht Designkompetenz Hand in Hand. Beides bedarf der Professionalisierung.

\section{Literatur}

Berninger-Schäfer, E. (2011). Orientierung im Coaching. Stuttgart: Boorberg.

Berninger-Schäfer, E. (2015). Systemisch-lösungsorientierte Bewertungskriterien der Karlsruher Schule. In H. Geißler, R. Wegener (Hrsg.), Bewertung von Coachingprozessen (S. 101-118). Wiesbaden: Springer.

Berninger-Schäfer, E., Wolf, C. (2011): Qualitätssicherung im Coaching. Was sich die Praxis von der Forschung wünscht. In E. M. Graf, Y. Aksu, I. Pick, S. Rettinger (Hrsg.), Beratung, Coaching, Supervision. Multidisziplinäre Perspektiven vernetzt (S. 85-98).Wiesbaden: VS Verlag für Sozialwissenschaften.

Birgmeier, B. (Hrsg.). (2011). Coachingwissen. Wiesbaden: VS Verlag für Sozialwissenschaften.

Böning, U., Fritschle, B. (2005). Coaching fürs Business. Was Coaches, Personaler und Manager über Coaching wissen müssen. Bonn: managerSeminare.

Dietz, T., Müller, G. (2012). Coaching-Leistungen. In DBVC (Hrsg.), Leitlinien und Empfehlungen für die Entwicklung von Coaching als Profession. Kompendium mit den Professionsstandards des DBVC (4. Aufl., S. 29-33). Osnabrück: DBVC Geschäftsstelle.

Drath, K. (2012). Coaching und seine Wurzeln. Erfolgreiche Interventionen und ihre Ursprünge. Freiburg i. Br.: Haufe.

Duden online (2016). www.duden.de [16.3.2017].

Geißler, H. (2011). Coaching meets Training - zur Lösung des Transferproblems durch »Virtuelles Transfercoaching (VTC)«. In R. Wegener, M. Loebbert, A. Fritze (Hrsg.), Coaching entwickeln. Forschung und Praxis im Dialog (S. 124-135). Wiesbaden: Springer VS.

Geißler, H., Metz, M. (Hrsg.) (2012). E-Coaching und Online-Beratung. Formate, Konzepte, Diskussionen. Wiesbaden: Springer VS.

Gigerenzer, G. (2007). Bauchentscheidungen. Die Intelligenz des Unbewussten und die Macht der Intuition. München: Bertelsmann.

Gollwitzer, P. M., Kuhl, J., Heckhausen, H. (1996). Das Rubikonmodell der Handlungsphasen. In J. Kuhl, H. Heckhausen (Hrsg.), Enzyklopädie der Psychologie. Bd, 4: Motivation, Volition und Handlung (S. 531-582). Göttingen: Hogrefe.

Grant, A. M. (2014). Autonomy support, relationship satisfaction and goal focus in the coachcoachee relationship: which best predicts coaching success? Coaching: An International Journal of Theory, Research and Practice, 7 (1), 18-38.

Grant, A. M. (2013). The efficacy of coaching. In J. Passmore, D. B. Peterson, T. Freire (Eds.), The Wiley-Blackwell Handbook of Coaching \& Mentoring Psychology (pp. 15-39). New York: Wiley Blackwell.

Grawe, K. (2000). Psychologische Therapie. Göttingen: Hogrefe.

Grawe, K., Donati, R., Bernauer, F. (1994). Psychotherapie im Wandel. Von der Konfession zur Profession (3. Aufl.). Göttingen: Hogrefe. 
Greif, S. (2015). Allgemeine Wirkfaktoren im Coachingprozess. Verhaltensbeobachtungen mit einem Ratingverfahren. In H. Geißler, R. Wegener (Hrsg.), Bewertung von Coachingprozes$\operatorname{sen}$ (S. 51-80). Wiesbaden: Springer.

Greif, S., Benning-Rohnke, E. (2015). Konsequente Umsetzung von Zielen durch Coaching Praktisch nützliche Erkenntnisse aus der Grundlagenforschung und ihre Anwendung. Coaching Theorie \& Praxis, Springer E-Journal, 1 (1), 25-35. doi:10.1365/s40896-015-0003-8.

Greif, S., Schmidt, F., Thamm, A. (2012). Warum und wodurch Coaching wirkt. Ein Überblick zum Stand der Theorieentwicklung und Forschung über Wirkfaktoren. Organisationsberatung, Supervision, Coaching, 19 (2), 275-390.

Greif, S. (2008). Coaching und ergebnisorientierte Selbstreflexion. Göttingen: Hogrefe.

Gross, P. P., Stephan, M. (2015). Der Coaching-Markt. Coaching -Theorie \& Praxis, 1 (1), 15-24. doi:10.1365/s40896-015-0002-9.

Hüther, G. (2010). Wie Embodiment neurobiologisch erklärt werden kann. In M. Storch, B. Cantieni, G. Hüther, W. Tschacher (Hrsg.), Embodiment. Die Wechselwirkung von Körper und Psyche verstehen und nutzen (S. 75-97). Bern: Huber.

Künzli, H. (2009). Wirksamkeitsforschung im Führungskräftecoaching. Organisationsberatung, Supervision, Coaching, 16 (1), 4-18.

Künzli, H., Seiger, C. (2011). Evidence-based Coaching und Wirksamkeit. Studientext zum Masterstudiengang Business Coaching und Change Management. Hamburg: Euro-FH.

Meier, D. (2004). Solution Circle für Teams. In C. Rauen (Hrsg.), Coaching-Tools (S. 300-305). Bonn: managerSeminare.

Rogers, C. R. (1972). Die nicht-direktive Beratung. München: Kindler.

Schmidt, G. (2004). Liebesaffären zwischen Problem und Lösung. Hypnosystemisches Arbeiten in schwierigen Kontexten. Heidelberg: Carl-Auer.

Schultz von Thun, F. (2013). Miteinander reden 3. Das innere Team und situationsgerechte Kommunikation. Kommunikation, Person, Situation. Reinbek b. Hamburg: Rowohlt.

Six, U., Gleich, U., Gimmler, R. (2007). Kommunikationspsychologie. In U. Six, U. Gleich, R. Gimmler (Hrsg.), Kommunikationspsychologie - Medienpsychologie. Weinheim: Beltz.

Storch, M. (2005). Das Geheimnis kluger Entscheidungen. Von somatischen Markern, Bauchgefühl und Überzeugungskraft. München: Goldmann.

Storch, M., Cantieni, B., Hüther, G., Tschacher, W. (2011). Embodiment. Die Wechselwirkung von Körper und Psyche verstehen und nutzen. Bern: Huber.

Topan, A. (2011). Teamcoaching. Stuttgart: Boorberg. 


\section{"Blended Business Coaching" mit dem Laufbahnkoffer HR}

Neue Formen des Coachings in der beruflichen Weiterbildung

Wolfgang Eberling, Gery Bruederlin und Marion Alt

Coaching hält zunehmend Einzug in berufliche Weiterbildungen. Im folgenden Beitrag wird ein modernes Blended-Coaching-Konzept vorgestellt, das an der Fachhochschule Nordwestschweiz in einem Pilotprojekt entwickelt wurde und in dem als Tool der "Laufbahnkoffer HR» (LKHR) zur Anwendung kommt. Der LKHR unterstützt bei der Entscheidung, beim Finden und Verfolgen beruflicher Zielsetzungen in Situationen der Spezialisierung oder Neuorientierung und erhöht signifikant die Selbststeuerungsmöglichkeiten von Weiterbildungsteilnehmenden. Der LKHR entspricht neuesten wissenschaftlichen Trends, die seit Längerem im therapeutischen Bereich mit computergestützten Systemen wie dem Synergetic Navigation System SNS als Erfolgsmodell erforscht werden.

Veränderungen aufgrund von Internationalisierung und Globalisierung, hohe Vernetzungsdichte und die zunehmenden Komplexität, rasante technologische Entwicklungen wie die Digitalisierung fast aller relevanten Lebensbereiche führen zwangsläufig zu immer häufigeren und schnelleren Wechseln in unseren beruflichen Handlungsfeldern. Dies geht mit ständig sich wandelnden Anforderungen an unser Wissen und unsere Kompetenzen einher und spiegelt sich auch in einer ungeheuren Flut von Weiterbildungsangeboten, die auf eine steigende Schar von Nachfragenden zu treffen scheint. Daraus ergeben sich neue Anforderungen und Möglichkeiten für Weiterbildungsanbieter. Gleichzeitig müssen veränderte Nutzergewohnheiten berücksichtigt werden, weshalb Blended Coaching mit dem Laufbahnkoffer HR gleichermaßen neue Lernformen wie auch die Professionalisierung von Coaching in solchen Transformationsphasen unterstützt. 


\section{Weiterbildung als kritischer Übergang in der beruflichen Laufbahn}

Der Entscheid für eine Weiterbildung, ihr Besuch selbst und der Transfer des Gelernten in die berufliche Praxis stellen aus Sicht von Weiterbildungsteilnehmenden häufig kritische Veränderungen im Sinne von beruflichen Umoder gar Neuorientierungen dar. Bisher wird es den Kundinnen und Kunden weitgehend selbst überlassen, für die Selektion und Passung der jeweiligen Weiterbildung für ihre berufliche Laufbahnplanung zu sorgen und die erforderlichen Kompetenzen zu erwerben. Eine explizite Standortbestimmung mit professioneller Unterstützung von Berufs- und Laufbahnberatenden ist eher selten oder gar nicht vorzufinden. Auch in der Phase der Durchführung einer Weiterbildung wird aktuell an Hochschulen nur vereinzelt und wenig systematisch auf den Bedarf nach Begleitung bei der persönlichen Lern- und Karriereentwicklung professionell reagiert. Es existiert kein umfassendes und fundiertes Konzept, das darauf ausgerichtete Beratungs- und/oder Coaching-Angebote beinhaltet.

Aus diesem Grunde haben vier Teilhochschulen der Fachhochschule Nordwestschweiz (FHNW) im Rahmen einer "strategischen Initiative« in vier Pilotprojekten innovative Coaching-Angebote für ihre Weiterbildungen durchgeführt und evaluiert. Coaching wird dabei als Ansatz zur Selbststeuerung und -regulation betrachtet, der die Reflexions- und Handlungskompetenz (Loebbert, 2013; Storch \& Kuhl, 2012; Tietgens, 1990) der Weiterbildungsteilnehmenden signifikant erhöht. Durch »Blended Coaching « werden präsenz- und mediengestützte Formate für ein One-to-one-Coaching mittels Chat, Application Sharing und Videoconferencing integriert, und es wird dem veränderten Kundenverhalten gegenüber neuen Medien Rechnung getragen (Anthony \& Nagel, 2012; Boos \& Rack, 2005; Clutterbuck \& Hussain, 2010; Cornelius, 2006; Eberling, 2010; Eberling, 2016; Geißler \& Metz, 2012; Geißler, 2016; Ziemons, 2012).

\section{Der Laufbahnkoffer HR in der Weiterbildung MAS HR-Management als Blended-Coaching-Tool}

In der professionellen Begleitung von beruflichen Transformationsphasen liegen Herausforderungen und Chancen, die zum Aufgabengebiet von internen HR-Professionals gehören. Coaching und Mentoring sind in vielen modernen Unternehmen integraler Bestandteil der Professionalisierungsstrategie (Böning, 2016; Haasen, 2001; Ulrich, Younger, Brockbank \& Ulrich, 2012). 
Die Gestaltung dieser »kritischen Übergänge« wird zum festen Bestandteil des "Transformationsmanagements«, zum Beispiel durch Fach-Coaching für bestimmte Berufsgruppen, durch Mentoring und Coaching im Talent-Management, im »Onboarding «, beim Übergang in die erste Führungsposition oder beim Transfer-Coaching im Rahmen von Trainings. Im Folgenden sollen die konkreten Tools des Laufbahnkoffers mit ihren Merkmalen und der Ansatz und Ablauf des Coachings genauer dargestellt werden.

Coaching ist eine Beratungsform, die das Ziel hat, anregende und teils ungewohnte Sichtweisen und neue Handlungsmöglichkeiten zu eröffnen. Ziel »ist die signifikante Verbesserung der Steuerung von Entscheidungen und Erfolgen beruflichen Handelns. Themen sind Verhaltensänderung, Umgang mit Krisen und Konflikten, wirkungsvolle Kommunikation und Rollengestaltung, berufliche und organisationale Veränderung, strategische Positionierung und Entwicklung, wirtschaftlicher Erfolg, Werte und Sinngebung (Loebbert, 2013). Wenn die beteiligten Personen durch längere Phasen von Unsicherheit und damit verbundener Instabilität gehen, in denen die individuelle Komfortzone (gesichertes Wissen, ausgebildete Kompetenzen, routiniertes und verlässliches Handeln) verlassen wird und in einem längeren Lernprozess durch das Neue ersetzt werden muss, sprechen wir von »Musterwechseln « im Gegensatz zu "Optimierungen" von bereits bestehenden Kompetenzen (Kruse, 2004).

Coaching im Rahmen des MAS HR-Management geschieht auf Grundlage einer Haltung der Ressourcenorientierung, Wertschätzung und Lösungsorientierung. Es folgt einem Modell der Komplementärberatung (Königswieser \& Hillebrand, 2004). Als Prozessberatung orientiert sich das Coaching am Veränderungsprozess des Coachee und unterstützt ihn, seine Situation selbst zu ändern. Als Fachberatung stellt es zusätzlich Wissen aus dem HR-Berufsfeld (durch einen HR-Experten) zur Verfügung und verbindet dies mit den Zielsetzungen der Coachees.

\section{Die Tools des Laufbahnkoffers HR}

$\mathrm{Zu}$ Beginn des Coachings im MAS HRM wird anhand eines onlinebasierten Tools, des Laufbahnkoffers HR, eine Standortbestimmung, eine Ressourcenanalyse und eine Zielbestimmung vorgenommen. Im Überblick werden die Elemente im Folgenden kurz skizziert. 


\section{Die Porträts}

Es werden für den Laufbahnteil drei bis vier Porträts präsentiert, die typische Veränderungsanliegen im Bereich der Laufbahnentwicklung HR betreffen wie zum Beispiel folgende:

- Die Person möchte sich in eine Leitungsstelle wie zum Beispiel Personalleitung entwickeln.

- Sie möchte sich aus einer Spezialistinnen- oder einer administrativen Funktion in die Funktion »Business Partner« entwickeln.

- Sie möchte eine Fachspezialistinnenkarriere (z. B. Talentmanagement) im HR einschlagen.

- Sie möchte sich aus einer Leitungsstelle heraus in die Leitungsverantwortung bei einer größeren Firma entwickeln.

\section{Die Standortbestimmung}

Mit diesem Tool können die Coachees ihre Berufs-, Arbeits- und Lebenssituation reflektieren. Sie erhalten ein Stimmungsbild zu ihrer momentanen Zufriedenheit im Berufsfeld. Es soll sie dazu anregen, jene Schritte zu unternehmen, die ihnen wichtig zur Verbesserung ihrer beruflichen Situation sind.

\section{Stärken-Schwächen-Profil}

Das Tool soll die Coachees unterstützen, ihre Stärken und Schwächen zu erkennen und damit ihre nächsten beruflichen Schritte zu planen. Sie überlegen sich, welche Tätigkeiten sie gerne und gut verrichten, welche Tätigkeiten ihnen schwerfallen und welche sie gerne verbessern möchten. Es entsteht ein Gesamtbild der Stärken und Schwächen, aus dem heraus konkrete Maßnahmen zur Weiterentwicklung definiert werden.

\section{Vision entwickeln}

Dieses Tool unterstützt die Entwicklung einer Vision für die eigene berufliche Zukunft. Unter »Vision" wird ein Bild von dem verstanden, was die Coachees im Innersten leitet, antreibt und motiviert. Auch wenn eine Vision nicht immer unmittelbar und zeitnah erreichbar ist, gibt sie in wichtigen Lebensfragen eine sinnvolle Richtung und wertvolle Orientierungen und sorgt häufig auch für die notwendige Energie, um Dinge in Angriff zu nehmen. 


\section{Der Ablauf des Coachings im Kurs}

Alle Teilnehmenden erhalten einen Internetzugang zum Laufbahnkoffer HR. Die Standortbestimmung wird von allen Teilnehmenden des Kurses ausgefüllt und mit den Peers nach einem Leitfaden besprochen. Dabei konkretisieren sich die persönlichen Anliegen und Ziele, Gemeinsamkeiten und Unterschiede werden deutlich. Häufig verfügen einige Peers über Wissen und Erfahrungen in Bereichen, in denen sich andere hineinentwickeln wollen. Die Teilnehmenden erhalten jeweils drei Coaching-Sitzungen als Einzelcoaching über Adobe-Connect oder Skype. Die Themenfelder und Ziele ergeben sich aus der mit dem Laufbahnkoffer durchgeführten Standortbestimmung. An einer Coaching-Sitzung nimmt dann auch ein Fachspezialist - in unserem Fall Gery Bruederlin als Leiter des MAS HRM - teil, um den fachspezifischen Teil zu beraten und die gewählte Richtung zu konkretisieren. Im Coaching-Prozess werden jeweils Aufgabenstellungen erarbeitet, die in der darauffolgenden Sitzung evaluiert werden. Der Verlauf wird mit geeigneten Methoden dokumentiert (durch Protokolle, Skalen, Tagebücher o. Ä.). Zum Abschluss des Coachings wird eine Evaluation des Gesamtprozesses durchgeführt.

\section{Nutzenanalyse nach den Pilotprojekten}

Die Auswertung der Pilotprojekte zeigte, dass die Teilnehmenden eine innovative Beratungsleistung in Form von Blended Coaching für ihre Lern- und Karriereentwicklung sehr schätzten und dies als integralen Bestandteil der Weiterbildung für wünschenswert erachteten (Abbildung 1).

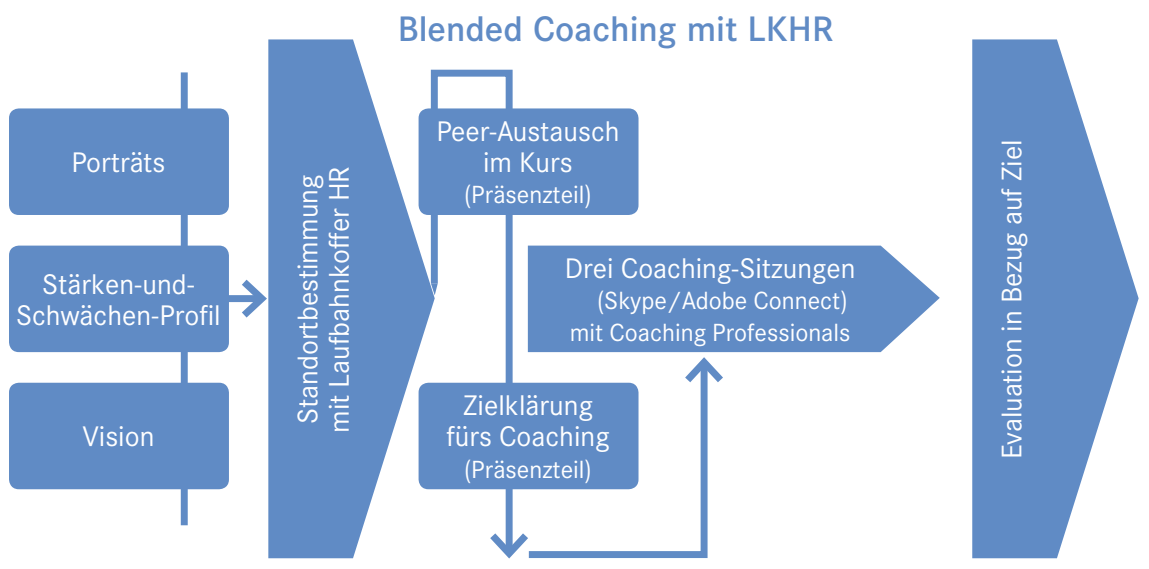

Abbildung 1: Blended Coaching mit dem Laufbahnkoffer HR 
Die kompetenzorientierte Anwendung des Stärken-Schwächen-Profils erlaubt es, individuell und passgenau eigene Entwicklungsbereiche zu identifizieren und im Verlauf des Kurses gezielt zu verbessern. Der Coaching-Prozess mit professioneller Unterstützung (Coach, Fachexperte) ermöglicht schon während der Weiterbildung den Erfahrungsaustausch mit Peers, eine weitergehende Zielklärung und erste Weichenstellungen in Richtung Zukunft, die zielgerichtete Entwicklung von Ressourcen und ein systematisches Prozessfeedback für die entwickelten Strategien.

\section{Der nächste Entwicklungsschritt für den Laufbahnkoffer:}

Spuren von Selbstwirksamkeit durch Real-Time-Monitoring sichtbar machen

In den letzten Jahren haben im therapeutischen Bereich neue Verfahren Licht in das Dunkel der »Black Box« von Veränderungsprozessen gebracht. »Was« und "Wie« werden durch prozessbegleitende Fragebögen sichtbar und von den Klientinnen und Klienten durch tägliche Selbstbewertungen entlang eines Kompetenzprofils erfahrbar gemacht. Dieses Synergetic Navigation System (SNS) ist ein internetbasiertes Verfahren zur Dokumentation, Messung, Analyse und Gestaltung von Veränderungsprozessen in komplexen Systemen (Dörhöfer \& Eberling, 2009; Dörhöfer \& Eberling, 2016; Eberling, 2010; Haken \& Schiepek, 2010; Schiepek, Eckert \& Kravanja, 2013; Schiersmann, Friesenhahn \& Wahl, 2015). Es geschieht real-time und ermöglicht dadurch den Handelnden eine unmittelbare und zeitnahe Überprüfung oder Korrektur ihrer Handlungssteuerung. Dies wiederum erhöht das Gefühl selbstwirksamen Handelns auf einer höheren Lernebene und verstärkt damit den nachhaltigen Einsatz persönlicher Erfolgsstrategien.

Coaching ist in diesem Sinne und nach unserem Selbstverständnis Ermöglichung von Selbstorganisationsprozessen, welche die Coachees befähigen, selbst gewählte Zielsetzungen mit eigenen Ressourcen zu verwirklichen. Der Laufbahnkoffer folgt demnächst dieser Veränderungslogik. Das bereits vorhandene Stärken-und-Schwächen-Profil wird um ein vom Coachee auszuwählendes Kompetenzen-Set (vgl. Bruederlin, 2014) ergänzt werden. Kompetenzentwicklung wird mittlerweile als Kerntreiber der HR-Leistungsfähigkeit angesehen (Ulrich et al., 2012; Bruederlin, 2014). Im Sinne eines Kompetenz-Monitorings können auf den persönlichen Entwicklungsfokus der HR-Person abgestimmte Fragebögen auf einem Server hinterlegt und von den Coachees in einem möglichst kurzen Rhythmus (etwa wöchentlich) eingeschätzt werden. Dadurch wird 
der Coaching-Prozess fortlaufend sowohl vom Coach als auch vom Coachee evaluiert und über die Bögen des Laufbahnkoffers wie ein Navigationssystem bis zur Zielerreichung benutzt.

Die Erweiterung des Laufbahnkoffers HR orientiert sich dabei inhaltlich an acht HR-Rollen, die das gesamte moderne HR-Leistungsspektrum abdecken (Abbildung 2):

- HR als strategieunabhängige, kostenfokussierte Organisation (Rollen 1 und 2),

- HR als strategieausführende, reaktive Organisation (Rollen 3 und 4),

- HR als strategieergänzende, kundenfokussierte Organisation (Rollen 5 und 6),

- HR als strategieintegrierende, wertschöpfende Organisation (Rollen 7 und 8).

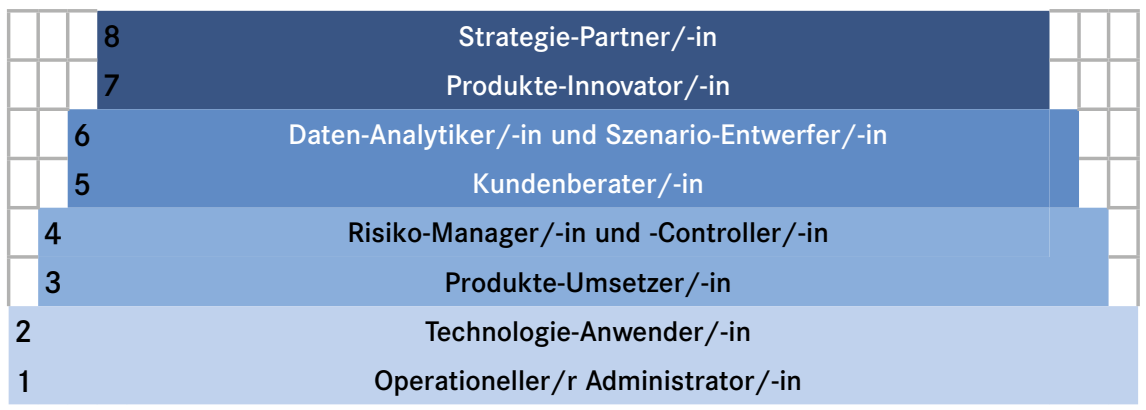

Abbildung 2: »HR-Rollenhierarchie»

Die Coachees können dann in Abstimmung mit der angestrebten Rolle aus dem Kompetenzprofil die zur Ausgestaltung der entsprechenden Rolle erforderlichen Kompetenzen, die durch detaillierte Items operationalisiert werden, auswählen und im Verlauf des Coachings mit Hilfe ihres Coaches über die Reflexion der eigenen Selbsteinschätzung und durch Einbezug von relevanten Fremdeinschätzungen gezielt verbessern (Tabelle 1). 
Tabelle 1: HR-Kompetenzenausprägung für die einzelnen hierarchischen HR-Rollen

\begin{tabular}{|c|c|c|c|c|c|}
\hline Kompetenzen & & Rollen 1 und 2 & Rollen 3 und 4 & Rollen 5 und 6 & Rollen 7 und 8 \\
\hline \multirow{2}{*}{\multicolumn{6}{|c|}{ Fachlich }} \\
\hline & & & & & \\
\hline \multirow[t]{5}{*}{ Betriebswirtschaftlich } & Finanz & & & & \\
\hline & Marketing & & & & \\
\hline & Organisation & & & & \\
\hline & Kommunikation & & & & \\
\hline & Beschaffung & & & & \\
\hline & & & & & \\
\hline \multirow[t]{9}{*}{ Funktional/HR-spezifisch } & Kernkompetenzen & & & & \\
\hline & (HR Life-Cycle) & & & & \\
\hline & & & & & \\
\hline & Pragmatismus & & & & \\
\hline & Analytische Fähigkeiten & & & & \\
\hline & Konzeptionsstärke & & & & \\
\hline & & & & & \\
\hline & Innovative Trendbereiche & & & & \\
\hline & (z.B. HR-Analytics) & & & & \\
\hline & & & & & \\
\hline \multirow[t]{6}{*}{ Sozial-Kommunikativ } & Kundenorientierung & & & & \\
\hline & Problemlösungsfähigkeit & & & & \\
\hline & Beratung & & & & \\
\hline & Anpassungsfähigkeit/Flexibilität & & & & \\
\hline & Verhandlungsgeschick/Konfliklösu & & & & \\
\hline & & & & & \\
\hline \multirow[t]{5}{*}{ Handlungsbezogen } & Initiative & & & & \\
\hline & Erfolgsorientierung & & & & \\
\hline & Schlagfertigkeit & & & & \\
\hline & Einflussnahme/Überzeugungskraft & & & & \\
\hline & Durchsetzungskraft & & & & \\
\hline & & & & & \\
\hline \multirow[t]{4}{*}{ Personal } & Motivation/Einstellung & & & & \\
\hline & Lernbereitschaft & & & & \\
\hline & Glaubwürdigkeit & & & & \\
\hline & Ganzheitliches Denken & & & & \\
\hline
\end{tabular}

\section{Der nächste Entwicklungsschritt für die Coaches:}

Erhöhung der bewussten Handlungssteuerung der Coaches durch die gezielte Verwendung der acht generischen Prinzipien nach Günter Schiepek

Während für die Coachees durch die Verwendung der an Kompetenzprofilen orientierten Selbsteinschätzungsbögen die bewusste Handlungssteuerung erhöht wird, erfolgt das auf Coach-Seite durch die Verwendung der acht generischen Prinzipien von Schiepek, Eckert \& Kravanja (2013). Diese stellen Leitlinien für unser Orientierungs- und Handlungswissen als Coaches dar. Sie ermöglichen uns als Coaches ein bewussteres Gestalten der kritischen Übergänge, die in jedem Coaching-Prozess zu finden sind.

Im Einzelnen umfassen sie folgende Schritte, deren Reihenfolge aber nicht strikte eingehalten werden muss. 


\section{Prinzip 1}

Unsicherheit und Ungewissheit in Bezug auf die angestrebten Veränderungen bringen Coachees häufig in einen Zustand von »Instabilität«. Stabilität für Veränderungsprozesse unter faktischen Instabilitätsbedingungen zu schaffen, wird zur wichtigsten Gestaltungsaufgabe. Emotionale Sicherheit muss deswegen erzeugt werden durch Transparenz von eigenen Visionen und Zielsetzungen, Vertrauen in sich und andere (Selbstwert-Unterstützung), Planungssicherheit trotz Unsicherheit und begrenzter Vorhersage-Horizonte, Verdeutlichung und Aktivierung von vorhandenen Ressourcen, durch die Erfahrung von Selbstwirksamkeit (»ich kann/wir können etwas bewegen und zustande bringen«) und das Einrichten von Experimentierfeldern und Pilotprojekten.

\section{Prinzip 2}

An der Entstehung und der Auflösung von Problemsituationen sind häufig verschiedene und unterschiedliche Personen beteiligt. Muster des relevanten Systems zu identifizieren, hilft deshalb, das »systemische Feld« zu begreifen und darin angemessen zu agieren. Um wirksame Muster zu identifizieren, helfen folgende Fragen: Auf welche Systemteile zielt Veränderung ab? Was muss weshalb verändert werden? Wer muss unbedingt einbezogen werden, wer nicht? Danach erst gestalten die Coachees ihre Beziehungen zu den Beteiligten. Die computerbasierte Prozesserfassung erfolgt unmittelbar und zeitnah, was die Erfahrung von Selbstwirksamkeit und damit eine zunehmende Selbststeuerung ermöglicht.

\section{Prinzip 3}

Häufig sind Veränderungswünsche Anlass für Coaching. Diese werden aber nicht immer von allen Beteiligten auf gleiche Weise geteilt (Chefs sehen zum Beispiel Dinge häufig anders als ihre Mitarbeitenden). Coaching-Prozesse sollten deswegen immer darauf ausgerichtet werden, den "Sinnbezug zu ermöglichen «, also den »Sinn « hinter den Wünschen zu erkennen und zu vermitteln. Häufig gelingt dies, wenn die Prozesse mit den wichtigsten eigenen Zielvorstellungen und Lebenskonzepten der Coachees in Verbindung gebracht werden können. Dazu verhelfen Fragen wie: Welche Möglichkeiten eröffnen diese Veränderungsprozesse für mich? Welchen Nutzen haben sie für das Unternehmen und mich? Daraus ergibt sich, dass die Beteiligten in Planungs- und Entwicklungsprozesse umfassend und möglichst frühzeitig einbezogen werden und dass die erhofften Vorteile und Möglichkeiten aufgezeigt werden sollten. 


\section{Prinzip 4}

Veränderungsprozesse benötigen wie jede Bewegung Energie. Energetische Aktivierung (»Motivation«) entsteht besonders leicht über die Berücksichtigung und Entwicklung der eigenen Ressourcen (»Selbstwirksamkeit erfahren ", "Stolz auf eigene Ideen wecken«) und durch Bezug zu den Werten der Beteiligten, was für diese eine hohe emotionale und motivationale Bedeutung schafft. Das verstehen wir unter »Kontrollparameter aktivieren«.

\section{Prinzip 5}

Um sich tatsächlich zu verändern, müssen Coachees "destabilisiert «, also aus ihrer bisherigen Komfortzone (»die bisherige Routine«, »eingespielte Muster«) gebracht werden. Die Antizipation von Krisen und herausfordernde Zukunftsbilder sind dazu geeignet. Auf der Umsetzungsebene können dafür Pilotprojekte, Verhaltensexperimente, Systemspiele, Benchmarking, Musterunterbrechungen, veränderte Symbole oder Forcierung und Bestärkung vorhandener Entwicklungsprojekte eingesetzt werden (Haken \& Schiepek, 2010; Schiepek et al., 2013). Im Prinzip geht es also um Destabilisierung einerseits und um Fluktuationsverstärkung (»die Ausnahme zur Regel machen«) andererseits.

\section{Prinzip 6}

Es gilt, die richtigen Zeitpunkte (kairos) zu nutzen und Synchronisation herzustellen. Jeder Coach muss sich für geeignete Zeitpunkte sensibilisieren und die richtigen Momente erwischen, er muss deshalb auf die Aufnahmebereitschaft von Coachees achten. Dabei hilft ihm, wenn er den Informationsfluss situationsgerecht organisiert und plankomplementäres Verhalten zeigt (»Ich halte mich an das, was ich geplant habe «). Oft sind auch Prozesse abzustimmen, Zeitrituale einzuführen und einzuhalten und ist regelmäßiges Ergebnisfeedback zu geben.

\section{Prinzip 7}

Es gilt ferner, gezielte Symmetriebrechung zu ermöglichen. Im Zustand von großer (»kritischer«) Instabilität können sich mehrere Zustände eines Systems gleichzeitig verwirklichen, wie man es von Kippbildern (»alte Frau«/»junge Frau«, »Vase «/»Gesicht«) kennt. Das führt häufig zu Irritationen und Entscheidungs- und Verhaltensunsicherheiten. Sinnvolle Weichenstellungen durch kleine Verstärkungen erwünschter Muster oder die bewusste Bevorzugung bestimmter 
Tendenzen eignen sich dann dazu, Eindeutigkeit herzustellen. Auch kann die detaillierte Fokussierung und Ausgestaltung eines neuen Musters hilfreich sein.

\section{Prinzip 8}

Alle neuen und erwünschten Muster müssen wieder stabilisiert, automatisiert und routinemäßig verfügbar gemacht werden - also müssen wir hier als Coaches Restabilisierung etablieren. Psychologisch gesprochen, werden die neuen Muster in bestehende kognitiv-emotionale Schemata integriert oder damit vernetzt.

\section{Fazit}

»Blended Coaching mit dem Laufbahnkoffer $\mathrm{HR}$ « stellt eine neue Art des "ressourcenorientierten und kompetenzbasierten « Coachings dar, das in der Weiterbildung den Kursteilnehmenden eine gezielte Gestaltung des Überganges ermöglicht. Nach den Grundannahmen eines ressourcen- und lösungsorientierten Ansatzes (Bamberger, 2001; Eberling, 2016; Storch \& Kuhl, 2012) steht die Erhöhung der Selbstreflexions- und Selbststeuerungskompetenz im Mittelpunkt, die durch den Laufbahnkoffer HR maßgeblich gefördert wird (Greif, 2016). Durch die geplanten Weiterentwicklungen anhand eines Kompetenzprofils und die Verwendung der acht generischen Prinzipien nach Schiepek erhält der Laufbahnkoffer HR Anschluss an moderne Entwicklungen wie die des Real-Time-Monitorings, die eine neue und vielversprechende Richtung einer evidenzbasierten wissenschaftlichen Forschung darstellen. Zugleich wird damit Orientierungs- und Handlungswissen für eine immer schneller und komplexer werdende Welt (Guwak \& Stroltz, 2012) bereitgestellt.

\section{Literatur}

Anthony, K., Nagel, D. A. (2012). A brave new world: coaching online. Coaching Today, 33 (1), 33-37. Bamberger, G. (2001). Lösungsorientierte Beratung. Praxishandbuch (2., völlig neu bearb. Aufl.). Weinheim: Beltz.

Böning, U. (2016). Business-Coaching mit Führungskräften. In R. Wegener, M. Loebbert, A. Fritze (Hrsg.), Coaching-Praxisfelder. Forschung und Praxis im Dialog (2. Aufl., S. 11-31). Wiesbaden: Springer.

Boos, M., Rack, O. (2005). Gestaltung netzbasierter Kollaboration. Arbeiten und Lernen in Gruppen. In D. Euler, S. Seufert (Hrsg.), E-Learning in Hochschulen und Bildungszentren (S. 281298). München: Oldenbourg. 
Bruederlin, G. (2014). Die leistungsfähige HR-Funktion: Kompetenzen, Strategie und Struktur. In HR-Jahrbuch 2014 (S. 45-76). Zürich: Weka.

Clutterbuck, D., Hussain, Z. (2010). Virtual Coach, Virtual Mentor. Charlotte, N.C.: Information Age Publishing.

Cornelius, C. (2006). Entwicklung und Evaluation des Online-Coaching - »Zeit und Ziele für Wissenschaftlerinnen«. In J. Dalhoff (Hrsg.), Anstoß zum Aufstieg. Karrieretraining für Wissenschaftlerinnen auf dem Prüfstand (S. 95-103). Bielefeld: Kleine.

Dörhöfer, S., Eberling, W. (2009). Wissensaustausch sichtbar machen. Wissensmanagement, 11 (6), 42-44.

Dörhöfer, S., Eberling, W. (2016). Future Perfect Inc. - Coaching-Kultur 2020. In R. Wegener, M. Loebbert, A. Fritze (Hrsg.), Zur Differenzierung von Handlungsfeldern im Coaching. Die Etablierung neuer Praxisfelder (S. 499-510). Wiesbaden: Springer VS.

Eberling, W. (2010). Real-time Monitoring durch SNS und Interaktionsmatrix: Mit evidenzbasierten Tools auf dem Weg zum Netzwerk-Management und zur Netzwerk-Intelligenz? WEKA online.

Eberling, W. (2016). Supervision und Coaching. In M. Dick, W. Marotzki, H. Mieg (Hrsg.), Handbuch Professionsentwicklung (S. 283-294). Bad Heilbrunn: Klinkhardt.

Geißler, H. (2016). Coaching mit modernen Medien - Produktdiversifizierung und Erschließung neuer Marktsegmente durch Coaching mit modernen Medien. In R. Wegener, M. Loebbert, A. Fritze (Hrsg.), Zur Differenzierung von Handlungsfeldern im Coaching. Die Etablierung neuer Praxisfelder (S. 273-283). Wiesbaden: Springer VS.

Geißler, H., Metz, M. (2012). E-Coaching und Online-Beratung. Wiesbaden: Springer VS.

Greif, S. (2016): Wie wirksam ist Coaching? Ein umfassendes Evaluationsmodell - für Praxis und Forschung. In R. Wegener, M. Loebbert, A. Fritze (Hrsg.), Coaching-Praxisfelder. Forschung und Praxis im Dialog (2. Aufl., S. 161-182). Wiesbaden: Springer.

Guwak, B., Stroltz, M. (2012). Die vierte Kränkung: Wie wir uns in einer chaotischen Welt zurechtfinden. Wien: Goldeck.

Haasen, N. (2001). Mentoring - Persönliche Karriereförderung als Erfolgskonzept. München: Heine.

Haken, H., Schiepek, G. (2010). Synergetik in der Psychologie. Selbstorganisation verstehen und gestalten (2., korr. Aufl.). Göttingen: Hogrefe.

Königswieser, R., Hillebrand, M. (2004). Einführung in die systemische Organisationsberatung. Heidelberg: Carl-Auer-Verlag.

Kruse, P. (2004). Next Practice. Erfolgreiches Management von Instabilität. Wiesbaden: Gabal.

Loebbert, M. (Hrsg.) (2013). Professional Coaching. Konzepte, Instrumente, Anwendungsfelder. Stuttgart: Schäffer-Poeschel.

Schiepek, G., Eckert, H., Kravanja, B. (2013). Grundlagen systemischer Therapie und Beratung. Göttingen: Hogrefe.

Schiersmann, C., Friesenhahn, J., Wahl, A. (2015). Synergetisch beraten. Göttingen: Hogrefe.

Storch, M., Kuhl, J. (2012). Die Kraft aus dem Selbst. Sieben PsychoGyms für das Unbewusste. Bern: Huber.

Tietgens, H. (1990). Vorbemerkungen. In E. Fuchs-Brüninghoff (Hrsg.), Supervision in der Erwachsenenbildung (S. 15-17). Bonn: DIE.

Ulrich, D., Younger, J., Brockbank, W., Ulrich, M. (2012). HR from the Outside In. Six competencies for the future of human resources. New York: McGraw-Hill.

Ziemons, M. (2012). Blended Business Coaching. Organisationsberatung, Supervision, Coaching, $19(2), 217-225$. 


\section{Kein Coaching ohne Evaluation der Ergebnisse}

Niki Harramach und Nina Veličković

In der Coaching-Theorie werden unterschiedliche Qualitätsdimensionen definiert und erforscht: Strukturen, Prozesse und Ergebnisse. In der Praxis kennen Kunden und Kundinnen aber nur eine Qualität: Ergebnisse - dass die vereinbarten Ziele erreicht wurden. Die anderen Dimensionen sind aus Kundensicht nur Faktoren, die Qualität ermöglichen - also Mittel, nicht Ziele.

Auf der Basis von über dreißig Jahren Wirtschaftscoaching mit mehr als 500 Fällen stellen wir den derzeitigen Stand der Evaluationstechnik aus unserer Sicht kritisch dar. Wir zeigen, wie wir erfolgreiches Coaching sicherstellen und welche Rolle dabei dem Benchmarking zukommt. Last but not least wollen wir mit diesem Beitrag aus der Sicht der Praxis einen spezifischen Dialog mit Wissenschaft und Forschung einleiten.

\section{Vorbemerkungen}

$\mathrm{Zu}$ Beginn wollen wir unser Verständnis des Kundenbegriffs, von Rahmenbedingungen und Ablauf von Coachings und unsere Qualitätskriterien für Coaching den gängigen wissenschaftlichen Modellen gegenüberstellen.

Wirtschaftscoaching nennen wir in Österreich jenen Bereich von Coaching, der im Rahmen von Wirtschaftsunternehmen und Non-Profit-Organisationen stattfindet. Die Ausgangsbedingungen sind zum überwiegenden Teil wie folgt: Die Organisation bezahlt Coachings für ihre Mitarbeitenden und stellt diese in der Arbeitszeit dafür frei. Der Coach wird vom Coachee ausgesucht - entweder in freier Wahl oder aus einem Pool, der von der Organisation bestimmt wird. In der Abwicklung 1 besteht daher ein Dreiecksverhältnis zwischen Coach, Auftraggeber in der Organisation (Kunde) und Klient/-in (Coachee) (vgl. Abbildung 1). 


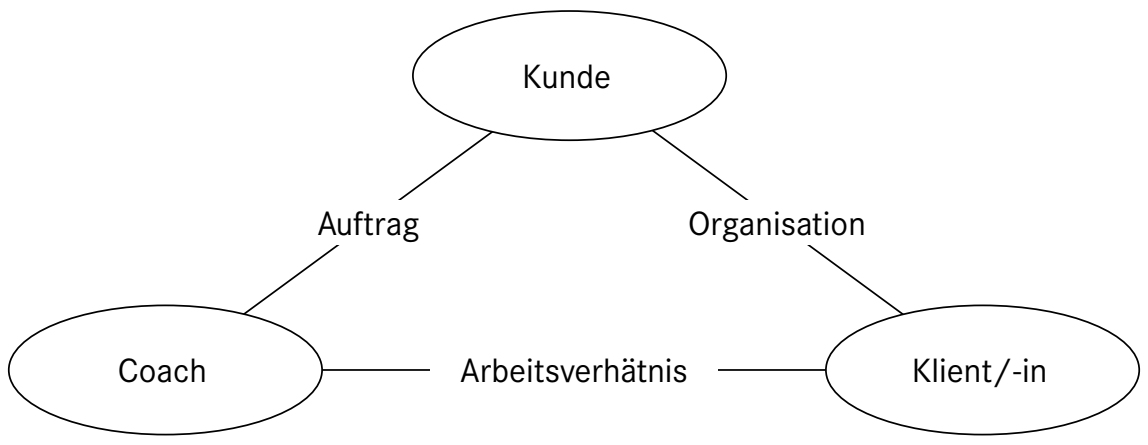

Abbildung 1: Das Wirtschaftscoaching-Dreieck Coach - Kunde/Auftraggeber - Klient/-in

Ziele und Inhalte des Coachings werden, im Rahmen der Aufgaben der Klientin oder des Klienten in der Organisation, zwischen Coach und Klient/-in vereinbart. Methoden und Vorgehensweise werden in Abstimmung mit dem Klienten oder der Klientin professionellerweise vom Coach bestimmt. Die Dauer ist mit einer Obergrenze (oft zehn Einheiten von 45 oder 50 Minuten) fixiert. Sie ist oft noch kürzer, manchmal länger - weil sie durch die Zielerreichung bestimmt wird. Nach Ende des Coachings erfolgt ein Abschlussbericht an die Auftraggeber, der zwingend dem Datenschutz genügen muss.

\section{$Q$ wenn $E=A$}

Qualität liegt vor, wenn die Anforderungen des Kunden eine gehörige Erfüllung gefunden haben; wenn also die Erfüllung den Anforderungen entspricht. Wir wagen dazu eine Formel: Q wenn E = A. Dieser Ansatz liegt allen gängigen Qualitätsnormen zugrunde. Es ist auch juristisch geboten, sich daran zu halten. Wenn keine gehörige Erfüllung vorliegt, bestehen rechtlich Leistungsansprüche des Kunden, wie zum Beispiel Gewährleistung und Schadenersatz. Dass es im Bereich von Training und Coaching derzeit noch wenig Rechtspraxis gibt, darf nicht darüber hinwegtäuschen, dass wir erstens auch in dieser Profession den Rechtsnormen unterliegen und zweitens rechtliche Durchsetzung sich mit steigender Quantität und Qualität mehren wird. Aus diesem Grund gibt es auf die Frage von Geißler (vgl. Geißler \& Wegener, 2015, S. 9): »Wer sollte denn eigentlich bei der Bewertung von Coaching-Prozessen das erste und wer das letzte Wort haben?«, nur eine Antwort: Es ist weder »die Praxis« noch »die Wissenschaft», es sind die Kundinnen und Kunden.

Nach 2000 hat sich in der Literatur zu Evaluation von Coaching ein »state of the art « uniformiert. Es wird vor allem unterschieden und Bezug genommen 
auf drei spezifische Dimensionen von Qualität (Heß \& Roth, 2001; Bischof, 2011; Runde, 2016).

- Strukturqualität: Dazu zählen personelle Faktoren der Coaches wie ihre Qualifikation, Kompetenz; ferner personelle Faktoren der Klientinnen und Klienten wie Bereitschaft zu Veränderung und Mitarbeit; Faktoren der Beziehung von Coach und Klient/-in wie Passung, Vertrauen; Unternehmensfaktoren wie die Bereitschaft, ergebnisoffen zu arbeiten.

- Prozessqualität: Dazu gehören beispielsweise Faktoren wie Auftragsklärung, Sitzungsgestaltung, Methodenpassung, Prozessreflexion, Umsetzungsunterstützung.

- Ergebnisqualität: Dazu werden genannt Zufriedenheit, Zielerreichung, Änderung von Verhaltensweisen, von Einstellungen.

Die Kundinnen und Kunden - im Dreieck von Abbildung 1 sowohl Klienten und Klientinnen als auch Auftraggeberinnen und Auftraggeber - sind indessen nur am Ergebnis interessiert. Der Begriff Ergebnisqualität ist daher aus der Kundensicht eine verwirrende Tautologie und insgesamt irreführend: Eine andere Qualität gibt es nicht. Wirkfaktoren wie Strukturen und Prozesse sind aus Kundensicht keine Ergebnisse. Wohl aber sind sie Gütekriterien und (Miss-)Erfolgsfaktoren für Qualität. Sie spiegeln den Stand der Technik wider. Alle, die sich als Coach ausgeben und anbieten, haften (auch juristisch - in Österreich nach $\$ 1299$ des Allgemeinen Bürgerlichen Gesetzbuchs) dafür, den diesem Stand der Technik entsprechenden Sachverstand zu haben. Insofern ist es wichtig zu untersuchen, inwieweit solche Wirkfaktoren das Ergebnis von Coachings beeinflussen. Aber das ist nicht der primäre Gegenstand von Evaluation.

Im Folgenden wollen wir daher darstellen, wie wir seit 1984 in dem Format, das man heute "Coaching " nennt, vorgehen und dabei die Ergebnisse evaluieren. Wir zeigen ferner, wie die Methode Benchmarking zur Qualitätssicherung eingesetzt wird. Zum Schluss werden wir die Diskrepanz zwischen dem, was Wissenschaft und Forschung entwickelt hat, und dem, was die Praxis benötigt, beleuchten und Wissenschaft und Forschung um adäquatere Unterstützung zur Erreichung der Kundenziele ersuchen. Wir versuchen so, mit diesem Beitrag einen Dialog zwischen Praxis und Theorie einzuleiten. 


\section{Coaching und Evaluation konkret}

\subsection{Unsere Vorgehensweise}

Als wir 1984 mit der Unterstützung von Führungskräften in einer Art begannen, die man heute als »Coaching « bezeichnet, war die Kundschaft dafür rar. Umso wichtiger war es, die Wirksamkeit unserer Methode durch erkennbare Erfolge zu belegen. Diese Pionierzeit hatte bestimmte Spezifitäten: Vor 25 Jahren gab es den Begriff "Coaching « im deutschsprachigen Raum in der Wirtschaftswelt noch nicht. Das, was heute als "Coaching « bezeichnet wird, war damals Teil der Beratung. Den Klientinnen und Klienten wurde in Einzelgesprächen geholfen, Lösungen für ihre Probleme zu finden. Beratende achteten nicht so sehr darauf, ob sie das über Ratschläge erreichten oder dadurch, dass man die Kunden und Kundinnen unterstützte, selbst Lösungen zu finden. Diese beiden Möglichkeiten waren unreflektiert vermischt. Hauptsache war für die Beteiligten, dass am Ende die Kunden und Kundinnen ihr Problem erfolgreich gelöst hatten. Wenn es notwendig schien - und wenn Beratende dazu überhaupt in der Lage waren -, übte man mit den Kundinnen und Kunden auch und nannte es »Training «. Für Verhaltensänderungen wurden Rollenspiele geübt, diese galten damals als modern. Bei angestrebten Verhaltensmodifikationen glich also die Unternehmensberatung dem Coaching, wie es damals aus dem Sport bekannt war.

Durch eine Vergrößerung des Marktes (beim Angebot wie bei der Nachfrage) musste es auch zu einer Professionalisierung im Sinne eines definierten Standes der Technik kommen. Ein eigener Aus- und Weiterbildungsmarkt mit strukturierten Schulungsangeboten entstand. Berufsverbände und Standespolitik etablierten sich.

Ein paar Tugenden aus der Pionierzeit sollten wir uns aber bewahren, sollten sie sogar wieder einführen. Wir plädieren dafür, dass Coaches sich wieder auf die aus Sicht der Kundinnen und Kunden einzig wichtige Qualität konzentrieren, den Erfolg. Damit könnte mehr Kapazität und Flexibilität bei den Coaches für die Bedürfnisse der Kunden und Kundinnen eröffnet werden. Coaches sollten sich die Wege zum Erfolg freihalten. Das heißt sehr wohl, Wirkfaktoren zu identifizieren, aber sie dürfen nicht mit Ergebnissen verwechselt werden. So sollte es beispielsweise der Entscheidung der Coaches obliegen, ob sie den Coachees einen Ratschlag anbieten oder sie in der eigenen Lösungsentwicklung begleiten. Coaches sollten standespolitisch darauf achten, dass der »Ausbildungsmarkt « nicht den »Anwendungsmarkt« überflügelt. Die Qualität wird momentan verstärkt über Methoden definiert, Professionalität wird mit Qualifikation ver- 
bunden und nicht mit Kundenerfolg. Berufsverbände werden zu Ausbildungsverbänden. Der gesamte Markt - auch die Nachfrageseite - wird dadurch entscheidend beeinflusst. Auch Kundinnen oder Kunden beginnen, sich mehr an Ausbildungsnachweisen zu orientieren statt an Erfolgsnachweisen. Genau das ist hier unser Thema. Coaches sollten zudem mit Selbstreflexion und Selbsteinschätzung ihren Blick dafür schärfen, ob im gegebenen Fall Coaching überhaupt das richtige Format ist und ob der Coach über die geeigneten Kompetenzen zur Erreichung der vereinbarten Ziele im gegebenen Kontext verfügt. Denn ein Überangebot von Coaches führt nicht nur zu marktwirtschaftlich reinigendem Wettbewerb, sondern - insbesondere in relativ intransparenten Märkten - auch zu Qualitätsverlust.

Juristisch gesehen, gibt es auch eine Aufklärungspflicht der Coaches als Sachverständige auf ihrem Gebiet. Sollten sie erkennen (müssen), dass die Kundinnen und Kunden mangels Sachverstand nicht wissen, was im Coaching möglich ist und was nicht, haben sie professionell aufzuklären. Diese Pflicht zur sogenannten Meta-Beratung hat entscheidende Bedeutung für unser Thema der Evaluation. Sie markiert auch, wo das Prinzip »Hilfe zur Selbsthilfe endet. Ich darf mich als Coach nicht darauf verlassen, dass die Klienten und Klientinnen schon selber wissen werden, ob Coaching überhaupt die richtige Methode ist. Auch im gesamten Coaching-Prozess trifft die Coaches eine erhöhte Sorgfaltspflicht dafür, dass die Coaching-Schritte am richtigen Weg zur Zielerreichung liegen. Dafür müssen sie immer das vereinbarte Endergebnis im Auge haben. Und nichts hilft da mehr als die Evaluation der Ergebnisse - und das während des gesamten Coaching-Prozesses bestehende Bewusstsein, dass dies am Ende auch zwingend der Fall sein wird. Die zu Recht immer wieder beschworene Professionalisierung des Coachings wird entscheidend davon abhängen, ob und inwieweit es der Branche gelingen wird, ihren Beitrag zum Erfolg der Kundinnen und Kunden auszuweisen.

\subsection{Benchmarking}

Wie lässt sich feststellen, ob ein Training ein Erfolg (gewesen) ist? Dafür hat im Trainingsbereich bereits 1977 Donald L. Kirkpatrick sein Modell »The Four Levels" geschaffen. Er schlug vor, den Trainingserfolg wenn möglich auf vier Ebenen zu evaluieren: Reaction - Learning - Behavior - Results. Ähnlich, aber vereinfacht, unterscheidet Niki Harramach (1995) im Drei-Ebenen-Modell: Lernerfolge, Umsetzungserfolge und Organisationserfolge. Er empfiehlt, besonders auf Umsetzungserfolge zu fokussieren, und betont, dass jede Messung - auch von Misserfolgen - klare Ziele voraussetze. 
Beide Modelle sind auch für die Evaluation von Coaching anwendbar, weil die Output-Kontrolle im Zentrum steht. Kontrolle und damit Bewertung kann zuweilen quantitativ erfolgen, etwa bei Zielen wie der Beschleunigung von Prozessen oder einer angestrebten Reduktion von Kundenbeschwerden. Dagegen werden Vertrauen und Respekt nicht in Euro bewertet, da braucht es angemessene Wertmaßstäbe.

\section{Fallbeispiel A »Frei Sprechen»}

Eine Topmanagerin kommt mit dem Problem, sie könne vor einem größeren Auditorium nicht gut frei sprechen, das heißt extemporieren, improvisieren, auf Unvorbereitetes adäquat reagieren. Ihr Ziel ist, dies besser zu können. Das Coaching wird von der Organisation finanziert, das Ziel daher mit ihrem Chef akkordiert. Methode: Sie muss in einer U-Bahn-Station, immer wenn eine U-Bahn-Garnitur einfährt und die Leute aussteigen, über ein ihr vom Coach zugerufenes Thema sprechen. Das muss naturgemäß schnell gehen. Sie muss Reaktionen, auf die sie sich nicht vorbereiten kann, adäquat begegnen. Erfolgskontrolle: Nach drei Monaten wird evaluiert, wie die Klientin selbst ihre Verbesserung in dieser Hinsicht einschätzt (70 Prozent) und welchen Wert ihr Vorgesetzter nennt, der unser Auftraggeber ist (90 Prozent). Die Prozentzahlen wurden vom Coach in getrennter Befragung erhoben.

\section{Fallbeispiel B ॥Marktleiter»}

Einem Marktmanager-Stellvertreter eines großen Handelsunternehmens wird von seinem Personalchef ein Coaching empfohlen, um auf die Stelle des Marktleiters vorzurücken. Die Ziele, die laut dem Personalchef und dem Marktleiter nötig sind, um diese Aufstufung zu erreichen, werden von diesen vorgeschlagen und vom Coach mit dem Klienten - allenfalls nach Adaptierung - vereinbart: Der Klient muss klarer und öfter delegieren, die Umsetzung der Ziele auch kontrollieren und seine Konfliktfähigkeit erhöhen. Methode: Um die zwischen dem Kunden, dem Klienten und dem Coach abgestimmten Ziele zu erreichen, wird mit Schema-Coaching und Rollenspielen gearbeitet. Der Klient analysiert gemäß einem Schemafragebogen (Erhebung früher maladaptiver Schemata aus Migge, 2013) seine Handlungsmuster. Dadurch kann er in Zukunft erkennen, wann seine Schemata in beruflichen Situationen aktiviert werden, und bewusst anders denken und handeln. In einer Schema-Imagination werden eine Konfliktsituation bearbeitet und neue Handlungsalternativen entwickelt und im Rollenspiel gefestigt. Erfolgskontrolle: Selbsteinschätzung des Klienten, inwieweit er diese Fähigkeiten verbessert hat. Der Selbsteinschätzung ging ein Feedbackgespräch mit seinem Marktleiter voraus: Zielerreichung 100 Prozent. Explizit 
wurde hier nur die Abstimmung mit dem Marktleiter empfohlen, um die Kontrolle einfach zu halten. Auch noch die Mitarbeitenden des Klienten zu befragen (360-Grad-Feedback), schien uns desavouierend für den Klienten. Nach einem halben Jahr rückt der Klient tatsächlich in die Marktleiter-Position vor. Das finale Ziel wurde daher auch aus Sicht des Kunden zu 100 Prozent erreicht.

In diesen - und in allen unseren - Fällen ging und geht es uns darum, Evaluation so einfach wie möglich zu halten, um die Wirksamkeit von Coaching festzustellen. Das beginnt mit klaren operativen Zielen und endet mit möglichst einfachen Methoden der Evaluierung. Die Praxis braucht die größtmögliche valide Einfachheit. Erstaunlich ist, dass sich die ersten Versuche der Evaluation von Coaching nicht diese Modelle der Trainings-Erfolgs-Kontrolle zunutze gemacht, sondern das Rad neu erfunden haben. Die Begründungen dafür halten wir nicht für stichhaltig. So stellte Greif (2016) zu Kirkpatrick (1977) lediglich fest:

"Da seine Unterscheidungen allerdings eher pragmatisch sind und nicht dem Stand der Forschung entsprechen, hat er im strengen Sinne kein swissenschaftliches Modell entwickelt. Die Kriterien werden nicht eindeutig definiert, und Kirkpatrick hat keine Annahmen über ihre erwarteten Zusammenhänge formuliert. Außerdem fehlen Annahmen über Voraussetzungen und Bedingungen« (ebd., S. 166).

Das alles stimmt, verkennt aber Zweck und Charakter des Modells und kann wohl nicht als »kritisch«, eher nur als verständnislos gelten. Annahmen über Zusammenhänge, Voraussetzungen und Bedingungen sind nicht Gegenstand von Kirkpatricks Modell. Und was heißt in so komplexen Systemen wie Menschensystemen schon Wissenschaftlichkeit im strengen Sinne? Die viel zu opulenten Evaluationsmodelle, die seit Beginn dieses Jahrhunderts im Coaching entwickelt wurden, sind eher hinderlich als hilfreich. Sie unterscheiden nicht zwischen Ursachen, Ergebnissen/Zielen und Folgewirkungen.

Die Evaluation von Coaching muss sich wohl auf die Bewertung der Zielerreichung konzentrieren. Anderes ist nicht empfehlenswert und juristisch auch nicht zulässig. Der Zweck von Benchmarking ist nämlich erstens, die erfolgreichsten Projekte anhand der Zielerreichung zu identifizieren, zweitens festzustellen, welche best practices diese Projekte so erfolgreich gemacht haben, und drittens herauszufinden, was man von ihnen generell für andere Projekte lernen kann.

So wird bei McDonald's jeden Monat das Lokal mit den besten Ergebnissen ermittelt. Und alle anderen schauen sich davon ab, was sie ähnlich verbessern könnten. Auch die theoriegeleitete Coaching-Forschung möge den Prinzipien 
des Benchmarking folgen. Bei erwiesenermaßen erfolgreichen oder erfolglosen Coachings sollte dann untersucht werden, welche Wirkfaktoren zum Erfolg, welche zum Misserfolg - und in welchem Ausmaß - beigetragen haben. So könnte ein wissenschaftlich fundierter Katalog von Gütekriterien und damit ein einzuhaltender Stand der Technik definiert werden.

Aus solchen Analysen haben sich drei Kernschritte als relevant für den Erfolg herausgeschält:

- Ziele professionell definieren

Das Wichtigste dabei ist, die Ziele so zu definieren, dass klar ist, wer wann und wie kontrollieren kann, inwieweit sie erreicht worden sind. Auch hier ist Sachverstand gefragt, was im Coaching möglich und zulässig ist (beispielsweise Reduktion der Kundenbeschwerden bei einem Dienstleistungsunternehmen) und was nicht (beispielsweise Behandlung einer Angststörung). Zudem ist es Aufgabe der Coaches, die Klientinnen und Klienten bei der Operationalisierung ihrer Ziele zu unterstützen. Unsere Herangehensweise ist es, die Ziele durch die Augen eines sechsjährigen Mädchens zu betrachten und zu fragen: »Wie messe ich das? « Die Antwort wird einfach und zu befolgen sein.

- Die richtigen Schritte setzen/Methoden einsetzen

Das ist klar der anspruchsvollste Teil. Aber es ist ja auch das eigentliche Kerngeschäft von Coaches. In der Praxis sind dabei die beiden größten Schwierigkeiten: erstens sofort eingangs zu entscheiden, ob man angesichts dieser Ziele und der Ausgangslage als Coach überhaupt etwas machen darf oder kann - oder ob hier Psychotherapie, Fachberatung oder etwa Organisationsentwicklung gefragt ist. Zweitens gilt es, in der Folge flexibel genug zu sein, wenn nötig von einem eingeschlagenen Weg auch wieder abzubiegen oder von der Fortführung ganz abzulassen.

- Den eingetretenen (Miss-)Erfolg kontrollieren

Bei professionell definierten Zielen sollte dies kein Problem sein, bei qualitativen Zielen erheben wir die Veränderung in Prozent.

\section{Schlussfolgerungen}

Wir als in der Praxis Tätige haben großes Interesse an einer für uns handlungsleitenden Theorie. Wir kritisieren jedoch die gängigen theoretischen Modelle der Evaluation als zu kompliziert und daher als nicht geeignet für das, was Coaches für ihre Kundinnen und Kunden wirklich brauchen. Wir müssen wissen, welche Wirkfaktoren Erfolg bzw. Misserfolg und somit Zielerreichung als Qualität im 
Coaching herbeiführen. Es sollte transparent sein, aus welchen Benchmarkings diese Erkenntnisse gewonnen wurden und mit welcher Wahrscheinlichkeit die Faktoren zum Erfolg eines Coachings beitragen. Und die gewonnenen Erkenntnisse sollten einfach und praktikabel sein. Die Wirkfaktoren zu eruieren, ist unseres Erachtens die Aufgabe von Wissenschaft und Forschung. Dies setzt jedoch voraus, dass Wissenschaft und Forschung zuerst die erfolgreichen bzw. erfolglosen Coachings identifizieren. Das kann nur anhand der Ergebnisse erfolgen. Diese müssen primär evaluiert werden, erst dann kann und soll Ursachenforschung betrieben werden.

Dadurch wäre es möglich, einen "Stand der Technik« im Coaching zu definieren. Dieser wiederum ist notwendig, um zwischen sachgemäßer und unsachgemäßer Leistung zu unterscheiden, was natürlich auch juristische Konsequenzen hat, wie insbesondere Gewährleistungs-, Erfüllungs- und Schadensersatzansprüche.

Hier braucht es dringend eine Umorientierung, und diese wollen wir mit unserem Beitrag anstoßen.

\section{Literatur}

Bischof, A. (2011). Evaluation von Coaching - Stand und notwendige Standardisierung. Coaching-Magazin, 4.

Geißler, H., Wegener, R. (Hrsg.) (2015). Bewertung von Coachingprozessen. Wiesbaden: Springer. Greif, S. (2016). Wie wirksam ist Coaching? Ein umfassendes Evaluationsmodell für Praxis und Forschung. In R. Wegener, M. Loebbert, A. Fritze (Hrsg.), Coaching-Praxisfelder (S. 161-182). Wiesbaden: Springer.

Harramach, N. (1995). Trainings-Erfolgs-Kontrolle. München: Verlag Neuer Merkur.

Heß, T., Roth, W. L. (2001). Professionelles Coaching. Eine Expertenbefragung zur Qualitätseinschätzung und -entwicklung. Heidelberg: Asanger.

Kirkpatrick, D. L. (1977). Evaluating Training Programs: Evidence vs. Proof. Training \& Development Journal, 31 (11), 9-12.

Migge, B. (2013). Schema Coaching. Einführung und Praxis: Grundlagen, Methoden, Fallbeispiele. Weinheim: Beltz.

Runde, B. (2016). Coaching-Evaluation - Kurzfragebogen für die Abschluss-Sitzung. www.coaa ching-tools.de/freie-tools/coaching-evaluation-kurzfragebogen-fuer-die-abschluss-sitzung. html [17.2.2017]. 


\section{Führung entwickeln und Wirkung entfalten}

\section{Zum Zusammenhang von Coaching und Change-Management}

Mathias Hofmann

Anliegen dieses Beitrags ist es, anhand eines realen Projektes in einer deutschen Stadtverwaltung das Zusammenwirken von Coaching und Change-Management zu thematisieren. Das Projekt wird mit Rahmendaten, Zielen und Architektur dargestellt. Dabei werden die zugrunde liegenden methodischen Gedanken erläutert, wie sich in einem Veränderungsprozess mit einer Führungsmannschaft gemeinsam Erfolg erreichen lässt. Die Verlaufsbeschreibung und die Schilderung einzelner Ereignisse veranschaulichen die Wirkung und gegenseitige Beeinflussung von Coaching und Change. Evaluationsinstrumente werden vorgestellt, und die Steuerung des Projektes wird beschrieben.

Der Praxisfall, der in diesem Beitrag beschrieben wird, ist ein Veränderungsprojekt zur Personal- und Organisationsentwicklung der Stadtverwaltung in einer norddeutschen Kreisstadt mit etwa 80000 Einwohnern von 2010 bis 2015. Zentrales Thema war »Führung«. In der Stadtverwaltung sind etwa 1000 Mitarbeiterinnen und Mitarbeiter tätig. Die Führungshierarchie ist sehr flach gestaltet: Der Bürgermeister steht einem (mit ihm) sechsköpfigen Verwaltungsvorstand vor, dessen Mitglieder die einzelnen Geschäftskreise mit Bereichsleitern führen. Unterhalb der 33 Bereichsleiter gibt es in der Regel keine weitere disziplinarische Führungsebene. Das Hauptziel des Projektes war die Qualifizierung der Führung angesichts besonderer Herausforderungen an die Stadtverwaltung.

Für das Projekt waren die folgenden zwei Motivatoren wichtig:

- Der Bürgermeister hatte das Ziel, an der Weiterentwicklung der Führungskompetenz und der Umsetzung des von der Personalentwicklung mit Führungskräften entwickelten Führungsprofils zu arbeiten.

- Die Kommune stand vor großen strategischen Herausforderungen. Gleichzeitig war die Haushaltskonsolidierung zu erreichen, größere Infrastruk- 
turprojekte waren umzusetzen und eine Bildungsplanung mit umfassender Beteiligung zu realisieren.

Im Change-Management galt es also, betriebswirtschaftlich hart definierte Ziele mit einer Qualifizierung der Führungskräfte und einer Optimierung der Zusammenarbeit unter ihnen zu erreichen. Organisationales Lernen (so erreichen wir leichter gemeinsam die Ziele) wurde verbunden mit individuellem Lernen (so führe ich meine Mitarbeiterinnen und Mitarbeiter besser im Sinne der Ziele, vgl. Hofmann, 2010). Der innovative Charakter des Projektes bestand in der Verbindung dieser Aspekte und der Verantwortung der Führungskräfte insgesamt für ihre eigene Qualifizierung: Sie definierten die Ziele und Themen als Gruppe der Führungskräfte gemeinschaftlich und bewerteten die Zielerreichung fortlaufend. Das setzte von Beginn an eine hohe Bereitschaft zur Zusammenarbeit voraus und förderte sie gleichzeitig im weiteren Projektverlauf. Die Ziele für die Haushaltskonsolidierung, die Infrastrukturentwicklung und die Bildungsplanung wurden mit Kennzahlen und Meilensteinen formuliert.

Zwei Zielsysteme wurden so parallel geführt:

- operative Ziele, die mit Kennzahlen und Meilensteinen vorgegeben waren;

- Ziele für Führung und Zusammenarbeit, die partizipativ formuliert und wiederholt bewertet und weiterentwickelt wurden (vgl. Abbildung 1).

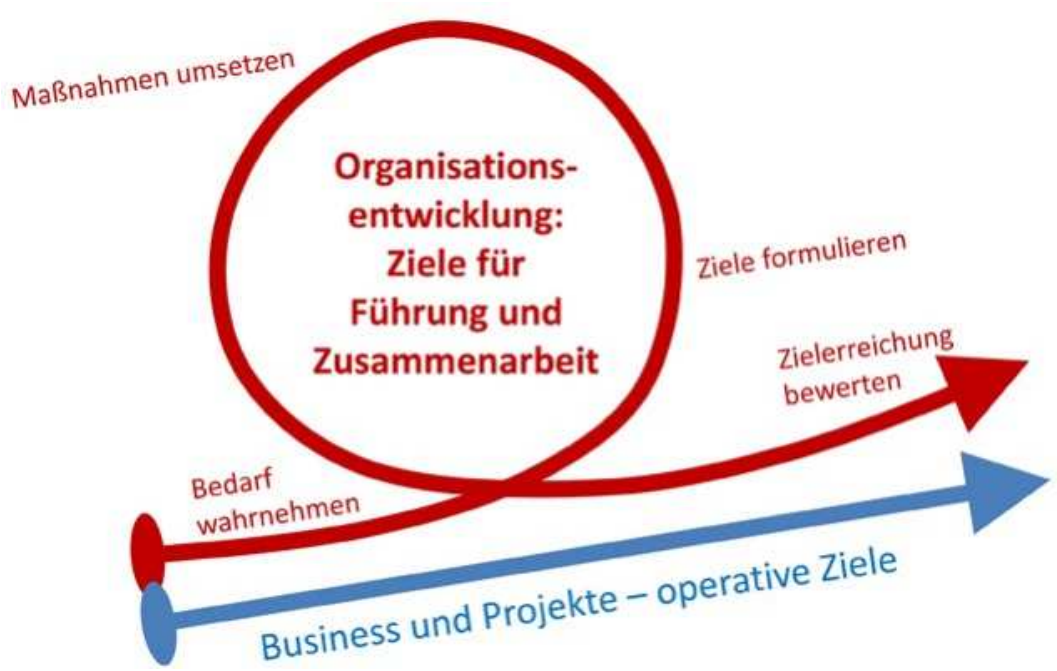

Abbildung 1: Operative Ziele, begleitet von der gemeinsamen Entwicklung und Verfolgung der Führungs- und Zusammenarbeitsziele (vgl. Flick, 2008, S. 19) 

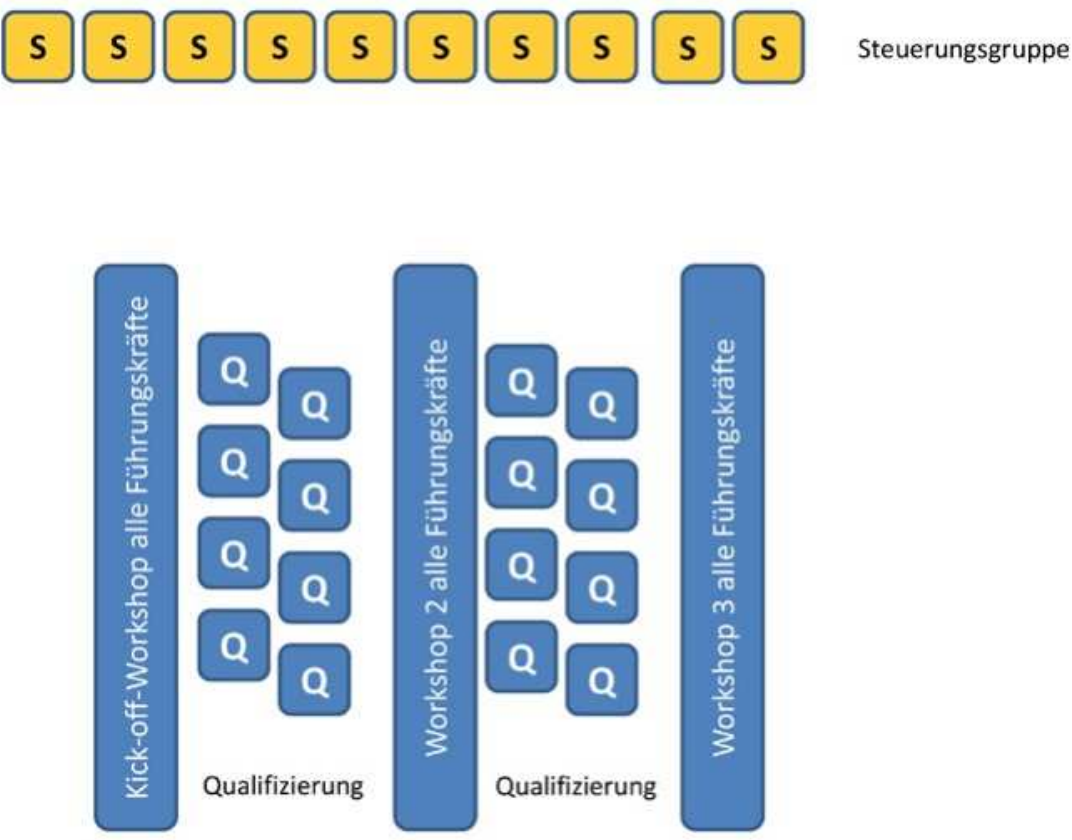

2010

2011

2012

2013

Abbildung 2: Erste Planung des Projektes zum Projektstart

Mensch und System in der Entwicklung komplementär zu verbinden (Strikker, 2007), war sowohl die zentrale Haltung als auch das Ziel des Projektes. Die einzelnen Führungskräfte entwickeln ihre Kompetenzen durch Coaching und Training besser in einem sich wandelnden Umfeld vertrauensvoller und effektiver Zusammenarbeit der Führungskräfte. Das System der Führungskräfte entwickelt sich besser, indem sich alle Beteiligten reflektiert und kompetent einbringen.

Die erste Planung (vgl. Abbildung 2) und die fortlaufende Steuerung des Projektes verantwortete eine Steuerungsgruppe (Hofmann \& Strikker, 2007) aus Bürgermeister, Personalentwicklerin und der Projektleiterin des externen Beratungsunternehmens. Die Projektleiterin hatte hier die Funktion einer Fachberaterin zur Gestaltung des Change-Projektes und verantwortete gleichzeitig die Umsetzung von Workshops, Trainings und Coachings (die sie selbst oder ihre Berater-Kollegen durchführten). Entscheidungen der Steuerungsgruppe wurden gemeinsam gefällt und vertreten.

Das Projekt war zunächst auf zwei Jahre angelegt. Es bestand von Beginn an die Bereitschaft, Elemente von Coaching einzelner Führungskräfte, Teament- 
wicklung/Gruppencoaching, Trainings, Lerntandems/kollegiale Beratung und Workshops in einer Gesamtarchitektur zu verbinden. Diese Architektur wurde bewusst offen angelegt, die erste Planung wurde durch die aktive Steuerung im Verlauf regelmäßig überprüft und im agilen Projektmanagement den Bedarfen angepasst. Ein zentrales Instrument zum Controlling war das regelmäßige Feedback der Führungskräfte zu Wirkung und Zielerreichung.

Zunächst wurde lediglich ein Kick-off-Workshop für alle Führungskräfte geplant, dazu zwei Phasen von Führungskräftequalifizierung über jeweils ein Jahr, die je mit einem Workshop zur gemeinsamen Auswertung abgeschlossen wurden (Abbildung 2). Coaching wurde den Führungskräften optional ergänzend angeboten.

\section{Wirkung und Erfolg planen}

Im Projekt wurde auf eine externe wissenschaftliche Evaluation verzichtet. Gleichwohl sollten die einzelnen Maßnahmen wie auch das Gesamtprojekt nachweislich erfolgreich sein. Die Steuerungsgruppe konzipierte daher eine stetige interne Evaluation durch die Beteiligten im Sinne von "Handlungsforschung", "action research " nach Kromrey: "Ihr Ablauf ist iterativ schleifenartig, ist ein fortwährendes Fragestellen, Antworten, Bewerten, Informieren und Aushandeln« (Kromrey, 2001, S. 129). In der Praxis war es ein fortlaufend initiierter Diskurs über Führung, Führungshandeln und den Projektfortschritt in den Workshops und Qualifizierungen. Die Führungskräfte selbst wurden immer wieder mit der Frage konfrontiert, welche Wirkung sie feststellten. Wiederkehrende Fragen waren: Wie kommen die Projekte voran? Wie effektiv ist die Führung? Wie ist die Zusammenarbeit zwischen den Führungskräften? Wie ist die Außenwirkung in Richtung Bürger und Politik?

Das Projekt folgte der These Festingers (1954, zit. nach Greif, 2015, S. 50), der die komplexe Bewertung als ein Ergebnis sozialer Konstruktion sieht, beziehungsweise der von Brühl (2004, S. 66), für den »Erfolg [...] eine Größe [ist], die von den subjektiven Bewertungen im Unternehmen geprägt ist «. Im Kick-off-Workshop waren die Führungskräfte aufgefordert, ihre subjektiven Bewertungen weitestgehend einzubringen und gemeinsam ein Bild der aktuellen Situation und der Ziele zu formulieren und die Handlungsschritte dorthin zu entscheiden. Change-Management wurde so im Sinne Siegfried Greifs verstanden als "Prozess der kontinuierlichen Exploration, Analyse und Evaluation und des Managements [...] [von] Problemen und Erfolgsrisiken sowie der Erfolgsfaktoren und -chancen [...]. Ziel ist ein hoher Zielerreichungsgrad 
und eine positive Evaluation der Maßnahmen, Ergebnisse und Folgen durch die relevanten Schlüsselpersonen und -gruppen« (Greif, Runde \& Seeberg, 2004, S. 54).

In der Praxis des konkreten Projektes wurden die Führungskräfte im Kickoff-Workshop über das Gesamtprojekt informiert. Sie teilten sich nach einer Einführungsdiskussion in fachlich gemischte Gruppen auf, die sich dann mit den einzelnen operativen Handlungsfeldern (entsprechend den formulierten operativen Zielen) beschäftigten. Sie diskutierten die Herausforderung, die sich für die Führung im Zusammenhang mit dem angestrebten Ziel stellte, und beschrieben den Ist-Stand (Stärken/Schwächen). Als Maßnahmen zur Unterstützung wählten sie aus einem Portfolio geeignete Seminare für sich selbst aus, um die Herausforderungen besser zu meistern (vgl. Abbildung 3).

Die Steuerungsgruppe vertraute darauf, dass bei einer Gruppengröße von etwa vierzig Personen ein hoher Grad an wahrhaftigen Rückmeldungen entsteht und sich »blinde Flecken« ausgleichen.

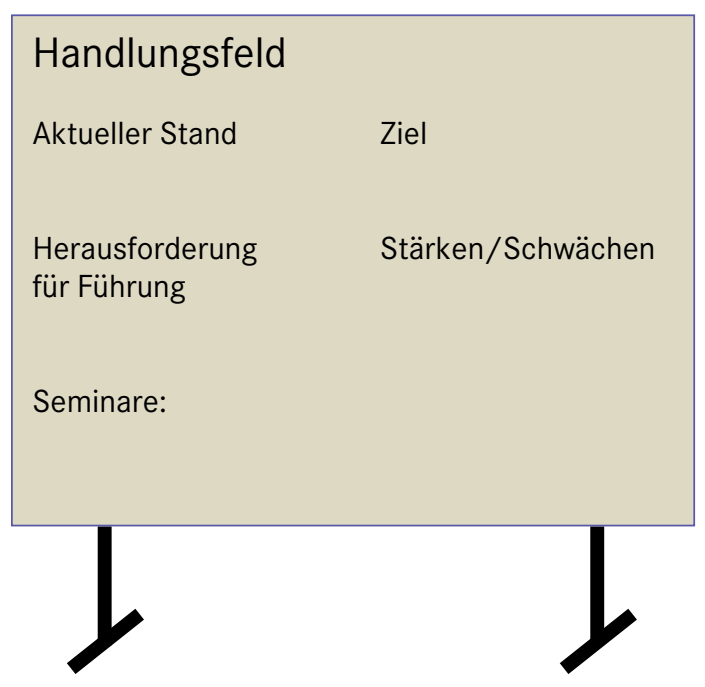

Seminaroptionen:
Selbstmanagement
Verantwortlich führen
Problemlösung
Strategisch planen
Repräsentation
Unternehmerisch handeln
Projektverhandlung
Strukturiert organisieren
Produktivität steigern
Moderation
Teamführung
Delegation
Konfliktlösung
Gesundes Führen

Abbildung 3: Instrument Planung bottom-up, Pinnwand der Gruppenarbeit eines operativen Handlungsfeldes zur Diskussion von Führung und zur Auswahl von unterstützenden Seminarthemen 


\section{Umsetzung und Anpassung der Planung}

Die danach ausgewählten spezifischen Seminare wurden fallorientiert auf die Bedarfe des Handlungsfeldes konzipiert. Die Führungskräfte, die gemeinsam in den Handlungsfeldern tätig waren, arbeiteten als Gruppe in Seminaren. Diese enthielten regelmäßig Coaching-Elemente. Jedes Seminar begann mit den Fragestellungen der Teilnehmerinnen und Teilnehmer aus der Praxis, die es zu bearbeiten galt. Der Input der Trainer/Moderatorinnen war auf Anwendbarkeit für diese Fragen ausgerichtet. Tatsächliche Situationen und Fragestellungen aus den operativen Projekten wurden mit den vermittelten Methoden bearbeitet. Die Führungskräfte, die diese Fragen und Fälle einbrachten, gewannen konkrete Erkenntnisse und neue Ideen oder Vorhaben für ihr Führungshandeln und ihr Management. Lösungsorientierte Fragen und Feedback durch die Kolleginnen und Kollegen wurden ständig zur Reflexion und weiteren Entscheidungsfindung genutzt. Um einen hohen Praxisnutzen für alle Teilnehmerinnen und Teilnehmer zu erreichen, wurden mehrere Fälle parallel bearbeitet, anschließend wurde der Nutzen der Methodik gemeinsam reflektiert. Der Transfer wurde über formulierte Transfervorhaben und die gegenseitige Beratung in Lerntandems unterstützt.

Ein fach- und hierarchieübergreifender Mix der Führungskräfte in den einzelnen Seminaren verbesserte laut Feedback der Führungskräfte das Verständnis zwischen den unterschiedlichen Interessen und Verantwortlichkeiten der Fachbereiche und Führungsebenen und vereinfachte die alltägliche Zusammenarbeit. Durch die vielfach explizite Thematisierung von Führung und Zusammenarbeit in, während, neben und nach den Qualifizierungen wurden auch im alltäglichen Miteinander Meinungen zu Führung ausgetauscht, die Zusammenarbeit wurde vermehrt explizit besprochen und entwickelte sich positiv. Fachliche Konflikte wurden als solche besser angesprochen, die Sichtweise des anderen Fachbereiches eher akzeptiert und gemeinsame Lösungen auf der Arbeitsebene erarbeitet.

Ein weiteres Ergebnis der Evaluation im Führungskräfte-Workshop war, dass ein einmaliger Impuls in einer Qualifizierung nicht ausreiche. In der Praxis entstünden fortlaufend Fragestellungen, für die eine kontinuierliche Begleitung durch Einzelcoaching wichtig sei.

Zum anderen wurden als Hinderungsgrund für eine bessere Zusammenarbeit zwischen einzelnen Fachbereichen von mehreren Führungskräften die Differenzen im Verwaltungsvorstand genannt. Dieser zweite Grund wurde vielfach in informellen Gesprächen erwähnt und beruhte bei Nachfragen auf Hörensagen und den Empfindungen der Führungskräfte, die in den Verwaltungsvorstandssitzungen ja nicht teilnahmen. 
In der Auswertung der Steuerungsgruppe wurde so deutlich, dass die $\mathrm{Zu}$ sammenarbeit im Verwaltungsvorstand eine zentrale Bedeutung für den Erfolg des Projektes hatte. Als Maßnahmen entschied die Steuerungsgruppe (auf Vorschlag des Bürgermeisters) ein Einzelcoaching des Bürgermeisters zur Führung des Verwaltungsvorstandes und ein Teamcoaching des Verwaltungsvorstandes zur Zusammenarbeit und Außenwirkung. Entscheidender Grundgedanke war ein einheitliches Vorleben eines Vorbildes als Top-Führungskräfte.

Außerdem wurde das Angebot an Coaching für einzelne Führungskräfte intensiviert, um durch kontinuierliche Begleitung mit Einzelcoachings den Transfer aus den Trainings zu verbessern.

Bereits vor dem Führungskräfteworkshop wurde die Erweiterung des Projektes um das Führungsfeedback geplant. Die neue Architektur sah demnach wie folgt aus (vgl. Abbildung 4. $\mathrm{S}=$ Steuerungsgruppe, $\mathrm{BM}=$ Bürgermeister, $\mathrm{VV}=$ Verwaltungsvorstand, $\mathrm{Q}=$ Qualifizierung, FFB = Führungsfeedback, FK = Führungskräfte):

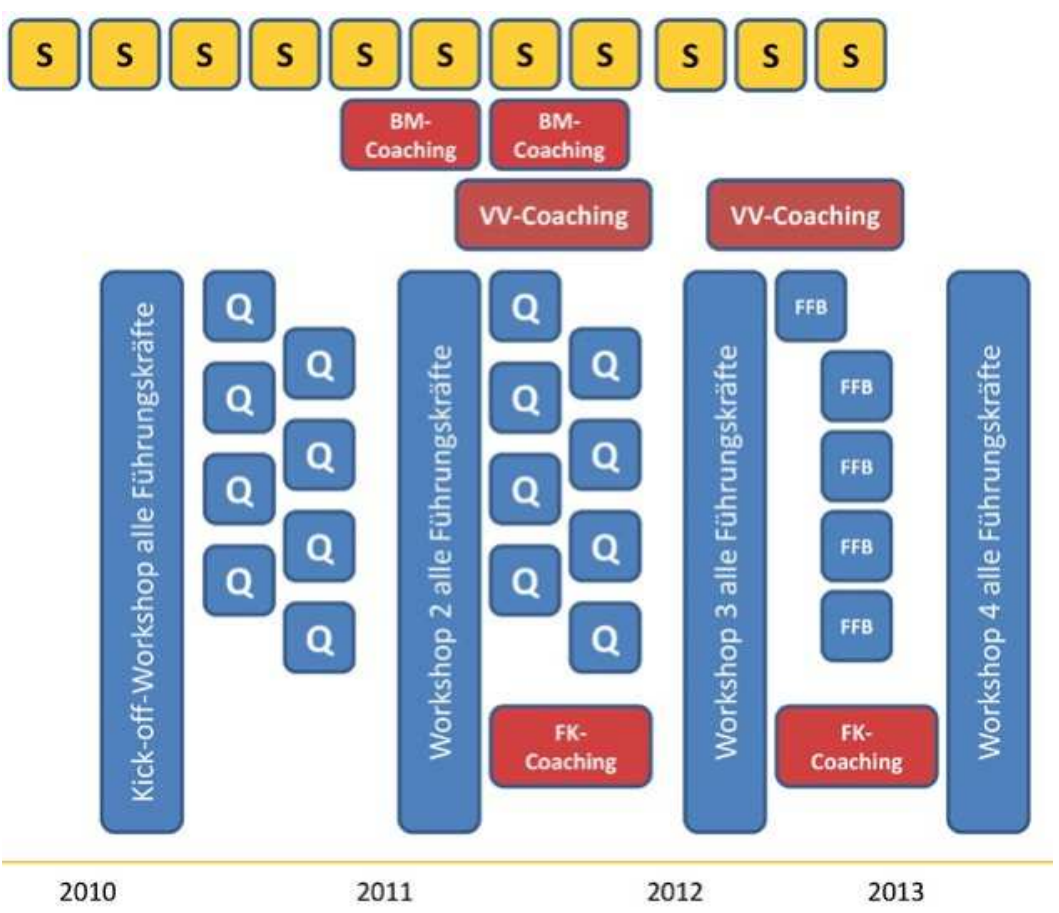

Abbildung 4: Angepasste Projektplanung vor Workshop 2 
In Workshop 2 werteten die Führungskräfte gemeinsam die bisherigen Qualifizierungen danach aus, was für sie im Führungshandeln hilfreich war, welche Tools, Modelle und Methoden an welcher Stelle sie wirksam eingesetzt hatten. Sie entwickelten Empfehlungen, welche Methoden und Seminare auch für andere Führungskräfte zur Verfügung gestellt werden sollten. Entsprechend dieser Evaluation durch die Zielgruppe wurde von der Personalentwicklung die zweite Staffel an Qualifizierungen wiederum spezifisch für einzelne Führungskräfte gestaltet. Wie im Kick-off-Workshop wurde nach der Bewertung der Zielerreichung der Vorstaffel der neue Bedarf wahrgenommen, es wurden neue Ziele formuliert und Maßnahmen beschlossen. Die Umsetzung folgte (vgl. Abbildung 1 - eine neue Schleife).

Workshop 2 wurde außerdem genutzt, um den Stand der Zielerreichung in den operativen Handlungsfeldern zu besprechen und die weitere Planung zu justieren.

\section{Coaching und Change-Management}

Coaching war in der Organisation durch die Personalentwicklung seit mehreren Jahren eingeführt. Die Aussage des Bürgermeisters in Workshop 2, er lasse sich coachen, erhöhte die Akzeptanz von Coaching unter den Führungskräften. Dazu trug ebenso die positive Rezeption der Coaching- und Fallberatungs-Elemente in den Qualifizierungen bei. Die Zielsetzung der Führungskräftecoachings entsprach der Zielsetzung des Gesamtprojektes: Verbesserung der Führungskompetenz und handlungsorientierte Lösung spezifischer Fragen und Fälle. Die Coachings wurden jeweils mit der Personalentwicklerin und der Führungskraft des Coachee abgestimmt (Klein \& Strikker, 2003).

Der Bürgermeister thematisierte im Coaching die eigene Führung und die Zusammenarbeit mit den von ihm geführten Personen. In mehreren Coachings entwickelte er eine reflektierte Konzeption seiner Führung der eigenen Person, des Verwaltungsvorstandes und der Gesamtorganisation über den aktuellen Zeitpunkt hinaus.

Das Teamcoaching des Verwaltungsvorstandes bearbeitete die Art der operativen Zusammenarbeit und die gemeinsame Wirkung nach außen. Das System Verwaltungsvorstand, also das "Wir", stand im Mittelpunkt, das gemeinsame Ziel, die gemeinsamen Regeln, die gemeinsame Kommunikation, das Eintreten Einzelner für das Gemeinsame. Auch zukünftige Risiken und Personalwechsel wurden thematisiert.

Die Wirkung des Verwaltungsvorstands-Coaching auf das Gesamtprojekt war sehr deutlich wahrnehmbar. Beeindruckend zeigte dies ein gemeinsamer Auf- 
tritt der Verwaltungsvorstandsmitglieder in Workshop 3 mit der gemeinsamen Vorstellung zum Thema »Führungsfeedback «. Bis zu diesem Zeitpunkt hatte der Bürgermeister alle Präsentationen zu Führung alleine gestaltet. Der gemeinsame Auftritt ließ ein neues Bild entstehen und brachte positive Kommentare durch die Führungskräfte bereits in den Workshop-Pausen. Im weiteren Verlauf des Projekts wurde die bereichsübergreifende fachliche Zusammenarbeit der Führungskräfte zunehmend besser zurückgemeldet - obschon im Verwaltungsvorstand weiterhin divergierende Vorstellungen sachlich ausgehandelt wurden. Der entscheidende Unterschied war, dass die Mitglieder des Verwaltungsvorstandes ihre Kommunikation besser abstimmten und ein gemeinsames Auftreten zeigten.

Die Coachings waren durch den Verlauf und die Steuerung des Change-Projektes initiiert worden. Wie fast immer lassen sich im Change-Management Ergebnis und Verlauf nicht hundertprozentig vorausplanen. Dazu bedarf es eben der Steuerung, und Veränderungsprojekte verändern sich dann kontrolliert während der Umsetzung.

Coaching ist für Change-Projekte eine häufig hilfreiche Intervention. Organisatorische Veränderungen erfordern persönliche Entwicklungen. Für diese individuellen Entwicklungen von Führungskräften ist Coaching ein geeignetes Instrument. Einzelne Führungskräfte werden spezifisch unterstützt, damit sie die Anforderungen der organisationalen Veränderung erfüllen und mit ihrem Führungshandeln in ihren Verantwortungsbereich hineinwirken können. Sie werden durch Coaching in die Lage versetzt, ihren Beitrag zum Change zu leisten.

Nicht jedes Change-Projekt bedarf begleitender Coaching-Maßnahmen. Falls aber in einem Change-Vorhaben der Bedarf nach individuellem Coaching erkennbar wird, sollte im Sinne des komplementären Ansatzes beides zusammen konzipiert werden, sodass Ziele kongruent sind und Ergebnisse zusammenwirken können. Eine gemeinsame Architektur und eine Gesamtsteuerung von Coaching im Change bildet dies ab.

\section{Wirkung und Erfolge wahrnehmen}

Die partizipative Evaluation von Führung und Zusammenarbeit durch die Beteiligten wurde im Projekt wie geplant iterativ-schleifenartig fortgesetzt. Wirkungen und Veränderungen, insbesondere durch die Qualifizierung, die Workshops, die Coachings und die erste Umsetzung des Führungsfeedbacks, wurden in Workshop 4 bei allen Führungskräften explizit nachgefragt.

Dabei erkundigte sich die Moderation (im Auftrag der Steuerungsgruppe) nicht nur danach, was jede einzelne Führungskraft bei sich selbst an Veränderung 
wahrnahm, sondern erweiterte die Fragestellung (systemisch) auf die Wahrnehmung von Veränderungen bei anderen Kolleginnen und Kollegen, den Mitarbeitenden und den Bürgern und Bürgerinnen bzw. der Politik als "Kunden«.

Als Methode wurde die »stumme Diskussion« gewählt, bei der Statements auf Pinnwände geschrieben werden und sich Dialoge so automatisch dokumentieren (Schäffer, 2009; vgl. Abbildung 5).

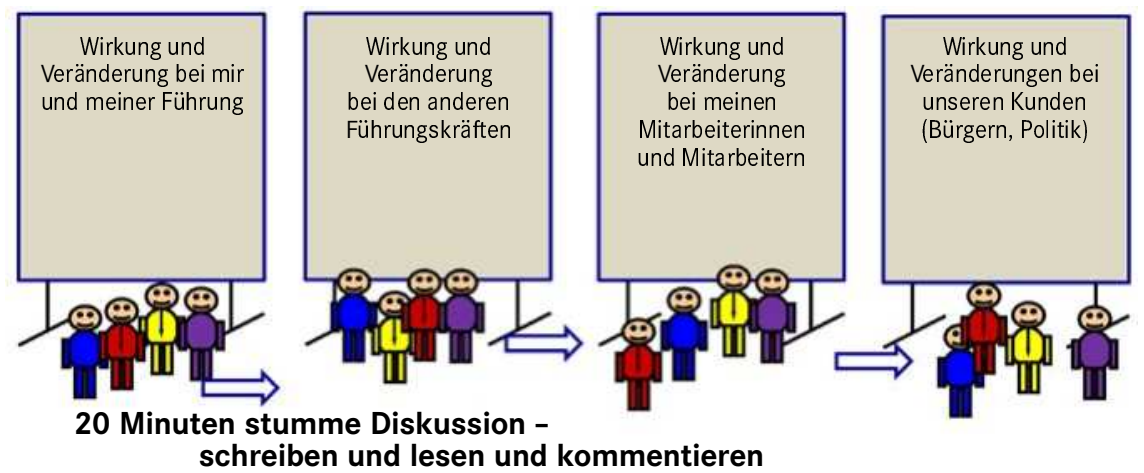

Abbildung 5: Instrument »gemeinsame Bewertung«, Aufgabe an alle Führungskräfte zur Bewertung des Gesamtprojektes nach drei Jahren (vgl. Hofmann \& Strikker, 2009)

Das Ergebnis war außerordentlich positiv, auch wenn sicher konstatiert werden muss, dass eine Bewertung von Beteiligten (und damit subjektiv Betroffenen) tendenziell emotionale Elemente stärkt und eher eindeutig ausfällt (positiv oder negativ). Abbildung 6 zeigt beispielhaft einige Niederschriften auf die Frage: »Was merken die Kunden und Bürger von der Veränderung?»

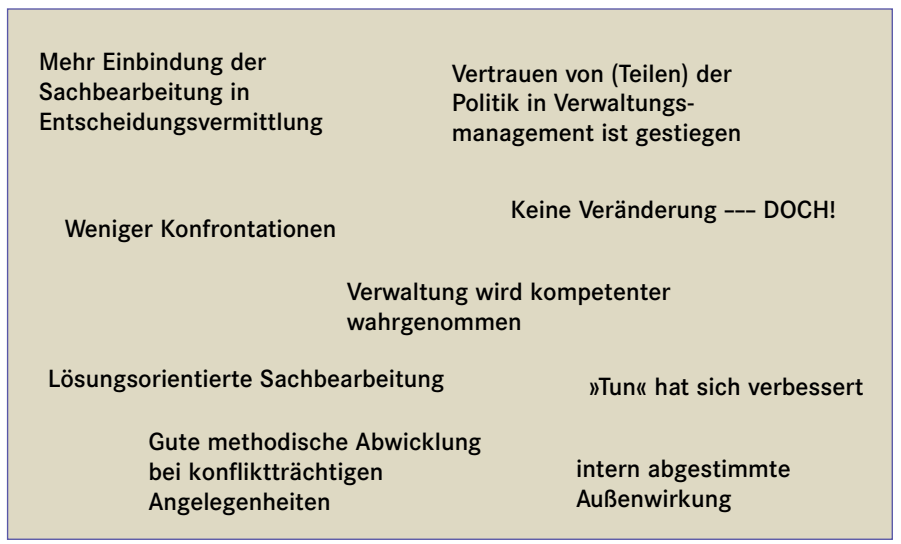

Abbildung 6: Schriftliche Rückmeldungen auf einer Pinnwand nach der »stummen Diskussion« 
Der Erfolg des Change-Projektes zeigt sich auch in der zufriedenstellenden Zielerreichung mit Blick auf die operativen Ziele: Das sehr ambitionierte Ziel der Haushaltskonsolidierung wurde erreicht, die Bildungsplanung war entsprechend den Vorgaben fortgeschritten. Lediglich die Infrastrukturentwicklung war in Teilen nicht im Zeitplan, begründet durch externe Faktoren.

\section{Fazit}

Aufgrund dieser Fallstudie scheint relevant zu sein, wie Coaching in einem Change-Projekt konkret eingebettet wird, um eine gegenseitige positive Beeinflussung zu erreichen. Die Verbindung von Coaching und Change-Management in einem System mit gemeinsamen Zielen und gewollt gegenseitiger Beeinflussung erzeugt eine komplementäre und kongruente Vorgehensweise. Ein Coaching, mit dem eine Führungskraft unterstützt wird, dem Ziel des Veränderungsvorhabens entgegenlaufende persönliche Ziele zu erreichen, ist so undenkbar. Für die weitere Forschung wäre es interessant, verschiedene Modelle dieses komplementären Ansatzes von Coaching und Change zu beschreiben und hinsichtlich ihrer Wirkung zu vergleichen, soweit dies angesichts verschiedener Organisationskulturen möglich ist.

Die Verbindung der harten operativen Ziele mit den Entwicklungszielen der einzelnen Führungskräfte und hinsichtlich deren Zusammenarbeit zeigt sich in dem geschilderten Projekt ebenfalls als Erfolgsfaktor. Die operativen Ziele vermitteln Sinn für die Qualifizierung von Führung und bieten beste Möglichkeiten zur Erfolgskontrolle. Ein Ergebnis des Projektes war eine umfangreiche Toolbox zum Führungshandeln in der Kommune. Der wichtigste Baustein daraus war die Reflexionskompetenz und das gegenseitige Feedback in vertrauensvoller Kollegialität. Im vorliegenden Fall wurde dieses Instrumentarium auf Basis des Führungsleitbildes in Zusammenarbeit der Führungskräfte mit der Personalentwicklung und den externen Trainern im laufenden Projekt entwickelt. Eine interessante Frage ist die weitere Entwicklung dieses Instrumentariums in einem ständigen Prozess der Führungskräfte der Organisation bei sich verändernden Herausforderungen und neuen operativen Zielen.

Es stellt sich ferner die Frage, inwieweit die Selbstevaluation der Beteiligten sich weiter professionalisieren lässt. Der Nutzen für das Change-Management ist im beschriebenen Fall sehr deutlich: Die Motivation der Beteiligten wird durch diese Form der Partizipation angesprochen, die Führungskräfte haben die Veränderung aktiv gestaltet. Außerdem konnte durch das unmittelbare Gespräch miteinander und durch den Diskurs vor Ort Einvernehmen hergestellt werden. 
Das ist die beste Basis für schnelle Reaktionen und Entscheidungen der Steuerung, die wiederum von allen unisono vertreten werden.

Fallbeispiele und Praxisberichte liefern ohne wissenschaftliche Fundierung zunächst nur spezifische subjektive Erkenntnisse und keine allgemeingültigen Ergebnisse. Die Wirkfaktoren sind vielfältig, ihre Bewertung verlangt nach einer Distanz, welche die Beteiligten kaum aufbringen können. Gleichzeitig liefert die große Nähe zum Projekt viele Eindrücke, die mit einer angemessenen Reflexion und Auswertung außerordentlich hilfreich sind für die Steuerung. Bei dieser Form der Selbstevaluation durch die Beteiligten mit dem Anspruch, die Erkenntnisse aus Befragungen und geplantem Feedback direkt für die Steuerung zu nutzen (im Sinne von action research wie oben beschrieben), müssen die Akteure selbst für eine gewisse Distanz sorgen. Im beschriebenen Projekt wurde dies gelöst, indem durchgehend zwei Personen auf Beraterseite stark involviert waren und sich zu diesem Projekt untereinander und auch mit anderen nicht beteiligten Kolleginnen und Kollegen der Beratungsgesellschaft kollegial und vertraulich berieten. Die durchgehende Steuerung mit den drei Repräsentanten Gesamtführung, interne Personalentwicklung und externe Prozessexpertise führte zu einer konstruktiven Diskussion aus verschiedenen Blickrichtungen. Durch die vereinbarte klare Rollenaufteilung, verbunden mit dem hohen Vertrauen zwischen den Personen, konnten alle drei ihre jeweiligen Stärken einbringen. Durch die Trennung des operativen Managements im Verwaltungsvorstand vom Change-Management in der Steuerungsgruppe konnte die Projektsteuerung unabhängig vom alltäglichen Geschäft bis hin zur Planung eines Vorstandscoachings erfolgen.

\section{Literatur}

Brühl, R. (2004). Controlling. Grundlagen des Erfolgscontrollings. München: Vahlen.

Flick, U. (2008). Qualitative Evaluationsforschung. Konzepte, Methoden, Umsetzungen. Reinbek b. Hamburg: Rowohlt.

Greif, S. (2015). Evaluation von Coaching: Eine schwer zu bewertende Dienstleistung. In A. Schreyögg, C. Schmidt-Lellek (Hrsg.), Die Professionalisierung von Coaching (S. 47-69). Wiesbaden: Springer.

Greif, S., Runde, B., Seeberg, I. (2004). Erfolge und Misserfolge beim Change Management. Göttingen: Hogrefe.

Hofmann, M. (2010). Durch Lernen verändern, durch Veränderungen lernen. In J. Graf (Hrsg.), Manager Seminare 2010, Das Jahrbuch der Management-Weiterbildung (S. 87-102). Bonn: managerSeminare.

Hofmann, M., Strikker, F. (2009). Steuerungsgruppe. In A. Leão, M. Hofmann (Hrsg.), Fit for Change. 44 praxisbewährte Tools und Methoden im Change für Trainer, Moderatoren, Coaches und Change Manager (S. 62-67). Bonn: managerSeminare. 
Hofmann, M., Strikker, F., Timmermann, D. (2014). Return On Investment im Change Management. Studienbrief im Masterstudiengang Business Coaching und Change-Management. Hamburg: Euro FH.

Klein, S., Strikker, H. (2003). Auftragscoaching oder: Die Tücken der Dreiecksbeziehung. In J. Graf (Hrsg.), Manager Seminare 2003. Das Jahrbuch der Management-Weiterbildung. Bonn: managerSeminare.

Kromrey, H. (2001). Evaluation, ein vielschichtiges Konzept. Begriff und Methodik von Evaluierung und Evaluationsforschung. Empfehlungen für die Praxis. Sozialwissenschaften und Berufspraxis (SUB), 24 (2), 105-131.

Schäffer, T. (2009). Stumme Diskussion. In A. Leão, M. Hofmann (Hrsg.), Fit for Change II. 40 praxisbewährte Tools und Methoden im Change für Trainer, Moderatoren, Coaches und Change Manager (S. 224-230). Bonn: managerSeminare.

Strikker, H. (2007). Komplementär-Coaching. Mensch und System komplementär verbinden. Paderborn: Jungfermann. 


\title{
Coaching-Kultur in deutschsprachigen Organisationen
}

\author{
Das Konzept der resilienten und agilen Organisation
}

Axel Klimek und Werner Stork

In unserer globalisierten und digitalisierten Welt sind neue organisatorische Konzepte und Lösungen gefragt, um Unternehmen für aktuelle und künftige Herausforderungen besser zu wappnen. Der Beitrag beschreibt Hintergründe und Ursachen dieser Entwicklungen; er erläutert den neuen Ansatz der resilienten und agilen Organisation und die besondere Bedeutung, die dabei der Coaching-Kultur zukommt. Dann werden die Erkenntnisse einer Studie zur "Coaching-Kultur in deutschsprachigen Organisation« dargelegt. Der Beitrag schließt mit Anregungen für weiterführende Konzeptentwicklungen und Studien.

Die globalisierte und zunehmend digitalisierte Wirtschaft zeichnet sich durch eine enorme Komplexitätserhöhung und gesteigerte Geschwindigkeiten auf allen Ebenen aus. Gleichzeitig werden die Menschen - und auch die Organisationen, in denen sie arbeiten - kognitiv und mental immer stärker gefordert. Um als Organisationssystem in diesem Umfeld einen nachhaltigen Mehrwert liefern zu können - oder auch einfach, um überlebensfähig zu bleiben -, müssen Organisationen verstärkt als System resilient sein und agil agieren können.

Ein wichtiger Hebel für die Resilienz und Agilität von Organisationen liegt in der Qualität der direkten Zusammenarbeit und Kommunikation an den unzähligen Schnittstellen innerhalb der Organisation und an den Kontaktstellen zu unterschiedlichen Stakeholdern. An diesem Punkt setzt das Konzept der Coaching-Kultur an. Wir verstehen Coaching-Kultur auf organisatorischer Ebene als qualitative Dimension von Unternehmen, die sie befähigt, mit externen und internen Veränderungen (auch mit Krisen) resilienter und agiler umzugehen. Coaching-Kultur ist damit in unserem Verständnis eine Qualität von unternehmerischer Wettbewerbsfähigkeit sowie »Fitness und Gesundheit«.

An der Hochschule Darmstadt besteht seit 2013 das Forschungs- und Projektprogramm RODA (Resiliente Organisation der Digitalen Arbeit). Bei RODA geht 
es darum, das Konzept der Resilienz systematisch für die Organisation und das Management von Unternehmen nutzbar zu machen. Resilienz auf Unternehmensebene kann als systemische Widerstandsfähigkeit und Agilität verstanden werden.

Im RODA-Programm werden die Handlungsebenen des Individuums, des Teams, der Führung und der Organisation untersucht. Auf organisatorischer Ebene spielt dabei das Konzept der Coaching-Kultur eine zentrale Rolle.

\section{Neue Modelle und Konzepte zur Organisation der Arbeit werden gesucht}

\section{Der Ansatz der Resilienten Organisation der Digitalen Arbeit (RODA)}

Aus den Ergebnissen einer explorativen Auswertung (Metastudie) von 171 einzelnen Studien aus Wissenschaft, Unternehmenspraxis und Öffentlichkeit/ Presse, die das Institut für Arbeitsdesign und Zukunftstechnologien e. V. aus München durchgeführt hat (Birk, Nelsen, Lackerbauer \& Kirchner, 2015), ergibt sich, dass faktisch keine fundierte kritische Stimme die aktuell massiven Änderungen in der Arbeitswelt anzweifelt: »[...] niemand geht davon aus, dass die aktuellen technischen und gesellschaftlichen Veränderungen keine oder nur marginale Auswirkungen auf die Arbeitswelt haben werden« (ebd., S. 4).

Die neu sich herausbildende Arbeitswelt führt auch zu einem Wandel in der Art und Weise, wie die "neue Arbeit« in den Unternehmen organisiert wird. Tatsächlich ist Arbeit aufgrund der neuen technischen Möglichkeiten in der digitalisierten Arbeitswelt heute deutlich dezentraler und kleinteiliger organisierbar; häufig kann so viel schneller und effizienter gearbeitet werden als in den klassischen Welten der betrieblichen Aufbau- und Ablauforganisation. Gleichzeitig wächst auch die Bedeutung von Teamarbeit, von Zusammenarbeit und Vernetzung, da deutlich mehr Ressourcen und Kompetenzen für die betrieblichen Aufgaben und Ziele gebündelt werden können.

Für Unternehmen stellt sich die Frage, wie für anstehende Aufgaben, Tätigkeiten und Projekte die jeweils geeignete Form der Organisation aussieht. In diesem Bereich wird derzeit viel experimentiert. So werden beispielsweise zunehmend agile und virtuelle Formen der Organisation genutzt, und es entwickeln sich die vielfältigsten neuen Formen der Zusammenarbeit: alle möglichen Ausformungen von Netzwerken, Crowd Sourcing, neue Formen des Outsourcing und neue Konzepte in der Projektarbeit usw. Es ist deshalb auch die Rede von der Notwendigkeit eines neuen Management- und Organisationsverständnisses, es fallen Schlagwörter wie »Schwarmorganisation «, »agiles Management« oder auch »Demokratisierung des Managements«. 
Gleichzeitig ist zu beobachten, dass die kognitiven und mentalen Ressourcen der Beschäftigten bei der Arbeit immer mehr beansprucht werden. Fachleute sprechen insbesondere in Bezug auf die Belastungen und Beanspruchungen im psychisch-mentalen Bereich von regelrechten Ressourcendefiziten. Dass die psychischen und mentalen Ressourcen immer häufiger nicht ausreichen, belegt die stetige und deutliche Zunahme bestimmter psychischer Erkrankungen (Burnout, Depression usw.) und die häufigeren Fälle von Resignation und Rückzug gegenüber Veränderungen im Betrieb. Stressbedingte Erkrankungen nehmen $\mathrm{zu}$, die dadurch bedingten Kosten sind enorm. Ganz offensichtlich verfügen Menschen und Organisationen in den klassischen Organisations- und Arbeitskonzepten nicht über ausreichende psychisch-mentale Ressourcen, um den Anforderungen des modernen Arbeitslebens gerecht zu werden (Lohmann-Haislah, 2012; Stork, 2014; Rose, Müller, Burr, Schulz \& Freude, 2016).

In der neuen Arbeitswelt werden die kognitiven und mentalen Ressourcen sowohl der Menschen als auch der Organisationen für den Unternehmenserfolg immer wichtiger. Dazu gehört ein professioneller Umgang mit allen denkbaren Informationen und Kommunikationsformen und den damit verbundenen subjektiven Perspektiven, Meinungen und Emotionen auf individueller wie auf organisatorischer Ebene. Faktoren wie Stressresilienz, Reagibilität, Innovationsfähigkeit, Konflikt- und Kommunikationsbereitschaft spielen für den wirtschaftlichen Erfolg eine immer entscheidendere Rolle.

Das Forschungs- und Projektprogramm RODA an der Hochschule Darmstadt fokussiert systematisch auf neue organisatorische Modelle der Arbeitsorganisation, die sich nachhaltig positiv auf die psychische Resilienz und Agilität von Mitarbeitenden und gesamten Organisationen auswirken. Das RODA-Programm bildet einen der zentralen Forschungs- und Projektbereiche des Zentrums für nachhaltige Wirtschafts- und Unternehmenspolitik, das Anfang 2017 an der Hochschule Darmstadt gegründet wurde.

Ausgehend von den drei Dimensionen der Resilienz - wirksamer Umgang mit Stressoren, Pflege und Entwicklung der Ressourcen auf Basis von Antreibern und Motiven sowie Ausgleich von Anspannung und Entspannung -, wird im Rahmen des RODA-Programms systematisch versucht, diese Faktoren für die Organisation und das Management nutzbar zu machen. Resilienz auf Unternehmensebene kann als systemische Widerstandsfähigkeit und Agilität verstanden werden. Untersucht werden im RODA-Programm die Handlungsebenen Individuum, Team, Führung und Organisation.

Resiliente Organisationen kann man sich als hybride Organisationen vorstellen, die systematisch Elemente der Hierarchie mit Elementen von Netzwerken verbinden. Diese Besonderheit führt dazu, dass die Organisationen ihre Aktivi- 
täten je nach Anforderungen gezielt unterschiedlich »regeln «. Damit heißt es in solchen Organisationen regelmäßig: »Das kann man auf diese Art und Weise machen oder auch auf jene Art und Weise. « Es gilt damit ein "Sowohl-als-auch" im Gegensatz zu der geläufigen klaren und eindeutigen gemeinsamen strategischen Ausrichtung und Linie, an der alle festhalten (sollten). Wenn es in einer solch hybriden Organisation bei einer Sache (Produkt, Entwicklung, Projekt usw.) vor allem um Schnelligkeit, Effizienz, Genauigkeit und Kosteneinsparungen geht, organisiert man sich eher hierarchisch. Geht es primär um Innovationen, Kreativität, Flexibilität, Verständigung und vertrauensvolle Beziehungen usw., organisiert man sich eher als Netzwerk in kleinteiligen verbundenen Strukturen und Formaten (Stork, 2014; Smith, Lewis \& Tushman, 2016). Zudem leisten sich resiliente Organisationen an ausgewählten Stellen gezielt Überlappungen/ Doppelarbeiten und sogar redundante Strukturen. Das wirkt auf den ersten Blick zwar ineffizient und unklug. Redundante Strukturen erlauben es den Organisationen aber, auf wechselnde äußere Faktoren und Schocks schneller zu reagieren und ohne große Vorbereitung »die Dinge « ganz anders anzugehen. Man spricht von »shifting modes of decision and management

Wenn wir das »Binnenverhältnis« dieser hybriden Organisationen betrachten, so reichen die Kommunikation und die Zusammenarbeit an den unterschiedlichen Schnittstellen weit über den reinen Informationsaustausch hinaus. Man »denkt gemeinsam«, lässt Widersprüche und unterschiedliche Perspektiven systematisch zu, legt viel Wert auf persönliche Beziehungen und Wertschätzung und ist so in der Lage, systematisch innovative Lösungen für die gesamte Organisation zu entwickeln. In der Summe verfügen diese Organisationen damit auch über eine Agilität, wie sie in anderen Organisationen nicht anzutreffen ist.

\section{Das Konzept der Coaching-Kultur}

"Gemeinsam denken« bedeutet für viele Organisationen, die eher auf klar definierter Informationsweitergabe und hierarchischen Entscheidungsstrukturen aufbauen, einen Paradigmenwechsel auf den Ebenen Zusammenarbeit und interne Kommunikation. Um den Wechsel einzuleiten, kann es für Organisationen hilfreich sein, über den Tellerrand hinauszublicken und sich an der Professionalität des Coachings zu orientieren.

Ein Kollege von uns hatte einen französischen TV-Sender beraten, nachdem dieser von einer kleinen Firma bei der Entwicklung einer Applikation ausgestochen worden war. Die Applikation der kleineren Firma wies nicht nur eine höhere Benutzerfreundlichkeit und mehr Funktionen auf, sie wurde auch 
schneller fertig, und das mit einem Bruchteil an Mitarbeitenden. Unser Kollege beschrieb folgenden Unterschied: »Im großen Unternehmen wurden speziell für dieses Projekt Strukturen und Prozesse geschaffen, um Arbeitspakete zu definieren, Zusammenarbeit effektiv zu steuern und Informationen zeitnah auszutauschen. Das kleine Unternehmen kreierte `Räume « - virtuell und real -, in denen man zusammen denken konnte."

Eine Coaching-Kultur beinhaltet mehr als Akzeptanz für und aktive Förderung von Coaching innerhalb einer Organisation. Coaching-Kultur schafft systematisch Gelegenheiten und »Denkräume« genau dort, wo Menschen gemeinsam an Herausforderungen arbeiten. Dafür braucht es Methoden, Techniken und Haltungen, die vom professionellen Coaching inspiriert sind.

Das Konzept der Coaching-Kultur ergänzt die klassischen Vorstellungen von Organisation und Unternehmenskultur. Die Ergänzung geschieht im Kern dadurch, dass der zunehmenden Komplexität in der neuen Arbeitswelt - und den damit verbundenen Unsicherheiten und Risiken - eine neue Qualität in der persönlichen Beziehung zwischen Führungskraft und Mitarbeitenden und innerhalb von Teams entgegengesetzt wird. Diese Qualität besteht darin, dass man sich über Hierarchien hinweg auf "Augenhöhe« begegnet und gemeinsam über Lösungsmöglichkeiten reflektiert.

Der Weg hin zu einer Coaching-Kultur ist oftmals gleichzusetzen mit einem »Cultural Change Process«. Kommunikation und Zusammenarbeit an den unterschiedlichen Schnittstellen der Organisation entwickeln sich dabei hin zu einem gemeinsamen Reflektieren und einem systematischen Ko-Kreieren von innovativen Lösungen. Dies geschieht nicht in speziellen Workshops oder durch Coaching-Gespräche hinter geschlossenen Türen, sondern es ist eingebettet in den alltäglichen Arbeitsprozess. In diesem Zusammenhang kann man Coaching-Kultur als wirksame Antwort auf die organisationalen Herausforderungen im Zuge der Digitalisierung der Arbeitswelt verstehen.

In den letzten vierzig bis fünfzig Jahren gab es in Organisationen verschiedene Veränderungen und Herausforderungen, bei denen Coaching eine Antwort anzubieten hatte.

- Einzelcoaching durch externe Coaches: Bis in die frühen 1980er Jahren herrschte in Organisationen die Vorstellung vor, dass Lernen in den ersten 25 Lebensjahren abgeschlossen sei. Mitarbeitende konnten davon ausgehen, dass sie nach ihrer Berufsausbildung den Rest ihres beruflichen Lebens eine ähnliche Tätigkeit ausüben würden. Als sich die Arbeitsbedingungen und Anforderungen an Führungskräfte immer schneller wandelten, wurde Einzelcoaching eingeführt, um die Weiterentwicklung im Erwachsenenalter bei diesen neuen beruflichen Herausforderungen zu unterstützen. 
- Führungskraft als Coach: Bis zur Jahrtausendwende wurden alle Fragen der Personalentwicklung in die Kompetenz der HR-Bereiche verlagert. Dort wuchs mit der Zeit die Erkenntnis, dass die Führungskraft eigentlich der erste Personalentwickler sein müsse. Mit dem Konzept »Führungskraft als Coach« fand man eine passende Antwort aus der Welt des Coachings.

- Teamcoaching: Seit gut zehn Jahren werden in Organisationen viele komplexe Herausforderungen in Teams bewältigt. Um Teams in ihrer Performance zu unterstützen, hat sich der Ansatz des Teamcoachings etabliert.

- Coaching-Kultur: Mit dem Bedarf nach agilen und resilienten Organisationen steht eine neue Herausforderung an. Auch da kann Coaching einen Beitrag leisten, wenn sich eine Coaching-Haltung und ein Coaching-Vorgehen an den Kommunikations- und Zusammenarbeits-Schnittstellen einer Organisation durchsetzt. In dem Moment sprechen wir von Coaching-Kultur (vgl. Abbildungen 1 und 2).

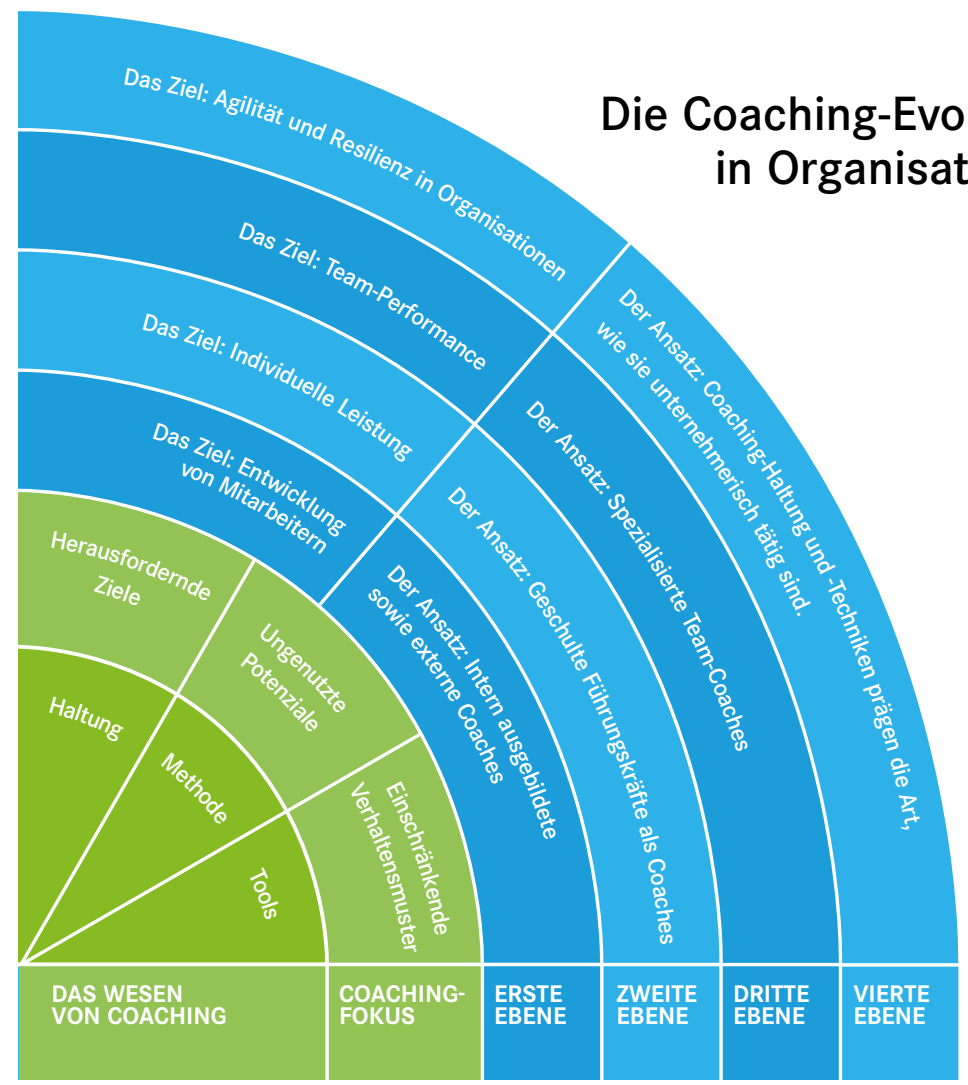

Abbildung 1: Antworten von Coaching auf Organisationsherausforderungen 
Coaching-Kultur ist keine eindeutige Größe, sondern setzt sich aus verschiedenen, miteinander verwobenen Komponenten zusammen (vgl. Abbildung 2).

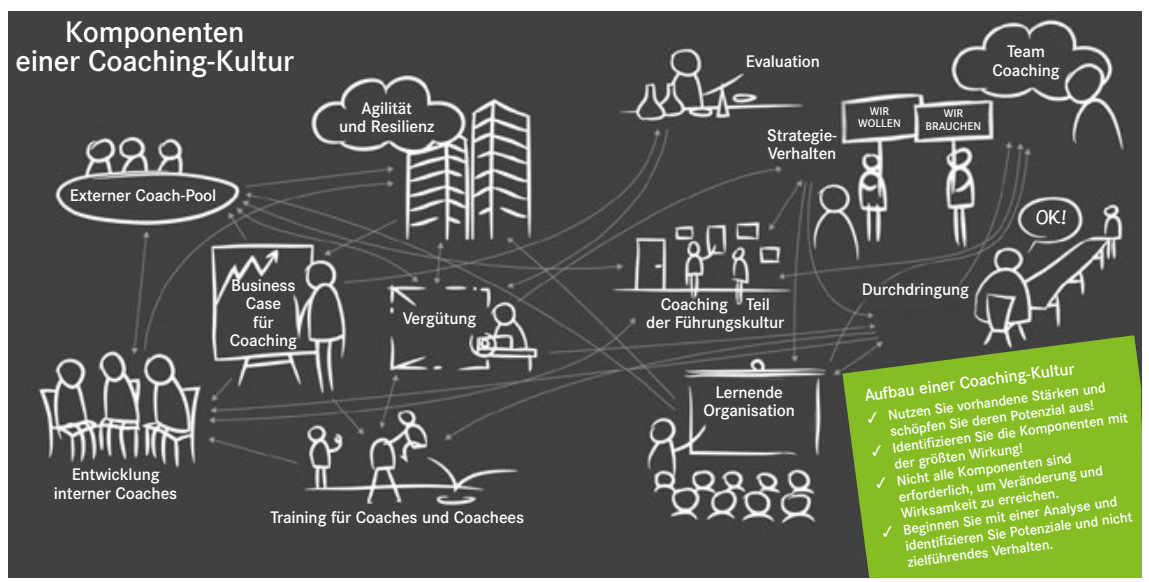

Abbildung 2: Systemische Wechselwirkungen einer Coaching-Kultur

In »Parachuting Cats into Borneo« (Klimek \& AtKisson, 2016) beschreiben Axel Klimek und Alan AtKisson die vielfältigen, subtilen systemischen Wirkmechanismen, die einen Cultural-Change-Prozess erfolgreich werden lässt. Die Entwicklung einer Coaching-Kultur entspricht einem solchen CulturalChange-Prozess, Kernelemente und Qualitäten aus dem personenbezogenen, »klassischen « Coaching-Verständnis werden auf die vielfältigen Beziehungen zwischen den organisatorischen Elementen (Einheiten, Gruppen, Teams, Abteilungen usw.) innerhalb einer Organisation übertragen. Die Beziehungsqualität des personenbezogenen klassischen Coachings wird damit auf subtile Art und Weise zu einem zentralen Faktor der Unternehmenskultur. Es geht beim Aufbau einer Coaching-Kultur weniger darum, alle Komponenten (vgl. Abbildung 2) einzuführen, sondern darum, herauszufinden, welche Faktoren und systemischen Wechselwirkungen die größte positive Wirkung im Hinblick auf eine organisationsweite Durchdringung von Coaching-Haltung und Coaching-Verständnis haben könnten.

Um Organisationen zu unterstützen, ihren spezifischen Ansatzpunkt zur Einführung einer Coaching-Kultur zu identifizieren, hat das Center for Sustainability Transformation einen organisationsdiagnostischen Fragebogen entwickelt. Gemeinsam mit der Hochschule Darmstadt wurde auf Basis dieses Ansatzes eine Vergleichsstudie in deutschsprachigen Organisationen durchgeführt, bei der unter anderem der Ausprägungsgrad der Integration einer Coaching-Kultur erhoben wurde. 


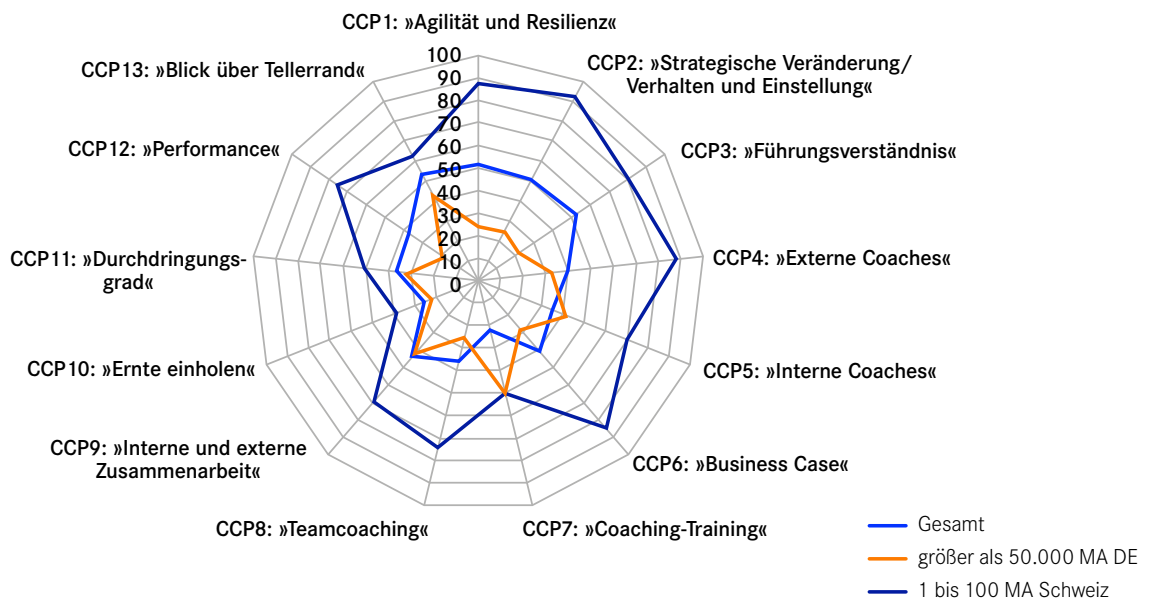

Abbildung 3: Vergleichsauswertung für eine Organisation aus Deutschland mit über 50000 Mitarbeitenden

Die Grafik zeigt eine Organisation aus Deutschland mit über 50000 Mitarbeitenden im Vergleich mit der »besten Organisation« aus der Studie und dem Durchschnitt (»Gesamt«) aller daran beteiligen Organisationen. Die beste Organisation stammte aus der Kategorie »bis hundert Mitarbeitende« (schwarze Linie, außen). Die Vergleichsgrafik zeigt deutlich, dass das beauftragende Unternehmen (graue Linie, mehrheitlich im Innern des Netzes) bei den meisten Komponenten schlechter abschneidet als der Durchschnitt, in einigen gleich gut ist und nur in den Bereichen »Coaching-Training « und »Interne Coaches" über dem Durchschnitt liegt. Bei der Komponente »Coaching-Training« ist das Unternehmen genauso stark wie die beste Organisation. In allen anderen Bereichen gibt es einen markanten Unterschied. Für das Unternehmen waren die Ergebnisse sowohl die Bestätigung, dass sie in den Bereichen, die ihm in den letzten Jahren wichtig waren, beim Aufbau eines Pools von internen Coaches und bei den Trainings für sie, auf dem richtigen Weg ist. Gleichzeitig sind ihm verschiedene Handlungsfelder gespiegelt worden, in denen es aktiv werden kann. Als ein erster Schritt wurde »Ernte einholen« identifiziert. Man erhofft sich durch die Rückspiegelung der Erfahrungen und Erkenntnisse aller im Unternehmen tätigen Coaches Rückschlüsse und präzise Ideen für die Themen »Führungsverständnis«, »Performance«, »Agilität und Resilienz« sowie »Verhalten und Einstellung«. 


\section{Coaching-Kultur in deutschsprachigen Organisationen - die Studie}

Oben haben wir den Nutzen des Fragebogens für ein einzelnes Unternehmen beschrieben. Für die Studie zur »Coaching-Kultur in deutschsprachigen Organisationen " wurden bisher siebzig Unternehmen aus unterschiedlichen Branchen in Deutschland, Österreich und der Schweiz befragt, und zwar zu verschiedenen Dimensionen und Themenstellungen:

- Business Case für Coaching,

- Agilität und Resilienz,

- Coaching im Führungsverständnis,

- Konzept für den Einsatz externer Coaches,

- Einsatz von internen Coaches oder Mentoren,

- Konzept für Teamcoaching,

- Coaching in der täglichen Arbeit und zur Performancesteigerung,

- organisationsweites Lernen aus den Coaching-Maßnahmen und Grad der Durchdringung der Organisation mit dem Coaching-Ansatz,

- lernende Organisation - Blick nach außen,

- Durchdringungsgrad von Coaching innerhalb der Organisation,

- Coaching bei strategischen Veränderungen,

- Training für Coaches und Coachees,

- Coaching an den Schnittstellen der Zusammenarbeit - intern und extern.

Zwei Fragestellungen haben uns bei der Studie geleitet:

- Wie stark sind die verschiedenen Dimensionen in Organisationen unterschiedlicher Größe ausgeprägt?

- Gibt es eine Korrelation mit unternehmensrelevanten Themen wie Integration von Nachhaltigkeit/CSR, finanziellem Erfolg, Innovationskraft, Attraktivität als Arbeitgeber oder Mitarbeitendenzufriedenheit?

\section{Stichprobe und Methodik}

An der Studie zur »Coaching-Kultur in deutschsprachigen Organisationen «, die aus einem Onlinefragebogen mit 89 Fragen besteht, haben im Zeitraum Mai 2015 bis Februar 201670 Organisationen aus unterschiedlichen Branchen in Deutschland, Österreich und der Schweiz teilgenommen.

\section{Ergebnisse}

Die Ergebnisse der Studie deuten darauf hin, dass eine ausgeprägte Coaching-Kultur mit verschiedenen unternehmensrelevanten Kriterien positiv kor- 
reliert. Studienteilnehmerinnen und Studienteilnehmer, die ihr Unternehmen als finanziell erfolgreicher und innovativer als die Konkurrenten einschätzen, haben in ihrem Unternehmen eine stärker ausgeprägte Coaching-Kultur (44 \%) identifiziert. Teilnehmende, die ihr Unternehmen als finanziell weniger erfolgreich und innovativ als die Konkurrenz einschätzen, haben in ihrem Unternehmen eine schwächer ausgeprägte Coaching-Kultur (30 \%) wahrgenommen. Bei den Parametern Mitarbeitendenzufriedenheit ( $47 \%$ zu $34 \%$ ) und Arbeitgeberattraktivität ( $45 \%$ zu $22 \%$ ) gab es ebenfalls signifikante Unterschiede. In Organisationen mit hoher Mitarbeitendenzufriedenheit ist die Coaching-Kultur um 13 Prozent stärker ausgeprägt und bei hoher Arbeitgeberattraktivität sogar um 23 Prozent.

Interessant ist auch, dass kleine Unternehmen mit bis zu 100 und große Unternehmen mit über 50000 Angestellten aus Sicht der Mitarbeitenden eine höher ausgeprägte Coaching-Kultur aufweisen als das weite Feld der Organisationen zwischen 100 und 50000 Mitarbeitenden.

Organisationen, die in den Themen Corporate Social Responsibility und Nachhaltigkeit stark verankert wahrgenommen werden, haben im Vergleich mit allen anderen abgefragten Indikatoren die höchste Ausprägung (57 \%) einer Coaching-Kultur. In den Organisationen, in denen Corporate Social Responsibility und Nachhaltigkeit als nicht sehr stark verankert erlebt werden, sinkt der Wert der Coaching-Kultur auf 29 Prozent.

\section{Fazit}

Fachleute sprechen vielfach von systematischen Ressourcendefiziten und einer regelrechten Ressourcenkrise angesichts der Belastungen, denen Menschen und Organisationen durch die laufenden Anpassungs- und Veränderungsprozesse ausgesetzt sind. Insbesondere die kognitiven Fähigkeiten und die psychisch-mentalen Ressourcen werden immer stärker beansprucht - als Folge des Anstiegs und der Intensivierung der Informations- und Wissensarbeit und der steigenden Komplexitäten, Abhängigkeiten und Unsicherheiten in der Arbeit. Faktoren wie Resilienz und Reagibilität haben eine wesentliche Bedeutung für die Innovationsfähigkeit, Kreativität, Konflikt- und Kommunikationsbereitschaft und damit für den wirtschaftlichen Erfolg von Organisationen. Die Hochschule Darmstadt will hier über den Ansatz der Resilienten Organisation der Digitalen Arbeit (RODA) einen Beitrag zum besseren Management- und Organisationsverständnis in der wissenschaftlichen Forschung leisten und für die betriebliche Praxis hilfreiche Konzepte und Impulse für eine nachhaltige Organisationsentwicklung in der neuen Arbeitswelt bieten. Das vom Center for Sustainability 
Transformation entwickelte Konzept der Coaching-Kultur bildet dabei einen wichtigen Baustein des RODA-Ansatzes.

Die gemeinsame Studie zur »Coaching-Kultur in deutschsprachigen Organisationen « des Center for Sustainability Transformation und der Hochschule Darmstadt erlaubt wirksame Vergleiche zur Coaching-Kultur von Organisationen in den verschiedenen Branchen und in Unternehmen unterschiedlicher Größenordnung. Nicht nur scheint sich eine Coaching-Kultur positiv auf die Zufriedenheit der Mitarbeitenden in der Organisation auszuwirken, sondern sie stärkt offenbar auch die Innovationskraft des Unternehmens und erhöht seinen finanziellen Erfolg. Eine mögliche Erklärung könnte darin liegen, dass durch Coaching an den alltäglichen Schnittstellen einer Organisation die Mitarbeitenden sich als gleichwertige Gesprächspartner angesehen fühlen, die bei wichtigen Herausforderungen auf Augenhöhe einbezogen werden. Dies kann auf der einen Seite zu einer Stärkung der Innovationskraft in der Organisation führen und anderseits über die höhere Einbindung auch zu einer gesteigerten Zufriedenheit beitragen. Beides kann mittelfristig zu einer effizienteren Nutzung der Ressource »Mensch« beitragen und sich positiv auf finanzielle Kennzahlen auswirken - direkt über eine Produktivitätssteigerung oder indirekt über einer Senkung der Fehlzeiten durch Krankheit und eine Erhöhung des Engagements und des Commitment.

Bei diesen Aspekten sollten künftige Studien und Forschungen aus unserer Sicht ansetzen und den in diesem Erklärungsansatz formulierten Wirkmechanismen nachgehen.

\section{Literatur}

Birk, S., Nelsen, M., Lackerbauer, S., Kirchner, C. (2015). Wie wir morgen arbeiten ... Studie auf Basis einer Stichprobe von 171 Quellen zur Arbeit der Zukunft. München: Institut für Arbeitsdesign und Zukunftstechnologien e. V. www.i-faz.de/ecosystem/studie [11.3.2017].

Klimek, A., AtKisson, A. (2016). Parachuting Cats into Borneo - And Other Lessons from the Change Café. White River Junction, VT: Chelsea Green Publishing.

Lohmann-Haislah, A. (2012). Stressreport Deutschland 2012, Psychische Anforderungen, Ressourcen und Befinden. Berlin: Bundesanstalt für Arbeitsschutz und Arbeitsmedizin. www.baua.de/de/ Publikationen/Fachbeitraege/Gd68.pdf?__blob=publicationFile [11.3.2017].

Rose, U., Müller G., Burr, H., Schulz, A., Freude, G. (2016). Arbeit und Mentale Gesundheit - Ergebnisse aus einer Repräsentativerhebung der Erwerbstätigen in Deutschland. Berlin: Bundesanstalt für Arbeitsschutz und Arbeitsmedizin. www.baua.de/de/Publikationen/Fachbeitraege/ F2250-2.pdf?_blob=publicationFile\&v=5 [11.3.2017].

Smith, W. K., Lewis, M. W., Tushman, M. L. (2016). Sowohl-als-auch-Management. Harvard Business manager, (August).

Stork, W. (2014). Die wirtschaftliche Bedeutung von Stress. In C. Haurand, H. Ullrich, M. Weniger (Hrsg.), Stressmedizin: Beratung, Vorbeugung, Behandlung. Berlin: Medizinisch Wissenschaftliche Verlagsgesellschaft.

Weinreich, I., Weigl, C. (2011). Unternehmensratgeber betriebliches Gesundheitsmanagement. Grundlagen - Methoden - personelle Kompetenzen. Berlin: Erich Schmidt. 


\section{Die Autorinnen und Autoren, Herausgeberinnen und Herausgeber}

Marion Alt, Betriebsökonomin, systemisch-lösungsorientierte Coach und Mediatorin; langjährige Erfahrung in der Beratung und Begleitung von komplexen Veränderungsprozessen in unterschiedlichen Branchen und Bereichen in der Schweiz und in Deutschland; Spezialistin in Mediation, Verhandlungsführung, Coaching und Teamentwicklung (u. a. tätig in den Bereichen Pharma, Maschinenbau, Krankenkassen, Automotive, Hochschulen wie FHNW, HSLU, Berufsbildung u.a.m.).

E-Mail: marion.alt@fhnw.ch

Elke Berninger-Schäfer, Dr., Inhaberin des Karlsruher Instituts für Coaching, geschäftsführende Gesellschafterin der CAI GmbH, Diplom-Psychologin, Senior Coach DBVC, Lehrcoach, Supervisorin BDP, approbierte Psychotherapeutin; Anbieterin von DBVC-anerkannte Coaching-Weiterbildungen und eines Coachpools.

E-Mail: info@berninger-schaefer.de

Isabell Braumandl, Dipl.-Psych., Dipl.-Ök., Inhaberin des Coaching- \& BeratungsCentrums Regensburg, Lehrbeauftragte/Dozentin an den Universitäten Salzburg und Braunschweig und an der Munich Business School.

E-Mail: Info@cobece.de

Gery Bruederlin, Prof. Dr., Professor und Dozent für Human Resource Management an der FHNW in Olten und Basel, Leiter des MAS Human Resource Management; anerkannter Experte für die Entwicklung und Implementierung von modernen HR-Konzepten und HR-Dienstleistungsangeboten.

E-Mail: gery.bruederlin@fhnw.ch 
Silvia Deplazes, Prof. Dr., MBA, Co-Leiterin der Fachgruppe Diagnostik und Beratung, Leitung angewandte psychologische Methoden, Lehre und Forschung im Bereich Beratung/Coaching am Departement Angewandte Psychologie der Zürcher Hochschule für Angewandte Wissenschaften, systemische Organisationsberaterin.

E-Mail: deze@zhaw.ch

Wolfgang J. Eberling, Prof. Dr., Professor für Organizational Behavior an der FHNW in Olten und Basel; hat dort Coaching-Weiterbildungen entwickelt, leitet den CAS Change Management und ist als Consultant und Coach für Executives, Teams und Change-Verantwortliche in Firmen, NGOs und Verwaltungen tätig. E-Mail: wolfgang.eberling@fhnw.ch

Harald Geißler, Prof. Dr., Studium der Erziehungswissenschaft, Promotion 1976, Habilitation 1985; im selben Jahr für das Fach Erziehungswissenschaft, insbesondere Berufs- und Betriebspädagogik, an die Helmut-Schmidt-Universität Hamburg berufen; leitet dort am Management Development Center das Competence Center Coaching mit den beiden Schwerpunkten Coaching-Gutachten und Virtuelles Coaching; betreute im Zusammenhang mit seinen Forschungsschwerpunkten Organisationslernen und Coaching eine Vielzahl an Projekten der Führungskräfte- und Organisationsentwicklung.

E-Mail: Harald.Geissler@hsu-hh.de

Laura Glauser, Dr., Ethnologin, arbeitet an der Universität Hamburg im Bereich Personalentwicklung; seit 2007 Tätigkeit als systemische Beraterin/Coach mit dem Schwerpunkt Hochschulen; Dissertation über das »unternehmerische Selbst« im Kontext von Career Services; lehrt v. a. zu den Themen Arbeits- und Organisationsethnologie.

E-Mail: kontakt@laura-glauser.com

Marianne Hänseler, Dr. phil., Dozentin und stv. Studienleitung des Master of Advanced Studies in Coaching an der Hochschule für Soziale Arbeit, Fachhochschule Nordwestschweiz. Zudem freiberufliche Coach, Supervisorin (BSO) und Philosophin.

E-Mail: marianne.haenseler@fhnw.ch 
R. Niki Harramach, Prof. Dr., Harramach \& Veličković, geschäftsführender Gesellschafter, ehem. Panzerkommandant, emeritierter Rechtsanwalt, akkreditierter Wirtschaftstrainer und Wirtschaftscoach, Supervisor, Sachverständiger für Managementtrainings und OE. OE-Lehrtrainer. Spezialthemen: Soft Skills, interaktive Verhaltenstrainings, Diversity Management, Rechtsfragen und Erfolgskontrolle im Bildungsbereich. Verfasser diverser Fachartikel und Bücher. E-Mail: office@harramach.com

www.harramach.com

Mathias Hofmann, Dipl.-Päd., Master of Business Consulting; geschäftsführender Gesellschafter der SHS CONSULT GmbH in Bielefeld seit 2002 (www. shs-consult.de); Schwerpunkte: Beratung und Coaching im Change Management, Moderation und Steuerung von Veränderungsprozessen, Ausbildung und Coaching von Führungskräften; zertifizierter Coach (EASC) und Gestaltberater (FPI); Mitglied im Vorstand der European Association for Supervision and Coaching (EASC e. V.); Lehrbeauftragter der FH Mittelstand in Bielefeld zu Führung, Selbstmanagement und Arbeits- und Organisationspsychologie; Veröffentlichungen u. a.: »Fit for Change« $(2007,2009)$, »Change Controlling « (2011), »Stress-Kompass « (2015).

E-Mail: mh@shs-consult.de

Willem Jan Hofmans, DBA; has been a Leadership Coach since 2007; before this spent 25 years as a senior executive in the Healthcare and IT sector; in addition to his Doctorate of Business Administration from Grenoble École de Management (2015), he is a Professional Certified Coach by the ICF (2012); his research interest is the effectiveness of coaching.

E-Mail: willem.jan.hofmans@hfsc.ch

Eva Jonas, Univ.-Prof. Dr., Leiterin des Fachbereichs Psychologie und der Abteilung Sozialpsychologie an der Universität Salzburg.

E-Mail: Eva.Jonas@sbg.ac.at

Efriom Kineselassie, Mitarbeiter des Karlsruher Instituts für Coaching, geprüfter Wirtschaftsfachwirt (IHK); studiert Wirtschaftspsychologie in Mannheim und absolviert neben dem Studium eine Weiterbildung zum Business-Coach und Gesundheits-Coach.

E-Mail: Efriom.Kineselassie@cai-world.com 
Axel Klimek, Prof. Dr., Geschäftsführer des CforST - Center for Sustainability Transformation, $\mathrm{GmbH}$ und Lehrbeauftragter an der Hochschule Darmstadt (h_da); seit über 30 Jahren als Coach tätig, seit 17 Jahren zusätzlich als Organisationsentwickler und -berater; war in über 30 Ländern tätig; zu seinen Kunden gehören unter anderem Allianz, EY, GIZ, Lufthansa, T-Systems und Unilever. Axel Klimek ist Leiter der Studie »Coaching Kultur in deutschsprachigen Organisationen «.

E-Mail: axel.klimek@CforST.com

Axel Koch, Prof. Dr., Dipl.-Psych., Professor für Training und Coaching an der Hochschule für angewandtes Management in Erding. Gehört zu den führenden Experten für nachhaltig wirksame Personalentwicklung; hat durch seinen unter dem Pseudonym Richard Gris verfassten Wirtschaftsbestseller »Die Weiterbildungslüge« (2008, Campus) breite Bekanntheit erlangt; die Zeitschrift»OrganisationsEntwicklung« zählt ihn deshalb zu den »wichtigsten Vordenkern zu den wesentlichen Fragen zur Zukunft von HR« (Dossier 2/12); seine Transferstärke-Methode ${ }^{\circledast}$ gehörte zu den Preisträgern beim Deutschen Weiterbildungspreis 2011.

E-Mail: axel.koch@fham.de

Hansjörg Künzli, Prof., Co-Leiter der Fachgruppe Diagnostik und Beratung, Leitung Forschungsmethoden, Lehre und Forschung in den Bereichen Beratung, Diagnostik und Methodenlehre am Departement Angewandte Psychologie der Zürcher Hochschule für Angewandte Wissenschaften.

E-Mail: kasg@zhaw.ch

Sabine Losch, B. Sc., M. Sc., Psychologin. Seit 2011 zertifizierte Coach und Co-Trainerin im Rahmen der Ausbildung für Karriere- und Lebensplanungscoaching in Zusammenarbeit mit dem Coaching- \& Beratungs-Centrum Regensburg (CoBeCe). Seit 2014 wissenschaftliche Mitarbeiterin und Doktorandin in der Abteilung für Wirtschafts- und Organisationspsychologie an der Universität Salzburg. Forschung zur Wirkung und Wirksamkeit von Coachingund Trainingsmaßnahmen sowie Coach-Klienten-Interaktion.

E-Mail: sabine.losch@sbg.ac.at 
Jasmin Messerschmidt, Dr., Dipl.-Psych., Senior Coach DBVC; 2003 Gründung von Messerschmidt Coaching; seitdem selbstständig tätig als Coach und Beraterin für Führungskräfte und Teams in Unternehmen; Schwerpunkte: Persönlichkeitsentwicklung; Vertrauenskulturen in Teams; 2014 Promotion mit der Arbeit "Das Selbst im Bild. Eine empirische Studie zum Einsatz von Bildmaterialien zur Förderung von Selbstreflexions- und Selbstveränderungsprozessen im Einzelcoaching «, erschienen im Verlag Peter Lang.

E-Mail:jm@messerschmidt-coaching.de

Christina Mühlberger, Mag. Dr., Mitarbeiterin der Abteilung Sozialpsychologie an der Universität Salzburg, zertifizierte Karriere-Coach.

E-Mail: Christina.Muehlberger@sbg.ac.at

Maximilian David Mühlberger, Mag. Dr., Studium der Psychologie/BA in Pädagogik; seit 2012 Doktorand am Fachbereich Psychologie, Abteilung Wirtschafts- und Organisationspsychologie, der Universität Salzburg; Doktorarbeit zum Thema "Leader of the coaching process: The transformational and transactional coach " (2016); seit 2012 Mitbetreuung der Coaching-Ausbildung an der Universität Salzburg in Kooperation mit dem CoBeCe Regensburg (Isabell Braumandl).

E-Mail: maximilian.muehlberger@sbg.ac.at

Stefanie Neumann, MSc., Beraterin und Dozentin für Leadership und Coaching am IAP Institut für Angewandte Psychologie der Zürcher Hochschule für Angewandte Wissenschaften. Leitung des CAS Coaching Advanced und CAS International Leader \& Entrepreneur. Schwerpunkte in den Themen Kommunikation, Coaching, Teamentwicklung, Selbstmanagement, Begleitung von Veränderungsprozessen, interkulturelle Kompetenz.

E-Mail: stefanie.neumann@zhaw.ch

Alica Ryba, Dipl.-Kauffrau, promoviert bei Prof. Gerhard Roth zum Thema Coaching. Inhaberin von ARYBA Coaching in Hamburg, publiziert Fachbücher und hat eine integrative (Rauen \& Steinhübel), eine systemische (ISB Wiesloch), eine hypnotherapeutische (MEI Hamburg) und eine generative (Gilligan \& Dilts) Coaching-Ausbildung absolviert.

E-Mail: dialog@aryba.de 
Annamarie Ryter, Dr. Prof. FH., Dozentin Berufspraktische Studien der Pädagogischen Hochschule FHNW, Dozentin im CAS »Von der Schule zum Beruf«. Mitinhaberin der Firma bildbar, Basel.

E-Mail: annamarie.ryter@fhnw.ch

Sandra J. Schiemann, M.Sc., B. Sc., Doktorandin der Sozialpsychologie, Universität Salzburg; zertifizierte Karriere-Coach, Trainerin, Mentorin.

E-Mail: Sandra.Schiemann@sbg.ac.at

Werner Stork, Prof. Dr., Professor für Organisation und Management, insbesondere Personalmanagement und Führung, an der Hochschule Darmstadt (h_da); leitet dort das Forschungs-/Projektprogramm RODA - Resiliente Organisation der Digitalen Arbeit und ist Mitorganisator des Wirtschaftsforums der h_da; war nach seiner Promotion am Institut für Genossenschaftswesen der WWU Münster 13 Jahre lang in verschiedenen Unternehmen in leitenden oder geschäftsführenden Funktionen tätig; arbeitet als Coach und berät Unternehmen zur Organisationsentwicklung - mit dem Schwerpunkt auf Corporate Learning und Corporate Health.

E-Mail: werner.stork@h-da.de

Cornelia Tonhäuser, Dr., Dipl.-Päd., seit 2012 Juniorprofessorin für Wirtschaftspädagogik mit dem Schwerpunkt Bildungsmanagement an der Wirtschaftswissenschaftlichen Fakultät der Universität Göttingen; Forschungsschwerpunkte: betriebliches Bildungsmanagement, Wirksamkeit und Lerntransfers beruflicher Weiterbildung, Gestaltung von Lern-, Entwicklungs- und Qualifizierungsprozessen in der beruflichen Bildung; ihre 2010 veröffentliche Dissertation untersucht die »Implementierung von Coaching als Instrument der Personalentwicklung in deutschen Großunternehmen «.

E-Mail: cornelia.tonhaeuser@wiwi.uni-goettingen.de

Eva Traut-Mattausch, Dr., Univ.-Prof., Dipl.-Psych., Betriebswirtin (VWA), Leiterin der Abteilung Wirtschafts- und Organisationspsychologie, des Universitätslehrgangs für Supervision, Coaching und Mediation sowie des Doktoratskollegs »Bestehen in modernen Arbeitswelten: Leben und Arbeiten 4.0 $(\operatorname{BimA})$ « an der Universität Salzburg; stellvertretende Leiterin des Fachbereichs Psychologie und Vizedekanin der dortigen Naturwissenschaftlichen Fakultät. E-Mail: eva.traut-mattausch@sbg.ac.at 
Nina Veličković, Mag., Harramach \& Veličković, geschaftsführende Gesellschafterin, Psychotherapeutin, Unternehmensberaterin, gewerbliche Wirtschaftstrainerin, systemische Coach und akkredierte Wirschaftscoach. Lehrtätigkeit Coaching, Schwerpunkte: Persönlichkeitsentwicklung, Teamentwicklung/ Rangdynamik, Management-Inszenierung, Grenzbereich Psychotherapie und Coaching.

E-Mail: office@harramach.com

www.harramach.com

Julia Weber, Dr., Institut für Selbstmanagement und Motivation Zürich (ISMZ), Geschäftsführerin, zertifizierte ZRM-Trainerin und Coach, Ausbildungstrainerin für Zertifikatslehrgänge ZRM-Trainer und Coach, Forscherin, Autorin. E-Mail: julia.weber@ismz.ch

Robert Wegener, Dr., Coach, Dozent, M. A. Politologie und M. A. Kommunikationspsychologie. Co-Leiter der Coaching Studies FHNW (www.coachingstudies.ch). Seit 2010 Leiter der Internationalen Coaching-Kongresse (www. coaching-meets-research.ch). Forschung zu Coachingprozessen (»Bedeutsame Momente im Coaching «). Mitherausgeber u. a. von »Coaching und Gesellschaft» (2016), »Bewertung von Coachingprozessen « (2015) und »Coaching-Praxisfelder« (2014).

E-Mail: robert.wegener@fhnw.ch

Wolfgang Widulle, Dr. rer. soc., Dipl.-Päd. univ., Erwachsenenbildner, Trainer, Autor, Schreiner. Dozent an der Hochschule für Soziale Arbeit FHNW am Institut für Beratung, Coaching und Sozialmanagement. Arbeitsschwerpunkte: Handlungstheorien in Psychologie und Sozialer Arbeit; pädagogische Psychologie und Fachdidaktik in der Sozialen Arbeit; Kommunikation, Gesprächsführung, Beratung, Training; Kasuistik/Hilfeplanung.

E-Mail: wolfgang.widulle@fhnw.ch

Mirjam Zanchetta, Dr., B. Sc., M. Sc., Universität Salzburg, Senior Scientist; Forschungsschwerpunkt: Impostor-Phänomen; zertifizierter Karriere- und Lebensplanungs-Coach.

E-Mail: mirjam.zanchetta@bg.ac.at 